PROGRAMA DE DOCTORADO EN INGENIERÍA INDUSTRIAL

TESIS DOCTORAL:

\title{
INVESTIGACIÓN Y DESARROLLO DE MATERIALES AVANZADOS Y SOSTENIBLES PARA UNA NUEVA APLICACIÓN DE PULTRUSIÓN TERMOPLÁSTICA
}

Presentada por María Asensio Valentín para optar al grado de Doctor/a por la Universidad de Valladolid

Dirigida por:

Dra. Karina Núñez Carrero

Dr. José María Pastor Barajas

Dr. Juan Carlos Merino Senovilla 


\section{ÍNDICE}

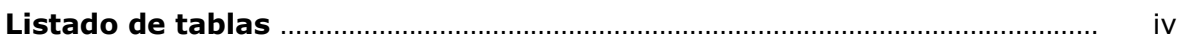

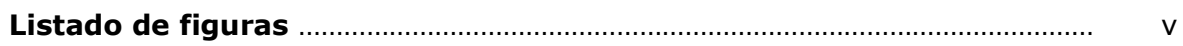

\section{Capítulo -1}

MOTIVACIÓN, OBJETIVOS Y ESTRUCTURA DE LA INVESTIGACIÓN

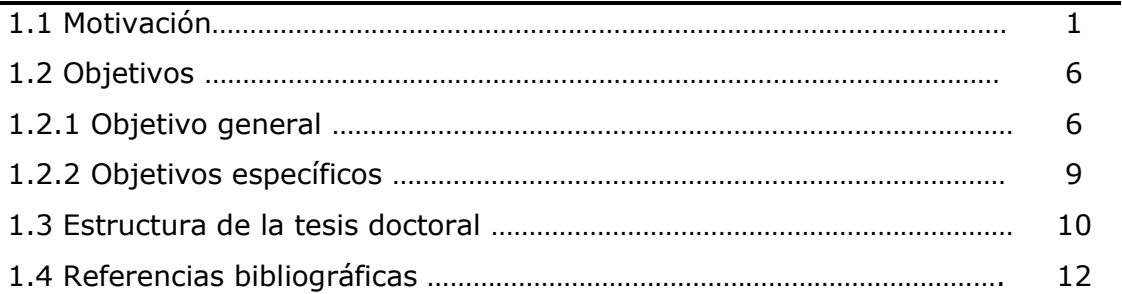

\section{Capítulo -2}

\section{POLIETILEN TEREFTALATO Y MATERIALES COMPUESTOS}

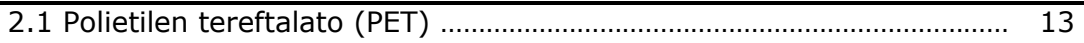

2.1 .1 Historia y aplicaciones .............................................................. 13

2.1 .2 Síntesis .................................................................................. 14

2.1 .3 Reciclado ...................................................................................... 15

2.2 Materiales compuestos ......................................................................... 20

2.2.1 Aspectos generales ....................................................................... 20

2.2.2 Procesos de obtención de composites de fibra continua: 24 pultrusión

2.3 Referencias bibliográficas 


\section{Capítulo -3}

\section{TÉCNICAS EXPERIMENTALES: MODIFICACIÓN REOLÓGICA, METODOLOGÍA} DE PROCESADO Y CARACTERIZACIÓN DE COMPOSITES TERMOPLÁSTICOS

3.1 Técnicas de modificación reológica ....................................................... 36

3.2 Técnicas de caracterización de materiales modificados ....................... 39

3.2.1 Análisis térmico ............................................................................... 39

3.2.2 Comportamiento mecánico .................................................................... 40

3.2.3 Medidas de viscosidad ......................................................................... 42

3.2.4 Métodos espectroscópicos ……………………................................ 46

3.2.5 Otras técnicas instrumentales de caracterización .............................. 47

3.3 Desarrollo de una cámara de impregnación ......................................... 47

3.3.1 Diseño del proceso de impregnación ................................................. 47

3.3.2 Desglose de los componentes del proceso de impregnación ........... 48

3.4 Línea de pultrusión en fundido ............................................................. 52

3.5 Línea de pultrusión en polvo .................................................................... 52

3.5.1 Towpregger (equipo de impregnación en polvo) .............................. 52

3.5.2 Máquina de pultrusión ...................................................................... 55

3.6 Técnicas de caracterización de perfiles ................................................ 57

3.6.1 Comportamiento mecánico …………………............................... 57

3.6.2 Microscopía ........................................................................................ 59

3.6.3 Otras técnicas instrumentales de caracterización .............................. 59

3.7 Referencias bibliográficas ............................................................... 60

\section{Capítulo -4}

MATERIALES, MODIFICACIÓN REOLÓGICA Y CARACTERIZACIÓN

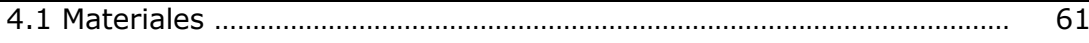

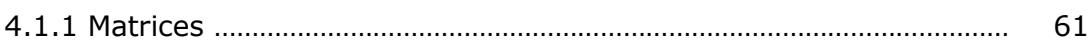

4.1.2 Caracterización de los materiales de PET .......................................... 63

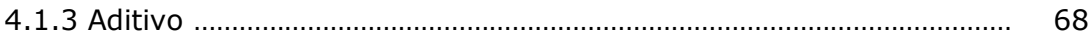

4.2 Modificación reológica de la matriz polimérica …................................ 69

4.2.1 Degradación termo-mecánica del PET virgen ................................. 71

4.2.1.1 Resultados de la degradación termo-mecánica del PET virgen ... 72

4.2.2 Degradación hidrolítica del PET virgen .............................................. 80

4.2.2.1 Resultados de la degradación hidrolítica del PET virgen ............... 81

4.2.3 Modificación química del PET virgen ............................................... 85

4.2.3.1 Resultados de la modificación química del PET virgen .................. 86

4.2.4 Modificación física y modificación química de las mezclas .............. 93

4.2.4.1 Resultados de la modificación física y modificación química de 95 las mezclas 


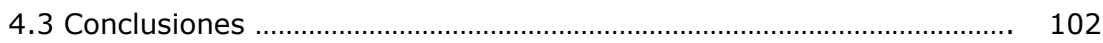

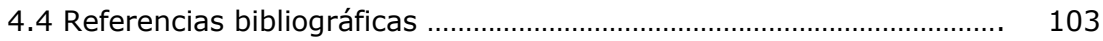

\section{Capítulo -5}

\section{OBTENCIÓN Y CARACTERIZACIÓN DE PERFILES}

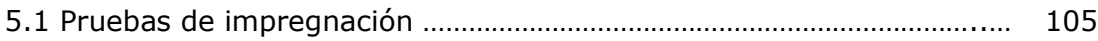

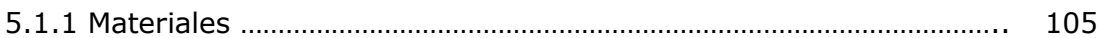

5.1.2 Obtención de tapes (pre-impregnado) ......................................... 106

5.1.3 Caracterización de los tapes ................................................... 108

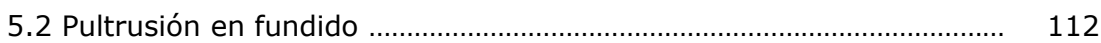

5.2 .1 Obtención de perfiles .............................................................. 112

5.2 .2 Caracterización de perfiles ....................................................... 113

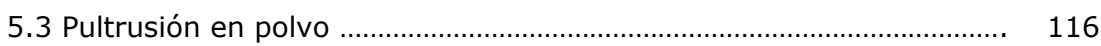

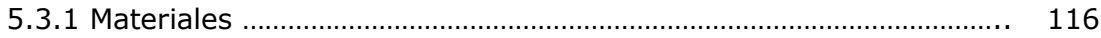

5.3.2 Obtención de towpreg (pre-impregnado) …................................... 118

5.3.3 Obtención de perfiles ..................................................................... 120

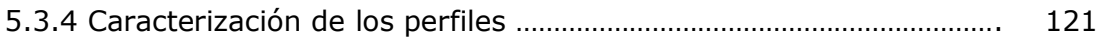

5.4 Comparación con matrices termoestables convencionales ................... 126

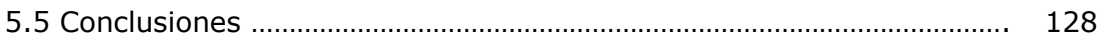

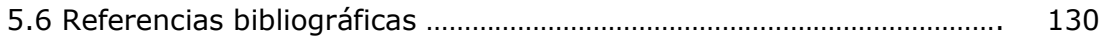

\section{Capítulo -6}

\section{CONCLUSIONES Y PERSPECTIVAS FUTURAS}

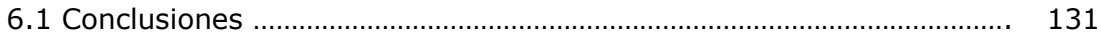

6.1.1 Conclusiones del trabajo de investigación .................................... 131

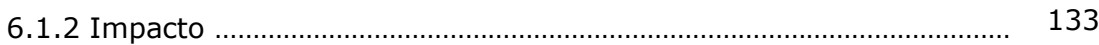

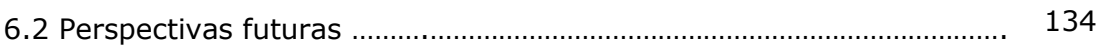

\section{Anexos}

Contribuciones en congresos

Publicaciones 


\section{LISTADO DE TABLAS}

\section{Capítulo 2}

Tabla 2.1 Propiedades mínimas de escamas procedentes de rPET para su procesado

\section{Capítulo 3}

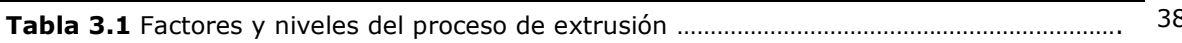

Tabla 3.2 Dimensiones de la probeta de ensayo de tracción ....................................................... 58

Tabla 3.3 Dimensiones de la probeta de ensayo de flexión .................................................... 58

\section{Capítulo 4}

Tabla 4.1 Descripción de los materiales de PET utilizados 61

Tabla 4.2 Propiedades térmicas de los materiales PET de partida ............................................ 65

Tabla 4.3 Propiedades mecánicas de los materiales utilizados .................................................. 68

Tabla 4.4 Factores y niveles del proceso de extrusión .......................................................... 71

Tabla 4.5 Matriz de experimentos para la optimización de las condiciones de extrusión ....... 72

Tabla 4.6 Resultados de índice de fluidez, viscosidad intrínseca y peso molecular 76

viscosimétrico de las muestras del vPET modificadas termo-mecánicamente .......................... Tabla 4.7 Propiedades térmicas de las muestras de vPET modificado termo- 77

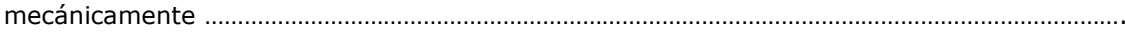

Tabla 4.8 Módulo de Young de las muestras de vPET modificado termo-mecánicamente ..... 79

Tabla 4.9 Composición y condiciones de procesado de degradación hidrolítica de VPET ....... Tabla 4.10 Viscosidad intrínseca, índice de fluidez y peso molecular de las muestras de vPET modificado hidrolíticamente

Tabla 4.11 Propiedades térmicas de las muestras de VPET modificado hidrolíticamente ....... Tabla 4.12 Valores de Módulo de Young de las muestras de VPET modificado hidrolíticamente

Tabla 4.13 Composición de las muestras de VPET modificadas químicamente ......................... Tabla 4.14 Composición y propiedades térmicas de VPET con distinta concentración de aditivo

Tabla 4.15 Muestras modificadas física y químicamente

Tabla 4.16 Composición y propiedades térmicas de las muestras modificadas física y químicamente

8

\section{Capítulo 5}

Tabla 5.1 Propiedades de la fibra de vidrio ...................................................................................... 10

Tabla 5.2 Resultados de ensayo de flexión del perfil obtenido por pultrusión en fundido ...... 114

Tabla 5.3 Resultados del ensayo de tracción del perfil (pultrusión en fundido) ........................ 115

Tabla 5.4 Propiedades de matrices de PET utilizadas en la pultrusión en polvo ...................... 116

Tabla 5.5 Propiedades de flexión de los perfiles (pultrusión en polvo) ....................................... 121

Tabla 5.6 Propiedades de tracción de los perfiles (pultrusión en polvo) .................................. 123 


\section{LISTADO DE FIGURAS}

\section{Capítulo 1}

Figura 1.1 Descripción del escenario donde se sitúa la presente investigación y descripción

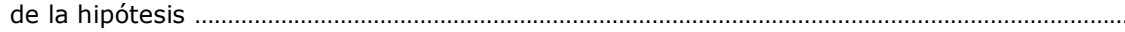

Figura 1.2 Efecto de la degradación termo-mecánica durante la extrusión en la viscosidad

Figura 1.3 Reacciones de degradación hidrolítica $\left(\mathrm{H}_{2} \mathrm{O}\right)$ y térmica $\left(\mathrm{T}^{\mathrm{a}}\right)$ del PET .....................

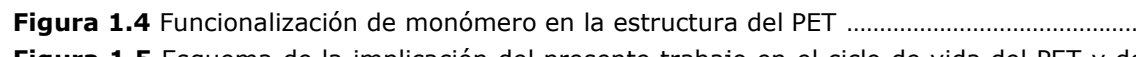
Figura 1.5 Esquema de la implicación del presente trabajo en el ciclo de vida del PET y de los materiales compuestos: de una economía lineal a una circular

\section{Capítulo 2}

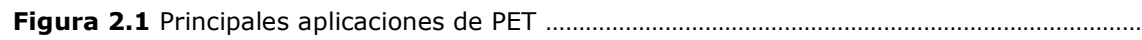

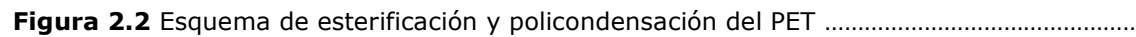

Figura 2.3 Balas de PET

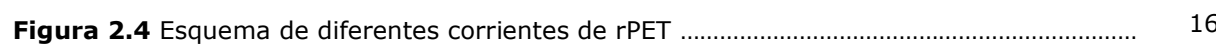

Figura 2.5 Esquema general definición de composites ............................................................ 20

Figura 2.6 Clasificación de los materiales compuestos según la morfología del refuerzo ....... 22

Figura 2.7 Comparación de composites (datos de Owens Conrning) ........................................ 23

Figura 2.8 Perfiles obtenidos a partir de procesos de pultrusión .......................................... 24

Figura 2.9 Proceso de pultrusión convencional con material termoestable ............................. 25

Figura 2.10 Diferentes métodos de pultrusión termoplástica ................................................. 26

Figura 2.11 Proceso de pultrusión termoplástica .................................................................... 27

Figura 2.12 Esquema y fotografía de PCT .............................................................................. 28

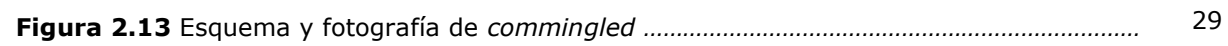

Figura 2.14 Esquema de towpreg: Fibras con polvos adheridos ............................................. 30

\section{Capítulo 3}

Figura 3.1 Extrusora corrotante de doble husillo empleada para la modificación de los

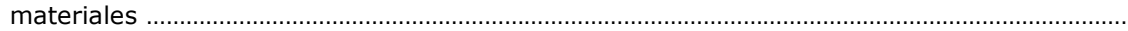

Figura 3.2 Configuración del husillo empleado para el tratamiento de los materiales .............

Figura 3.3 Máquina de inyección empleada para la obtención de probetas ............................... Figura 3.4 Equipo de DSC empleado para la caracterización térmica de las muestras de

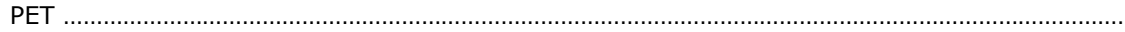

Figura 3.5 Máquina universal de ensayos Instron

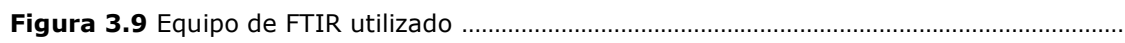

Figura 3.10 Esquema general de la cámara de impregnación situada a la salida de la

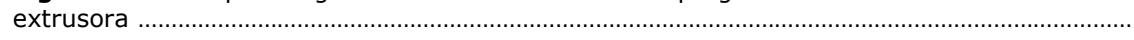

Figura 3.11 Esquema general de la cámara de impregnación ...................................................... 49

Figura 3.12 Componentes de la cámara de impregnación ........................................................... 51

Figura 3.13 Línea de pultrusión en fundido ................................................................................ 52

Figura 3.14 Imágenes y esquema general de la línea de impregnación en polvo ................... 53

Figura 3.15 Etapa de apertura de la fibra ........................................................................... 54

Figura 3.16 Etapa de impregnación de la fibra con el material en polvo ................................ 54

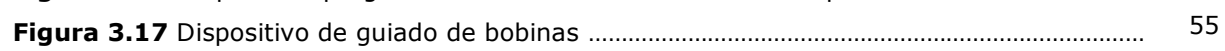

Figura 3.18 Esquema de la línea de pultrusión ..........................................................................

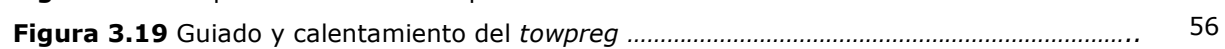




\section{Capítulo 4}

Figura 4.1 Imagen de escamas de rPET blue (a) y rPET color (b) .......................................... 62

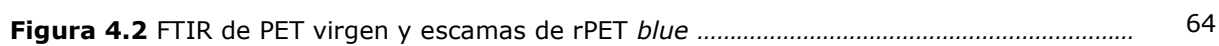

Figura 4.3 Extracción selectiva de rPET color ………………………............................................ 64

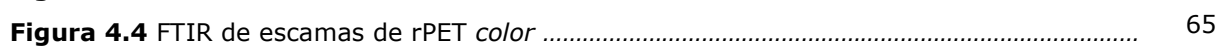

Figura 4.5 Viscosidad compleja de los materiales de PET ........................................................... 66

Figura 4.6 Peso molecular de los materiales de PET ..................................................................... 67

Figura 4.7 Fórmula molecular del aditivo utilizado para la modificación química ..................... 68

Figura 4.8 Esquema de tratamientos realizados ........................................................................ $\quad 69$

Figura 4.9 Reacciones de degradación térmica, hidrolítica y efecto del aditivo ....................... 70

Figura 4.10 Gráfico de efectos principales para los factores de extrusión estudiados ............ 72

Figura 4.11 Diagrama de viscosidad y rangos de peso molecular de los materiales de 74

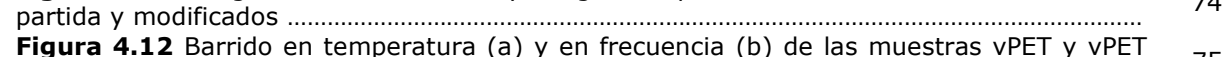

Figura 4.14 Impacto Charpy de las muestras de VPET modificado termo-mecánicamente ....

Figura 4.15 Extrusora con bomba peristáltica utilizada para la degradación hidrolítica ..........

Figura 4.16 Viscosidad compleja frente a temperatura (a) y frecuencia (b) de las muestras

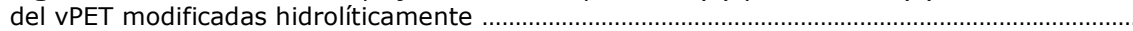
Figura 4.17 Curvas DSC de a) fusión y b) cristalización de las muestras de VPET

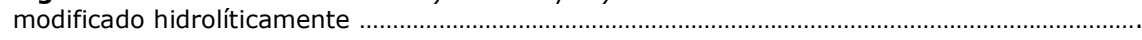
Figura 4.18 Impacto Charpy de las muestras de VPET modificado hidrolíticamente ............... Figura 4.19 Viscosidad compleja frente a temperatura de VPET con diferentes

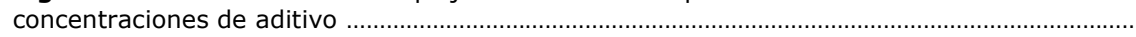
Figura 4.20 Peso molecular de vPET con diferentes concentraciones de aditivo calculado

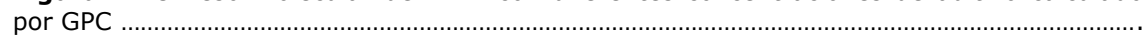
Figura 4.21 Curvas DSC a) Fusión b) Cristalización de VPET modificado químicamente .......... Figura 4.22 Módulo de Young de VPET modificado químicamente con diferentes

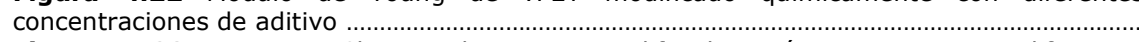
Figura 4.23 Impacto Charpy de VPET modificado químicamente con diferentes

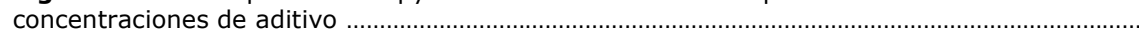

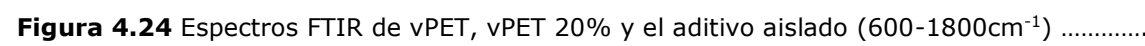

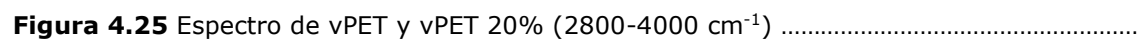
Figura 4.26 Granzas obtenidas en los distintos tratamientos de las mezclas ........................... Figura 4.27 Viscosidad compleja de las mezclas de la modificación física (a) y modificación

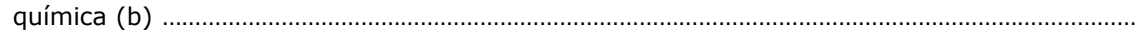

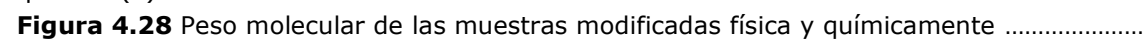
Figura 4.29 Curvas DSC de los polímeros puros y sus mezclas modificados por el método

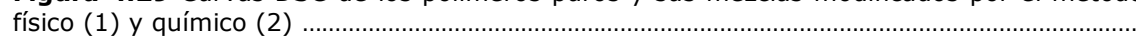

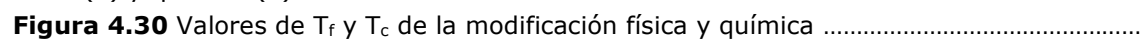
Figura 4.31 Valores de cristalinidad de la modificación física y química ...................................

Figura 4.32 Módulo de Young de mezclas modificadas física $(\boldsymbol{\square})$ y químicamente $(\bullet)$........... Figura 4.33 Impacto Charpy de mezclas modificadas física y químicamente ........................... Figura 4.34 Resistencia a la tracción de mezclas modificadas física ( $\square$ ) y químicamente (•)

\section{Capítulo 5}

Figura 5.1 Matrices modificadas reológicamente por el método físico y químico .....................

Figura 5.2 Cámara de impregnación acoplada a la línea de extrusión ......................................

Figura 5.3 Imagen de tape obtenido en la cámara de impregnación .........................................

Figura 5.4 Análisis SEM de los tapes de VPET y VPET_0.25\% obtenidos en la cámara de impregnación 5.5 Análisis SEM de los tapes de rPET y rPET_0.25\% obtenidos en la cámara de Figura 5.6 Análisis SEM de los tapes obtenidos con matrices de la modificación física y Figura $\mathbf{5 . 7}$ Volumen de matriz de los tapes obtenidos con las matrices física y

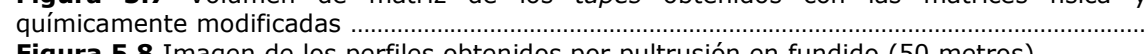

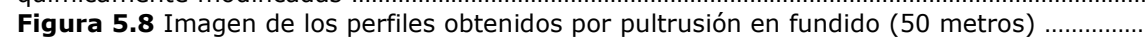

Figura 5.9 Ensayo de flexión de perfil obtenido por pultrusión en fundido ..................................

Figura 5.10 Ensayo de tracción de perfil (pultrusión en fundido) ................................................. 114 
Figura 5.11 Micrografías del perfil (pultrusión en fundido)

Figura 5.12 Viscosidad compleja de las matrices de rPET utilizadas en la pultrusión en polvo

Figura 5.13 Imagen de la reducción del tamaño de partícula

Figura 5.14 Imagen de impregnación de la fibra con el polvo

Figura 5.15 Imagen de towpreg (pre-impregnado) y de la inspección por SEM del towpreg 120

Figura 5.16 Perfiles obtenidos por pultrusión en polvo

Figura 5.17 Rotura del perfil durante el ensayo de flexión

Figura 5.18 Imágenes de SEM de perfiles obtenidos (pultrusión en polvo)

Figura 5.19 Comparación de Resistencia a la flexión de perfil termoestable convencional y perfiles termoplásticos obtenidos en la investigación con rPET

Figura 5.20 Comparación de Módulos elásticos de un perfil termoestable convencional y perfiles termoplásticos obtenidos en esta investigación con rPET

Figura 5.21 Comparación de Resistencia a la tracción de perfil termoestable convencional y perfiles termoplásticos obtenidos en la investigación con rPET 



\section{Ca pítulo 1}

Este primer capítulo pretende ser un prólogo donde se presentan las razones que han motivado la realización de este trabajo. Esta sección también servirá como guía de lectura, ya que delimitcona, consecutivamente, el objetivo general y los objetivos específicos del trabajo. Finalmente se presenta una guía de los capítulos, a través de los cuales se ha estructurado este trabajo de investigación, enfocándose en las ideas principales que en ellos se desarrollan.

\section{Motivación, objetivos y estructura de la investigación}

\subsection{MOTIVACIÓN}

En los últimos años, el consumo de los materiales plásticos ha experimentado un crecimiento exponencial en los sectores más estratégicos como son el sector del envase, la construcción y el transporte. De igual manera, estos materiales, también han empezado a cobrar vital importancia en la sanidad y las energías renovables. Tal es el consumo que, hasta la fecha, se han fabricado alrededor de 8300 millones de toneladas de plástico desde que empezó su producción en 1950, y se prevé que se alcanzarán los 34.000 millones en $2050^{1}$.

El aumento en la producción de materiales plásticos ha demostrado generar cuantiosos beneficios asociados a la economía mundial y a la creación de puestos de trabajo, sin embargo, también ha dado lugar al nacimiento de una nueva problemática: generación y acumulación de miles de toneladas de residuos plásticos. Hoy en día, de todo el plástico que se ha producido y consumido a nivel mundial, solo el $9 \%$ se ha reciclado. En cuanto al resto, el $12 \%$ se ha incinerado, y el $79 \%$, la gran mayoría, ha terminado en vertederos o en el medio ambiente ${ }^{2}$.

La acumulación de los residuos plásticos en el medio ambiente supone un riesgo para nuestra salud y la de los ecosistemas, especialmente los acuáticos. Debido a que poseen un largo tiempo de descomposición (entre 100 y 1000 años), se van acumulando en fragmentos cada vez más pequeños, contaminando todos los mares y costas del planeta. Tanto es así que, según los informes del Programa de Naciones para el Medio Ambiente (PNUMA) ${ }^{3}$, cada año acaban en los océanos entre 5 y 13 millones de toneladas de plástico, suponiendo el $80 \%$ de la basura marina, por lo que, si no cambia esta tendencia, "en 2025 nuestros océanos tendrán una tonelada de plástico por cada tres de pescado, y en 2050 habrá más plásticos que peces". Además, estos fragmentos de plástico son 
ingeridos por animales, contaminando así la cadena alimentaria de la que dependemos.

Uno de los sectores que más contribuye a esta contaminación ambiental es el sector del envase o packaging, ya que además de su elevado consumo de material plástico virgen (un $40 \%$ de todos los plásticos consumidos anualmente en Europa ${ }^{4}$ ), cuenta con una problemática añadida: genera productos de un único uso y corta vida útil. Su creciente y rápida generación de residuos constituye un grave problema social y ambiental que se ha convertido en una cuestión de gran importancia, hacia la que se están dirigiendo políticas para su gestión. Un ejemplo concreto en relación a estas normativas, es que para el año 2030 todos los envases de plástico existentes en el mercado de la Unión Europea deberán ser reutilizables o deberán poder reciclarse de manera rentable (informe publicado en 2018 por la UE relativo a la estrategia para los plásticos en la Economía Circular ${ }^{5}$ ). Dos de los mayores problemas que se encuentran a la hora de cumplir estos objetivos (envases reciclables) son la escasa separación de éstos en origen, y que, en muchos casos, los envases presentan colorantes, multi-composición y aditivos difíciles de tratar y eliminar, lo cual dificulta su reciclado e incorporación en otras aplicaciones.

El material polimérico que mejor representa esta situación es el polietilen tereftalato (PET) que, debido a su buena relación precio-propiedades, es el material por excelencia utilizado para el envasado de multitud de productos ${ }^{6,7,8}$. A pesar de que el PET es conocido por ser un material plástico reciclable que regresa a diversas aplicaciones como el envasado y el textil (sobre todo el PET cristal que proviene de botellas y envases transparentes), existen otras fracciones que no se reciclan tan eficientemente como esta última (por ejemplo, botellas de agua de diferentes colores o los envases rígidos y flexibles impermeables al oxígeno o al vapor de agua).

Cada vez con más frecuencia se crean envases de PET más complejos, como son: envases rígidos coloreados opacos o translúcidos (botellas de leche, aceite, bebidas carbonatadas), envases flexibles que presentan estampaciones (bolsas verticales que contienen alimentos en conserva) e incluso materiales multicapa mono-material (que contienen capas intermedias con materiales no poliméricos, como óxidos metálicos para la conservación de la carne). Esta corriente, es separada en el proceso de reciclaje de la fracción cristal (totalmente transparente), y hoy por hoy, debido a los tintes y aditivos que contienen, tiene muy poca salida en el mercado de las materias primas recicladas, siendo necesaria la búsqueda de nuevas aplicaciones para su revalorización ${ }^{6,7}$. 
Además del alto porcentaje de residuos que representan estas fracciones no revalorizadas (puede representar más del $20 \%$ del total de PET que se desecha anualmente), se prevé que haya un crecimiento de éstos durante los próximos años. La asociación de plásticos, Plastics Recyclers Europe (PRE), con sede en Bruselas, emitió un comunicado en 2018 expresando el crecimiento de envases de PET coloreados, debido a que muchos envasadores del sector packaging planean cambiar el material de sus productos (como son los cosméticos, champús, etc.), envasados actualmente con polietileno de alta densidad (HDPE), a materiales como el PET, que además de ser económicamente más rentable, permite crear estampaciones que con el HDPE no consiguen adquirir (por ser un material apolar). Debido a que estos productos se empaquetan típicamente en envases coloreados, el cambio podría conducir a la producción de más de 300.000 toneladas métricas adicionales de PET de $\operatorname{color}^{9,10}$. Contaminando seriamente la fracción transparente, que actualmente es un gran ejemplo de reciclaje.

Ante esta problemática, esta investigación pone su atención en trabajar con residuos de PET, específicamente con las fracciones de PET menos revalorizadas anteriormente descritas (coloreadas y multicapa mono-material), para contribuir a su reciclabilidad, dándoles usos de mayor valor añadido y de gran volumen; contribuyendo así con los objetivos de reciclaje que marcan las directivas europeas (ej. DIRECTIVA (UE) 2019/904).

Uno de los potenciales mercados por el que esta investigación apuesta que podría incorporar y utilizar este residuo como materia prima secundaria, es el mercado de composites poliméricos de fibra continua (material compuesto formado por una matriz polimérica y la fibra continua de refuerzo). El uso de PET reciclado como matriz polimérica del composite, permitiría solventar las limitaciones antes mencionadas que presenta el PET coloreado para ser incorporado en otras aplicaciones (como el envasado) ya que los pigmentos que son usados en estas fracciones de PET, no significan a priori, un impedimento técnico.

Los composites poliméricos, están siendo muy demandados por sectores tan importantes como el transporte, la construcción, el deporte y el ocio. Tanto es así que el mercado de composites en el sector del automóvil se valoró en USD 6.81 mil millones en 2016 y se prevé que duplique su crecimiento hasta los USD 13.14 mil millones para $2022^{11}$. Esto es debido a que el uso de materiales compuestos está permitiendo sustituir, en muchos casos, a los metales, disminuyendo el peso del componente sin la pérdida de propiedades ${ }^{12}$. Hasta la fecha, las resinas poliméricas termoestables han dominado el mercado en los composites, ya que presentan una viscosidad idónea que asegura la impregnación de las fibras (etapa clave en la fabricación de los composites). Sin embargo, durante los últimos años, 
el uso de estas resinas está siendo cuestionado debido entre otras razones, a su complejidad a la hora de ser recicladas ${ }^{13}$. Esta razón ha llevado a que la industria del composite haya mostrado un interés creciente en la fabricación de materiales compuestos utilizando matrices termoplásticas.

Además de su reciclabilidad la entrada de los composites termoplásticos en un sector estratégico, como es el de la automoción, se explica desde tres factores fundamentales:-1El aligeramiento, tendencia que ha marcado los desarrollos de automoción en los últimos 30 años y que se ha visto potenciada en la última década por la llegada del vehículo eléctrico. Las sucesivas restricciones en materia de emisiones, las regulaciones locales y nacionales respecto al tráfico en zonas urbanas, han transformado la tendencia de aligeramiento por movilidad limpia de cero emisiones. En esta movilidad, el peso del vehículo es un parámetro fundamental en el que los composites tienen mucho que aportar. 2La integración, los composites termoplásticos se pueden fundir y soldar, sobre-moldear y sobreimprimir, consiguiendo uniones de resistencia cercana a la del material base, con técnicas que la industria de la automoción conoce y que permiten una producción elevada. Esta es precisamente, 3la tercera razón: la producción, al ser composites que pueden moldearse por técnicas de producción continua: inyección, extrusión, pultrusión, etc.; tienen cadencias más altas que los procesos en batch (en lotes) utilizados para los composites termoestables.

En un primer intento de hacer frente a la reducción de peso en automoción, se introdujeron los composites termoestables de fibra continua llegando a fabricarse muchos componentes como capós, estructuras como el Body in White del i3, brazos de suspensión en el sexto elemento de Lamborghini etc ${ }^{14}$. Sin embargo, siempre se ha tratado de piezas en vehículos de muy baja tirada, o de piezas de geometría muy simple. En este sentido, los termoplásticos ofrecen tiempos de ciclo muy bajos (no necesitan curado) por lo que pueden adaptarse a los tiempos que demanda este sector y ampliar su uso. Sin embargo, los últimos años de investigación han permitido conocer las problemáticas a la que se enfrenta la industria, cuando ha intentado hacer el cambio de las matrices termoestables a termoplásticas, donde se ha detectado que la principal problemática se encuentra en la alta viscosidad que poseen las matrices termoplásticas, lo que dificulta la buena impregnación de la matriz en la fibra y más aún, forman un sistema con baja adhesión, que se traduce en pobres propiedades mecánicas en el composite final ${ }^{15}$.

Las soluciones hasta ahora estudiadas se han enfocado en la modificación de las líneas de moldeo existentes o en la creación de nuevas tecnologías como la fabricación de productos intermedios, como los pre-impregnados (más adelante desarrollados en el Capítulo 2). Estos logros han dado lugar a nuevos procesos de 
pultrusión que permiten incorporar materiales termoplásticos; sin embargo, persiste el problema referido a alta viscosidad de los termoplásticos; quedando así explicita, una necesidad importante de trabajar en materiales poliméricos termoplásticos reológicamente diferenciales, que permitan unir sus bondades de reciclabilidad y altas cadencias de producción, para fabricar composites con un balance de propiedades en términos de prestaciones mecánicas, rentabilidad y sostenibilidad, que realmente compitan con los composites termoestables tradicionales, para ciertas aplicaciones.

En la Figura 1.1 se hace un resumen de los problemas actuales, en relación a las dos grandes problemáticas descritas: al PET residual no revalorizado procedente del packaging y a los composites termoestables. En la Figura, también se describen las soluciones y dificultades hasta ahora encontradas en cada sector, y por último, las soluciones y retos que propone esta investigación en medio de ambos escenarios.

PET (Packaging)
COMPOSITE TERMOESTABLE

(Aeronáutica, Automoción y Construcción)

\begin{tabular}{|c|c|c|}
\hline \multirow[t]{2}{*}{ 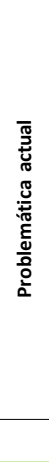 } & $\begin{array}{l}\text {-Excesiva producción de artículos de } \\
\text { usar y tirar, usados en envasado de } \\
\text { alimento, que no pueden reusarse (ej. } \\
\text { PET coloreado, multicapa simple de } \\
\text { PET). Aun cuando el PET cristal es el } \\
\text { termoplástico más revalorizado por su } \\
\text { pureza, las fracciones coloreadas o } \\
\text { multicapa no tienen el mismo valor, por } \\
\text { lo que hay un aumento considerable de } \\
\text { estos residuos en vertederos. } \\
\text { - Se demandan soluciones inminentes en } \\
\text { el marco de actuación definido por las } \\
\text { directivas de la UE. }\end{array}$ & 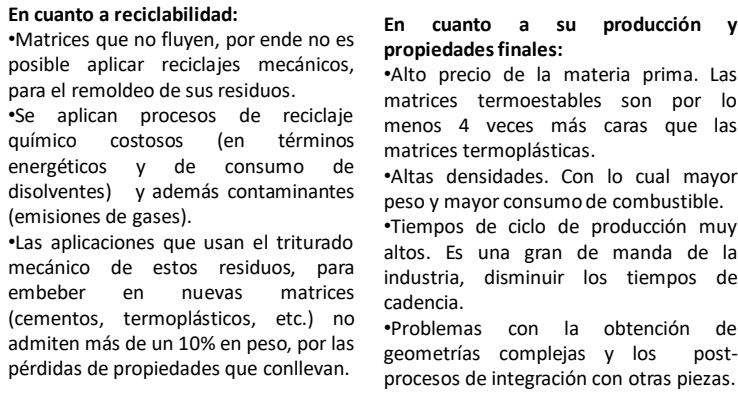 \\
\hline & $\downarrow$ & $\downarrow$ \\
\hline 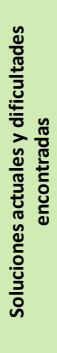 & $\begin{array}{l}\text { - Revalorización energética (incineración) } \\
\text { y con ello altas emisiones de } \mathrm{CO}_{2} \text {. } \\
\text {-Procesos de reciclaje químico para } \\
\text { obtención de monómeros de } \\
\text { polimerización. Procesos costosos, de } \\
\text { baja conversión y pureza de los } \\
\text { productosfinales. } \\
\text { - Se trabaja en el rediseño de envases y } \\
\text { en el uso de termoplásticos } \\
\text { biodegradables que resuelven la } \\
\text { problemática a futuro, pero no } \\
\text { revalorizan los residuos que existen } \\
\text { actualmente. }\end{array}$ & $\begin{array}{l}\text { Actualmente se trabaja en la idea de introducir termoplásticos vírgenes, como } \\
\text { matriz, para mejorar estos problemas, en la fabricación de composite. Sin } \\
\text { embargo, los problemas a los que se enfrenta esta solución son los siguientes: } \\
\text { - Baja compatibilidad o adhesión de las resinas termoplásticas a las fibras. } \\
\text {-Problemas de impregnación y mojabilidad de las fibras, por la alta viscosidad de } \\
\text { los termoplásticos. } \\
\text {-Baja resistencia mecánica que ha llevado al uso de termoplásticos técnicos de } \\
\text { altas prestaciones : PEEK, PEI, etc. que agravan el problema de la viscosidad y } \\
\text { encarecen, aun más, los costes. } \\
\text { - Los procesos son energéticamente más costosos porque se necesita alcanzar la } \\
\text { fusión de los termoplásticos, durante la fabricación. }\end{array}$ \\
\hline 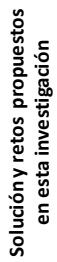 & \multicolumn{2}{|c|}{$\begin{array}{l}\text { Estudiar composites termoplásticos que usen, como matriz, la fracción poco revalorizada, de los residuos } \\
\text { procedentes del packaging (PET color y multicapa simple), a través de la investigación en estructuras } \\
\text { poliméricas de reología y funcionalidad controlada, inducidas sobre estas matrices residuales. } \\
\text { Adicionalmente, se pretende investigar en mejorar los procesos de impregnación matriz-fibra, para crear } \\
\text { sinergia entre el material reciclado modificado y el proceso de fabricación, dando respuestas, así, a las } \\
\text { demandas globales (prestaciones, rentabilidad y sostenibilidad) del composite termoestable actual y } \\
\text { dando, a su vez, un reúso de alto valor a la fracción de PET reciclado menos valorizada. }\end{array}$} \\
\hline
\end{tabular}

Figura 1.1 Descripción del escenario donde se sitúa la presente investigación y descripción de la hipótesis 


\subsection{OBJETIVOS}

\subsubsection{Objetivo general}

Una vez enmarcado el escenario, donde se desarrolla el estudio, se establece que el objetivo general es investigar en nuevas estructuras poliméricas de reología y funcionalidad controlada, inducidas sobre matrices residuales termoplásticas procedentes del packaging de baja valorización como el PET coloreado, que concentren las bondades de las matrices termoestables y termoplásticas para la obtención de composites de altas prestaciones mecánicas, alta rentabilidad y sostenibilidad, adaptando para ello, los distintos procesos de pultrusión existentes, a las matrices termoplásticas investigadas.

Para alcanzar este ambicioso objetivo, este trabajo de investigación se ha planteado dos grandes retos tecnológicos:

\section{Modificación reológica del PET reciclado (rPET) para su uso como} matriz termoplástica en procesos de pultrusión: El reto que plantea la primera fase de la investigación se enfoca en la modificación reológica del rPET con el fin de disminuir la viscosidad, sin que está implique la pérdida de propiedades mecánicas. Este reto está asociado a la obtención de poliésteres de cadenas cortas, con alta capacidad de cristalización, y así conseguir que se mantengan las propiedades mecánicas del polímero. La modificación reológica de estos poliésteres permite abordar el reto de obtener bajos pesos moleculares y distribuciones estrechas para mejorar la fluidez de estos materiales, y lograr así una mayor impregnación y mojabilidad con las fibras. Todas estas modificaciones se plantean en procesos másicos de extrusión, que permitan, a partir de los materiales originales y a través de un proceso convencional de extrusión, estar preparados para ser la materia prima de los composites termoplásticos investigados.

Este desafío ha llevado a plantear cuatro hipótesis importantes, cuyo reto es conseguir la morfología polimérica deseada que responda a las exigencias: baja viscosidad sin la pérdida de propiedades mecánicas y poder generar conocimiento acerca de la relación micro-estructura / propiedades finales.

1.1. Degradación termo-mecánica: El estudio de la degradación termomecánica tiene por objetivo analizar la influencia de los parámetros de extrusión sobre la degradación del PET y con ello, controlar la disminución de la viscosidad y la pérdida de las propiedades mecánicas durante el proceso de extrusión (Figura 1.2). 


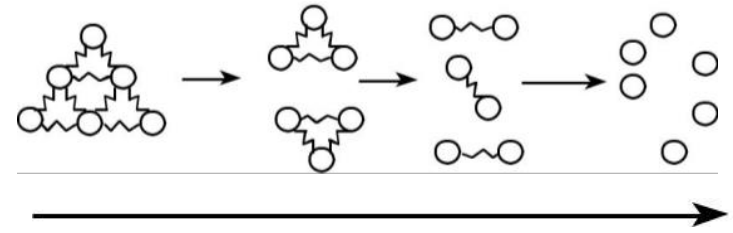

Reducción de

viscosidad

$|\mu|$

Rotura controlada de cadenas

Figura 1.2 Efecto de la degradación termo-mecánica durante la extrusión en la viscosidad

1.2. Utilizar procesos de degradación hidrolítica que, combinados con el efecto mecánico y térmico de la extrusión, conlleven a escisiones controladas de las cadenas por los puntos lábiles deseados, y a grupos terminales con alta polaridad; creando un proceso de modificación basado en la adición de vapor de agua (alineado con los términos de sostenibilidad). El gran reto se basa en conseguir los parámetros experimentales que controlen las reacciones durante la extrusión (Figura 1.3).

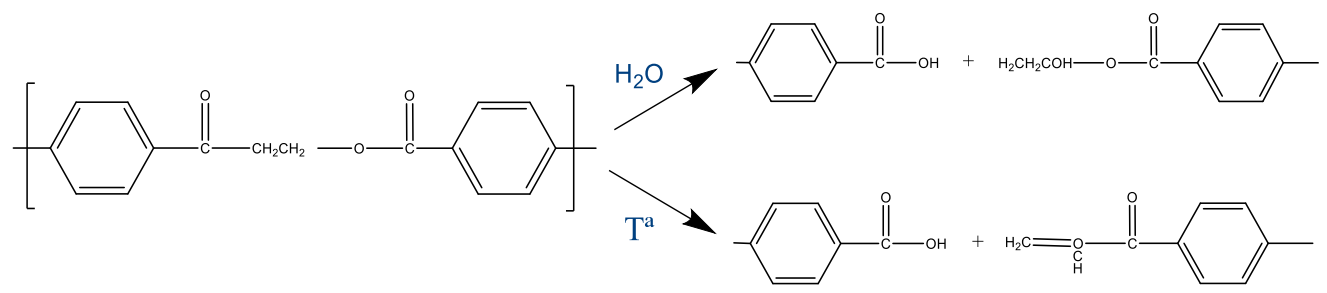

Figura 1.3 Reacciones de degradación hidrolítica $\left(\mathrm{H}_{2} \mathrm{O}\right)$ y térmica ( $\left.\mathrm{T}^{\mathrm{a}}\right)$ del PET

\subsection{Modificación física a partir de mezclas poliméricas con PET virgen} más fluido: Esta vía de modificación va a estudiar un sistema formado por dos materiales de la misma naturaleza o composición, PET residual y PET virgen que difieren en su viscosidad. La incorporación de una matriz plástica de la misma naturaleza, pero con menor longitud de cadena (mayor fluidez) permite conseguir valores de viscosidad más bajos. De estas mezclas se estudiará el comportamiento reológico, mecánico y térmico.

1.4. Modificación química: Otra vía utilizada para obtener baja viscosidad y peso molecular, se lleva a cabo por extrusión reactiva mediante la funcionalización o injerto químico de un monómero tri-funcional en la estructura del PET; de esta manera, la cadena polimérica es "cortada", seguido por el injerto de un monómero de menor tamaño. Se propone usar el ácido 5 amino isoftálico como monómero modificante o injerto, ya que por peso y reactividad se injerta en las cadenas del PET, disminuyendo el peso molecular (Figura 1.4). 


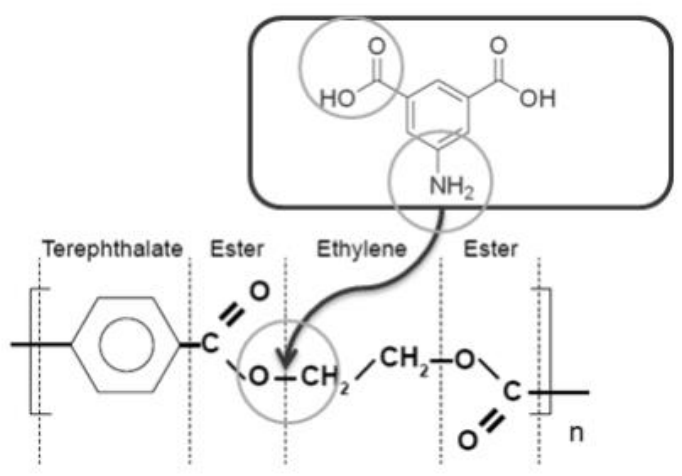

Figura 1.4 Funcionalización de monómero en la estructura del PET

Por último, se abordará una sinergia entre el sistema de modificación física y química, donde, además de tener una mezcla de materiales con diferente viscosidad, se incorpora el aditivo modificador de la viscosidad.

\section{Retos tecnológicos durante los procesos de impregnación de la fibra:}

El gran reto tecnológico que supone este proyecto, en términos de procesos de impregnación entre la fibra y el termoplástico, está orientado a introducir los materiales modificados en la investigación (reto 1); adaptando, mejorando y buscando sinergias con los procesos más punteros, como la pultrusión termoplástica y que tienen mayor repercusión en el logro de las propiedades finales requeridas en el composite (reto 2 ).

En este sentido, el trabajo de investigación ha puesto especial atención en crear sinergias entre las bondades que aporta la micro-estructura estudiada y la morfología macro-estructural que tendrán estos materiales, para maximizar el área de contacto matriz-fibra durante el proceso de impregnación. Estas morfologías macro-estructurales (pre-impregnados) están referidas a la alimentación del material estudiado y al proceso de impregnación en distintas formas: polvo o granza; para que, antes de la consolidación, ya se haya garantizado, en estado sólido, la máxima área superficial del material en contacto con las fibras.

A cada una de estas morfologías de alimentación del termoplástico: polvo o granza, hay asociado un proceso de impregnación. Por ejemplo, para alimentar termoplásticos en forma de polvo, el proceso de impregnación debe hacerse bajo las condiciones de lecho fluidizado que permita al polvo adherirse a las fibras mediante el calentamiento de estas para formar un pre-impregnado denominado "towpreg". 
En el caso de la alimentación directamente desde granza, el reto tecnológico se centra en estudiar un procedimiento de impregnación termoplástica con el uso de un cabezal de impregnación de diseño propio, acoplado a un proceso convencional de extrusión que impregne de forma homogénea las fibras, sometiendo el material a altas presiones en boquilla, debido al cambio de sección transversal. Esto le permite actuar como un líquido, rompiendo tensiones superficiales para penetrar las fibras y finalmente formar, en boquilla, un preimpregnado consolidado de sección transversal plana, conocido como "tape".

\subsubsection{Objetivos específicos}

Para abordar el objetivo general y los retos tecnológicos asociados, se marcaron los siguientes objetivos parciales dentro de cada una de las dos fases principales señaladas anteriormente:

1. Estudio de los procesos de modificación reológica de materiales residuales de PET.

- Investigación en la reducción controlada del peso molecular, a través de reacciones de escisiones de cadena, inducida por métodos hidrolíticos, térmicos y catalíticos, que le permitan regular su comportamiento reológico, para alcanzar la fluidez necesaria en la impregnación de fibras durante la fabricación del composite termoplástico.

- Estudio de morfologías diferenciales en las estructuras poliméricas del PET residual a través del injerto de monómeros funcionales ramificados, para lograr un compromiso entre propiedades mecánicas y reológicas, a través de la formación macro-estructural de estructuras tipo estrella vía extrusión reactiva.

2. Estudio de los procesos de fabricación de composites por pultrusión.

- Investigar en los principios de la tecnología de pultrusión de polvo en lecho fluidizado con los materiales investigados, para mejorar la impregnación del termoplástico residual modificado y la fibra.

- Investigar y diseñar un sistema y protocolo para la impregnación de fibras con los materiales obtenidos, a través de una cámara de alta presión de fabricación propia, en procesos de pultrusión termoplástica. 


\subsection{ESTRUCTURA DE LA TESIS DOCTORAL}

En la Figura 1.5 se presenta el esquema general de trabajo, dónde se establecen los dos retos tecnológicos planteados; retos clave para afrontar una transición de una economía lineal hacia una economía circular. En primer lugar, esta investigación busca reducir los residuos plásticos procedentes del packaging (residuos de PET de baja valorización) que actualmente acaban en el vertedero o incinerados. La segunda fase del trabajo busca conseguir composites termoplásticos funcionales y sostenibles, mediante la incorporación de materiales residuales como materia prima.

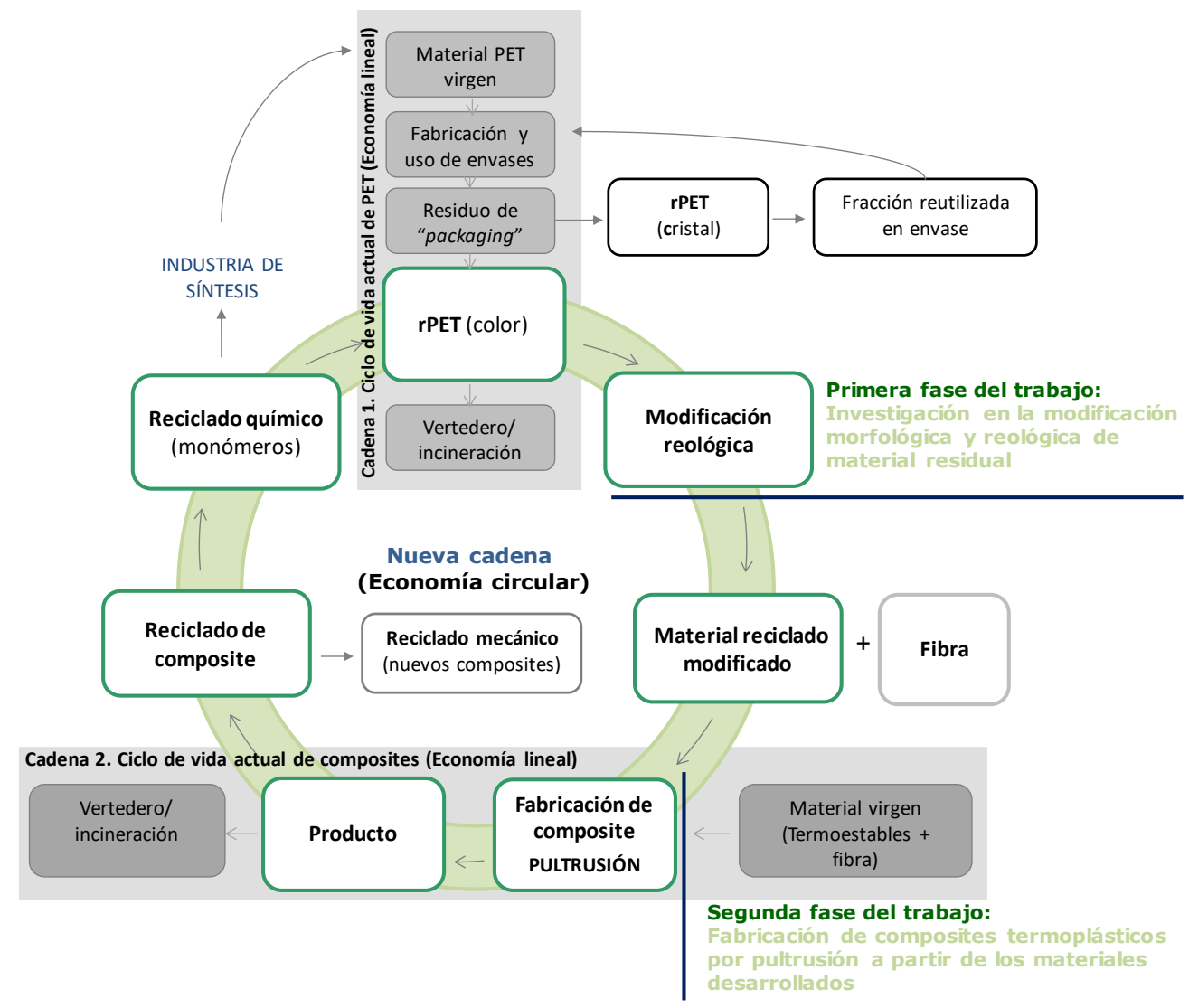

Figura 1.5 Esquema de la implicación del presente trabajo en el ciclo de vida del PET y de los materiales compuestos: de una economía lineal a una circular

La consecución de este trabajo permite avanzar en la transición de los actuales modelos económicos del PET residual y los composites termoestables (economía lineal), hacia una economía circular que permita aprovechar recursos existentes como son los materiales reciclados como materia prima, reduciendo la utilización y dependencia de materias primas vírgenes. Al mismo tiempo, la sustitución de los composites termoestables por estos termoplásticos contribuirá a reducir el uso de los anteriores, incrementando la reciclabilidad del composite. 
Los resultados de esta metodología de trabajo se verán reflejados, de la siguiente manera en los capítulos en los que se ha dividido este trabajo de investigación:

\section{Capítulo 2}

En este capítulo se presenta una revisión del estado de la técnica de los dos grandes bloques sobre los que se construye este trabajo: La matriz que se usará en la fabricación de los nuevos composites termoplásticos: Polietilen tereftalato (PET) y sus modificaciones; así como el estudio de los procesos de fabricación de estos composites y sus avances. Los estudios presentados permitirán definir el salto tecnológico que supone la consecución de esta investigación.

\section{Capítulo 3}

En este capítulo, se presentan, en primer lugar, las técnicas experimentales con las que se han llevado a cabo las modificaciones reológicas de los materiales residuales estudiados, así como las técnicas de caracterización micro y macroestructurales empleadas para el estudio de estos materiales modificados. Posteriormente, se presenta el diseño y la puesta a punto de la cámara de impregnación utilizada para la validación del proceso de impregnación de los materiales desarrollados en granza. Por último, se describen, de forma experimental, los dos procesos de pultrusión utilizados para la obtención de perfiles finales (pultrusión en fundido y pultrusión en polvo) y las técnicas utilizadas para la caracterización y validación de los mismos.

\section{Capítulo 4}

Como se explicó anteriormente, para poder utilizar materiales termoplásticos como materia prima en procesos de pultrusión, es necesario que cumplan con unas propiedades determinadas, especialmente, que posean baja viscosidad. Así pues, el presente capítulo tiene como objetivo investigar técnicas de modificación reológica que permitan modificar la viscosidad del PET de una manera controlada, sin que éstas induzcan pérdidas en las propiedades mecánicas. En primer lugar, se presentan los materiales utilizados (polímeros y aditivo) así como su caracterización completa. Posteriormente se muestra el procedimiento experimental de cada una de las modificaciones y la respectiva caracterización reológica, térmica y mecánica de las muestras obtenidas.

\section{Capítulo 5}

En este capítulo se muestra la segunda fase de este trabajo de investigación, centrada en la introducción de PET reciclado como matriz polimérica en los procesos de pultrusión. En primer lugar, se llevó a cabo el estudio de la impregnación de las matrices obtenidas en el capítulo anterior por el método físico y químico en la cámara diseñada para este fin. A continuación, se muestra 
la obtención de perfiles a partir de las dos tecnologías de pultrusión (en fundido y en polvo), así como la caracterización completa de los perfiles obtenidos. Finalmente, se comparan con las propiedades de los perfiles obtenidos con las de perfiles termoestables tradicionalmente usados.

\section{Capítulo 6}

Este último capítulo servirá para presentar las conclusiones más importantes que se derivan de esta investigación, así como el impacto y las perspectivas futuras que se plantean a partir de los resultados obtenidos.

\subsection{REFERENCIAS BIBLIOGRÁFICAS}

1. PlasticsEurope. The Circular Economy for Plastics - A European Overview. 2019:1-9.

2. European Commission. A Circular Economy for Plastics.; 2019. doi:10.2777/269031

3. ONU Programa para el medioAmbiente (PNUMA). La ONU lucha por mantener los océanos limpios de plásticos. https://news.un.org/es/story/2017/05/1378771. Published 2017.

4. Plasticseurope.org. Plastics - the Facts 2018 -. plasticseurope.org. Published 2018.

5. Comisión Europea. Residuos plásticos: una estrategia europea para proteger el planeta, defender a los ciudadanos y capacitar a las industrias. https://ec.europa.eu/commission/presscorner/detail/es/IP_18_5. Published 2018.

6. Awaja F, Pavel D. Recycling of PET. Eur Polym J. 2005;41(7):1453-1477. doi:10.1016/j.eurpolymj.2005.02.005

7. Cruz SA, Scuracchio CH, Fitaroni LB, Oliveira C. The use of melt rheology and solution viscometry for degradation study of post-consumer poly(ethylene terephthalate): The effects of the contaminants, reprocessing and solid state polymerization. Polym Test. 2017;60:236-241. doi: $10.1016 /$ j.polymertesting.2017.03.026

8. De Moura Giraldi ALF, De Jesus RC, Mei LHI. The influence of extrusion variables on the interfacial adhesion and mechanical properties of recycled PET composites. J Mater Process Technol. 2005;162-163(SPEC. ISS.):90-95. doi:10.1016/j.jmatprotec.2005.02.046

9. The Netherlands Institute for Sustainable Packaging (KIDV). Opaque PET bottles and recycling. $\quad$ https://kidv.nl/media/engelse_factsheets/factsheet-opaqueenglish.pdf?1.0.1. Published 2017.

10. ECO-MODULATION.

http://www.ecoemballages.fr/sites/default/files/files/resources/extrait_guide_des_t arifs.pdf. Published 2018.

11. Cómo y por qué el mercado de composites duplicará su crecimiento para automoción para 2022. https://www.gazechim.es.

12. Miazza NL. Principales Avances En Pultrusión y SMC.; 2010.

13. Van De Velde $K$, Kiekens $P$. Thermoplastic pultrusion of natural fibre reinforced composites. Compos Struct. 2001;54(2-3):355-360. doi:10.1016/S02638223(01)00110-6

14. Jacques K, Bax L, Vasiliadis H, Magallon I, Ong K. Polymer composites for automotive sustainability. Eur Technol Platf Sustain Chem. 2015:56. http://www.suschem.org/cust/documentrequest.aspx?DocID=998\%5Cnhttp://www. suschem.org/publications.aspx.

15. Miller AH, Dodds N, Hale JM, Gibson AG. High speed pultrusion of thermoplastic matrix composites. Compos Part A Appl Sci Manuf. 1998;29(7):773-782. doi:10.1016/S1359-835X(98)00006-2 


\section{Ca pítu I o 2}

En este capítulo se presenta una revisión del estado de la técnica de los dos grandes bloques sobre los que se construye este trabajo: La matriz que se usará en la fabricación de los nuevos composites termoplásticos: Polietilen tereftalato (PET) y sus modificaciones; así como el estudio de los procesos de fabricación de estos composites y sus avances. Los estudios presentados permitirán definir el salto tecnológico que supone la consecución de esta investigación.

\section{Polietilen tereftalato y materiales compuestos}

\subsection{POLIETILen TEREFTALATO (PET)}

\subsubsection{Historia y aplicaciones}

El Polietilen tereftalato (PET) es un polímero termoplástico de gran consumo perteneciente a la familia de los poliésteres. Este poliéster fue descubierto y patentado como polímero por dos científicos británicos Ilamados Whinfield y Dickson en $1941^{1,2}$. La primera aplicación que se le dio al PET fue como fibra en la industria textil, en la que se empleó como sustituto de las fibras naturales. Debido a sus excelentes cualidades como su resistencia a la deformación y su estabilidad dimensional, además de su facilidad de lavado y secado rápido, se abrió un amplio camino en esta industria. EI PET alcanzó mayor relevancia cuando se incorporó en los envases rígidos a partir del año 1976, sobre todo para el embotellado de bebidas carbonatadas $^{3}$. Desde su aparición hasta la actualidad el envase ha supuesto una revolución en el mercado, que continúa mejorando a medida que avanza la tecnología. De esta forma, el PET se ha convertido en el envase mayoritario de suplementos líquidos, farmacia, cosmética, entre otros (Figura 2.1).

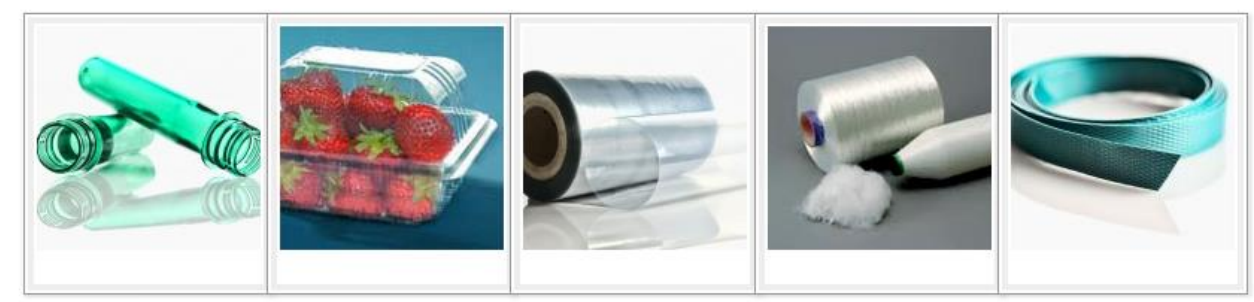

Figura 2.1 Principales aplicaciones de PET 
El uso tan extendido de este polímero se debe especialmente a su buena relación precio-propiedades; además de ser un material económicamente rentable, su capacidad para cumplir diversas especificaciones técnicas han sido las razones por las que ha alcanzado un desarrollo relevante en la producción de gran cantidad de envases. Entre sus características físico-químicas más relevantes están la cristalinidad y la transparencia, lo que no impide que también pueda ser coloreado al ser fácilmente imprimible para decorar el envase y admitir el uso de tintas. Asimismo, tiene una alta resistencia al desgaste y buenas propiedades térmicas. Actúa como barrera al $\mathrm{CO}_{2}$, al $\mathrm{O}_{2}$, y a la humedad, y al mismo tiempo es un material ideal para la fabricación de multicapas (envases flexibles de bajo espesor y alta impermeabilidad). Otra de las propiedades fundamentales del PET es su gran ligereza, lo que le otorga otra ventaja añadida en términos de transporte y reducción de emisiones de $\mathrm{CO}_{2}$, en comparación con otros materiales utilizados en envasado (por ejemplo, el vidrio).

En el mercado global existen dos grados de PET claramente dominantes que difieren en su peso molecular y cristalinidad. Cada uno de estos grados va dirigido a una aplicación concreta: fabricación de fibras o de botellas. Sólo estos dos mercados consumen la mayor parte de la producción mundial total de PET, representando un $63.5 \%$ y $30.3 \%$ del total respectivamente, mientras que la producción de film representa el $6.2 \%{ }^{4}$.

\subsubsection{Síntesis}

La síntesis del PET cuenta con dos posibles rutas de polimerización. La primera es una reacción de esterificación, donde el ácido tereftálico (TPA) reacciona con etilen glicol (EG). La segunda es una reacción de trans-esterificación, donde el dimetil tereftalato (DMT) reacciona con el etilen glicol (EG). Ambas rutas permiten la obtención del producto intermedio bis-(2-hidroxietil) tereftalato que posteriormente es sometido a procesos de pre-polimerización y polimerización (policondensación) del PET 5,6 (Figura 2.2).

\section{Esterificación y trans-esterificación}
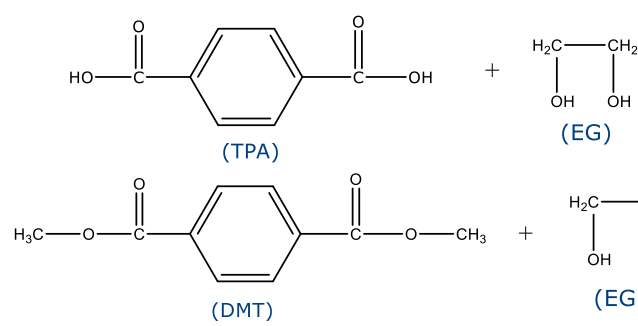

$(\mathrm{EG})$

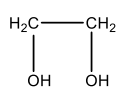

$(\mathrm{EG})$

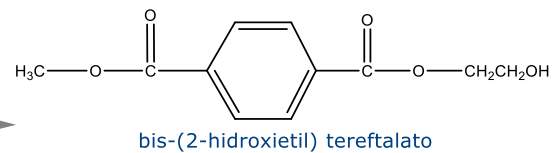

$\mathrm{HO}-\mathrm{CH}_{3}+\mathrm{H}_{2} \mathrm{O}$

metanol agua 


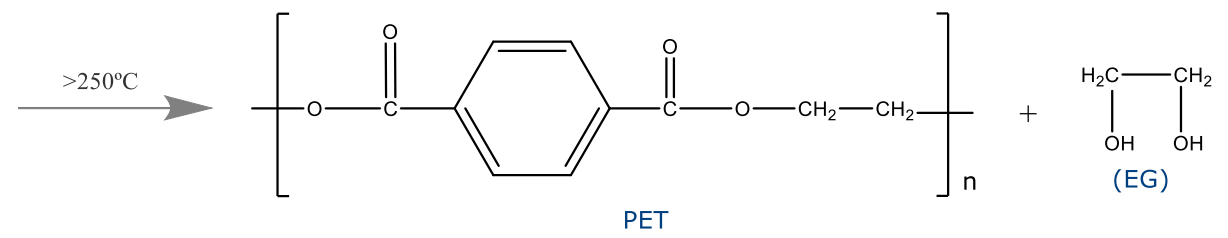

Figura 2.2 Esquema de esterificación y policondensación del $\mathrm{PET}^{7}$

\subsubsection{Reciclado}

El proceso de reciclado se puede definir como el reprocesado de un material con el propósito de integrarlo nuevamente a un ciclo productivo como materia prima. Este ciclo se cierra con la obtención de un nuevo producto, incorporando (total o parcialmente) esta materia prima secundaria. En la actualidad, de la totalidad de PET que es reciclado, un $40 \%$ se utiliza para la fabricación de laminados de envases termoformados, un $29.5 \%$ para la producción de botellas y un $15.5 \%$ para la obtención de fibras. El $15 \%$ restante se utiliza para flejes y otras aplicaciones (en 2017, según datos de PETCORE Europa ${ }^{8}$ ).

El proceso de reciclaje del PET se inicia al depositar el producto, una vez que ha finalizado su vida útil, en un contenedor de envases ligeros. Estos contenedores recogen todo tipo de materiales plásticos y envases de metal que posteriormente serán separados en la planta de selección de materiales ligeros. Estas plantas harán una selección en grupos de envases ligeros, latas y briks. Tras esta separación, el conjunto de residuos plásticos es transportado hasta una planta recicladora donde son seleccionados y separados por distintos flujos según su procedencia polimérica, con métodos de identificación infrarrojos. Tras estos procesos, los residuos de PET seleccionados se compactan físicamente para obtener una bala (Figura 2.3)7.
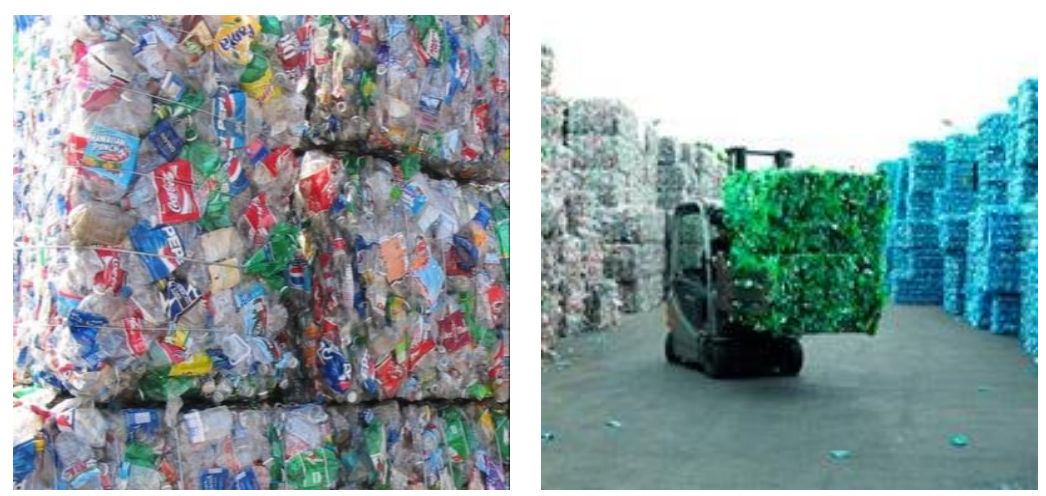

Figura 2.3 Balas de PET 
Cada una de estas balas puede estar compuesta por distintos tipos de PET reciclado (denominado $\mathrm{rPET}$ ) que proceden principalmente de residuos de botellas y de bandejas (Figura 2.4). Asimismo, las balas de botella pueden estar compuestas por PET cristal (botellas y envases transparentes) o residuos de PET que presentan colores: como el PET blue con escamas azules y transparentes o el PET color (que puede tener escamas opacas y multicolores, además de componentes multicapa). La separación de ambas corrientes de PET coloreado es primordial, ya que de no hacerlo dificultaría el reciclaje de las fracciones más limpias (PET trasparente) ${ }^{9}$.

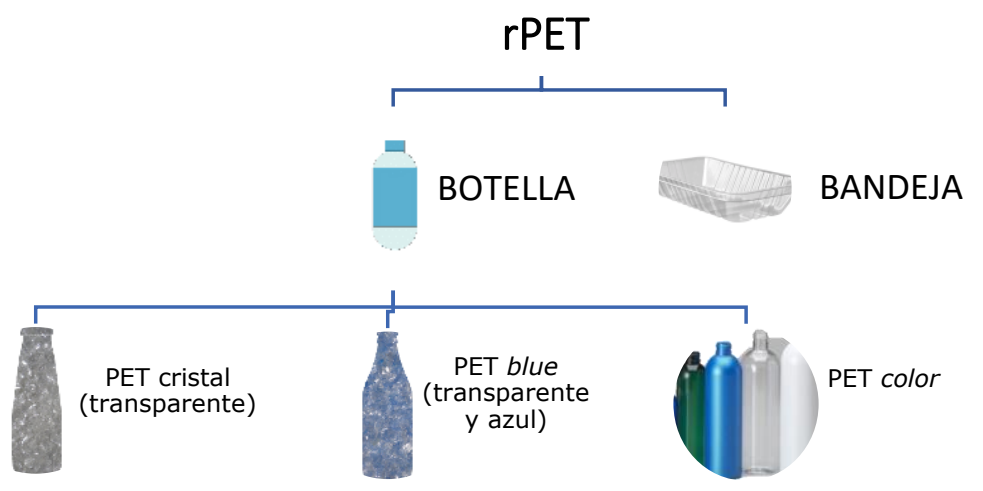

Figura 2.4 Esquema de diferentes corrientes de rPET

Tras esta separación, llevada a cabo en la planta de selección de materiales, las balas de rPET son transportadas a plantas de reciclado, donde se convierten en materia prima disponible para nuevas aplicaciones.

Es importante entender los obstáculos que se encuentran a la hora de usar el rPET como materia prima para otros procesos de producción. Una de las grandes limitaciones se encuentra en su incorporación en aplicaciones en contacto con alimentos, debido a que se requieren procesos de limpieza muy exigentes y costosos para que el material cumpla con las normativas vigentes (Real decreto 103/2009). El rPET que se utiliza para la obtención de botellas o envases en contacto con alimentos debe ser sometido a un riguroso proceso de descontaminación para asegurar que las sustancias nocivas no migran desde el envase de rPET hacia el alimento. Es por eso que, actualmente, los recicladores procesan principalmente el PET transparente (PET cristal) ya que son los residuos que menos aditivos contienen, resultando un material con unas propiedades similares a un PET virgen, lo que permite utilizarlo y obtener productos de buena calidad. 
Sin embargo, no todos los residuos de rPET se reciclan tan eficientemente como el PET cristal. Las fracciones coloreadas como el rPETblue, y en mayor medida el rPET color presentan colores que el mercado de la alimentación no puede absorber. La tinta que contienen estas fracciones disminuye la viscosidad del plástico, impidiendo que sea reutilizado en otros sectores ya que esta pérdida de viscosidad dificulta el proceso de extrusión del material.

Muchos investigadores han reportado que para poder reciclar el rPET con éxito, las escamas tienen que cumplir una serie de requerimientos mínimos (Tabla 2.1). Por lo que, otra limitación que se encuentra es que muchas balas de rPET son depositadas al vertedero por no cumplir con los requisitos establecidos.

Tabla 2.1 Propiedades mínimas de escamas procedentes de rPET para su procesado ${ }^{10}$

\begin{tabular}{cc}
\hline Propiedad & Valor \\
\hline$\mu]$ & $>0.7 \mathrm{dl} \mathrm{g}^{-1}$ \\
$\mathrm{~T}_{\mathrm{f}}$ & $>240^{\circ} \mathrm{C}$ \\
Contenido de agua & $<0.02 \mathrm{wt.} \%$ \\
Tamaño de escama & $0.4 \mathrm{~mm}<\mathrm{D}<8 \mathrm{~mm}$ \\
Contenido tintas & $<10 \mathrm{ppm}$ \\
Índice amarillamiento & $<20$ \\
Contenido Metal & $<9 \mathrm{ppm}$ \\
Contenido PVC & $<50 \mathrm{ppm}$ \\
Contenido Poliolefinas & $<10 \mathrm{ppm}$ \\
\hline
\end{tabular}

En la actualidad se aplican dos procesos principales para reciclar PET postconsumo y convertirlo en una materia prima secundaria: químico y físico ${ }^{6}$. El reciclado químico se refiere a los procesos que pueden descomponer o despolimerizar los polímeros en sus monómeros originales u otras materias primas químicas. Las materias primas pueden entonces ser reutilizadas para la síntesis de nuevos polímeros u otros productos químicos. Muchos de estos procesos se encuentran, todavía, en la etapa pre-comercial; sin embargo, están generando interés como sustituto de las fuentes de materias primas no sostenibles. La desventaja de los procesos de reciclaje químico es su alto coste, debido a la alta pureza que se requieren en los monómeros de síntesis. Además del uso elevado de disolventes contaminantes. Los principales procesos de despolimerización del PET son los siguientes:

- Glicólisis: despolimerización parcial por acción del etilenglicol. La mezcla obtenida se somete tras una purificación a la polimerización con resina virgen por adición de polímero con monoetilenglicol (MEG) en condiciones controladas para generar BHET (bis 2-hidroxietil tereftalato), que puede ser 
seguido de una hidrólisis para generar tereftálico/dimetil tereftalato (TPA/MEG).

- Hidrólisis: despolimerización total por medio del agua en presencia de ácidos o álcalis. Este método presenta dificultades a la hora de purificar el ácido Tereftálico que se obtiene en la reacción.

- Metanólisis: despolimerización completa del PET por acción del metanol. Consiste en una trans-esterificación para generar dimetil tereftalato (DMT) y monoetilenglicol(MEG), los cuales tras su purificación son polimerizados nuevamente.

El reciclaje físico, o comúnmente conocido como reciclado mecánico, consiste en la eliminación de la contaminación, mediante la clasificación, lavado y secado, y el reprocesado mediante extrusión. A partir del proceso de reciclaje mecánico, se pueden obtener películas, láminas, material triturado o granza (pellets), que se utilizarán en la fabricación de nuevos productos. Las ventajas del reciclaje mecánico es que es un proceso relativamente sencillo, respetuoso con el medio ambiente y requiere de una baja inversión.

La principal desventaja en este tipo de reciclado es la pérdida de propiedades mecánicas que experimenta el PET tras ser procesado. La pérdida de propiedades viene dada por las reacciones de degradación que experimenta durante su procesado (reacción de degradación térmica e hidrolítica) ${ }^{11}$. Ambas reacciones son inherentes al procesado del material e inducen la escisión de cadena del polímero, provocando la disminución del peso molecular (Mw) y la viscosidad. El peso molecular determina las propiedades mecánicas tales como rigidez, resistencia, viscoelasticidad y tenacidad. Si el Mw disminuye sustancialmente, la temperatura de transición vítrea y las propiedades mecánicas son demasiado bajas para que el material polimérico sea válido en aplicaciones comerciales.

Esto ha conducido a que en los últimos 20 años se hayan buscado tecnologías para encontrar formas de mantener la viscosidad durante el procesamiento del PET y así evitar esta pérdida de las propiedades mecánicas que conlleva. Estas tecnologías se basan en aumentar el Mw del PET a través de "Extensores de cadena", que son pequeños injertos de cadenas alifáticas que minimizan y contrarrestan este fenómeno ${ }^{12}$. Las extensiones de la cadena, ramificaciones, y/o reacciones de entrecruzamiento que tienen lugar entre las moléculas de PET y los modificantes han sido las bases para la mejora de las propiedades reológicas deseadas. Así, al aumentar la longitud de cadena se obtienen mayores Mw y consecuentemente el aumento de la viscosidad. Para este fin, el uso de modificantes como los reactivos di- y poli-funcionales han sido ampliamente estudiados. Los modificantes de cadena pueden ser introducidos en el PET por 
varias vías: reactores de policondensación, en estado sólido y por extrusión reactiva ${ }^{13}$.

La descripción de estos estudios demuestra que hasta ahora, los procesos de modificación del PET reciclado han estado enfocados al aumento del peso molecular y la viscosidad de estos materiales, para poder ser reutilizados en aplicaciones como por ejemplo, el blowmolding. Además, los estudios de la cinética de las reacciones de degradación del PET, enfocados a su reciclado mecánico o químico, han demostrado que éstas provocan una rotura totalmente al azar de los enlaces del PET, induciendo una pérdida dramática de las propiedades mecánicas. A diferencia de los estudios expuestos en los que buscan aumentar la viscosidad y el peso molecular, este trabajo busca disminuir la viscosidad del PET por métodos que no impliquen la pérdida de propiedades mecánicas.

Finalmente, ni las investigaciones en procesos de reciclaje químico ni físico del PET virgen o residual se han enfocado hasta ahora en conseguir un método de modificación hidrolítica controlada y una funcionalización en la estructura del PET, que permita modificar los parámetros reológicos deseados, manteniendo la macromolécula, para ser usada en un proceso de impregnación de fibras en la fabricación de composites termoplásticos. Por lo que el estudio que plantea esta investigación es un reto altamente innovador en el campo de la ciencia de los materiales y no se ha encontrado antecedentes previos de investigaciones análogas.

A partir de estas bases se plantea el primer reto al que se enfrenta esta investigación: Modificación reológica del PET reciclado (rPET) para su uso como matriz termoplástica en procesos de pultrusión. 


\subsection{MATERIALES COMPUESTOS}

\subsubsection{Aspectos generales}

Los materiales compuestos o composites poliméricos son materiales definidos por la combinación de una matriz polimérica (termoestable o termoplástica) que actúa como aglutinante de un refuerzo compuesto por fibras (carbono, vidrio, aramida, fibras naturales,... $)^{14}$. En la Figura 2.5 se muestran de forma esquemática las posibles combinaciones matriz-refuerzo-aditivos que puede componer un composite.
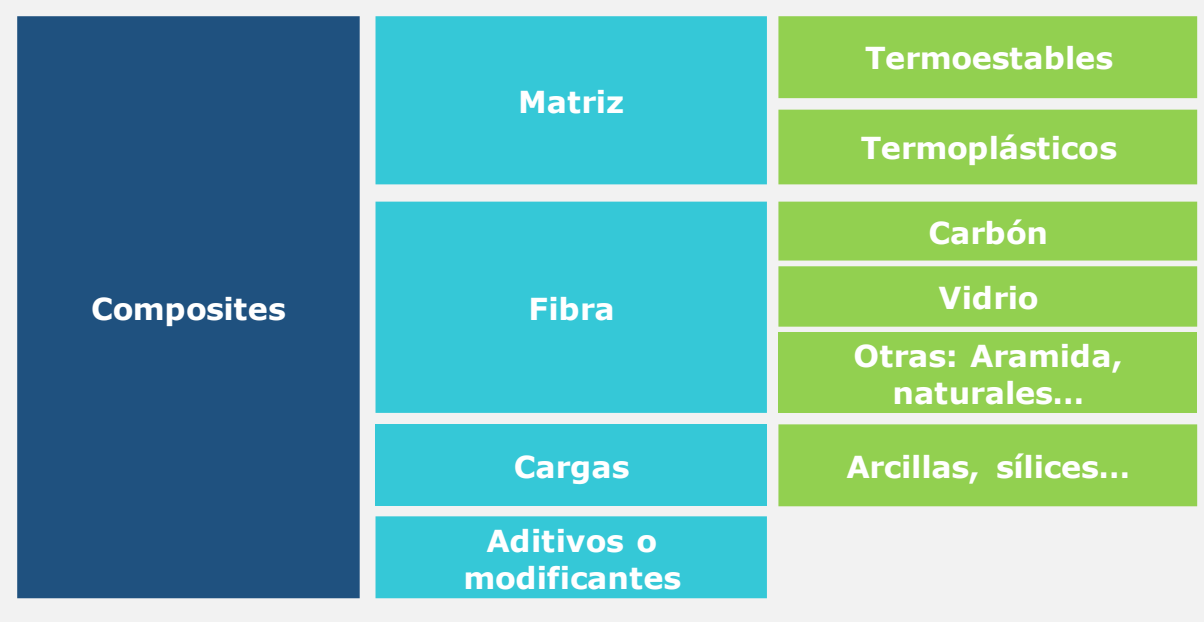

Figura 2.5 Esquema general definición de composites

El uso de los composites poliméricos se ha extendido durante los últimos años en la producción de componentes en sectores muy variados como la aeronáutica, medicina, ocio, ingeniería naval y civil, automoción, etc. El amplio abanico de aplicaciones es consecuencia de las grandes ventajas que ofrecen frente a los materiales de ingeniería tradicionales como, por ejemplo, la reducción de peso que ofrecen al sustituir a los metales sin pérdida de propiedades. Debido al continuo desarrollo de fibras de alta resistencia, de nuevos polímeros y a la evolución de los procesos de fabricación, la industria de los materiales compuestos sigue creciendo exponencialmente.

La composición exacta de los composites va a depender de su aplicación final, siendo los factores más relevantes a considerar: la fibra (tipo, volumen y orientación), la naturaleza de la matriz (termoestable o termoplástica), las condiciones de servicio, el coste del producto, el proceso de fabricación y el volumen de producción. 


\section{Matrices poliméricas: termoestables o termoplásticas}

Las funciones principales de la matriz son transferir la tensión y la carga entre las fibras, actuar como agente aglutinante que adhiere las fibras y proteger las fibras de daños ambientales y mecánicos. Se pueden diferenciar dos tipos de matrices poliméricas: termoestables y termoplásticas.

Las matrices termoestables cuentan con la particularidad de ser líquidas en su estado inicial permitiendo alcanzar una adecuada impregnación de las fibras y facilitando el proceso de fabricación. Es en el proceso de curado, por acción del calor y presión, cuando se produce la solidificación de éstas por la formación de enlaces covalentes entre sus moléculas (entrecruzamiento). Estos enlaces forman una estructura de red tridimensional, lo que hace que sean difíciles de ser revertidos o reformados, careciendo de la posibilidad de volver a fundir. Su incapacidad de volver a fundir hace que su reciclaje sea muy complejo. Las matrices termoestables también cuentan con otras propiedades deseables como una excelente resistencia a disolventes y corrosivos, estabilidad térmica (soportan altas temperaturas sin perder rigidez) y un excelente acabado.

Las matrices termoplásticas, a diferencia de las termoestables, son sólidas a temperatura ambiente, pero son capaces de fluir por acción de la temperatura. Es por ello, que para su procesado se funden para ser moldeadas y se vuelven rígidas cuando se enfrían significativamente por debajo de su temperatura de transición vítrea $\left(T_{g}\right)$. En la solidificación se forman enlaces débiles de Van der Waals entre las cadenas de monómeros de manera reversible, favoreciendo su capacidad de ser refundidas y remoldeadas, lo cual hace que sean potencialmente más fáciles de reciclar que las matrices termoestables anteriormente expuestas. Además de esta capacidad de fundir, es importante destacar que muchas matrices termoplásticas tienen una mayor resistencia al impacto que otras matrices termoestables comparables y permiten tiempos de ciclo de moldeo más rápidos, debido a que no requieren de procesos de curado ${ }^{15}$.

Las ventajas de usar matrices termoplásticas, como son los tiempos de ciclo más cortos así como su potencial reciclaje posterior a su vida útil ${ }^{16}$, hace que en los últimos años la investigación en el campo de los composites se haya centrado en la sustitución de las resinas termoestables por matrices termoplásticas ${ }^{15}$.Uno de los nuevos materiales termoplásticos introducidos son las matrices acrílicas. Estas se caracterizan por su transparencia, superficie brillante, resistencia a las condiciones climáticas y su alta dureza. Debido a todas estas características, estos materiales se utilizan en un gran número de aplicaciones, como en automóviles, electrodomésticos o construcción. La novedad de esta familia de materiales reside 
en su adaptación al campo de los composites termoplásticos. Por una parte, se ha conseguido que sean resinas acrílicas con naturaleza termoplástica (en cuanto a posibilidad de termo-conformado, reprocesado, reciclado y soldadura), y a la vez presentan propiedades mecánicas similares a las resinas termoestables comúnmente utilizadas en el sector ${ }^{17}$.

\section{Refuerzos}

Los refuerzos confieren la mayor parte de las características mecánicas del material compuesto como la resistencia y la rigidez. Otro criterio de clasificación de los composites está referido a la forma que posee el refuerzo utilizado. Como se muestra en la Figura 2.6, existen dos clases de materiales compuestos según este criterio: particulados o compuestos por fibras (continuas o discontinuas).

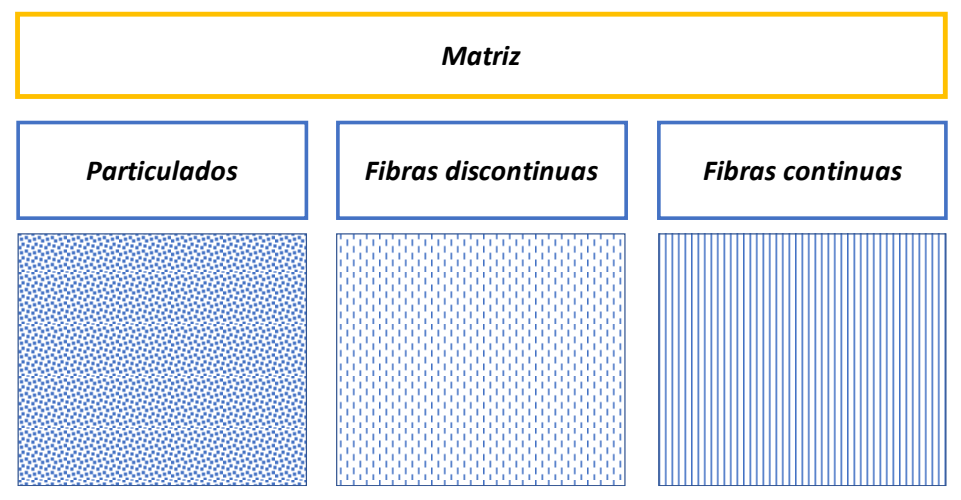

Figura 2.6 Clasificación de los materiales compuestos según la morfología del refuerzo

Cada uno de estos sistemas presenta unas propiedades únicas y dependiendo de su aplicación potencial, pueden ser divididos en categorías especificas ${ }^{18}$. Los composites particulados se caracterizan por presentar los refuerzos en forma de partículas suspendidas en la matriz. Estas partículas son equiaxiales, es decir, son aproximadamente iguales en tamaño en todas las direcciones. Con el empleo de elementos de refuerzo en forma de partículas, el aumento de propiedades que se consigue no es muy elevado. No obstante, el comportamiento mecánico en este tipo de materiales suele ser isótropo, y son los materiales menos costosos y de más fácil fabricación en comparación con otros materiales compuestos.

Los refuerzos fibrosos se componen por fibras continuas o discontinuas suspendidas en la matriz. La clasificación de las fibras discontinuas puede basarse en la relación longitud/diámetro como fibras cortas y largas, o en la orientación y/o arquitectura de las fibras. Estos materiales compuestos de fibra discontinua pueden ser producidos de tal manera que las fibras sean orientadas aleatoriamente 0 tengan alguna orientación definida. Los composites de fibra continua se 
caracterizan por tener una relación muy alta de longitud/diámetro, estos materiales se emplean cuando se requieren elevadas propiedades mecánicas al ser muy resistentes y rígidos. Esto puede verse en la Figura 2.7, donde se muestra las propiedades mecánicas que aporta cada tipo de refuerzo.

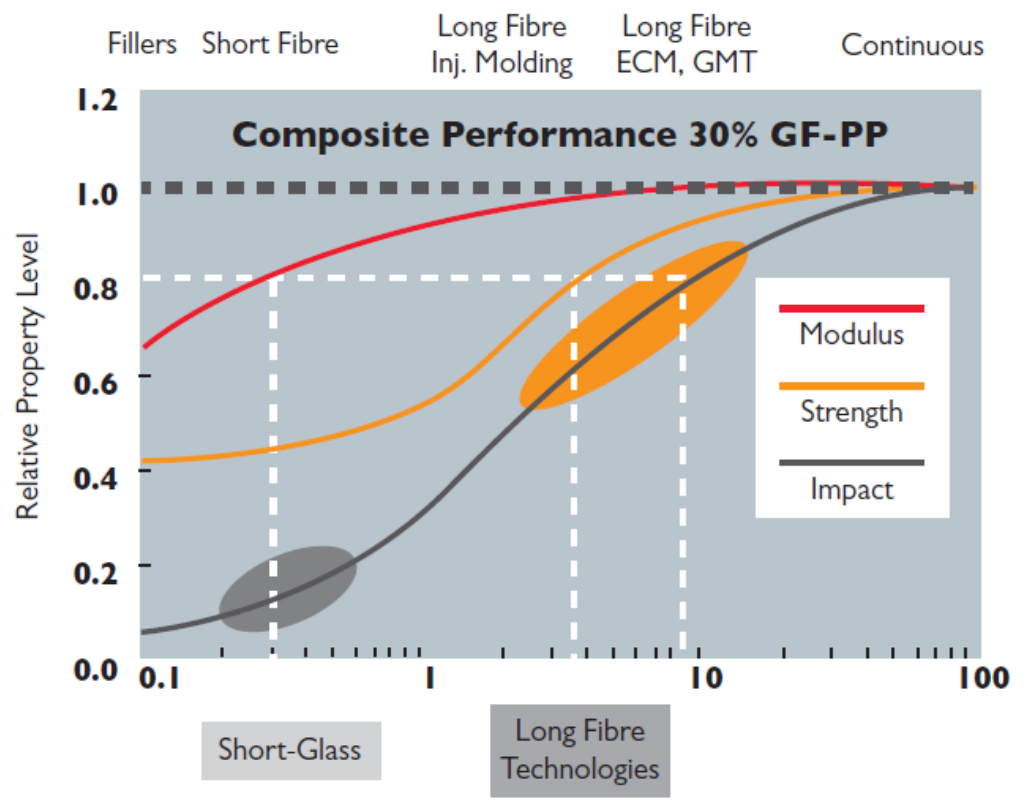

Figura 2.7 Comparación de composites (datos de Owens Conrning) ${ }^{19}$

Los composites de fibra continua han despertado especial interés industrial en los últimos años. Esto se ha debido a la rápida expansión del uso de estos composites en los sectores de automoción, construcción, deporte, ocio y otras industrias de producción en serie que requieren altas prestaciones mecánicas en sus productos.

La fabricación de los composites de fibra continua se lleva a cabo mediante conformados, formados por varias capas de fibras en forma de filamentos continuos dispuestas en forma unidireccional o formando tejidos. El termo-conformado ha sido, durante mucho tiempo, la técnica tradicional más utilizada y recurrida para el procesado de composites termoplásticos de fibra continua, además de la infusión, Resin Transfer Moulding (RTM) o la pultrusión. 


\subsubsection{Procesos de obtención de composites de fibra continua: Pultrusión}

En los últimos años, el proceso de pultrusión ha experimentado un notable crecimiento en la industria de los composites de fibra continua, debido a su rentabilidad, automatización y alta calidad de los productos ${ }^{20}$. La pultrusión es un proceso continuo, automático y de molde cerrado, especialmente diseñado para altos volúmenes de producción, en cuyo caso es económicamente muy rentable para hacer perfiles de sección transversal continua (Figura 2.8). A partir de esta tecnología, se obtienen materiales compuestos estructurales, donde la elevada resistencia los hace idóneos para aquellos casos donde no solo es importante el peso o el aislamiento eléctrico sino también, los requerimientos mecánicos.

Desde su primera producción en 1956, el consumo de perfilería ha ido aumentando en una larga lista de sectores productivos (construcción e infraestructura, náutica, energías renovables, deportes y ocio, automoción y transporte y aeronáutico). Hoy en día, el proceso de pultrusión es ampliamente utilizado para la fabricación de palas de aerogeneradores, perfiles de ventanas, paneles de puertas y barras de refuerzo para hormigón ${ }^{21}$. Una de las principales características de este proceso es la gran variedad de materiales que se pueden utilizar (diferentes tipos de matrices, fibras, cargas, etc.) cubriendo un amplio espectro de propiedades del composite final ${ }^{22}$.

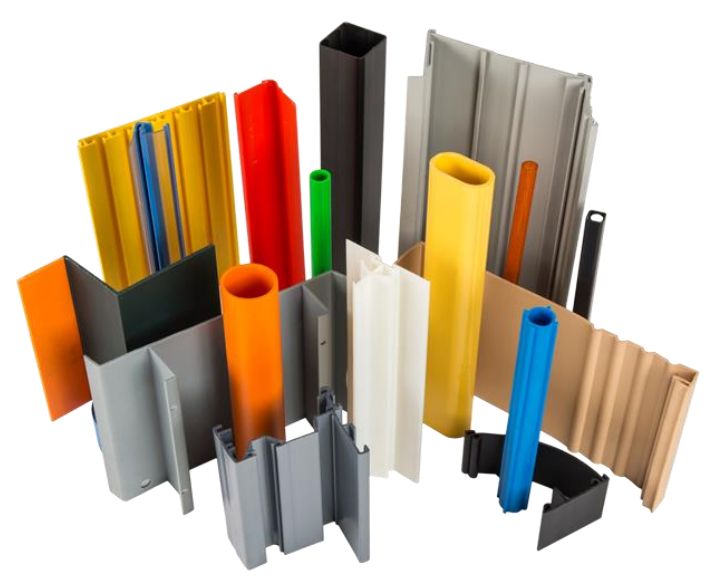

Figura 2.8 Perfiles obtenidos a partir de procesos de pultrusión ${ }^{23}$ 


\section{Pultrusión convencional}

El proceso de pultrusión convencional es aquel que emplea matrices termoestables para la fabricación de perfiles pultruidos. El tipo de matriz termoestable que se emplea, al igual que la fibra, es utilizada de acuerdo a los requerimientos del producto, concretamente más del $90 \%$ se componen de poliéster termoestable y fibra de vidrio. Cuando se requieren propiedades específicas como una alta resistencia a la corrosión se usan matrices de vinil éster, para la obtención de altas propiedades mecánicas y eléctricas se usan las matrices de epoxi y cuando se necesita combinar una alta resistencia a la temperatura y altas propiedades mecánicas se usan las matrices epoxi combinadas con fibras de aramida o de carbono ${ }^{14,24}$.

El proceso de pultrusión termoestable (Figura 2.9) comienza con la impregnación de las fibras y la matriz mediante la introducción de las mismas en un baño situado a la entrada del molde (Resin impregnation bath). Las matrices termoestables, tal y como se ha indicado anteriormente, presentan una viscosidad inicial muy baja, lo que permite una buena impregnación de la fibra. Una vez que las fibras se encuentran impregnadas, un sistema de tirado las lleva hacia un molde a alta temperatura donde se produce el curado (solidificación) de la matriz.

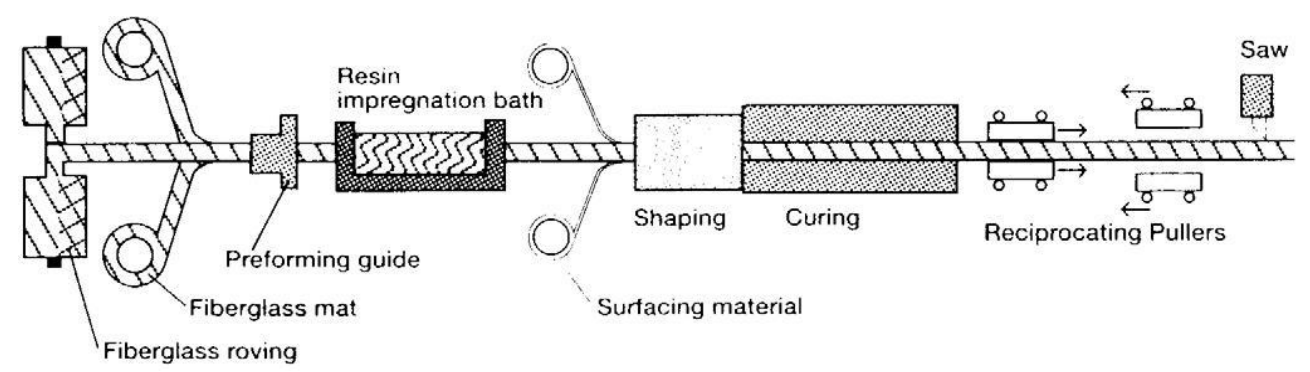

Figura 2.9 Proceso de pultrusión convencional con material termoestable 25

Como se explicó anteriormente la industria del composite está avanzando hacia la sustitución de las matrices termoestables por termoplásticas. Estos avances supondrían grandes ventajas en los procesos de pultrusión por varios motivos: reduciría en más de un $50 \%$ los tiempos de ciclos (no se necesita curado, solo fundirla), permitiría la reciclabilidad de los perfiles finales, así como mejoraría la moldeabilidad y reparabilidad post-proceso ${ }^{26}$.

El principal problema que se encuentra a la hora de integrar las matrices termoplásticas en los procesos de pultrusión radica en la alta viscosidad en fundido (problemas reológicos) que presentan estas matrices, en comparación con las resinas termoestables tradicionalmente utilizadas en el proceso de pultrusión. 
La viscosidad de las matrices termoplásticas (entre 500 y $5000 \mathrm{~Pa} \cdot \mathrm{s}$ en comparación con las resinas termoestables alrededor de $100 \mathrm{~Pa} \cdot \mathrm{s}$ ) dificulta en gran medida, la impregnación de las fibras. Como resultado de una adhesión fibra/polímero deficiente se obtendrían perfiles con pobres propiedades mecánicas. En este sentido, los últimos años de investigación enfocados en la introducción de matrices termoplásticas en pultrusión se han centrado en solventar el problema asociado a la viscosidad de los termoplásticos, mediante en el diseño de nuevos procesos de pultrusión (que sustituyan el baño utilizado para impregnar las resinas termoestables) y permitan conseguir una buena interacción entre la matriz termoplástica y la fibra.

\section{Pultrusión termoplástica}

En la Figura 2.10 se muestran los métodos de pultrusión termoplástica hasta ahora desarrollados, en los que se contemplan dos tipos de técnicas: Pultrusión termoplástica reactiva, conocida como RIM (Reaction Injection Molding) y la pultrusión no reactiva (a partir de pre-impregnados).
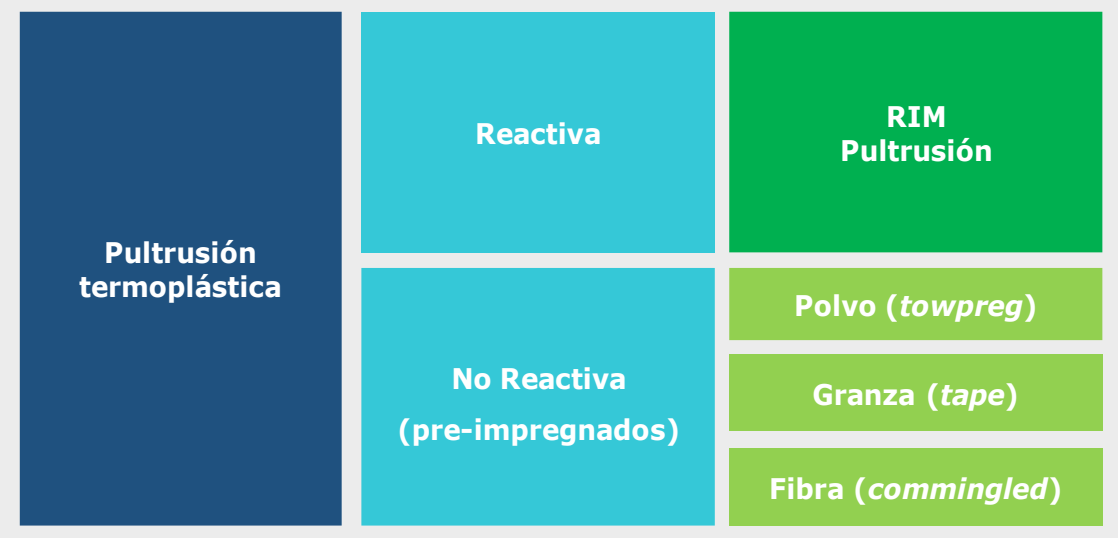

Figura 2.10 Diferentes métodos de pultrusión termoplástica

Por un lado, se ha desarrollado la pultrusión termoplástica reactiva, en la cual la impregnación se produce al mismo tiempo que se está formando (polimerizando) el propio polímero. El estado líquido en el que se encuentra el polímero antes de polimerizar, asemejándose a las matrices termoestables antes del curado, permite alcanzar una buena impregnación con la fibra. Esta técnica ha sido utilizada a partir de polímeros termoplásticos como las poliamidas (PA6, PA66, PA12) mediante reacciones polimerización aniónica ${ }^{27-29}$. 
Otra de las nuevas tendencias en el campo de los composites termoplásticos es el uso de materiales pre-impregnados termoplásticos, que son aquellos formados por una fibra continua y un polímero termoplástico adherido o en contacto directo con ésta, de forma que se comporta como un material intermedio más o menos uniforme y que está preparado para facilitar su posterior procesado. Durante los últimos años se han desarrollado líneas de pultrusión donde las fibras no son mojadas o impregnadas en un baño, sino que utilizan estos pre-impregnados que garantizan el contacto físico entre la fibra y el polímero antes de ser consolidados ${ }^{30-}$ 32. Finalmente, el perfil final es obtenido mediante el guiado de estos preimpregnados al molde de consolidación (Figura 2.11) 33 .

\section{Consolidación}

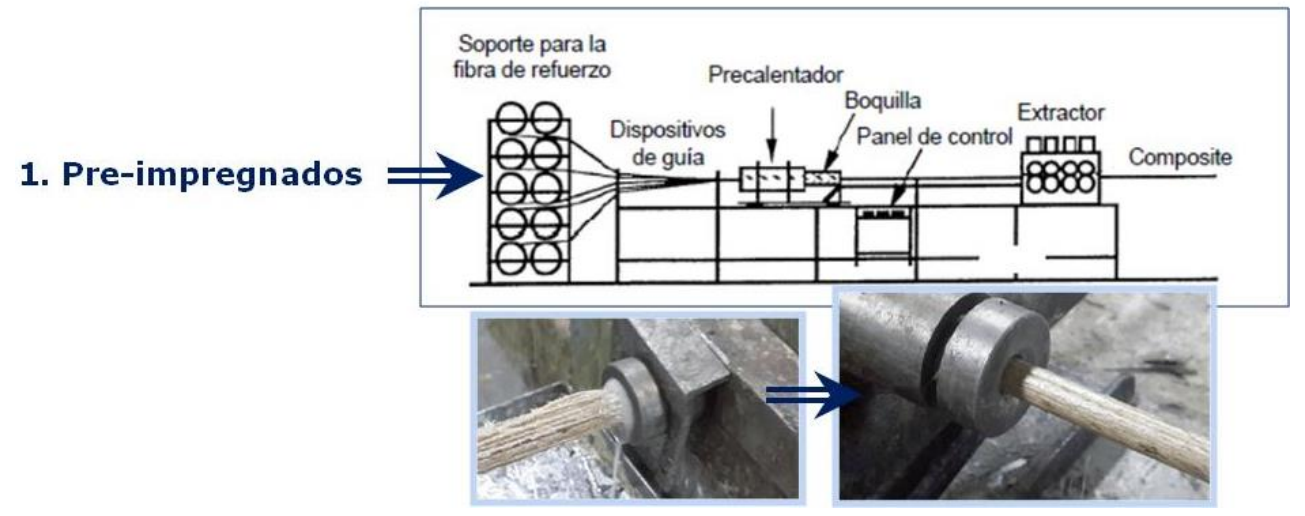

Figura 2.11 Proceso de pultrusión termoplástica25

\section{Tipos de pre-impregnados}

El término pre-impregnado engloba a distintos semi-elaborados, en función del proceso de fabricación y de su forma final. Las metodologías más recientes y aceptadas en la fabricación de pre-impregnados termoplásticos son los tapes o preconsolidados (PCT), el commingled y el towpreg, cuya principal diferencia es la forma física en la que se encuentra la matriz (en granza, fibras o en forma de polvo respectivamente) $)^{15}$.

1) El tape o la cinta pre-consolidada (PCT), es un tipo de pre-impregnado que consiste en una mecha formada por un conjunto de fibras, comúnmente conocido como roving, el cual ha sido impregnado por el polímero fundido y al que se le ha dado forma de cinta plana (Figura 2.12). A diferencia de los otros preimpregnados, el tape es un tipo de semi-elaborado donde se utiliza el material en forma de granza y se presenta como un perfil plano rígido con las fibras totalmente impregnadas por la matriz. 

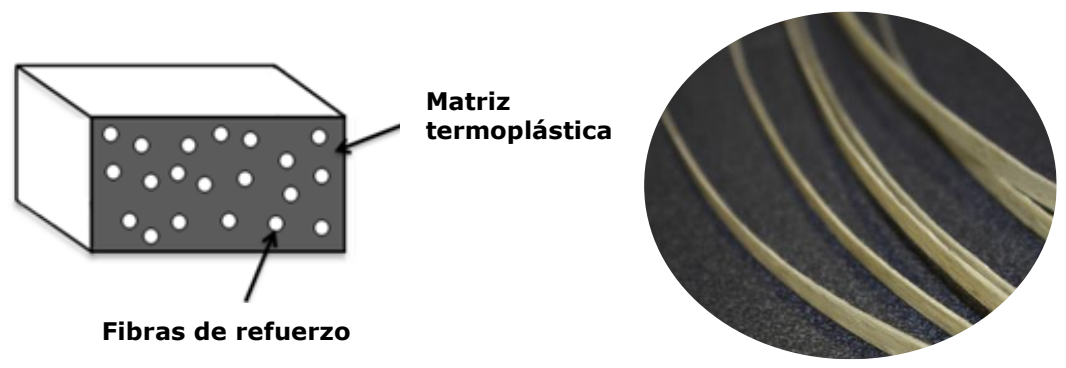

Figura 2.12 Esquema y fotografía de $\mathrm{PCT}^{34}$

La tecnología de obtención del tape cuenta con un sistema que sostiene las mechas de fibra o roving, un sistema de guía para transportar la fibra a la unidad de impregnación, una extrusora para fundir y alimentar el polímero fundido a la unidad de impregnación, la unidad de impregnación y, posteriormente, una unidad de enfriamiento y un dispositivo donde se recoge el pre-impregnado ${ }^{35}$. De todos ellos, el elemento más importante es la unidad de impregnación, donde se introducen las fibras de refuerzo, se esparcen y se impregnan con el polímero fundido. La impregnación se logra mediante la acumulación de presión que actúa sobre el polímero fundido, atrapado entre los elementos de expansión de la unidad y las mechas de fibra.

Los trabajos más recientes en el desarrollo de tapes se basan en polímeros técnicos como el PEEK y fibra de carbono que posteriormente se usan en procesos de encintado automático, muy utilizados en la obtención de componentes aeronáuticos ${ }^{31}$. En otros trabajos ${ }^{36}$ se obtienen PCT de polipropileno y fibra de vidrio o de carbono para introducirlos, posteriormente, en una línea de pultrusión. Las propiedades mecánicas obtenidas en los perfiles compuestos pultruidos a partir de estos tapes son considerados adecuados para aplicaciones de ingeniería común o estructural ${ }^{30}$.

Otra vía de investigación en la obtención de perfiles a partir de tapes se centra en el uso de fibras naturales, este es el caso del trabajo de McGregor $^{34}$ donde estudian las propiedades mecánicas y la capacidad de obtener tapes de fibra de lino con poliamida, usando una nueva variante en la unidad de impregnación (un spray que dosifica material en continuo).

2) El commingled es un tipo de pre-impregnado termoplástico que consiste en la combinación de fibras de refuerzo y fibras termoplásticas, formando así un roving mixto de refuerzo y matriz (Figura 2.13). La intrínseca combinación de fibras de refuerzo y matriz dentro de un mismo roving, facilita que se produzca una buena impregnación de las fibras durante el proceso de consolidación ${ }^{37}$. Otra ventaja que 
presenta, es que como la impregnación de las fibras no se lleva a cabo hasta que se consolida el material compuesto, el commingled se comporta como un preimpregnado flexible. La flexibilidad de estos pre-impregnados permite fabricar composites con geometrías complejas y garantiza una buena homogeneidad. La homogeneidad juega un papel importante ya que repercutirá en las propiedades mecánicas del perfil final ${ }^{38}$.
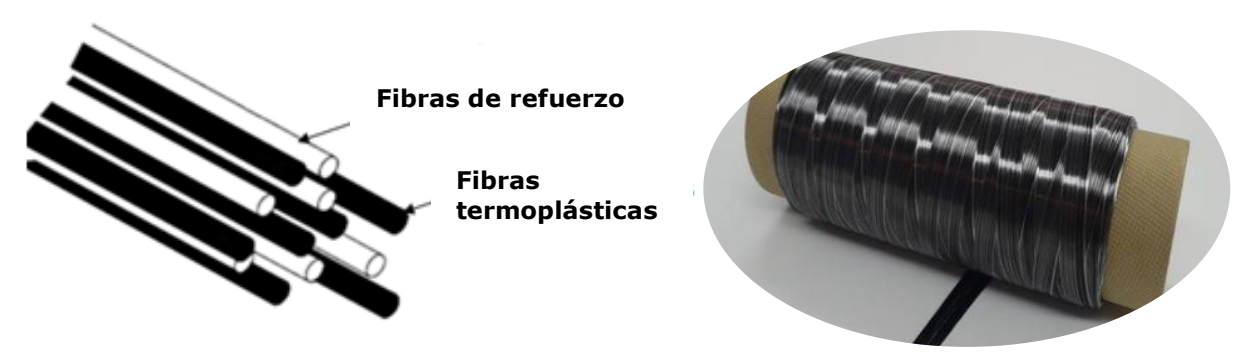

Figura 2.13 Esquema y fotografía de commingled

Como desventaja, debido a que los filamentos de la matriz no están embebidos en las fibras (impregnados), se requiere de un mayor esfuerzo para completar la consolidación, en comparación con los pre-impregnados ya pre-consolidados (como el tape). El principal inconveniente técnico de esta metodología de obtención de pre-impregnado, es que no todos los materiales poliméricos son fácilmente hilables.

Este procedimiento ha sido desarrollado recientemente para composites de PET virgen y fibra de vidrio ${ }^{39}$. Otra investigación muy reciente en este campo, es la presentada por Wiegand et al. ${ }^{40}$ donde estudiaron el comportamiento mecánico del commingled a partir filamentos de fibra de vidrio y filamentos de tres matrices poliméricas (polipropileno, poliamida y ácido poliláctico) usando silanos como agentes de acoplamiento entre las interfases. En relación con este trabajo de investigación, Chen et al. ${ }^{41}$ y otros autores ${ }^{42}$ han dado los primeros pasos en introducir materiales reciclados utilizando PET reciclado para obtener el hilo de refuerzo de los composites.

3) Por último, el towpreg, es un material pre-impregnado termoplástico que consiste en la adición del polímero en forma de polvo a las fibras de refuerzo (Figura 2.14). El proceso de obtención se lleva a cabo con la dispersión del material termoplástico en forma de pequeñas partículas de polvo sobre la fibra o refuerzo (roving). La adhesión entre ambos se genera gracias a las fuerzas electrostáticas y finalmente se impregna en una etapa posterior con aplicación de calor. Tras la impregnación, el towpreg se enfría y se bobina para usarlo con posterioridad en el proceso de pultrusión (consolidación). 


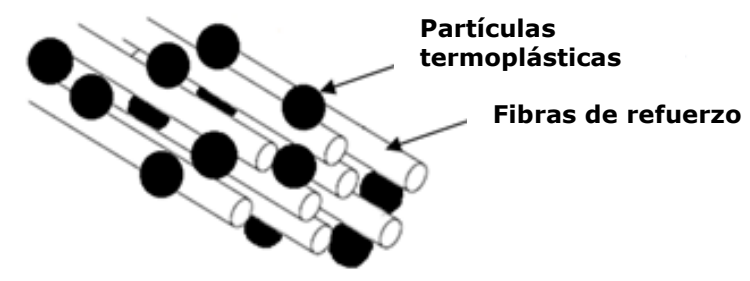

Figura 2.14 Esquema de towpreg: Fibras con polvos adheridos

Empleando este sistema, es posible extender el polvo a lo largo de todas las fibras, con lo que se logra un buen mezclado y, como consecuencia, el material puede ser calentado y enfriado rápidamente minimizando la degradación del polímero. Sin embargo, esta metodología cuenta con la dificultad de conseguir un tamaño de partícula suficientemente pequeño del material termoplástico (menor a 100 micras).

Novo et al. ${ }^{30}$ fueron los pioneros en el desarrollo de una línea de recubrimiento a partir de la matriz termoplástica en forma de polvo. Los materiales termoplásticos usados fueron grados comerciales amorfos de polipropileno con alto contenido de ramificaciones aromáticas. En sus trabajos se estudió el contenido de polvo incorporado en los towpregs en función de las condiciones de proceso (temperatura de horno, temperatura de consolidación y velocidad de tiro), como parámetro de medida de la efectividad del impregnado. El uso de estos semi-elaborados termoplásticos requirió la adaptación de los equipos convencionales de composites termoestables, para la fabricación del composite final. El bobinado de filamentos (filament winding), la pultrusión y el moldeo por compresión, fueron las tecnologías estudiadas como procesos de consolidación final ${ }^{30}$. Como aplicaciones, se elaboraron prototipos de recipientes a presión para gases y fluidos. Estos fueron sometidos a pruebas de explosión de presión interna. Otros autores emplearon este método de impregnación en polvo para la fabricación de laminados ("film stacking") a partir de poliamidas ${ }^{43}$.

La reciente evolución de los composites hacia la sustitución de las matrices termoestables por termoplásticas, ha permitido reconocer la gran importancia de estudiar y mejorar estos "materiales intermedios" para garantizar la buena impregnación de las fibras, debido a la alta viscosidad de los nuevos materiales introducidos. Como consecuencia, una mejora de la calidad del impregnado va a repercutir en el comportamiento mecánico del composite. 
Estos procesos de elaboración de pre-impregnados termoplásticos han mostrado las siguientes limitaciones ${ }^{15}$ :

- La variedad de combinaciones de materiales es limitada.

- El precio de los pre-impregnados.

- El procesamiento es difícil o limitado.

- La producción requiere mucha energía.

Las nuevas tendencias en el mercado relativas al proceso de pultrusión termoplástica, se centran en intentar compensar los inconvenientes antes mencionados, enfocadas principalmente, en conseguir aumentar la eficiencia del proceso y abaratar costes. En general, la eficiencia de una línea de pultrusión y las propiedades de la pieza resultante dependen del tipo de matriz, de la arquitectura de las fibras, de la estrategia usada para impregnar dichas fibras y de los parámetros de proceso (velocidad de tiro, temperatura del molde y postprocesado).

A diferencia de los trabajos anteriormente descritos, que se fundamentan en la utilización de nuevas técnicas, como la extrusión reactiva o la utilización de los materiales pre-impregnados para solventar el problema asociado a la viscosidad de los termoplásticos, esta investigación pretende ir un paso más allá, poniendo el foco en trabajar sobre la reología de las matrices termoplásticas con la finalidad de mejorar la impregnación y la adhesión (matriz termoplástica/fibra).

Adicionalmente, como se ha explicado anteriormente, este trabajo empleará como matriz de estudio los residuos de PET coloreados, contribuyendo así a la reducción de costes en cuanto a materia prima, así como a la disminución de la dependencia de materias primas vírgenes. Para conseguir una buena impregnación, y así el PET reciclado forme parte de un composite que presente buenas propiedades mecánicas, se han delimitado unos índices de viscosidad en los que debería moverse la matriz polimérica introducida (viscosidad en fundido, entre 25 y $100 \mathrm{~Pa} \cdot \mathrm{s}$ ). Estos límites han sido establecidos bajo el asesoramiento de empresas y universidades, que tienen una amplia experiencia en este sector de pultrusión termoplástica ${ }^{44}$. Para cumplir este objetivo será necesario diseñar métodos de modificación reológicos que permitan regular "a medida" la viscosidad de la matriz (mostrados en el Capítulo 3).

Adicionalmente, en este trabajo se ha diseñado una cámara de impregnación de alta presión en la cual se podrán estudiar la adhesión y el grado de impregnación de las morfologías poliméricas desarrolladas con las fibras de refuerzo. Las matrices 
investigadas serán integradas en los procesos de pultrusión termoplástica existentes, a partir del pre-impregnado con el material en forma de polvo (towpreg) y en forma de granza (tapes). La metodología de obtención a partir del commingled (matriz en forma de fibra) fue descartada debido a la imposibilidad técnica de obtener los materiales desarrollados en forma de filamentos o fibras.

Estas técnicas de obtención de perfiles presentan etapas muy distintas en sus líneas de procesado (principalmente porque en una el material es necesario que esté en forma de polvo y en otra en forma de granza o pellet). Es por este motivo que los parámetros de obtención y las características finales de los perfiles obtenidos son distintas, a pesar de partir de los mismos materiales.

\subsection{REFERENCIAS BIBLIOGRÁFICAS}

1. Welle Frank F-I. Guidance and criteria for safe recycling of post consumer polyethylene terephthalate (PET) into new food packaging applications. 2017:1-5. http://ilsi.eu/wp-content/uploads/sites/3/2017/12/PET-ILSI-Europe-Report-Update2017_interactif_FIN.pdf.

2. Park $\overline{\mathrm{SH}}$, Kim SH. Poly (ethylene terephthalate) recycling for high value added textiles. Fash Text. 2014;1(1):1-17. doi:10.1186/s40691-014-0001-x

3. Díaz LAQ. Diseño de una planta de reciclado de Tereftalato de polietileno (PET).

4. Plastics Insight. Polyethylene Terephthalate (PET): Production, Price, Market and its Properties. $\quad$ https://www.plasticsinsight.com/resin-intelligence/resinprices/polyethylene-terephthalate/. Published 2019.

5. Awaja F, Pavel D. Recycling of PET. Eur Polym J. 2005;41(7):1453-1477. doi:10.1016/j.eurpolymj.2005.02.005

6. Dutt K, Soni RK. A review on synthesis of value added products from polyethylene terephthalate (PET) waste. Polym Sci - Ser B. 2013;55(7-8):430-452. doi: 10.1134/S1560090413070075

7. Andrés EV. Uso Del PET Reciclado Para Envase Alimentario. Informe de Novedades Tecnológicas.; 2010.

8. Petcore; Plastics recyclers europe; European federation of bottle waters. PET MARKET IN EUROPE STATE OF PLAY. 2020.

9. Petcore Europe. https://www.petcore-europe.org/.

10. Awaja F, Pavel D. Recycling of PET. Eur Polym J. 2005;41(7):1453-1477. doi:10.1016/j.eurpolymj.2005.02.005

11. Al-Sabagh AM, Yehia FZ, Eshaq G, Rabie AM, EIMetwally AE. Greener routes for recycling of polyethylene terephthalate. Egypt $J$ Pet. 2016;25(1):53-64. doi:10.1016/j.ejpe.2015.03.001

12. Tavares AA, Silva DFA, Lima PS, Andrade DLACS, Silva SML, Canedo EL. Chain extension of virgin and recycled polyethylene terephthalate. Polym Test. 2016;50:2632. doi: $10.1016 / \mathrm{j}$.polymertesting.2015.11.020

13. Daver F, Gupta R, Kosior E. Rheological characterisation of recycled poly(ethylene terephthalate) modified by reactive extrusion. J Mater Process Technol. 2008;204(13):397-402. doi:10.1016/j.jmatprotec.2007.11.090

14. Miravete A. Materiales compuestos. Temes de disseny. 2002;(20):123-139.

15. Vaidya UK, Chawla KK. Processing of fibre reinforced thermoplastic composites. Int Mater Rev. 2008;53(4):185-218. doi:10.1179/174328008X325223

16. Liu $Y$, Farnsworth $M$, Tiwari A. A review of optimisation techniques used in the composite recycling area: State-of-the-art and steps towards a research agenda. J Clean Prod. 2017;140:1775-1781. doi:10.1016/j.jclepro.2016.08.038

17. Kinvi-Dossou G, Matadi Boumbimba $\mathrm{R}$, Bonfoh N, et al. Innovative acrylic thermoplastic composites versus conventional composites: Improving the impact performances. Compos Struct. 2019;217(December 2018):1-13. 
doi:10.1016/j.compstruct.2019.02.090

18. Akca E, Gursel A. A Review on the Matrix Toughness of Thermoplastic Materials. Period Eng Nat Sci. 2015;3(2):1-8. doi:10.21533/pen.v3i2.52

19. OWENS CORNING COMPOSITE MATERIALS L. VISIÓN DEL MERCADO PARA COMPOSITE SOLUTIONS. PUb No 10018798. 2013.

20. Association EPT. World Pultrusion Conference 2018. 2018.

21. Mitschang P, Christmann M. Continuous Fiber Reinforced Profiles in Polymer Matrix Composites. Woodhead Publishing Limited; 2012. doi: $10.1533 / 9780857096258.2 .209$

22. Eiffel G. El presente informe es el resultado del proyecto financiado por IMPIVA a través del Programa de Fomento de la Innovación en Institutos Tecnológicos , 2010.

23. Miazza NL. Principales Avances En Pultrusión y SMC.; 2010.

24. Gazechim Ibérica. https://www.gazechim.es/procesos-de-aplicacion/pultrusion/.

25. Van De Velde K, Kiekens P. Thermoplastic pultrusion of natural fibre reinforced composites. Compos Struct. 2001;54(2-3):355-360. doi:10.1016/S02638223(01)00110-6

26. Velde $\mathrm{K}$ Van De, Kiekens $\mathrm{P}$, Mayer $\mathrm{C}$, et al. Pultrusion of unidirectional composites with thermoplastic matrices. Compos Part A Appl Sci Manuf. 2015;54(3):191-196. doi:10.1016/S0263-8223(01)00110-6

27. Epple S, Bonten C. Production of continuous fiber thermoplastic composites by in-situ pultrusion. AIP Conf Proc. 2014;1593(February 2015):454-457. doi: $10.1063 / 1.4873820$

28. Chen K, Jia M, Hua S, Xue P. Optimization of initiator and activator for reactive thermoplastic pultrusion. J Polym Res. 2019;26(2). doi:10.1007/s10965-019-1708-6

29. Luisier A, Bourban PE, Månson JAE. Reaction injection pultrusion of PA12 composites: Process and modelling. Compos Part A Appl Sci Manuf. 2003;34(7):583-595. doi:10.1016/S1359-835X(03)00101-5

30. Novo PJ, Silva JF, Nunes JP, Marques AT. Pultrusion of fibre reinforced thermoplastic pre-impregnated materials. Compos Part B Eng. 2016;89:328-339. doi:10.1016/j.compositesb.2015.12.026

31. Novo PJ, Silva JF, Nunes JP, Marques AT. Advances in thermoplastic pultruded composites. ICCM Int Conf Compos Mater. 2015;2015-July(July):19-24.

32. Schäfer J, Gries T. Braiding pultrusion of thermoplastic composites. Adv Braid Technol. 2016:405-428. doi:10.1016/b978-0-08-100407-4.00017-x

33. Miller AH, Dodds N, Hale JM, Gibson AG. High speed pultrusion of thermoplastic matrix composites. Compos Part A Appl Sci Manuf. 1998;29(7):773-782. doi: 10.1016/S1359-835X(98)00006-2

34. McGregor OPL, Somashekar AA, Bhattacharyya D, McGregor OPL, Duhovic M. Preimpregnated natural fibre-thermoplastic composite tape manufacture using a novel process. Compos Part A Appl Sci Manuf. 2017;101:59-71. doi:10.1016/j.compositesa.2017.05.025

35. Novo PJ, Silva JF, Nunes JP, Van Hattum FWJ, Marques AT. Development of a new pultrusion equipment to manufacture thermoplastic matrix composite profiles. ECCM 2012 - Compos Venice, Proc 15th Eur Conf Compos Mater. 2012;(June):18-25.

36. Marques AT, Azurem C De. PROCESSING OF CARBON REINFORCED THERMOPLASTIC PRE- IMPREGNATED MATERIALS. ECCM17 - 17th European Conference on Composite Materials Munich, Germany, 26-30th; 2016.

37. Santulli C, Gil RG, Long AC, Clifford MJ. Void content measurements in commingled E-glass/polypropylene composites using image analysis from optical micrographs. Sci Eng Compos Mater. 2002;10(2):77-90. doi:10.1515/secm.2002.10.2.77

38. Alagirusamy R, Ogale V. Development and characterization of GF/PET, GF/nylon, and GF/PP commingled yarns for thermoplastic composites. J Thermoplast Compos Mater. 2005;18(3):269-285. doi:10.1177/0892705705049557

39. Tomić NZ, Vuksanović M, Međo $B$, et al. Optimizing the thermal gradient and the pulling speed in a thermoplastic pultrusion process of PET/E glass fibers using finite element method. Metall Mater Eng. 2018;24(2):103-112. doi:10.30544/367

40. Wiegand N, Mäder E. Commingled yarn spinning for thermoplastic/glass fiber composites. Fibers. 2017;5(3). doi:10.3390/fib5030026

41. Chen JC, Wu CM, Pu FC, Chiu CH. Fabrication and mechanical properties of selfreinforced poly(ethylene terephthalate) composites. Express Polym Lett. 
2011;5(3):228-237. doi:10.3144/expresspolymlett.2011.22

42. M. Evstatiev, I. Angelov KF. Structure and Properties of Microfibrillar-Reinforced Composites Based on Thermoplastic PET/LDPE Blends After Manufacturing by Means of Pultrusion. 20AD. doi:10.1002/pen.21538

43. Mayer C, Wang X, Neitzel M, Gmbh V. Macro- and micro-impregnation phenomena in continuous manufacturing of fabric reinforced. Compos Part A Appl Sci Manuf. 1998;29(7):783-793. doi:https://doi.org/10.1016/S1359-835X(98)00056-6

44. CiComp Competence in composites. https://www.circomp.de/home.html. 


\section{Ca pítulo 3}

En este capítulo, se presentan, en primer lugar, las técnicas experimentales con las que se han llevado a cabo las modificaciones reológicas de los materiales residuales estudiados, así como las técnicas de caracterización micro y macro-estructurales empleadas para el estudio de estos materiales modificados. Posteriormente, se presenta el diseño y la puesta a punto de la cámara de impregnación utilizada para la validación del proceso de impregnación de los materiales desarrollados en granza. Por último, se describen, de forma experimental, los dos procesos de pultrusión utilizados para la obtención de perfiles finales (pultrusión en fundido y pultrusión en polvo) y las técnicas utilizadas para la caracterización y validación de los mismos.

\section{Técnicas Experimentales: modificación reológica, metodología de} procesado y caracterización de composites termoplásticos

Como se ha descrito anteriormente, esta investigación nace de la preocupación por reciclar los residuos de PET menos valorizados mediante su introducción en procesos de producción de alto valor y demanda como materia prima secundaria. La introducción de estos termoplásticos en procesos de pultrusión, requiere que sean modificados reológicamente para cumplir con los parámetros de viscosidad establecidos. En este capítulo se van a exponer las técnicas experimentales con las que se lleva a cabo la modificación reológica (sección 3.1) y las técnicas de análisis empleadas para la caracterización de los materiales obtenidos por estas modificaciones (sección 3.2).

Una vez estudiados los métodos de modificación reológica de los materiales residuales a utilizar, en la sección 3.3 se muestra cómo se llevó a cabo el diseño y puesta a punto de una cámara de impregnación, empleada con el fin de estudiar y validar la impregnación de las fibras con los materiales modificados reológicamente. Una vez se verificó la calidad de la impregnación de las fibras con los diferentes polímeros residuales modificados (caracterización de los semi-elaborados), se hizo una selección de los mismos para validar estos materiales en la fabricación de un perfil final. La validación se llevó a cabo por la metodología de consolidación conocida como pultrusión termoplástica. Esta técnica fue aplicada para los materiales obtenidos en granza y a partir del material en polvo.

El primer proceso que se muestra en este capítulo es la pultrusión en fundido (sección 3.4). Cabe destacar que esta metodología de obtención es un proceso actualmente industrializado, en el que se utilizan las matrices en forma de granza, lo cual permite utilizar los materiales desarrollados de una forma directa (según 
salen de la extrusión). El proceso de obtención de los perfiles se realizó en colaboración con la empresa alemana propietaria de esta tecnología (Circomp).

El segundo proceso que se utilizó para la obtención de perfiles es la pultrusión en polvo (sección 3.5), en la cual se introducen las matrices en forma de polvo. Como desventaja, incluye el paso del material de granza a polvo, sin embargo, al presentar este pequeño tamaño se consigue una excelente impregnación, por el aumento importante de área superficial entre la matriz y las fibras. Esta metodología fue empleada durante la estancia realizada en la universidad de ISEP en Oporto (Portugal).

Finalmente, en la sección 3.6 se muestran las técnicas de caracterización de los perfiles compuestos, obtenidos por ambos métodos de pultrusión.

\subsection{TÉCNICAS DE MODIFICACIÓN REOLÓGICA}

La modificación reológica de las matrices (que se desarrollará en el Capítulo 4) se llevó a cabo en fundido, en una línea de extrusión. La extrusión es uno de los métodos de procesado de plásticos más utilizados. Se trata de un proceso en continuo y su empleo está ampliamente extendido tanto para la obtención de mezclas poliméricas como para modificación de polímeros mediante aditivos (extrusión reactiva).

Este proceso se ha llevado a cabo empleando una extrusora Leistritz 27 de doble husillo con una relación L/D de 36 (Figura 3.1). La alimentación de los polímeros (mezclado físico) y del aditivo (mezclado químico) se han dosificado desde un puerto lateral el cual dispone de su propio husillo.

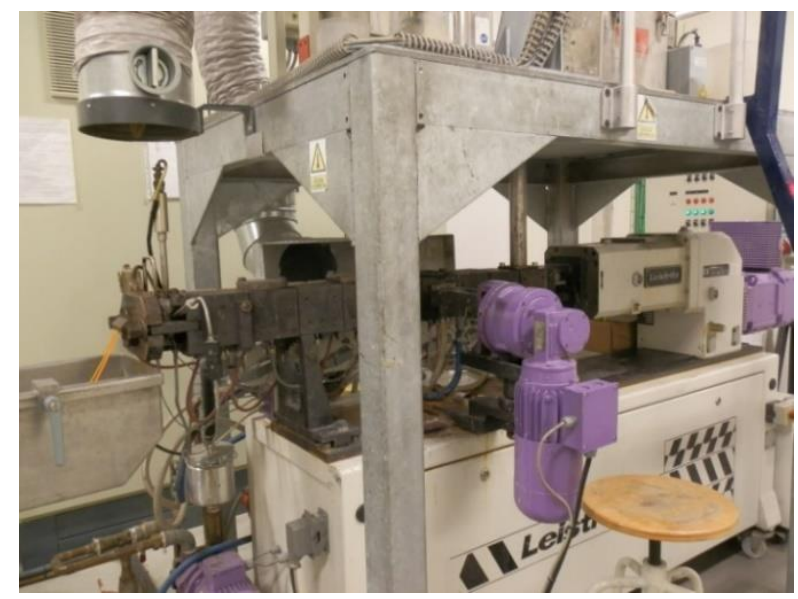

Figura 3.1 Extrusora corrotante de doble husillo empleada para la modificación de los materiales 
Para asegurar el tratamiento reológico de los materiales residuales, es necesario controlar los cambios físicos que experimenta el PET durante su procesado por extrusión. Por este motivo (previamente a la realización de los tratamientos reológicos) se llevará a cabo un diseño de experimentos (DOE) en el que se estudiará el efecto de los siguientes factores sobre la degradación del PET ${ }^{6}$ :

- La temperatura de proceso: por un lado, el empleo de altas temperaturas puede producir degradaciones térmicas del polímero $y$, por otro lado, bajas temperaturas hacen que el material presente una elevada viscosidad que se traduce en altas presiones en el interior de la extrusora. Debe encontrarse una temperatura de compromiso en la que el PET funda y presente viscosidades bajas sin que llegue a un estado de degradación que lo invalide.

- La velocidad de rotación o velocidad de husillo: se mide en revoluciones por minuto y debe alcanzarse un valor de compromiso, pues tiene dos efectos contrapuestos. Por un lado, el empleo de altas velocidades favorece el efecto cizalla que facilita la escisión de las cadenas del PET. Sin embargo, la velocidad de rotación está relacionada con el tiempo de residencia dentro de la extrusora. En este sentido, bajas velocidades implican tiempo de ciclo mayor y, por consiguiente, mayor tiempo de permanencia del PET en la extrusora.

- El caudal de alimentación: este parámetro define la cantidad de material obtenido por unidad de tiempo. Es importante remarcar que el caudal seleccionado debe estar en concordancia con la velocidad de rotación, es decir, la cantidad de material que se dosifica no puede superar la capacidad del husillo.

- La geometría del husillo: la configuración de las distintas zonas del husillo determina la cizalla que se le aplica al PET, influyendo en la viscosidad del polímero. La configuración empleada para este trabajo (Figura 3.2) se compone de varias zonas de amasamiento o cizalla (KB), así como de arrastre o antirretorno (GFA).

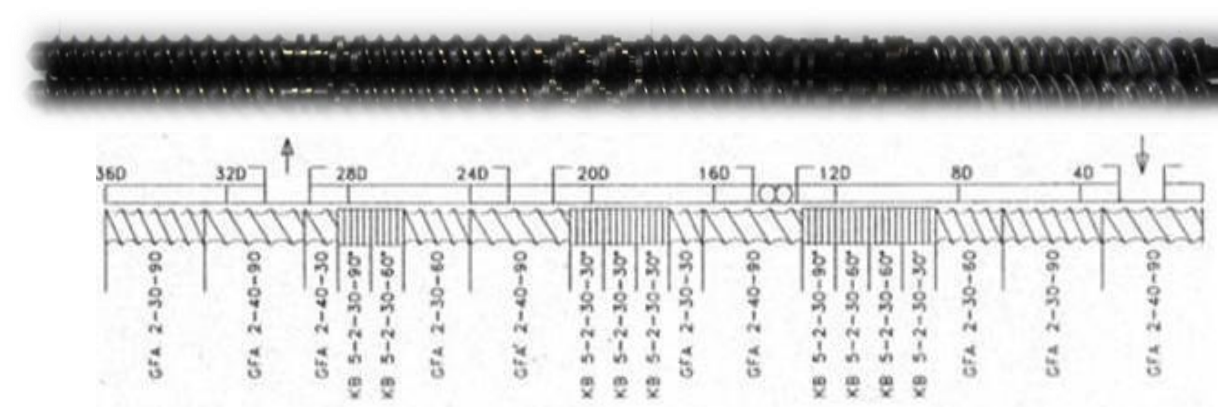

Figura 3.2 Configuración del husillo empleado para el tratamiento de los materiales 
En la Tabla 3.1 se muestran los factores y niveles seleccionados para la realización del DOE, con tres factores (velocidad de husillo, temperatura y caudal) a dos niveles (con el nivel más alto y el más bajo posible).

Tabla 3.1 Factores y niveles del proceso de extrusión

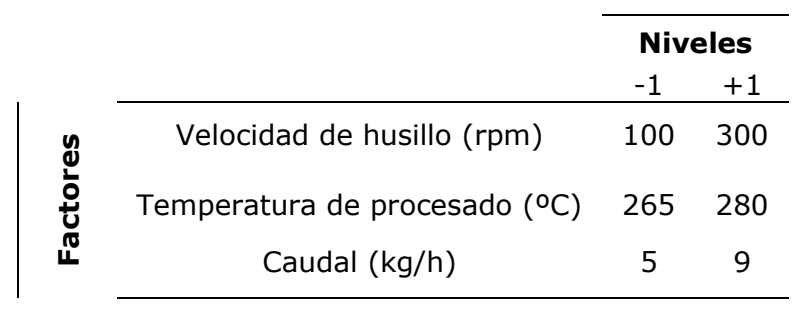

Una vez realizados los tratamientos reológicos en la línea de extrusión, es necesario utilizar el proceso de inyección para poder moldear los materiales desarrollados (obtenidos en forma de granza) en probetas y así caracterizarlos mecánicamente.

La inyección es el proceso más popular para el moldeo de materiales termoplásticos. Se trata de un proceso semicontinuo que consiste en la inyección del polímero en fundido a altas presiones en el interior de un molde cerrado y atemperado. Una vez en el interior del molde, el polímero solidifica y la pieza final se obtiene al abrir el molde y retirarla de la cavidad.

La inyectora que se utilizó en esta investigación fue una Krauss Maffei modelo KM 200 (Figura 3.3). Se obtuvieron probetas tipo 1A, de acuerdo a la norma ISO 3167. Estas probetas se utilizan directamente para los ensayos a tracción, pero también se pueden cortar a las dimensiones requeridas para los ensayos de impacto Charpy.

Las temperaturas de inyección y de molde seleccionadas fueron las recomendadas por los fabricantes de PET. El resto de los parámetros, como la velocidad de inyección, velocidad de cizalla, presión posterior, tiempo de presión posterior y tiempo de enfriamiento fueron optimizados en estudios previos del grupo de investigación. El perfil de temperatura de los cilindros fue de 240 a $270^{\circ} \mathrm{C}$ y la temperatura del molde fue de $50^{\circ} \mathrm{C}$. 


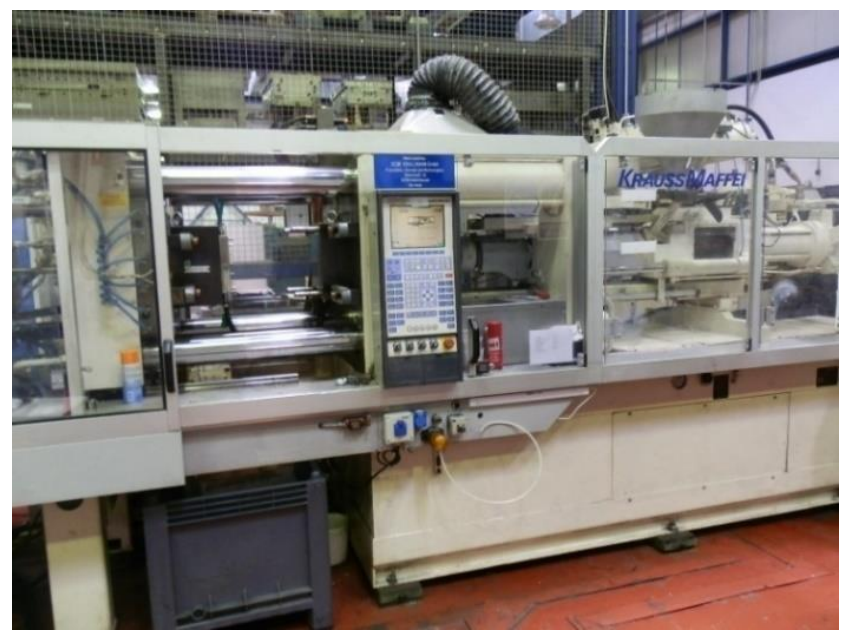

Figura 3.3 Máquina de inyección empleada para la obtención de probetas

\subsection{TÉCNICAS DE CARACTERIZACIÓN DE LOS MATERIALES MODIFICADOS}

\subsubsection{Análisis térmico}

Bajo la denominación de análisis térmico se engloban un conjunto de técnicas analíticas que estudian cambios físicos o químicos de una muestra, cuando es sometida a un proceso programado de temperatura en atmósfera controlada. En todos los casos, el objetivo es establecer una relación entre la temperatura y las propiedades físicas o químicas del material. La instrumentalización de estos ensayos permite obtener una serie de curvas, a partir de las cuales se pueden discernir los cambios térmicos que se producen en la muestra.

\section{Calorimetría diferencial de barrido (DSC)}

La calorimetría diferencial de barrido es una técnica cuantitativa que consiste en el calentamiento simultáneo y programado de una muestra y una referencia. El instrumento es capaz de detectar las diferencias entre el flujo de calor de la muestra y la referencia. El principio básico de esta técnica es que, cuando la muestra experimenta una transformación física, necesita más o menos calor que la referencia para mantener la misma temperatura.

Los resultados de los experimentos se recogen en curvas de flujo calorífico en función de la temperatura o del tiempo. Todas las diferencias entre el calor necesario para llevar a cabo el programa de temperatura en la muestra y la referencia se registran y dan lugar a picos que pueden ser utilizados para calcular las entalpías asociadas a las transiciones. 
Las temperaturas de fusión $\left(T_{f}\right)$, transición vítrea $\left(T_{g}\right)$ y cristalización $\left(T_{c}\right)$, así como la cristalinidad $(\mathrm{X})$ de las muestras se midieron por calorimetría diferencial de barrido (DSC), con un equipo Mettler Toledo DSC 851e (Figura 3.4). El rango de temperaturas fue de 25 a $300^{\circ} \mathrm{C}$ y la velocidad de calentamiento de $20^{\circ} \mathrm{C} / \mathrm{min}$ bajo flujo de nitrógeno. Todos los materiales fueron sometidos a un calentamiento por encima de su punto de fusión para eliminar el historial térmico. La entalpía seleccionada de un PET completamente cristalino fue de $140 \mathrm{~J} / \mathrm{g}^{7,8}$.

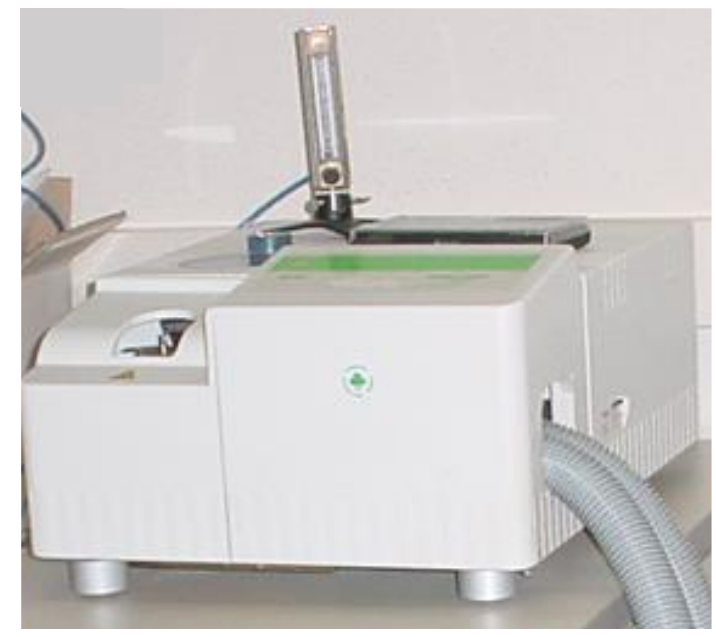

Figura 3.4 Equipo de DSC empleado para la caracterización térmica de las muestras de PET

\subsubsection{Comportamiento mecánico}

Los ensayos mecánicos describen el comportamiento de los materiales cuando son sometidos a una fuerza externa. En este trabajo se han realizado ensayos de tracción y medidas de resistencia al impacto Charpy.

\section{Propiedades mecánicas a tracción}

Los ensayos a tracción, en un sentido amplio, son una medida de la capacidad del material para soportar fuerzas que lo estiran y deforman. Las máquinas para realizar estos ensayos constan de un brazo fijo y otro móvil, ambos dotados de mordazas donde se fijan los extremos de las probetas. Una vez que comienza el ensayo, el brazo móvil se desplaza a una velocidad constante preestablecida. El desplazamiento del brazo genera una deformación y una carga sobre la probeta que son recogidas por el equipo. A partir de los datos recogidos durante el ensayo, se crean las curvas de esfuerzo-deformación, de las cuales se obtienen los Módulos de Young. 
Para la realización de estos ensayos las muestras de PET obtenidas en el Capítulo 4 fueron moldeadas mediante el proceso de inyección. Se utilizó una máquina universal de ensayos Instron modelo 5500R60025 (Figura 3.5). El Módulo de Young y la Resistencia a tracción o Carga de rotura se midieron a temperatura ambiente a una velocidad de $1 \mathrm{~mm} \cdot \mathrm{min}^{-1}$ y $10 \mathrm{~mm} \cdot \mathrm{min}^{-1}$ respectivamente, de acuerdo con la norma ISO 527-1. Para cada material se analizaron cinco muestras para la obtención de valores medios. Antes del ensayo, las probetas fueron acondicionadas durante 24 horas en una cámara a $23^{\circ} \mathrm{C}$ y una humedad relativa del $50 \%$.
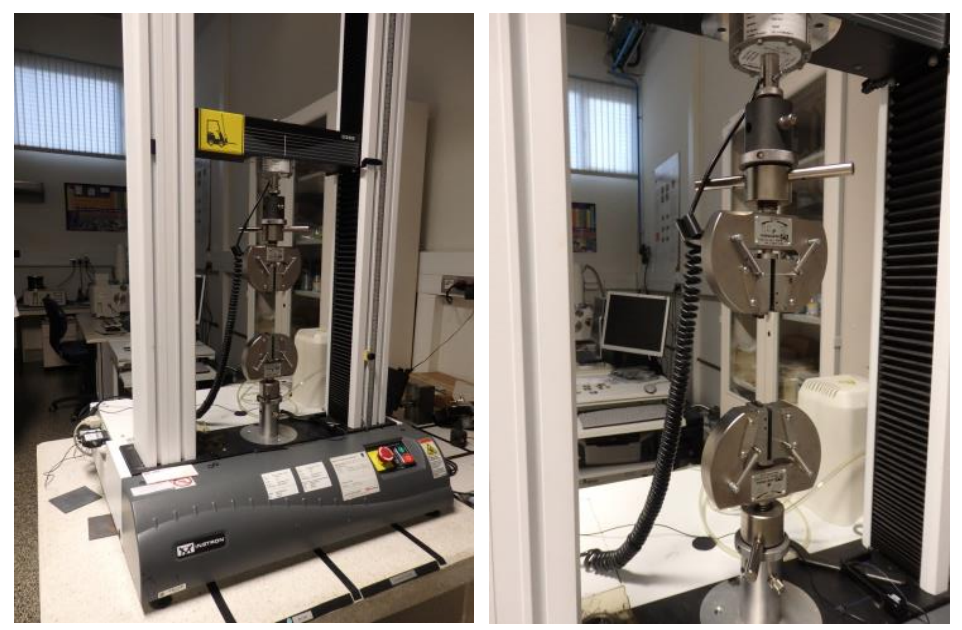

Figura 3.5 Máquina universal de ensayos Instron

\section{Impacto Charpy}

La resistencia al impacto se define como la energía absorbida por unidad de área del material durante un ensayo de impacto. La resistencia al impacto no es una propiedad intrínseca del material, sino que depende de otros factores como pueden ser la temperatura, la humedad, la velocidad de impacto o la dirección del impacto.

Para medir la resistencia al impacto se eligió el método Charpy. Esta técnica mide la energía necesaria para romper la probeta, la cual es la suma de energías para flexionar la probeta, iniciar la fractura, propagarla y acelerar la parte de la probeta que sale despedida tras el ensayo. La energía perdida por fricción y vibración del aparato es mínima, por lo que se consideran despreciables. 
Los ensayos de impacto realizados en esta investigación se llevaron a cabo de acuerdo a la norma ISO 179. Se cortaron las cabezas de las probetas de inyección para ajustar la parte central a prismas de $80 \mathrm{~mm}$ y a continuación se las realizó una entalla de $2 \mathrm{~mm}$ en la parte central. Una vez que se fijan en el impactor, el martillo golpea la probeta y registra la diferencia de energía potencial entre el punto inicial y final para devolver la energía consumida durante el ensayo. La resistencia al impacto de esa probeta vendrá expresada como el cociente de la energía devuelta por el equipo y la sección de la probeta. Para cada referencia, se realizaron seis impactos y las probetas fueron acondicionadas durante 24 horas a $23^{\circ} \mathrm{C}$ y una humedad relativa del $50 \%$. Todos los ensayos fueron realizados en péndulo de impacto modelo CEAST Resil 6957 que se muestra en la Figura 3.6.

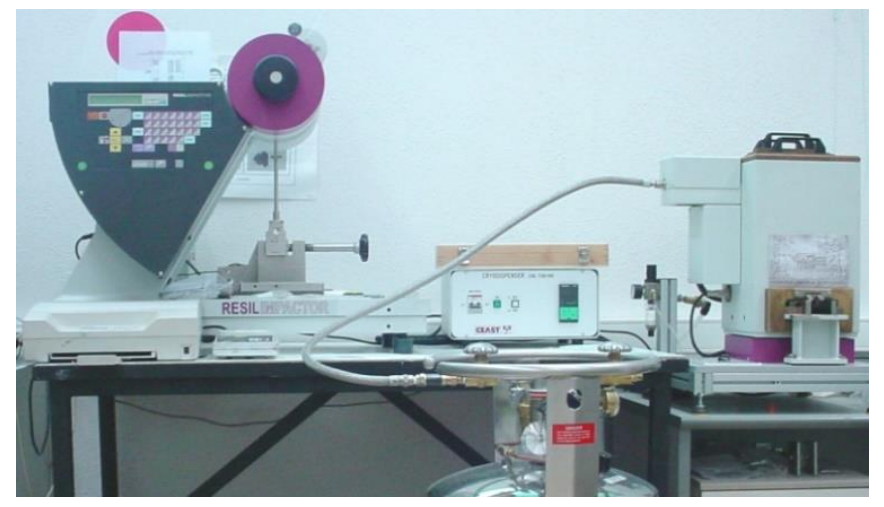

Figura 3.6 Péndulo empleado para los ensayos de impacto Charpy con entalla

\subsubsection{Medidas de viscosidad}

Las propiedades reológicas de los materiales poliméricos miden su resistencia a la fluencia y a la deformación. El análisis reológico de los materiales nos proporciona una información esencial sobre su procesabilidad. La viscosidad de un polímero está estrechamente relacionada con el peso molecular, y éste guarda una relación directa con sus propiedades. Por estas razones, en esta investigación se han realizado ensayos de viscosidad sobre polímero fundido, como el índice de fluidez o la reometría dinámica, y también en disolución.

Viscosidad en fundido: Índice de fluidez (MFI) y reometría dinámica

El índice de fluidez es un análisis reológico que permite conocer, de forma sencilla, la fluidez de un polímero. Se define como la cantidad de material que fluye a través de un orificio capilar en 10 minutos a una presión y temperatura constante. 
El ensayo consiste en la introducción y compactación de la granza del material en el interior de un barril calefactado por encima de la temperatura de fusión del polímero. A continuación, la masa fundida es empujada a través del barril hasta el orificio de salida por acción de un pistón conectado a una masa. Los resultados se expresan en g/10min, es decir, la cantidad de material que es capaz de fluir a través del agujero en diez minutos.

El carácter altamente higroscópico junto a la degradación térmica del PET, hace que la técnica de medición del Índice de Fluidez (MFI) esté contraindicada, prefiriéndose trabajar con el parámetro de Índice de viscosidad intrínseca $[\mu]$ determinada por viscosimetría en solución. No obstante, desde el punto de vista tecnológico, la correlación entre dicho parámetro y las condiciones de procesamiento en fundido no es tan sencilla o directa, careciendo muchas veces de sentido práctico en el establecimiento de parámetros de procesado ${ }^{9}$.

Basados en las evidencias bibliográficas encontradas sobre el uso del MFI ${ }^{10,11}$, se procedió a la determinación del MFI en condiciones controladas de humedad y temperatura con el fin de minimizar los riesgos de degradación hidrolítica. Todas las muestras fueron acondicionadas a $110^{\circ} \mathrm{C}$ en una deshumidificadora. La determinación del índice de fluidez de los materiales ha sido realizada en un equipo marca CEAST modelo 7026 a una temperatura de $260^{\circ} \mathrm{C}$ y con una masa de $1 \mathrm{Kg}$ (Figura 3.7).

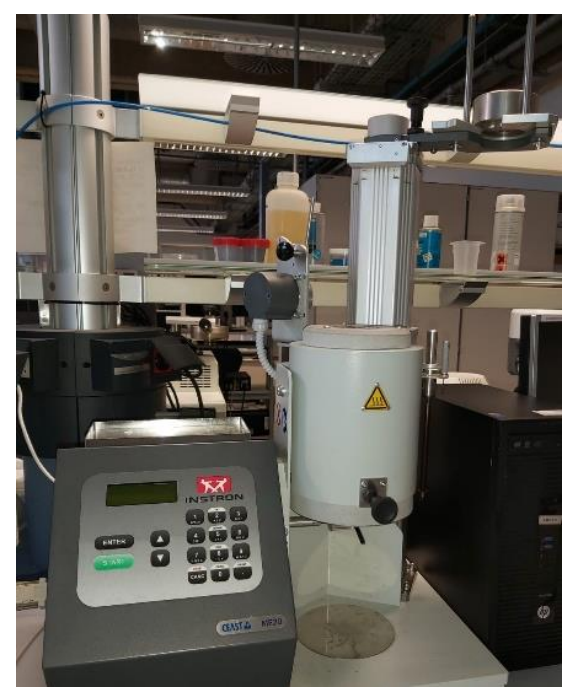

Figura 3.7 Equipo de MFI empleado en la caracterización reológica 


\section{Reometría dinámica}

La reometría dinámica es uno de los ensayos más importantes para optimizar el procesado de los materiales, ya que nos proporciona información reológica de los materiales desarrollados en un amplio rango de temperaturas, permitiéndonos regular las frecuencias y el tiempo del ensayo. La realización del ensayo dinámico a bajas frecuencias $(1 \mathrm{~Hz})$, permite simular las condiciones de proceso que experimenta el PET durante la pultrusión.

La reometría dinámica o viscosimetría rotacional consta básicamente de dos partes que se encuentran separadas por el fluido a estudiar. El movimiento de una de estas partes provoca la aparición de un gradiente de velocidades a lo largo del fluido. Para determinar la viscosidad del fluido se mide el esfuerzo necesario para producir una determinada velocidad angular.

El equipo que se utilizó para realizar las mediciones de viscosidad en estado fundido fue un reómetro dinámico HAAKE Rheostress 600 (Figura 3.8). Se utilizó la geometría placa-placa $(20 \mathrm{~mm}$ ) y un espacio entre placas de $1.6 \mathrm{~mm}$. En este caso, el fluido se encuentra entre dos placas paralelas, la superior gira y la inferior permanece inmóvil. La viscosidad compleja $\left(\mu^{*}\right)$ se midió a una frecuencia de $1 \mathrm{~Hz}$ variando la temperatura desde $265^{\circ} \mathrm{C}$ a $300^{\circ} \mathrm{C}$ para conocer un amplio comportamiento de la viscosidad con la temperatura. Para evitar posibles cambios de masa molar durante la preparación de la muestra, las matrices de PET se depositaron directamente en el reómetro precalentado.

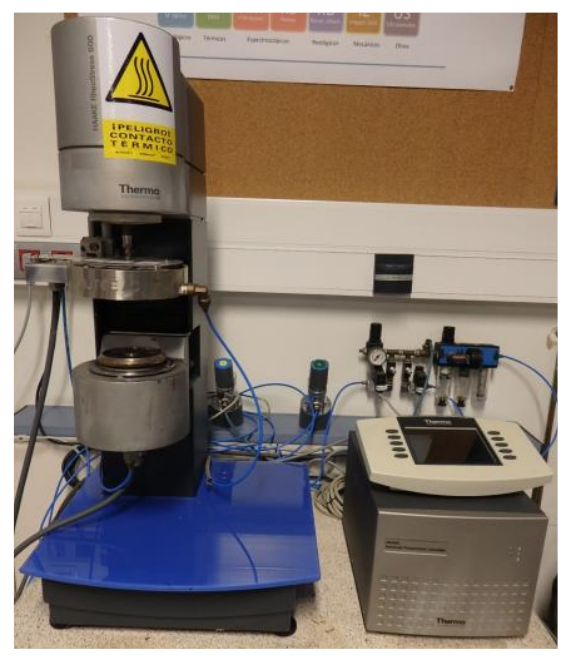

Figura 3.8 Reómetro dinámico utilizado en la caracterización reológica 


\section{Viscosidad en disolución: viscosidad intrínseca}

La viscosidad intrínseca $[\mu$ ] representa una medida del peso molecular promedio. Esta técnica de análisis es ampliamente utilizada en para asignar un valor de viscosidad relativa a los diversos grados y aplicaciones de PET, ya que, permite asegurar el valor preciso de viscosidad, sin que esta se vea modificada por otros parámetros como la temperatura o la cizalla (métodos de análisis viscosidad en fundido).

Los ensayos de viscosidad en disolución consistieron en la disolución de las muestras en una solución de fenol y 1,1,2,2-tetracloroetano (60/40 \% en peso) a seis niveles de concentración, desde $0.2 \mathrm{~g} / \mathrm{dl}$ hasta $1 \mathrm{~g} / \mathrm{dl}$. A continuación, cada nivel se carga en un viscosímetro Ubbelhode que se introduce en un baño de agua a $30^{\circ} \mathrm{C}$. Una vez estabilizada la temperatura, con ayuda de un pipeteador se carga el bulbo superior y se mide el tiempo necesario para recorrer la distancia entre las marcas inicial y final del viscosímetro. Cada nivel de concentración supone un punto de las rectas de la viscosidad inherente y reducida a partir de las cuales se puede obtener la viscosidad intrínseca ( $\mathrm{dl} / \mathrm{g}$ ) de acuerdo a la norma ASTM D4603.

Los valores de viscosidad intrínseca $[\mu]$ se pueden relacionar con el peso molecular viscosimétrico (Mv) mediante la siguiente ecuación de MarkHouwink ${ }^{12,13}$. La viscosidad intrínseca $[\mu]$ es una medida del peso molecular de los polímeros y, por lo tanto, refleja el punto de fusión del material, la cristalinidad y la resistencia a la tracción.

$$
[\mu]=K M^{a}
$$

Para un uso correcto de esta expresión, es necesario tener los valores de $\mathrm{K}$ y a, que son específicos para las condiciones fijas de tipo de polímero, disolvente y temperatura. Para la condición del PET disuelto en 60/40 Fenol-1,12,2tetracloroetano, se pueden encontrar las siguientes relaciones en la literatura ${ }^{12,13}$.

$$
\begin{aligned}
& {[\mu]=3.72 \times 10^{-4}(M n)^{0.73}} \\
& {[\mu]=4.68 \times 10^{-4}(M w)^{0.68}}
\end{aligned}
$$

Esta relación se ha empleado en el Capítulo 4 para obtener los pesos moleculares de las muestras de PET desarrolladas a partir de los diferentes tratamientos. La longitud de la cadena de polímero en PET determina el peso molecular del material y con él las propiedades físicas. 


\subsubsection{Métodos espectroscópicos}

Los métodos espectroscópicos agrupan un conjunto de técnicas instrumentales que se basan en el estudio de la interacción entre la radiación electromagnética y la materia. En este trabajo de investigación se ha utilizado la técnica de espectroscopía de infrarrojo por transformada de Fourier (FTIR).

\section{Espectroscopía de infrarrojo (FTIR)}

Esta técnica consiste en irradiar las muestras con frecuencias del infrarrojo para producir vibraciones moleculares. Estas vibraciones son registradas por el equipo para dar lugar a bandas en los espectros de infrarrojo. Mediante esta técnica es posible determinar, de forma cualitativa, los enlaces presentes en la muestra, ya que las bandas asociadas a las vibraciones son características de cada enlace. EI FTIR dispone de un accesorio ATR (Reflectancia Total Atenuada) que posibilita trabajar por reflexión con pequeñas cantidades de muestra, lo cual permite analizar de una manera directa la muestra en estado sólido sin necesidad de someterla a una preparación previa.

En este trabajo de investigación, el FTIR se ha empleado para comprobar la procedencia polimérica de los materiales residuales, así como para evaluar los cambios estructurales del PET con las modificaciones reológicas. Los ensayos se Ilevaron a cabo en el equipo Bruker Tensor 27 con una resolución de $4 \mathrm{~cm}^{-1}$ y 16 escaneos en la región de 500 a $4000 \mathrm{~cm}^{-1}$ (Figura 3.9).

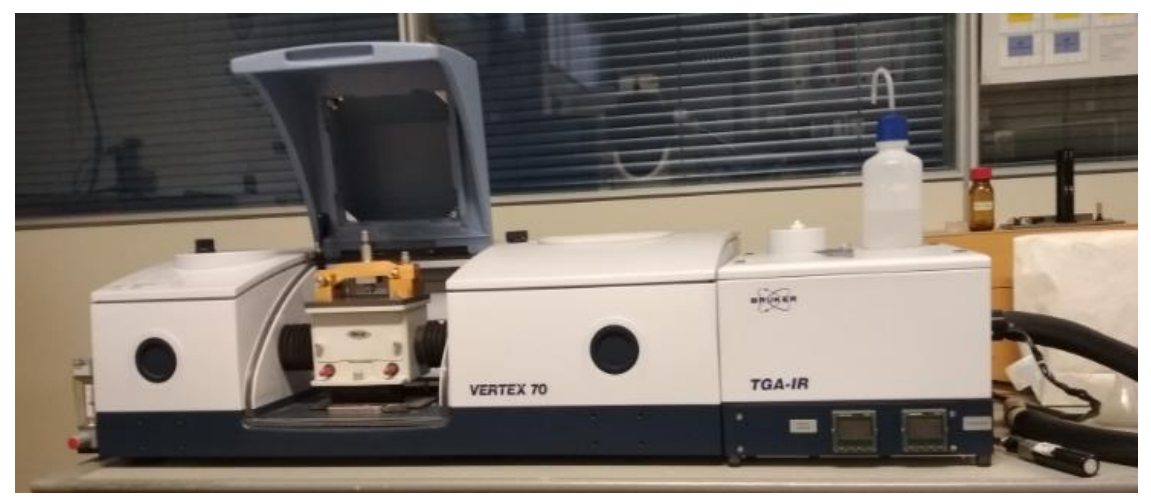

Figura 3.9 Equipo de FTIR utilizado 


\subsubsection{Otras técnicas instrumentales de caracterización}

\section{Cromatografía de permeación en gel (GPC)}

La determinación de pesos moleculares se llevó a cabo mediante el uso de un cromatógrafo de líquidos de alta resolución con un detector de fotodiodos (HPLCPDA) empleando una columna de permeación de gel (GPC). Para la calibración se han utilizado patrones de polimetilmetacrilato (PMMA) de pesos moleculares conocidos: $675.500,265.300,146.500,72.000,31.110,9.150,4.770,1.840$ y 885 $\mathrm{g} / \mathrm{mol}$. El equipo utilizado fue de Waters 1515 equipado con Waters 717 plus autosampler y un detector Waters 996 PDA con el software Empower 3. El cálculo de la distribución de pesos moleculares de las muestras se llevó a cabo mediante el software de Waters.

Para el análisis, se utilizó una columna Phenomenex (Phenogel $5 \mu \mathrm{m}$ Linear) de dimensiones 7.8 x $300 \mathrm{~mm}$. El disolvente de elución fue Hexafluoroisopropanol (HFIP) + $10 \mathrm{mM}$ de trifluoroacetato de sodio. El flujo fue de $1 \mathrm{~mL} / \mathrm{min}$ y la temperatura de $25^{\circ} \mathrm{C}$. La preparación de las muestras se realizó mediante la elución a una concentración del $2 \%$ y se hicieron pasar por un microfiltro de 0,22 $\mu \mathrm{m}$ de PFTE para eliminar cualquier residuo sólido. Se prepararon dos réplicas de cada muestra y la longitud de onda elegida fue de $230 \mathrm{~nm}$.

\section{Densidad}

Los ensayos de densidad se han llevado a cabo por inmersión en agua, empleando una balanza analítica Mettler Toledo AX205 DR. De acuerdo a la norma ISO 1183-1, la densidad final es el valor medio tras 5 mediciones. La realización de estos ensayos permitió el cálculo y la comparación de las densidades de las diferentes matrices de PET utilizadas, tanto del material virgen como del reciclado.

\subsection{DESARROLLO DE UNA CÁMARA DE IMPREGNACIÓN}

\subsubsection{Diseño del proceso de impregnación}

En esta sección se describe el diseño y la puesta a punto, a escala de laboratorio, de la cámara de impregnación. Esta cámara permitirá estudiar el grado de impregnación y adhesión de los materiales desarrollados. A partir de los resultados de este estudio se seleccionarán los materiales más válidos para ser introducidos en el proceso de pultrusión y obtener los perfiles finales. 
La cámara de impregnación se acopla a la salida de la línea de extrusión, de manera que, por un lado de la cámara entra un flujo constante y controlado de matriz y por el otro, la entrada de la fibra (Figura 3.10). De esta manera, la fibra y la matriz se encuentran en la zona central de la cámara, para obtener el preimpregnado. Los parámetros de proceso a ajustar son velocidad de tiro de la fibra, la temperatura de la extrusión y de la cámara, el caudal y la velocidad de extrusión.

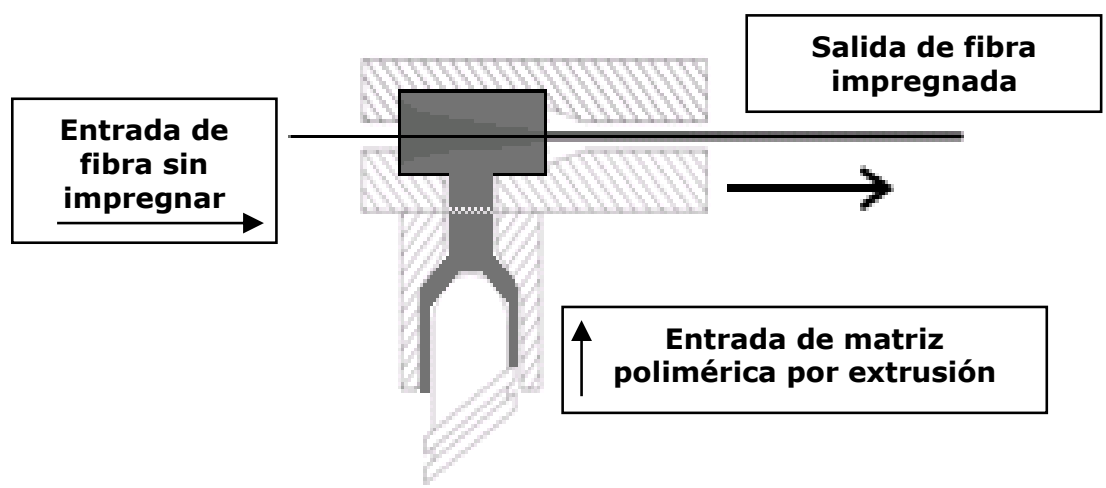

Figura 3.10 Esquema general de la cámara de impregnación situada a la salida de la extrusora

\subsubsection{Desglose de los componentes del proceso de impregnación}

El prototipo de cámara de impregnación que fue diseñada para cumplir nuestro objetivo constó de los siguientes elementos:

- Diseño del dispositivo de guiado para la correcta alimentación de la fibra de vidrio.

- Diseño y/ó adaptación del pre-calentador: Es importante considerar el secado de las fibras de refuerzo que tiene lugar en la zona de precalentamiento, antes de que se encuentren con la matriz. Es necesario, por consiguiente, fijar una zona de precalentamiento en la cámara para mejorar la impregnación del termoplástico en los hilos de la fibra.

- Cámara de impregnación: La configuración de la cámara es también un factor importante en el proceso de impregnación. El propósito de la cámara de impregnación es consolidar la masa de matriz termoplástica y la fibra, a la vez que es impregnada hasta un estado en el que existe una distribución uniforme de fibras dentro de la matriz termoplástica. Para hacer esto, se diseñó una cámara caliente para inducir una acumulación de presión adecuada a medida que la masa de matriz termoplástica/fibra progresa a través de la cámara, siendo el molde suficientemente largo para permitir un tiempo adecuado de contacto entre la matriz y la fibra. En la Figura 3.11 se muestra un esquema de la cámara. 


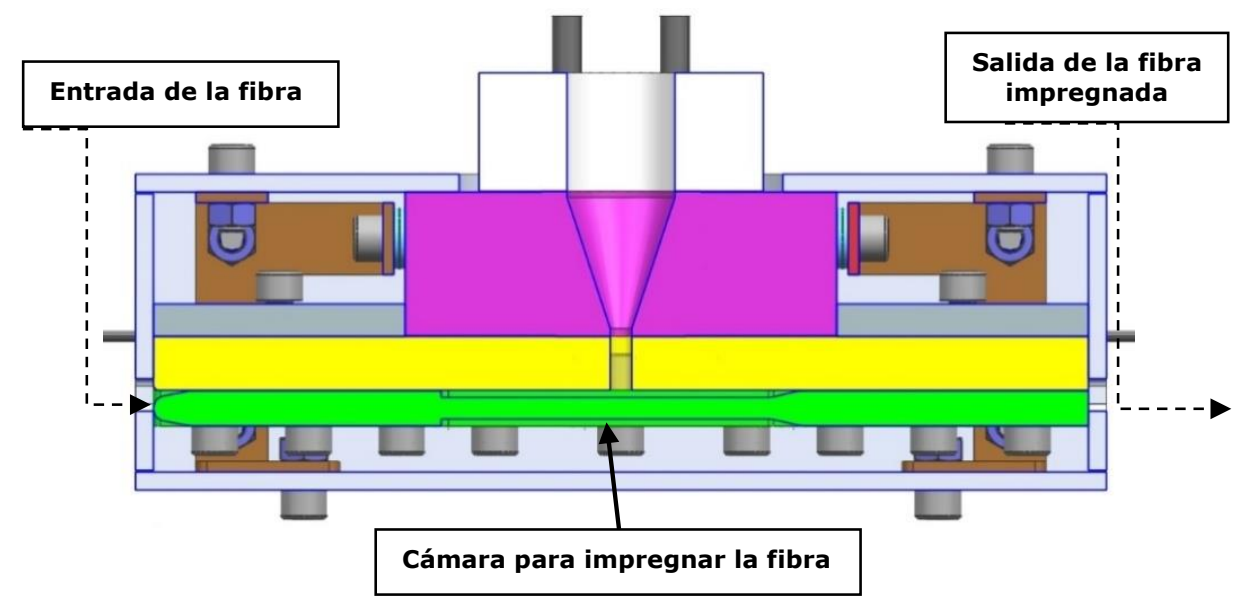

Figura 3.11 Esquema general de la cámara de impregnación

- Por último, se requiere de un dispositivo de refrigeración: Se incorporó un dispositivo de enfriamiento a la salida del cabezal de impregnación para asegurar un producto estable y dimensional. El dispositivo que se instaló fue un cañón de flujo de aire.

Una vez construidos estos elementos, se pasó a la realización del montaje de la cámara de impregnación. La cámara está compuesta de cinco elementos clave mostrados en la Figura 3.12:

En primer lugar, dispone de un acople con cuatro pistones para instalar la cámara a la boquilla de la línea de extrusión. Este acople presenta un orificio central en forma cónica por donde pasaría el material fundido de la extrusora al molde de impregnación. Antes del molde de impregnación se dispone de una placa calefactada por 4 resistencias que se soportan por 4 tapas. Esta placa se ajusta al acople mediante 6 tornillos centrales.

A continuación, se integra el molde de impregnación, con dimensiones de $260 \times 70 \mathrm{~mm}^{2}$, a la placa calefactada mediante 18 tornillos. El molde presenta un estrechamiento en la entrada y en la salida para evitar fugas del material fundido. En la zona central más ancha, se produce el encuentro de la matriz fundida y la fibra y tiene unas dimensiones de $150 \times 10 \mathrm{~mm}^{2}$. La temperatura del molde es medida por medio de dos termopares individuales situados a la entrada y a la salida del molde. Por último, se incorpora una superficie alrededor de la cámara fabricada de mica, que protege del calor desprendido por la cámara, así como de posibles escapes de matriz fundida. 


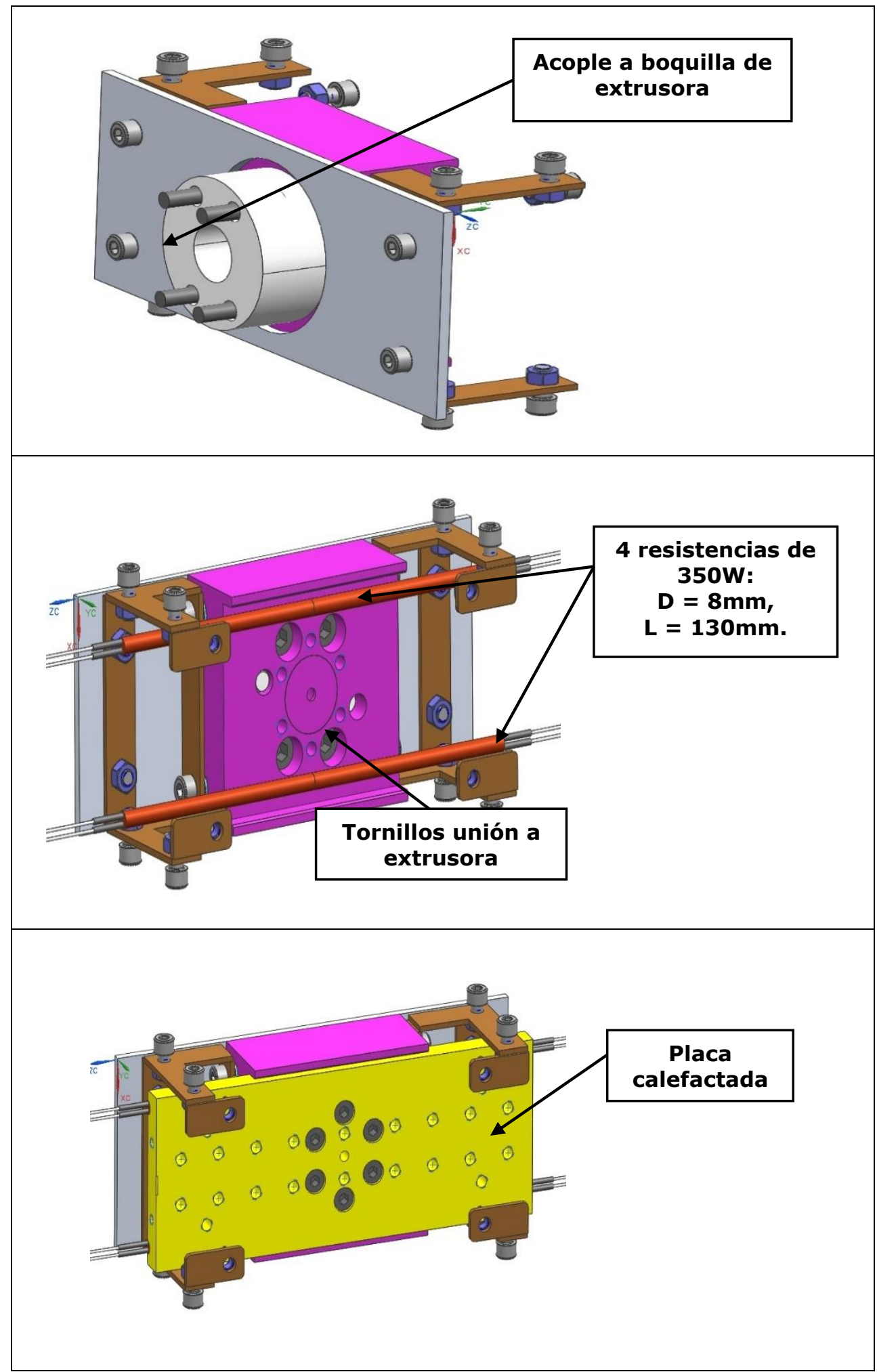




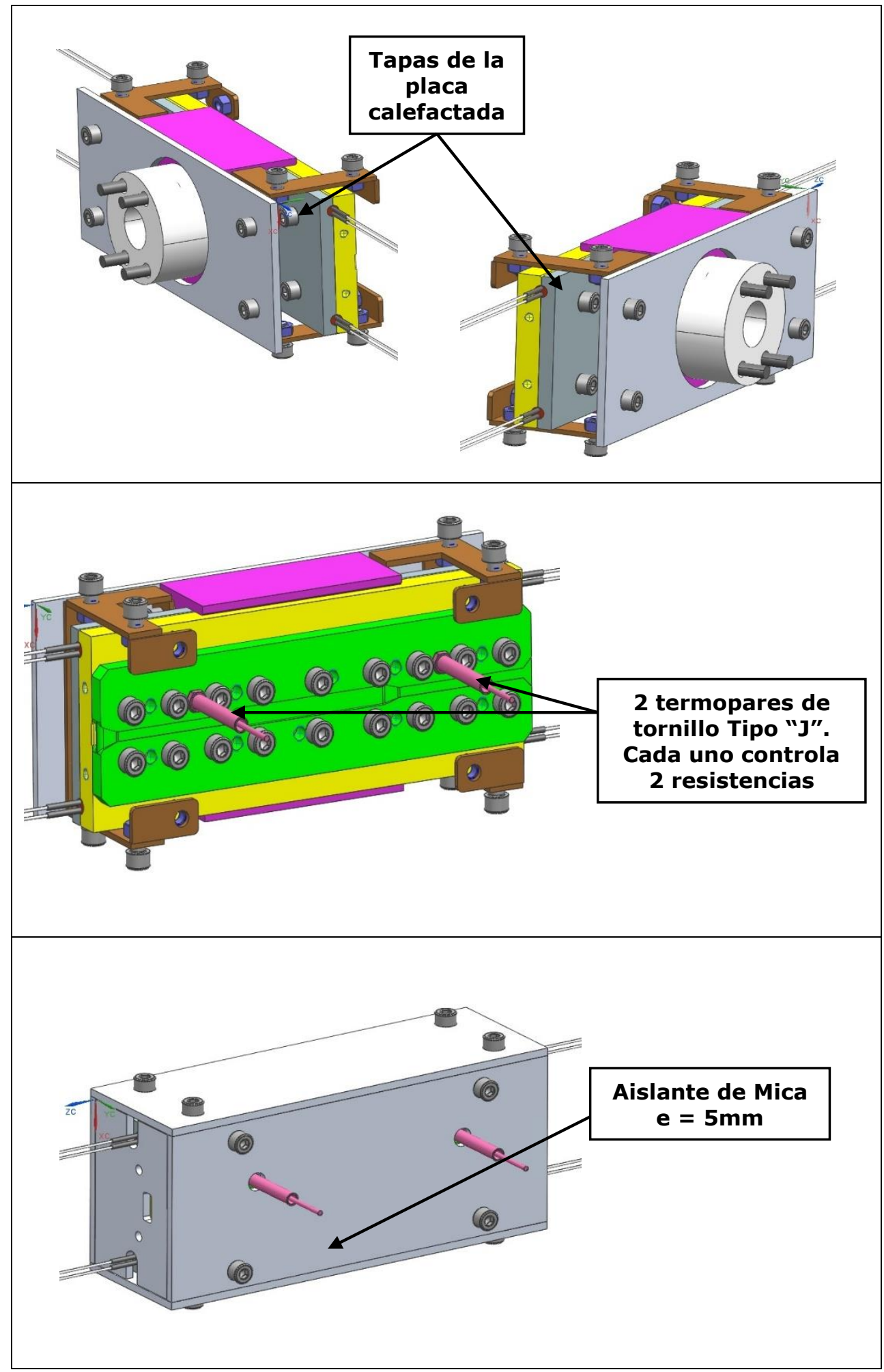

Figura 3.12 Componentes de la cámara de impregnación 


\subsection{LÍNEA DE PULTRUSIÓN EN FUNDIDO}

La obtención de los perfiles a partir de la pultrusión en fundido (con material en forma de granza) fue realizada en la empresa CirComp. La tecnología de pultrusión utilizada se muestra en la Figura 3.13. Esta tecnología permite llevar a cabo la impregnación y la consolidación en una sola etapa. CirComp ha trabajado en los últimos años en la pultrusión y termoformado de elementos estructurales utilizando poliamidas como matrices termoplásticas. En sus trabajos anteriores se investigaron varios tipos de poliamidas, como la PA6, para determinar la viscosidad y la reología necesarias para optimizar la calidad y la velocidad del proceso ${ }^{1}$.

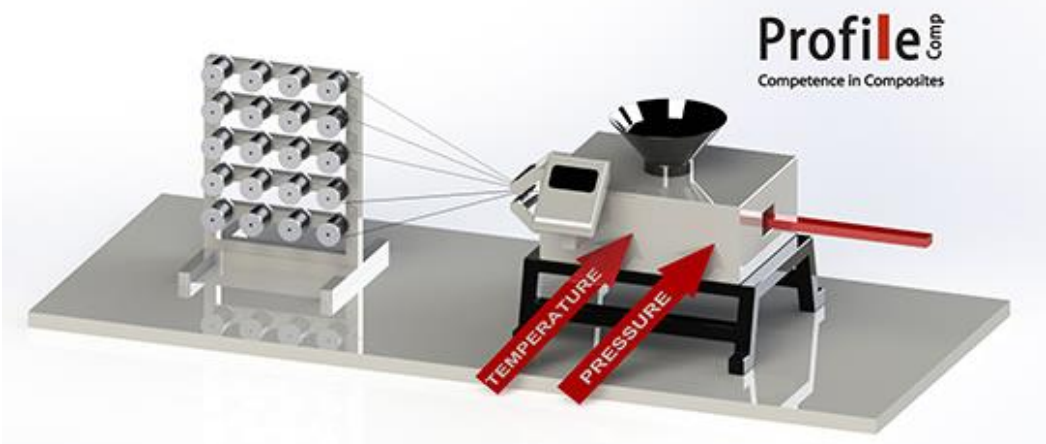

Figura 3.13 Línea de pultrusión en fundido

En esta investigación se evaluaron dos materiales de PET de procedencia residual con distinta viscosidad. De este estudio se seleccionó uno de ellos para la producción de los perfiles termoplásticos pultruidos.

\subsection{LÍNEA DE PULTRUSIÓN EN POLVO}

\subsubsection{Towpregger (equipo de impregnación en polvo)}

La máquina towpregger produce los towpreg, que son pre-impregnados de fibra de vidrio y la matriz en forma de polvo ${ }^{2,3}$. Esta máquina esta patentada y se encuentra en la Universidad de ISEP-School of Engineering de Oporto ${ }^{4}$. Como se explicó en el Capítulo anterior, los trabajos de este grupo de investigación se han centrado hasta ahora en la pultrusión de polipropileno (PP), a partir del material comercial en forma de polvo, sin someter al PP a ninguna modificación reológica. En la Figura 3.14 se muestra las imágenes de la máquina de towpregger y el esquema general de las etapas que se llevan a cabo para la obtención del preimpregnado. 

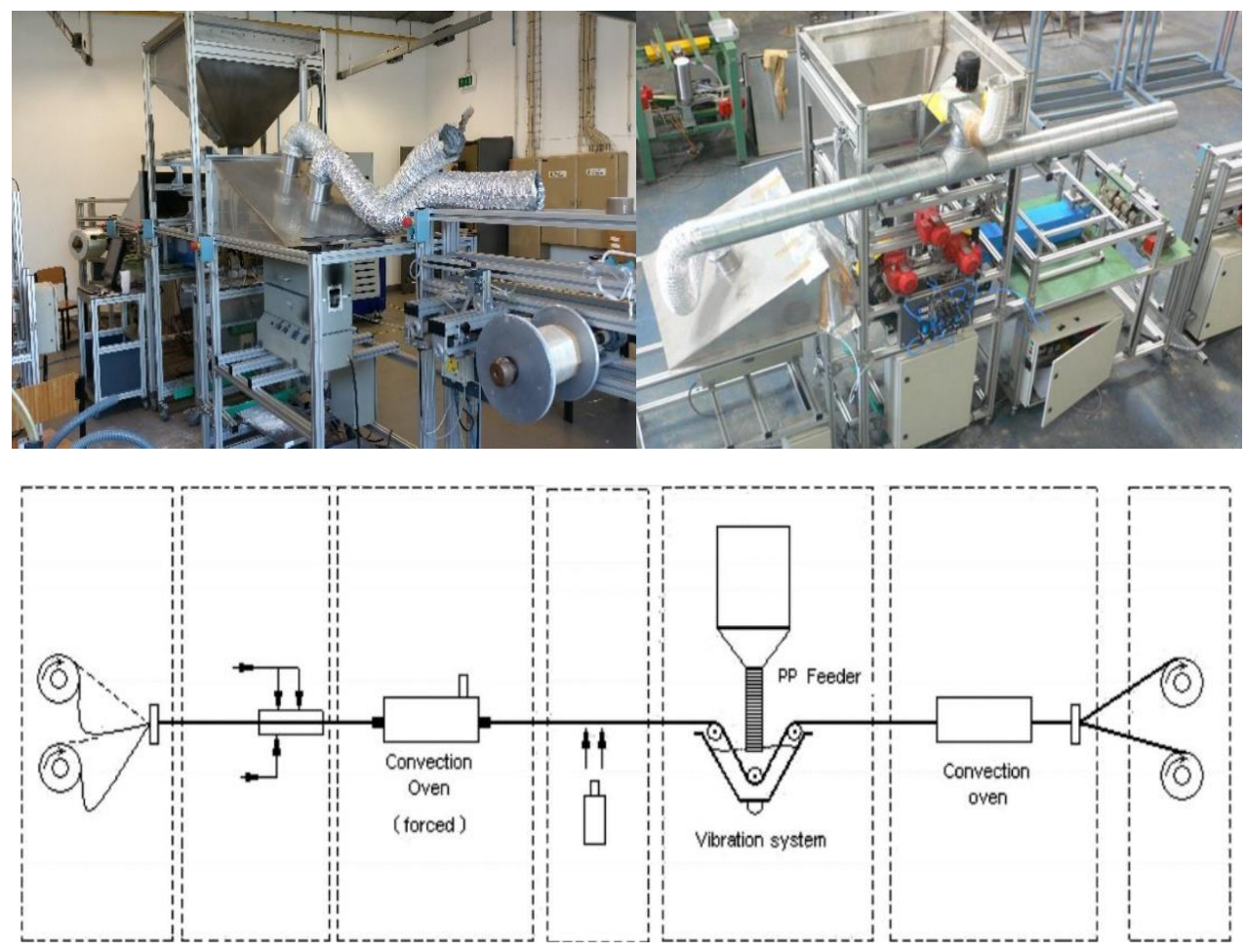

Wind-off Spreader Heating section Coating section Consolidation Wind-up

Figura 3.14 Imágenes y esquema general de la línea de impregnación en polvo ${ }^{5}$

El funcionamiento de esta máquina consta de los siguientes seis pasos principales:

1. En primer lugar, la mecha de fibra de vidrio se desenrolla de una gran bobina ("windd-off"). Para ello es necesario controlar la calidad de las fibras durante este proceso, ya que a veces el hilo del roving se separa de los demás y se enreda en la máquina.

2. Posteriormente, el roving entra en un separador o peine ("spreader") que extiende y separa las fibras con aire comprimido regulado. Las fibras entran como un conjunto de hilos y salen como cientos de hilos de fibras separados. Este proceso sirve para facilitar la etapa de calentamiento que le sigue y el recubrimiento de las fibras, siendo imprescindible para conseguir una adecuada adherencia de la matriz y la fibra. 


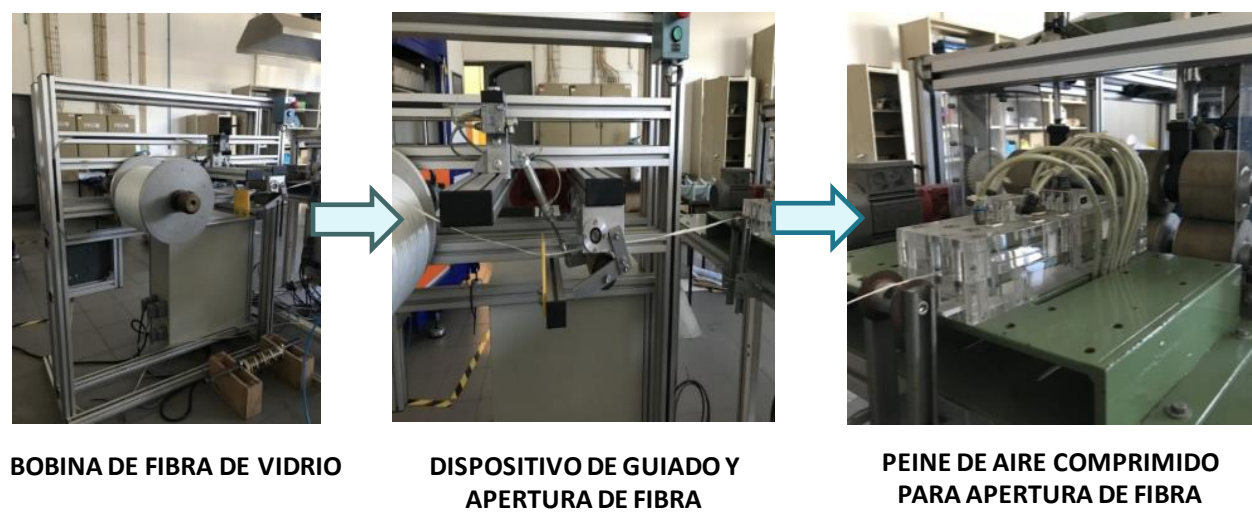

Figura 3.15 Etapa de apertura de la fibra

3. Una vez la hebra está abierta, esta entra en un horno para calentar las fibras ("heating section") (Figura 3.16). Este precalentamiento es necesario para que se produzca la impregnación de la matriz termoplástica en polvo en los hilos de la fibra. Parte importante de los ensayos realizados, estuvieron asociado a encontrar esta temperatura óptima de pre-calentamiento con los materiales estudiados.

4. Las fibras calientes entran en un recipiente lleno con la matriz empleada ("coating section") donde se mezcla el polvo con las fibras. Las fibras deben estar a una temperatura óptima para que el polvo se reblandezca. Posteriormente, la hebra impregnada con el polvo entra en un segundo horno, que funde el polímero que se ha depositado en las fibras ("consolidation").

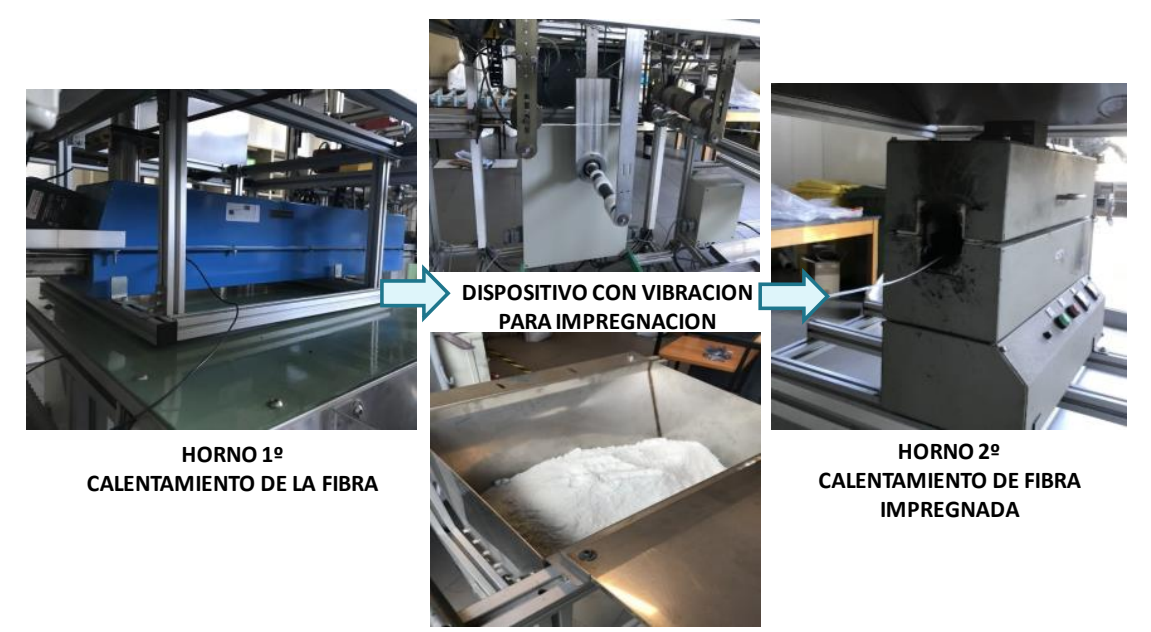

Figura 3.16 Etapa de impregnación de la fibra con el material en polvo 
5. Finalmente, con el polímero fundido en las fibras, obtenemos el towpreg, que se recoge enrollado en una bobina grande ("wind-up") (Figura 3.17). La velocidad de los distintos rotores del proceso y las temperaturas de los hornos (horno 1 y 2 ) es controlada por un ordenador y fueron otras de las variables que se ajustaron para los materiales estudiados.

6. Como último paso, es necesario traspasar el towpreg de la bobina grande en bobinas pequeñas de 30 metros. Estas bobinas se colocan sobre un soporte de guiado para posteriormente ser conducidas al proceso de pultrusión (Figura 3.17).

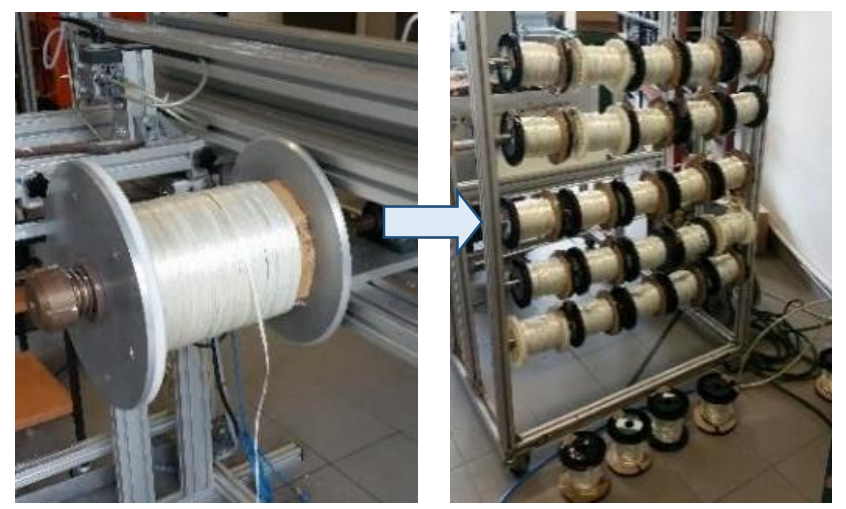

Figura 3.17 Dispositivo de guiado de bobinas

\subsubsection{Máquina de pultrusión}

Una vez que se obtienen los towpreg se lleva a cabo la consolidación mediante el proceso de pultrusión. A partir de la máquina de pultrusión (ver Figura 3.18) se obtiene un perfil pultruido (perfil compuesto). Este proceso se divide en seis pasos:

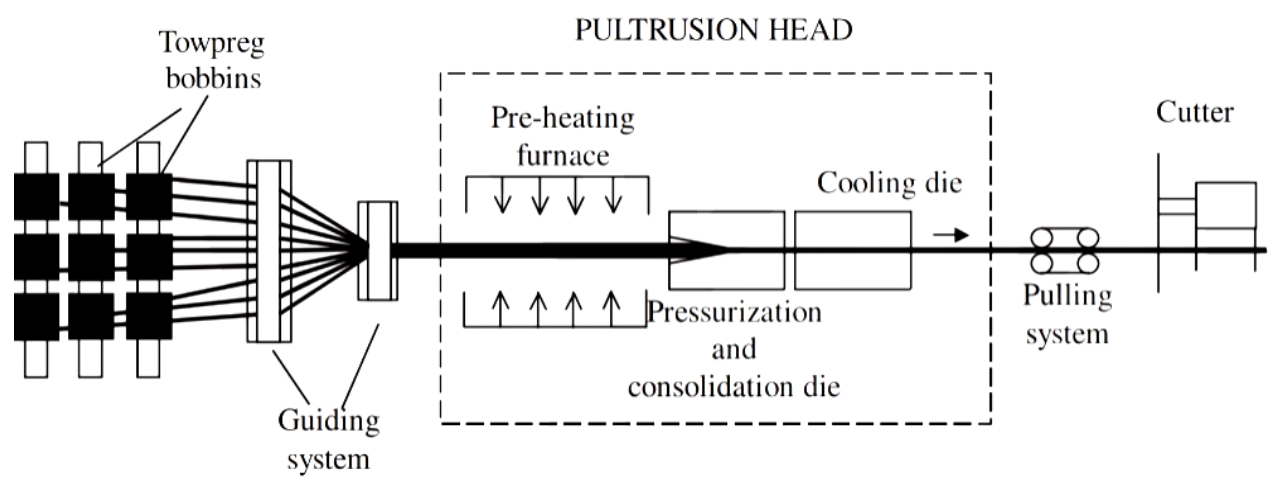

Figura 3.18 Esquema de la línea de pultrusión ${ }^{5}$ 
1. El towpreg de las bobinas soportado en las barras ("towpreg bobbins") se va desenrollando para entrar en el horno de precalentamiento (Figura 3.19). La temperatura óptima de este horno, de acuerdo al material estudiado, también fue optimizado.

2. Un número determinado de towpregs es reagrupado haciéndose pasar por un dispositivo de guiado ("guiding system"). El número de towpregs depende del porcentaje de fibra que se quiera obtener en el perfil compuesto. Posteriormente, son guiados al horno de precalentamiento ("pre-heating furnace") donde se calientan para reblandecer el polímero depositado en las fibras (Figura 3.19).

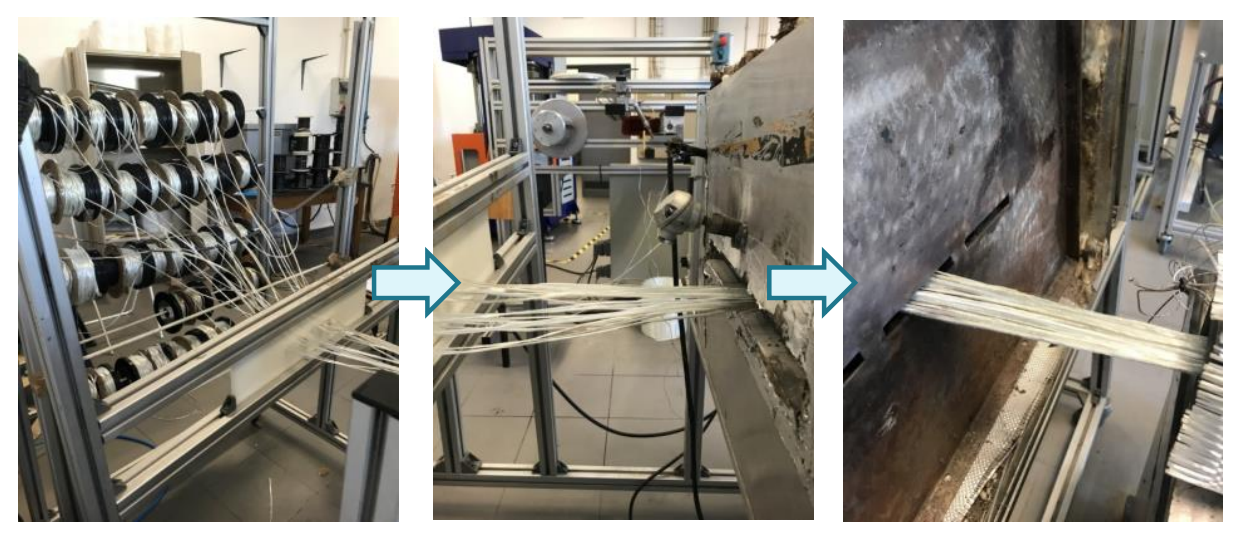

Figura 3.19 Guiado y calentamiento del towpreg

3. Al salir del horno, los towpreg entran en el molde de pultrusión ("pressurization and consolidation die"), calefactado por cuatro resistencias donde se produce la consolidación. Las fibras funden con el polímero, se comprimen y los polímeros sueldan y consolidan las fibras. Para comprimir las fibras, la entrada del molde es más grande que la salida.

4. Próximo al molde de pultrusión caliente se encuentra un molde de refrigeración ("cooling die") enfriado por un sistema de circulación de agua. El perfil sale del molde caliente de pultrusión y entra al molde frío donde se solidifica el perfil. Finalmente, se obtiene el perfil final pultruido (Figura 3.20).

5. Durante todo el proceso es importante mantener los towpreg y el perfil traccionados. El sistema de tracción recíproco o tirador ("pulling system") hace que el perfil pultruido salga del molde de una manera continua y constante (Figura 3.20). 
6. Por último, los perfiles son cortados ("cutter") en función de los tamaños requeridos para su caracterización. Hay un panel de control de pantalla táctil que se utiliza para programar y controlar la temperatura del molde de pultrusión y el refrigerador y la velocidad de tiro del "pulling system". En esta parte del proceso de pultrusión fue donde se concentraron los esfuerzos para ajustar los parámetros, acorde a los materiales estudiados.
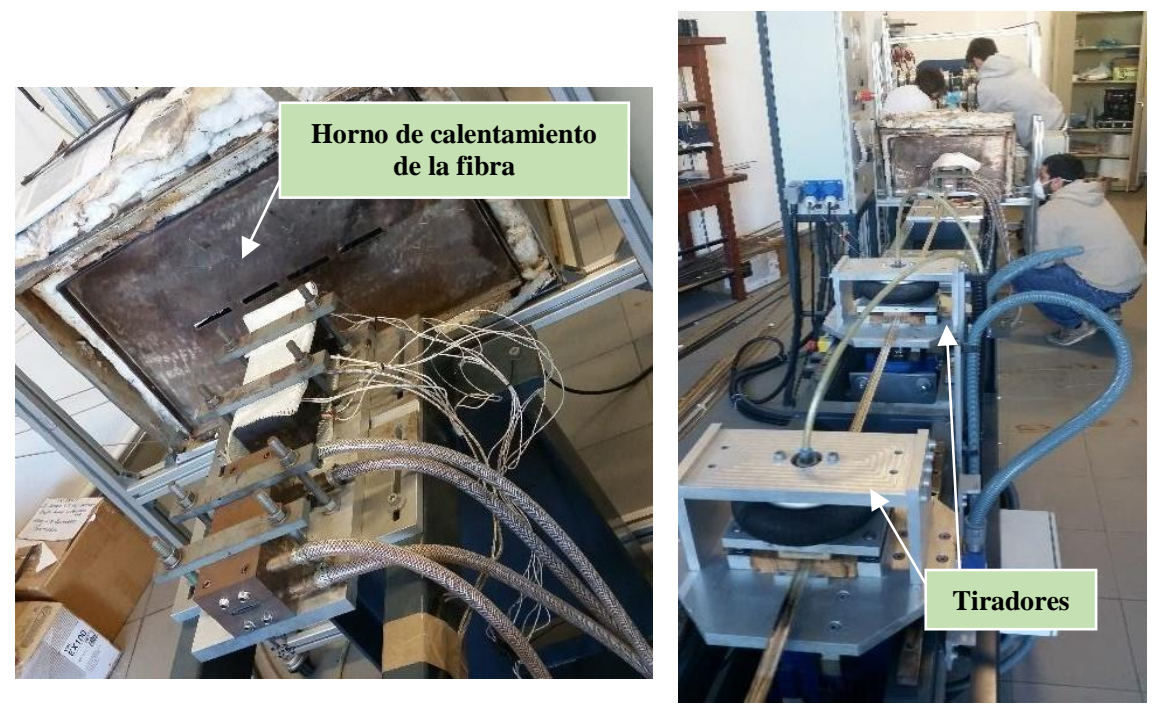

Figura 3.20 Molde calefactado seguido de molde refrigerado y dispositivo tirador

\subsection{TÉCNICAS DE CARACTERIZACIÓN DE PERFILES}

\subsubsection{Comportamiento mecánico}

La caracterización mecánica de los perfiles obtenidos por pultrusión en fundido y en polvo se basó en ensayos de tracción y flexión.

\section{Ensayo de Tracción}

Los ensayos de tracción se llevaron a cabo en un equipo Shimadzu AG-X usando una celda de $100 \mathrm{kN}$. La velocidad de ensayo fue de $2 \mathrm{~mm} / \mathrm{min}$ según la norma ISO 527. La distancia entre las mordazas fue de $200 \mathrm{~mm}$ y el extensómetro fue de $50 \mathrm{~mm}$. Las dimensiones de los perfiles se muestran en la Tabla 3.2. 
Tabla 3.2 Dimensiones de la probeta de ensayo de tracción

\begin{tabular}{l|l|cc}
\hline & & \\
\hline
\end{tabular}

\section{Ensayo de Flexión}

El equipo Shimadzu AG-X dispone de dos soportes distanciados $80 \mathrm{~mm}$. Se aplica una carga en el centro de la probeta a una velocidad determinada y al igual que en el ensayo de tracción se registra la gráfica esfuerzo-deformación. Las propiedades de flexión de los perfiles se determinaron bajo la norma ISO 14125.

Este método se utilizó para investigar el comportamiento de flexión de los perfiles y determinar la resistencia a la flexión y el módulo de flexión de éstos. La distancia entre los soportes fue de $80 \mathrm{~mm}$ y la velocidad de ensayo fue de $2 \mathrm{~mm} / \mathrm{min}$. Las dimensiones de los perfiles se muestran en la siguiente Tabla.

Tabla 3.3 Dimensiones de la probeta de ensayo de flexión

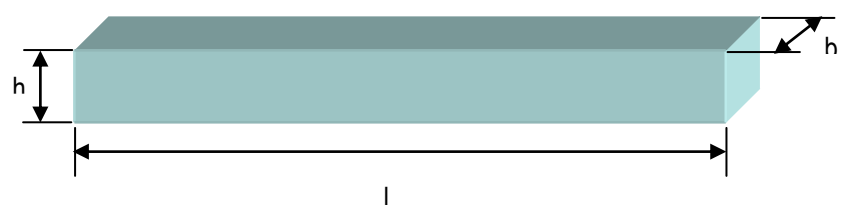

Medidas perfil pultrusión en fundido Medidas perfil pultrusión en polvo $(\mathrm{mm})$

\begin{tabular}{c|l|cc}
\hline I & Longitud & $\mathbf{8 0}$ & $\mathbf{8 0}$ \\
b & Anchura & $\mathbf{1 5}$ & $\mathbf{2 0}$ \\
$\mathrm{h}$ & Espesor & $\mathbf{2}$ & $\mathbf{2}$ \\
\hline
\end{tabular}




\subsubsection{Microscopía}

La microscopía electrónica de barrido (SEM) se usó para determinar la calidad de impregnación y evaluar la distribución de fibra y la adhesión de fibra / matriz de los perfiles obtenidos en el Capítulo 5. Las observaciones se llevaron a cabo en un microscopio FESEM Hitachi H-7000. Previamente a la realización del ensayo, las muestras se recubrieron con una capa delgada de aleación de oro y paladio para hacerlas conductoras.

\subsubsection{Otras técnicas instrumentales de caracterización}

\section{Calcinación}

El porcentaje en volumen de fibra puede determinarse mediante la calcinación de los perfiles, a partir de la siguiente expresión.

$$
\omega f=\frac{m_{3}-m_{1}}{m_{2}-m_{1}}
$$

Donde $m_{1}, m_{2}$ y $m_{3}$ son:

$m_{1}$ masa inicial del crisol seco.

$m_{2}$ masa inicial del crisol seco más el espécimen seco.

$m_{3}$ masa final de crisol más residuo tras la calcinación.

El contenido de masa de fibra de vidrio en los pre-impregnados y los perfiles obtenidos se determinó mediante pruebas de calcinación de acuerdo con la norma EN ISO 1172. La calcinación de la muestra se llevó a cabo dentro de un crisol en un horno a $625^{\circ} \mathrm{C}$ durante 15 minutos. Además, al conocer las densidades de fibra y polímero, $\rho_{f}$ y $\rho_{f}$, respectivamente, la fracción de masa de fibra $\left(\omega_{f}\right)$ se puede convertir en fracción de volumen de fibra $\left(v_{\mathrm{f}}\right)$ mediante la expresión:

$$
v_{f}=\frac{\omega_{f} / \rho_{f}}{\omega_{f} / \rho_{f}+\left(1-\omega_{f}\right) / \rho_{p}}
$$




\subsection{REFERENCIAS BIBLIOGRÁFICAS}

1. Gardiner G. More details on MAI Skelett design process. https://www.compositesworld.com/blog/post/more-details-on-mai-skelett-designprocess.

2. Silva FJG, Amorim E, Baptista A, Pinto G, Campilho RDSG, Castro MRA. Producing hybrid pultruded structural products based on preforms. Compos Part B Eng. 2017;116:325-332. doi:10.1016/j.compositesb.2016.10.070

3. João Silva, João Nunes, C. A. Bernardo AM. Thermoplastic Matrix Composites from Towpregs.; 2013. doi:http://dx.doi.org/10.5772/46845

4. Machado Gomez Da Silva JF. Equipment to produce continuosly powder coated thermoplastic matrix prepregs (towpregs). 2002.

5. Novo PJ, Silva JF, Nunes JP, Marques AT. Pultrusion of fibre reinforced thermoplastic pre-impregnated materials. Compos Part B Eng. 2016;89:328-339. doi:10.1016/j.compositesb.2015.12.026

6. De Moura Giraldi ALF, De Jesus RC, Mei LHI. The influence of extrusion variables on the interfacial adhesion and mechanical properties of recycled PET composites. J Mater Process Technol. 2005;162-163(SPEC. ISS.):90-95. doi:10.1016/j.jmatprotec.2005.02.046

7. Badía JD, Vilaplana F, Karlsson S, Ribes-Greus A. Thermal analysis as a quality tool for assessing the influence of thermo-mechanical degradation on recycled poly(ethylene terephthalate). Polym Test. 2009;28(2):169-175. doi:10.1016/j.polymertesting.2008.11.010

8. Van Kets K, Delva L, Ragaert K. Structural stabilizing effect of SEBSgMAH on a PPPET blend for multiple mechanical recycling. Polym Degrad Stab. 2019;166:60-72. doi:10.1016/j.polymdegradstab.2019.05.012

9. Spinace MAS. Characterization of Poly ( ethylene terephtalate ) after. 2001:20-25.

10. Elamri A, Lallam A, Harzallah O, Bencheikh L. Mechanical characterization of melt spun fibers from recycled and virgin PET blends. J Mater Sci. 2007;42(19):8271-8278. doi: 10.1007/s10853-007-1590-1

11. Elamri a, Abid K, Harzallah O, Lallam a. Characterization of Recycled/ Virgin PET Polymers and their Composites. Am J Nano Res Appl Am J Nano Res Appl Spec Issue Nanocomposites Coat Manuf. 2015;3(11):11-16. doi:10.11648/j.nano.s.2015030401.13

12. Farah S, Kunduru KR, Basu A, Domb AJ. Molecular Weight Determination of Polyethylene Terephthalate. Elsevier Inc.; 2015. doi:10.1016/B978-0-323-313063.00008-7

13. Sanches NB, Dias ML, Pacheco EBAV. Comparative techniques for molecular weight evaluation of poly (ethylene terephthalate) (PET). Polym Test. 2005;24(6):688-693. doi:10.1016/j.polymertesting.2005.05.006 


\section{Ca pítu I o 4}

Como se explicó anteriormente, para poder utilizar materiales termoplásticos como materia prima en procesos de pultrusión, es necesario que cumplan con unas propiedades determinadas, especialmente, que posean baja viscosidad. Así pues, el presente capítulo tiene como objetivo investigar técnicas de modificación reológica que permitan modificar la viscosidad del PET de una manera controlada, sin que éstas induzcan pérdidas en las propiedades mecánicas. En primer lugar, se presentan los materiales utilizados (polímeros y aditivo) así como su caracterización completa. Posteriormente se muestra el procedimiento experimental de cada una de las modificaciones y la respectiva caracterización reológica, térmica y mecánica de las muestras obtenidas.

\section{Materiales, modificación reológica y caracterización}

\subsection{MATERIALES}

En esta sección se describen los materiales poliméricos utilizados en la investigación: el PET virgen y dos corrientes de PET reciclado y su caracterización completa en términos morfológicos, reológicos, térmicos y mecánicos. Finalmente, se muestra el aditivo empleado en el método de modificación química.

\subsubsection{Matrices}

En la Tabla 4.1 se muestran las matrices de PET donde figura el nombre comercial, la procedencia y el formato físico en el que fueron suministradas.

Tabla 4.1 Descripción de los materiales de PET utilizados

\begin{tabular}{ccc}
\hline Muestra & Descripción & Formato \\
\hline $\begin{array}{c}\text { PET virgen } \\
\text { (VPET) }\end{array}$ & $\begin{array}{c}\text { PET virgen comercial } \\
\text { (NovaPET Spirit L21) }\end{array}$ & Suministrado en granza \\
$\begin{array}{c}\text { PET reciclado } \\
\text { (rPET blue) }\end{array}$ & $\begin{array}{c}\text { PET post-consumo triturado y lavado } \\
\text { procedente del reciclador }\end{array}$ & Suministrado en escamas \\
$\begin{array}{c}\text { PET reciclado } \\
\text { (rPET color) }\end{array}$ & $\begin{array}{c}\text { PET post-consumo triturado y lavado } \\
\text { procedente del reciclador }\end{array}$ & Suministrado en escamas \\
\hline
\end{tabular}


EI PET virgen fue seleccionado por sus propiedades específicas de baja viscosidad y bajo peso molecular. Fue suministrado por NOVAPET, empresa española productora de resinas de PET. Este grado de PET virgen ha sido utilizado para el estudio de la degradación termo-mecánica e hidrolítica, así como para la realización de las mezclas PET reciclado y PET virgen (modificación física). Este material es denominado como VPET (material de PET virgen que no ha pasado por ningún proceso posterior de extrusión).

Las corrientes de PET reciclado que fueron utilizadas proceden de residuos de PET post-consumo (rPET) y se dividen en dos categorías: rPET blue y rPET color. En las siguientes imágenes se puede observar el aspecto físico de las escamas de las dos corrientes de rPET. Para su caracterización, estas escamas de PET reciclado fueron extruidas a $265^{\circ} \mathrm{C}, 100 \mathrm{rpm}$ y $5 \mathrm{Kg} / \mathrm{h}$.
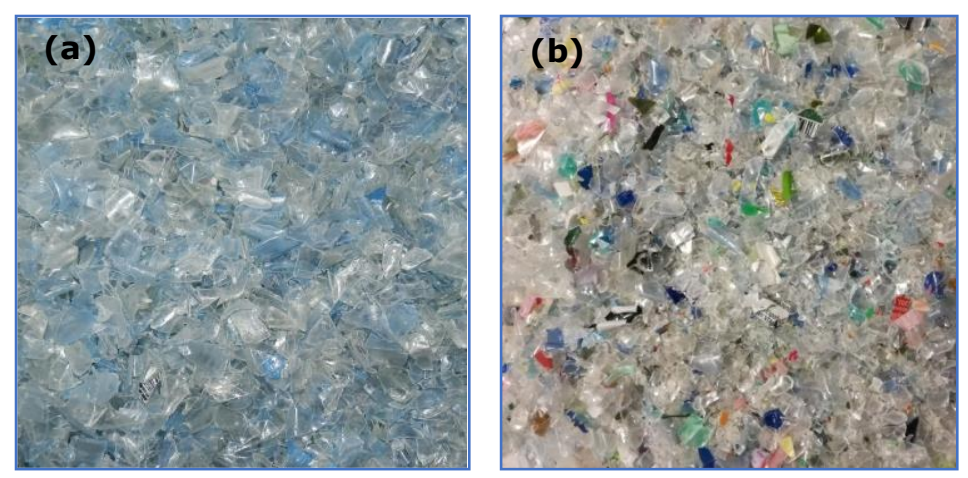

Figura 4.1 Imagen de escamas de rPET blue (a) y rPET color (b)

El primero (rPET blue), proviene de una fuente más limpia que contiene en su mayoría residuos de botellas azules y transparentes. Las escamas de rPET blue fueron utilizadas para la realización de las mezclas de PET reciclado/PET virgen (modificación física).

El rPET color proviene de una corriente más contaminada, que puede estar formada por escamas de PET coloreadas y multicapa, procedente de varios tipos de envasado. El rPET color no fue sometido a procesos de modificación para disminuir su viscosidad, ya que, como se verá posteriormente en su caracterización, este material poseía, por sí solo, valores bajos de viscosidad. Por este motivo el rPET color fue utilizado directamente tras ser extruido como materia prima para la fabricación de perfiles (mostrados en el siguiente Capítulo). 
Esta propiedad de baja viscosidad sugiere una ventaja en la utilización de estos residuos como matrices para la fabricación de perfiles por pultrusión, ya que, como se ha explicado con anterioridad, una viscosidad baja supone una mejor impregnación. Sin embargo, este residuo de PET que proviene de residuos coloreados y multicapa presenta desventajas, como son unas pobres propiedades mecánicas (baja tenacidad) en comparación con otros residuos de PET coloreados más limpios (rPET blue).

Es importante tener en cuenta que las pobres propiedades mecánicas que puedan presentar los materiales reciclados pueden solventarse, ya que, en los perfiles pultruidos, la matriz juega un papel menor (aunque importante, porque es quien transmite el esfuerzo a las fibras) frente al conjunto de fibras, que son quienes aportan la mayoría de propiedades mecánicas. Esto hace posible que se puedan utilizar estos materiales residuales, especialmente el rPET color, para la fabricación de perfiles pultrusión.

\subsubsection{Caracterización de los materiales de PET}

En esta sección se describen las características morfológicas, térmicas y mecánicas de los polímeros de rPET extruidos y del VPET. De esta manera, podemos conocer las propiedades de partida que presentan las matrices en función de si son vírgenes o reciclados y en función de la vertiente de residuos que provengan.

\section{Análisis espectroscópico FTIR}

Como se explicó anteriormente, en muchos casos los materiales reciclados presentan pequeñas proporciones de contaminación de residuos de otra base polimérica. Por este motivo, con el objetivo de confirmar la homogeneidad y la procedencia polimérica de sus componentes se realizó un estudio espectroscópico de los materiales de rPET (rPET blue y rPET color). Para llevar a cabo el análisis se realizó un muestreo mediante una extracción selectiva por color, es decir, se analizan los diferentes colores de escamas existentes en las muestras.

En el análisis del material reciclado rPET blue se encontraron seis tipos de escamas diferentes colores (diferentes tonalidades de azules opacos y translúcidos y escamas transparentes). En la Figura 4.2 se compara el espectro de absorbancia de las escamas que presentaba el rPET blue y la muestra de PET virgen (VPET). Como se puede ver en la Figura, todas las escamas mostraron las mismas bandas que la muestra de $\mathrm{VPET}^{1,2}$. De esta manera, se verificó que las 
escamas presentes en el reciclado rPET blue eran de base polimérica poliéster (PET).

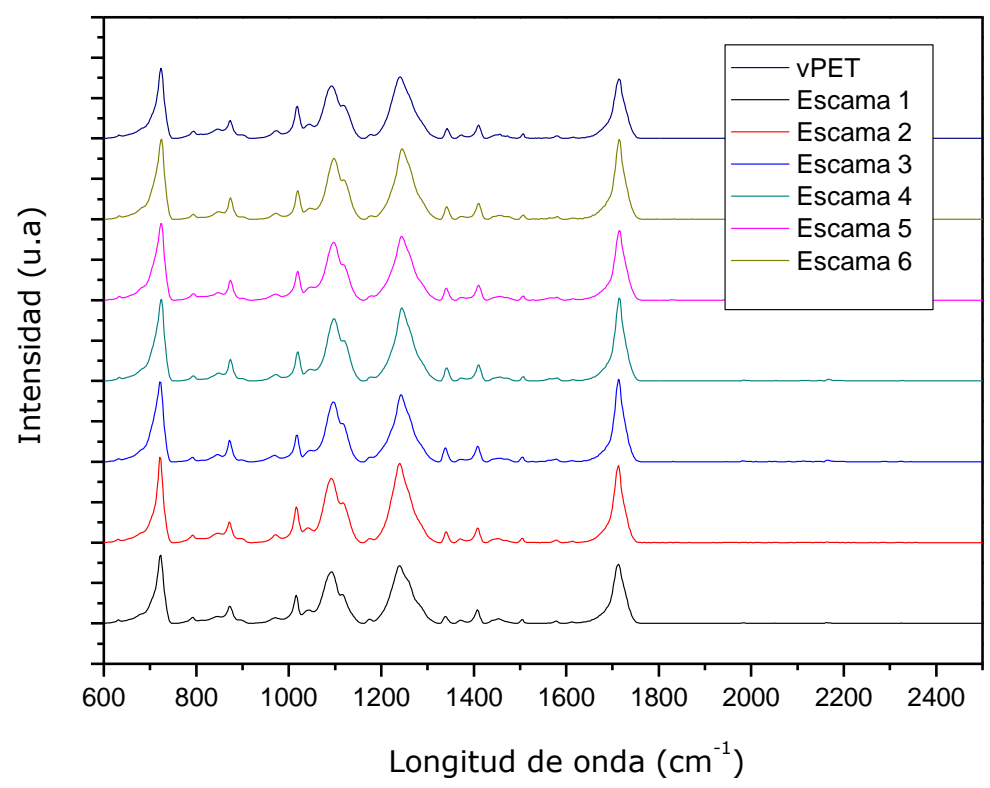

Figura 4.2 FTIR de PET virgen y escamas de rPET blue

A continuación, se analizó la muestra de reciclado $\mathrm{rPET}$ color donde se encontraron fragmentos de distintas tonalidades (ver Figura 4.3). La mayoría de las escamas que constituían la muestra eran trasparentes y azul claro, con la presencia, en muy poca proporción, de otras tonalidades de azules, verdes, blancas, etiquetas, y diversos colores.

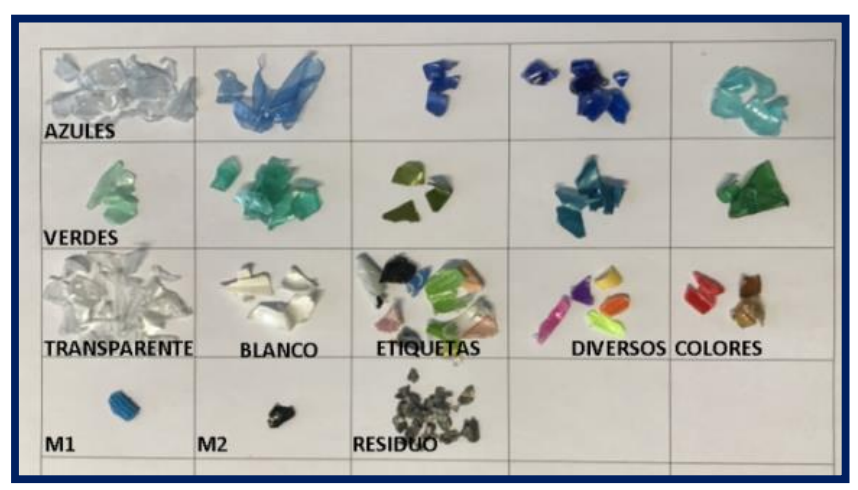

Figura 4.3 Extracción selectiva de rPET color 
En la Figura 4.4 se muestra el espectro de las diferentes escamas encontradas en rPET color. Como resultado del análisis espectroscópico se determinó que todas las escamas correspondían al espectro del PET excepto las denominadas M1 y M2. En el caso de M1 (espectro rosa de la Figura 4.4) corresponde a un polietileno (PE), que podría proceder a tapón de botella. En el caso de M2 (espectro verde) se trata de un Poliestireno (PS), utilizado en bandejas de carne, fruta, etc ${ }^{3}$.

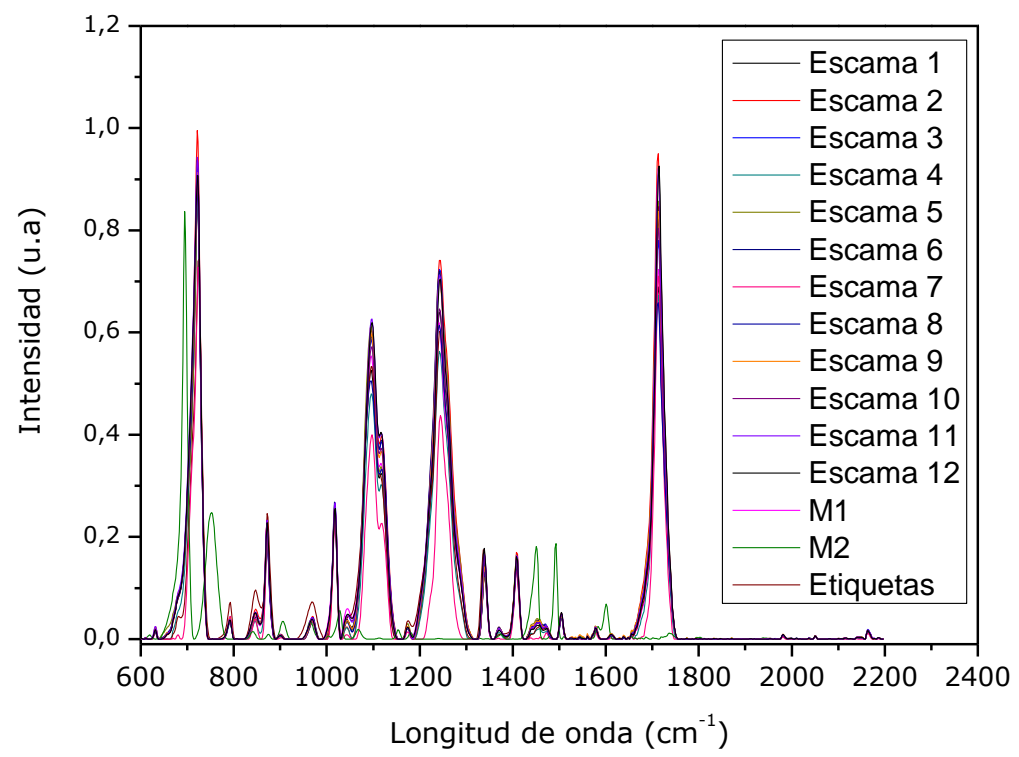

Figura 4.4 FTIR de escamas de rPET color

\section{Propiedades térmicas}

Los valores de las propiedades térmicas de las muestras de PET, como la temperatura de transición vítrea $\left(T_{g}\right)$, la temperatura de fusión $\left(T_{f}\right)$ y la temperatura de cristalinidad $\left(T_{c}\right)$, así como la cristalinidad $(X)$ se muestran en la Tabla 4.2.

Tabla 4.2 Propiedades térmicas de los materiales PET de partida

\begin{tabular}{ccccc}
\hline Muestra & $\mathbf{T}_{\mathbf{g}} \pm \mathbf{0 . 5}\left({ }^{\circ} \mathbf{C}\right)$ & $\mathbf{T}_{\mathbf{f}} \pm \mathbf{0 . 5}\left({ }^{\circ} \mathbf{C}\right)$ & $\mathbf{T}_{\mathbf{c}} \pm \mathbf{0 . 5 ( { } ^ { \circ } \mathbf { C } )}$ & $\boldsymbol{X} \pm \mathbf{1}(\%)$ \\
\hline vPET & 80.1 & 244.6 & 166.7 & 27 \\
rPET blue & 81.1 & 245.3 & 186.5 & 27 \\
rPET color & 82.8 & 251.4 & 196.4 & 26 \\
\hline
\end{tabular}


Los resultados sugieren que, independientemente de la procedencia, la $T_{g}$ es similar en todas las muestras de PET ( $\pm 3 \%)$. En el caso de la $\mathrm{T}_{f}$ los valores del VPET y rPET blue son similares; esto puede ser debido a que rPET blue es una de las corrientes más limpias y similar a un material vPET virgen. El rPET color presentó la $T_{f}$ más alta, pudiendo ser debido a la presencia de contaminantes de otros polímeros, como se comprobó anteriormente en el análisis FTIR.

Las diferencias más significativas entre los materiales de PET, las vemos en la $T_{c}$, la cual es más alta en los materiales reciclados como en el rPET blue $\left(20^{\circ} \mathrm{C}\right.$ mayor que vPET) y en el rPET color $\left(30^{\circ} \mathrm{C}\right.$ mayor). El aumento de la $\mathrm{T}_{\mathrm{c}}$ en los materiales reciclados de debe a la degradación a la que son sometidos durante su reprocesado, lo que provoca que la cristalización comience a una temperatura más alta ${ }^{4}$. En cuanto al grado de cristalinidad no se apreciaron diferencias significativas.

\section{Propiedades reológicas}

A continuación, se llevó a cabo el análisis reológico de las muestras de PET. En primer lugar, se estudió la viscosidad mediante reometría dinámica. En la Figura 4.5 se presenta la viscosidad compleja de las muestras frente a la temperatura en la que se puede confirmar cómo el PET virgen (VPET), seleccionado por sus características de alta fluidez, presenta menores valores de viscosidad que el material reciclado rPET blue.

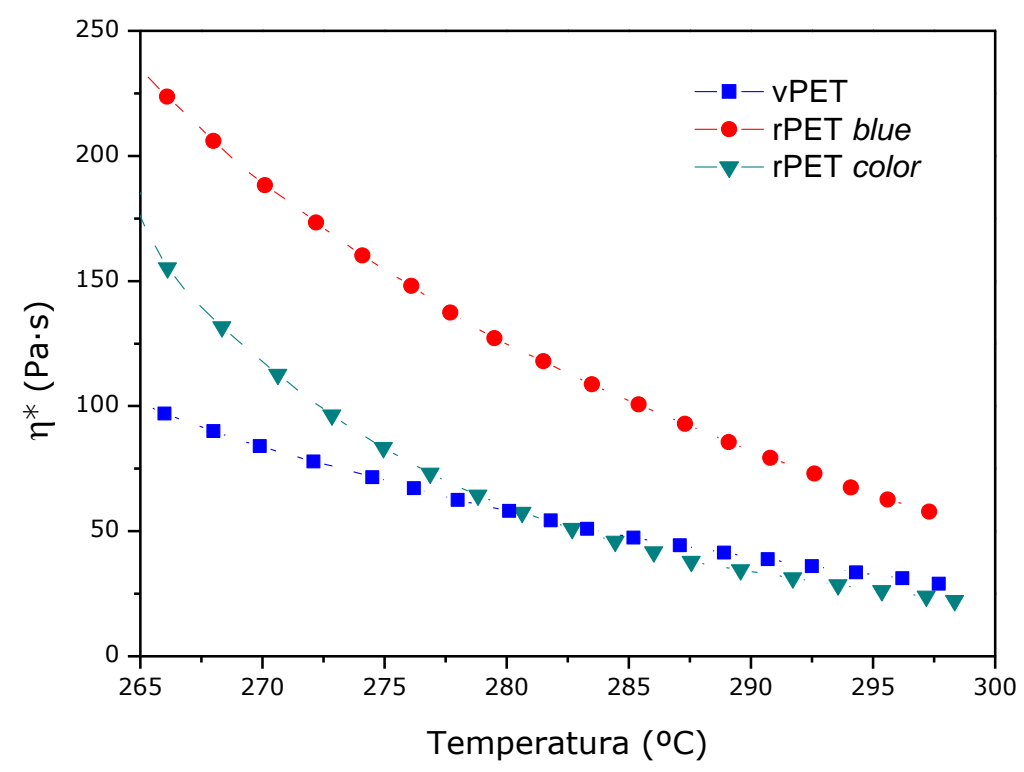

Figura 4.5 Viscosidad compleja de los materiales de PET 
Si comparamos los materiales de PET de origen reciclado (rPET blue y rPET color), podemos ver como el material reciclado rPET color es mucho más fluido que el rPET blue. Esto puede deberse a que el rPET color contiene contaminación de materiales multicapa y coloreados, los cuales provocan una mayor degradación y en consecuencia una menor viscosidad.

Como se comentó anteriormente, el peso molecular ( $\mathrm{Mw}$ ) de los polímeros está intrínsecamente relacionado con la viscosidad de éstos ${ }^{5}$. En la Figura 4.6 se muestran los valores del Mw de los materiales de PET que fueron calculados por GPC. Estos resultados mostraron cómo el rPET blue (material más viscoso) presenta mayor peso molecular. A pesar de que el VPET y el rPET color presentaron valores similares en viscosidad, el rPET color presentó menor peso molecular, lo cual nos indica que tiene cadenas poliméricas más cortas.

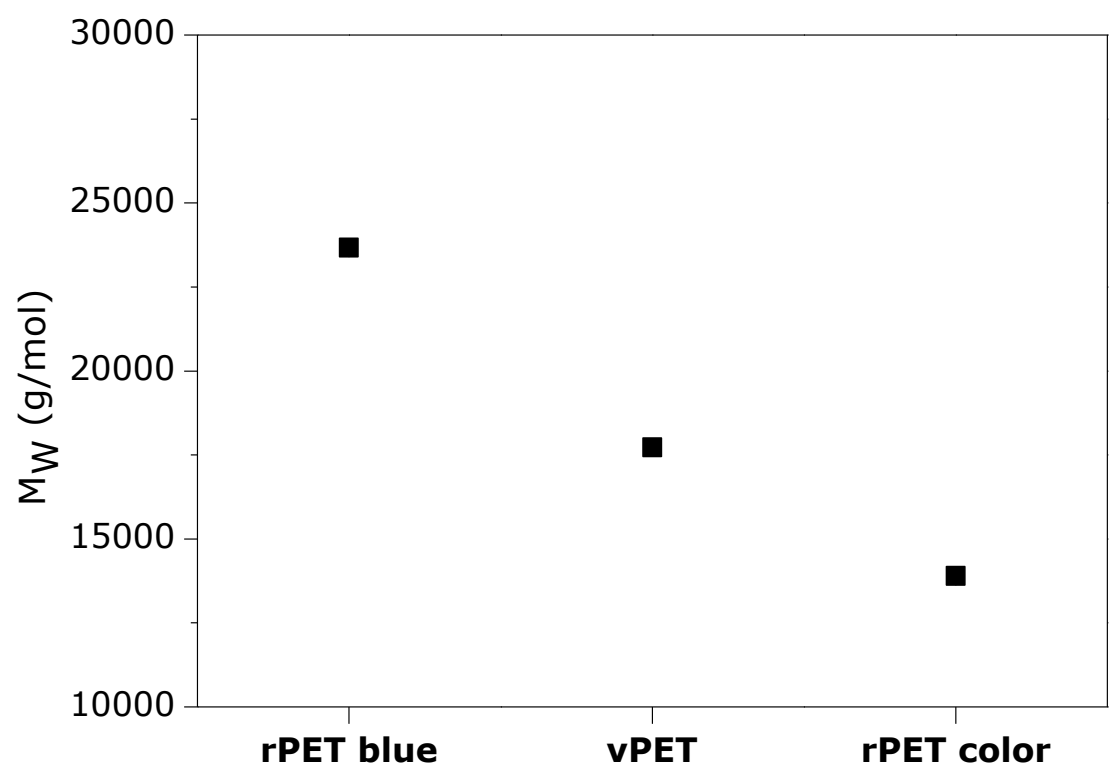

Figura 4.6 Peso molecular de los materiales de PET 


\section{Propiedades mecánicas}

Por último, se evaluaron las propiedades mecánicas de los materiales de PET a partir del Módulo de Young y el Impacto Charpy (Tabla 4.3).

Tabla 4.3 Propiedades mecánicas de los materiales utilizados

\begin{tabular}{ccc}
\hline Muestra & $\begin{array}{c}\text { Módulo de Young } \\
(\mathbf{M P a})\end{array}$ & $\begin{array}{c}\text { Impacto Charpy } \\
\left(\mathbf{K J} / \mathbf{m}^{\mathbf{2}}\right)\end{array}$ \\
\hline VPET & $2396 \pm 36$ & $9.07 \pm 1.48$ \\
rPET blue & $2301 \pm 10$ & $2.02 \pm 0.38$ \\
rPET color & $2579 \pm 84$ & $0.58 \pm 0.03$ \\
\hline
\end{tabular}

A partir de estos resultados se pudo comprobar que el valor del Módulo de Young no disminuye en los materiales reciclados, llegando a ser, incluso, en el caso del rPET color, un material más rígido que uno virgen.

Las diferencias más significativas se apreciaron en los resultados del Impacto Charpy, donde los materiales reciclados resultaron ser menos tenaces que el material virgen. Este comportamiento es conocido, ya que, en los materiales poliméricos, siempre que aumenta el Módulo (más rígidos) disminuye el Impacto.

\subsubsection{Aditivo}

El aditivo utilizado en la modificación química fue el ácido 5 amino isoftálico $\left(\mathrm{C}_{8} \mathrm{H}_{7} \mathrm{NO}_{4}\right)$ adquirido en formato de polvo a Sigma-Aldrich. En la Figura 4.7 se muestra la fórmula molecular, la cual contiene grupos terminales amino y ácidos carboxílicos. El aditivo fue secado a $60^{\circ} \mathrm{C}$ en la deshumidificadora antes de su utilización en el método de modificación químico.<smiles>Nc1cc(C(=O)O)cc(C(=O)O)c1</smiles>

Figura 4.7 Fórmula molecular del aditivo utilizado para la modificación química 


\subsection{MODIFICACIÓN REOLÓGICA DE LA MATRIZ POLIMÉRICA}

En esta sección se muestran los diferentes tratamientos de modificación reológica que se llevaron a cabo sobre el PET (expuestos en la Figura 4.8 siguiendo su orden de estudio).

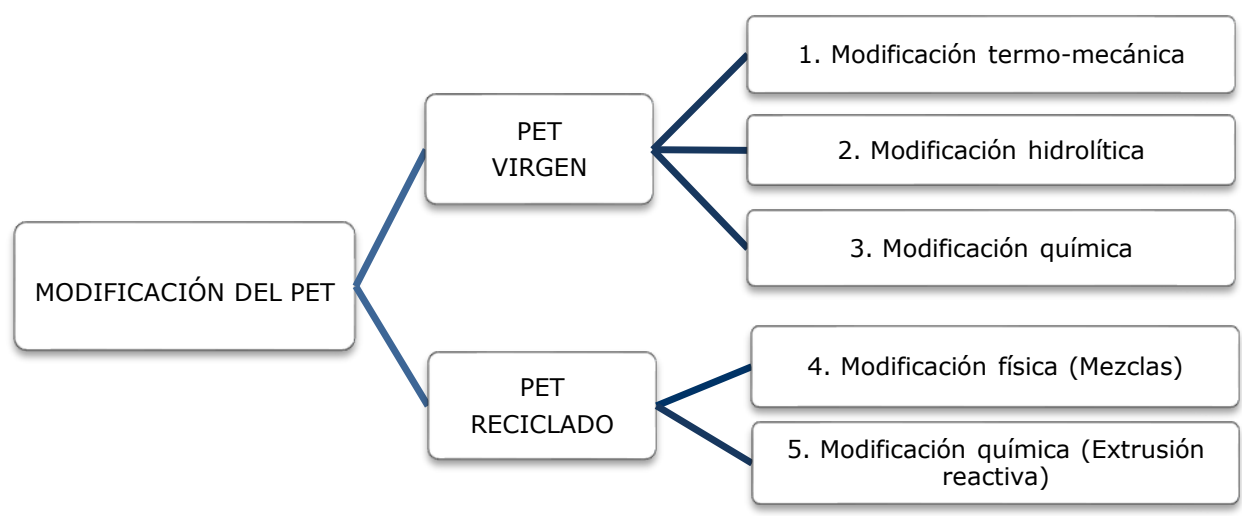

Figura 4.8 Esquema de tratamientos realizados

Como punto de partida, se estudiaron los métodos de modificación reológica basados en tres fenómenos: termo-mecánicos, hidrolíticos y químicos sobre el PET virgen (VPET). El estudio de estos métodos en un material virgen nos permitirá analizar los cambios reológicos y mecánicos que provocan, sin que interfiera el efecto de los contaminantes presentes en el PET reciclado (rPET). De esta primera parte (estudio del efecto de las modificaciones sobre el VPET), se extraen parámetros y conclusiones que posteriormente fueron utilizadas para el tratamiento reológico del rPET.

Posteriormente se llevó a cabo la modificación reológica del rPET (rPET blue) por el método físico y químico. La modificación física consistió en la realización de mezclas de rPET con un vPET de alta fluidez. La modificación química se realizó mediante extrusión reactiva, con el injerto in situ, del aditivo ácido 5 amino isoftálico. El efecto de la modificación química se estudió sobre los compuestos puros y sobre las mezclas (rPET:vPET). Así pues, en este capítulo se muestra el procedimiento experimental de cada una de estas modificaciones y la respectiva caracterización reológica, térmica y mecánica de las muestras obtenidas. 
Todas las modificaciones se llevaron a cabo por extrusión en una extrusora de doble husillo corrotante marca Leistritz modelo $27 \mathrm{GL}$. Previamente a la realización de los experimentos, el PET virgen y el PET reciclado se secaron en la deshumidificadora a $120^{\circ} \mathrm{C}$ durante 3-4 horas.

Durante la extrusión, el PET sufre reacciones de degradación que provocan la escisión de las cadenas poliméricas. Por lo que, para poder entender los cambios macromoleculares y físicos en los que está envuelto el PET, debido a los mecanismos propuestos, es necesario conocer estas reacciones. En la Figura 4.9 se muestran las reacciones principales que experimenta el PET durante la extrusión y las reacciones propuestas que se producen con la incorporación del aditivo en la modificación química.

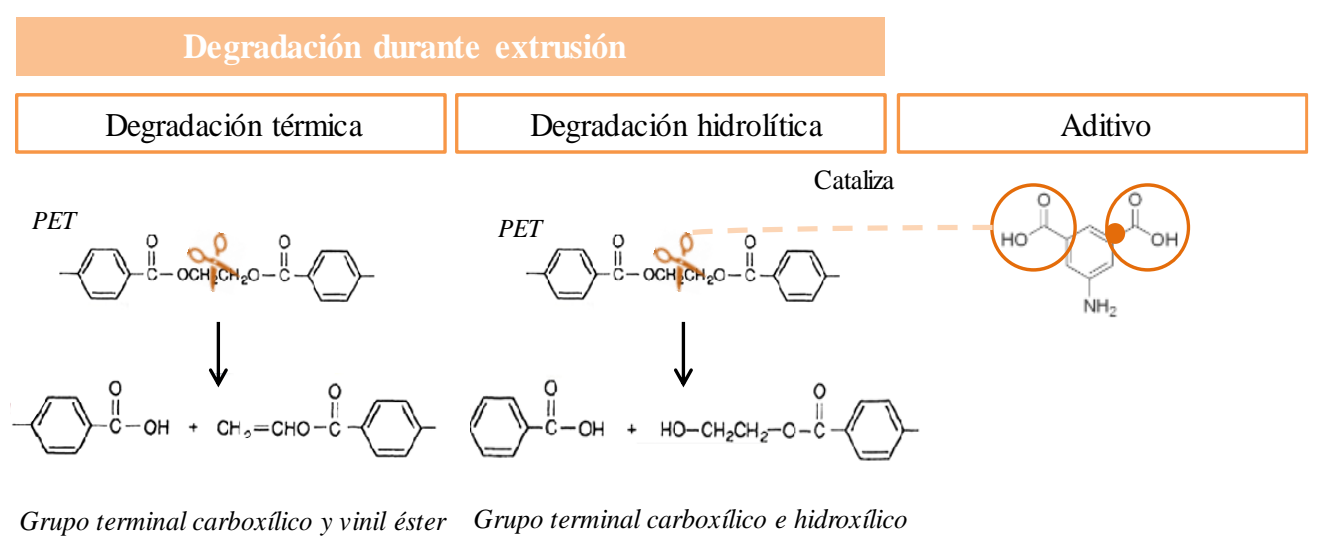

Figura 4.9 Reacciones de degradación térmica, hidrolítica y efecto del aditivo

Las reacciones principales que experimenta el PET durante el proceso de extrusión son reacciones de degradación térmica e hidrolítica ${ }^{6}$. La degradación térmica provoca la escisión de la cadena polimérica, dando lugar a una mayor cantidad de grupos carboxílicos y vinil éster terminales. En el caso de la degradación hidrolítica, la reacción provoca escisiones y la aparición de cadenas con mayor cantidad de grupos terminales carboxílicos y alcoholes ${ }^{7,8}$.

Por otro lado, la incorporación del aditivo (extrusión reactiva) puede actuar como catalizador de estas reacciones, incrementando el efecto que tiene la temperatura y el agua, ya que este aditivo posee grupos carboxílicos capaces de reaccionar7. Además de actuar como catalizadores, los grupos carboxílicos presentes en el aditivo pueden actuar como núcleo ramificador. Estos grupos pueden reaccionar con los grupos carboxílicos (generados por las reacciones de degradación térmica e hidrolítica) para dar lugar a la formación de grupos anhídridos ${ }^{9,10}$. 


\subsubsection{Degradación termo-mecánica del PET virgen}

Como se ha explicado anteriormente, el PET sufre cambios físicos como, por ejemplo, la disminución de la viscosidad cuando es reciclado mecánicamente, debido a la degradación termo-mecánica que se produce en la extrusora. Por este motivo, es muy importante entender y controlar el efecto que tiene el proceso de extrusión sobre la viscosidad de nuestro material, el VPET. El estudio que se muestra en esta sección se basó en la variación de los parámetros de extrusión más relevantes para entender el efecto que tienen sobre la viscosidad y conseguir así, modificar de una manera controlada la viscosidad durante este proceso.

Con el objetivo de obtener la máxima eficiencia, con un número limitado de experimentos, se llevó a cabo un diseño de experimentos (DOE) basado en matrices ortogonales. En esta etapa, se seleccionaron de entre todas las variables de extrusión, aquellas que se consideran significativas según estudios anteriores ${ }^{11}$. A cada una de estas variables significativas se le asigna un rango de variación durante la experimentación (denominados niveles). Como medida del factor de respuesta (output) se seleccionó la viscosidad intrínseca $[\mu]$. Posteriormente, se implementó un estudio estadístico basado en el método del Dr. Taguchi, y de esta forma, poder determinar la influencia de cada una de las variables de extrusión sobre el output elegido, la $[\mu]$. El tratamiento de los datos experimentales se realizó con el software estadístico Minitab17 $7^{12,13}$.

EI DOE fue realizado sobre el VPET con el fin de obtener los datos más representativos posibles. EI PET reciclado fue descartado en este estudio ya que éste puede provenir de diferentes corrientes de residuos y dar resultados muy heterogéneos. Para la elaboración del DOE, en primer lugar, se seleccionaron los siguientes factores: la velocidad del husillo, la temperatura y el caudal ${ }^{5,7,14}$. En la Tabla 4.4 se muestran los factores y niveles seleccionados para la realización del DOE, con tres factores (velocidad de husillo, temperatura y caudal) a dos niveles (con el nivel más alto y el más bajo posible).

Tabla 4.4 Factores y niveles del proceso de extrusión

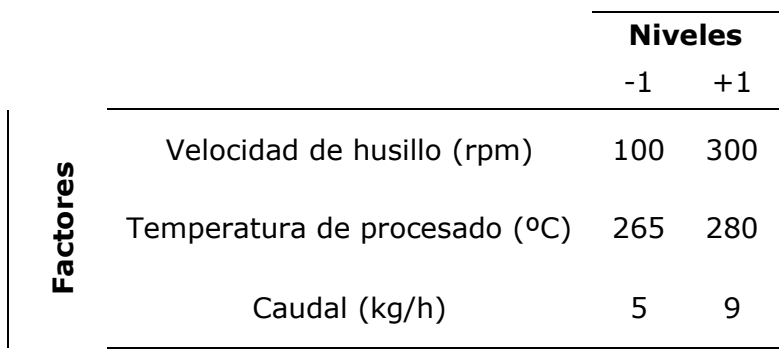


Como resultado del DOE se obtuvieron ocho condiciones experimentales a realizar, que se muestran en la Tabla 4.5. Los experimentos son nombrados en relación al siguiente orden: velocidad de husillo, temperatura y caudal. Estos experimentos llevados a cabo por extrusión son denominados como VPET (1-8).

Tabla 4.5 Matriz de experimentos para la optimización de las condiciones de extrusión

\begin{tabular}{cccc}
\hline Experimento & $\begin{array}{c}\text { Velocidad de husillo } \\
(\mathbf{r p m})\end{array}$ & $\begin{array}{c}\text { Temperatura } \\
\left({ }^{\circ} \mathbf{C}\right)\end{array}$ & $\begin{array}{c}\text { Caudal } \\
(\mathbf{k g} / \mathbf{h})\end{array}$ \\
\hline $\mathbf{1 : 1 0 0 - 2 6 5 - 5}$ & 100 & 265 & 5 \\
$\mathbf{2}: 100-265-9$ & 100 & 265 & 9 \\
$\mathbf{3 : 3 0 0 - 2 6 5 - 5}$ & 300 & 265 & 5 \\
$\mathbf{4}: 300-265-9$ & 300 & 265 & 9 \\
$\mathbf{5 : 1 0 0 - 2 8 0 - 9}$ & 100 & 280 & 9 \\
$\mathbf{6 : 1 0 0 - 2 8 0 - 5}$ & 100 & 280 & 5 \\
$\mathbf{7 : 3 0 0 - 2 8 0 - 9}$ & 300 & 280 & 9 \\
$\mathbf{8 : 3 0 0 - 2 8 0 - 5}$ & 300 & 280 & 5 \\
\hline
\end{tabular}

\subsubsection{Resultados de la degradación termo-mecánica del vPET}

Una vez que se realizó la experimentación y se obtuvo el material de cada experimento (VPET 1-8), se calculó la viscosidad intrínseca $[\mu]$ de cada uno de ellos. Tras introducir los datos resultantes en el software de análisis se obtuvieron los siguientes resultados:

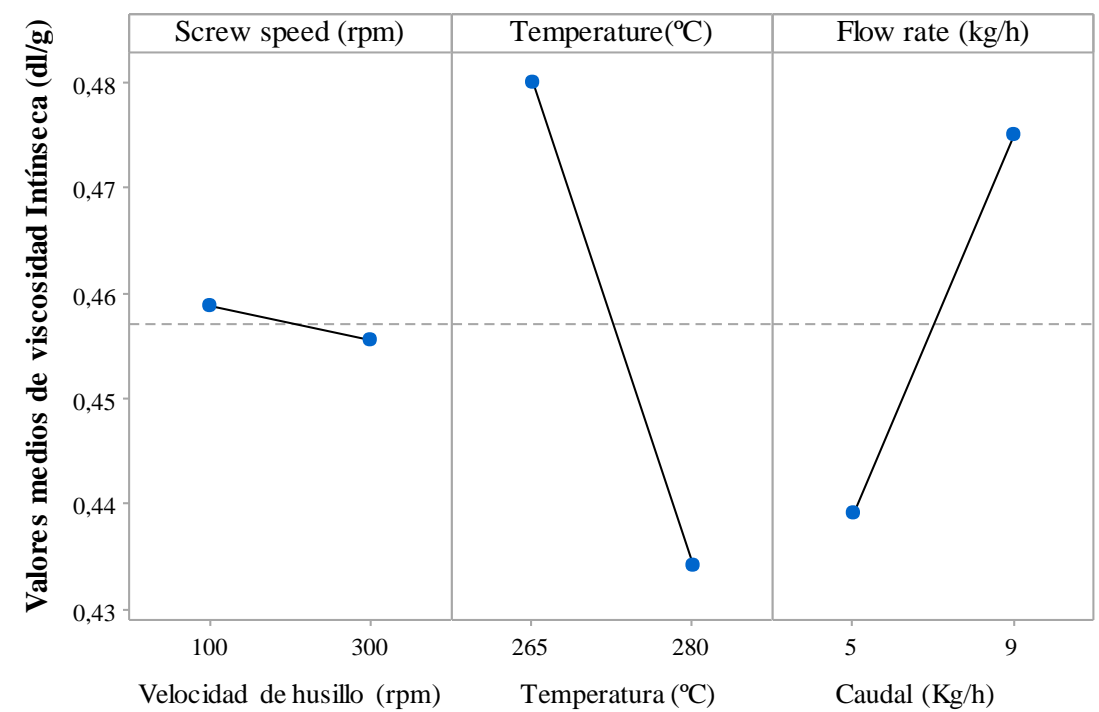

Figura 4.10 Gráfico de efectos principales para los factores de extrusión estudiados 
En la Figura 4.10 se muestra el gráfico de efectos principales del DOE elaborado para el proceso de extrusión del VPET. Cada una de las gráficas cuantifica la influencia del factor sobre la viscosidad mediante la pendiente de la recta que une los dos niveles propuestos. Cuanta más pendiente se obtenga en el gráfico más influye ese parámetro en la viscosidad del VPET. Como se puede apreciar en el gráfico de efectos principales, los parámetros con mayor pendiente son la temperatura y el caudal, lo cual indica que estos factores tienen una fuerte influencia sobre la $[\mu]$ del vPET. También se puede ver cómo la velocidad de husillo, con apenas pendiente, es un parámetro que apenas influye en la $[\mu]$.

Se observa que la muestra extruida a mayor temperatura $\left(280^{\circ} \mathrm{C}\right)$ tiene valores de $[\mu]$ más bajos para cualquiera de los factores de extrusión. Este resultado puede explicarse por las reacciones de degradación térmica que sufre el VPET durante el proceso de extrusión, provocando la escisión de la cadena polimérica (haciendo que las cadenas más cortas fluyan con más facilidad) 5,15. Además de este fenómeno de degradación térmica, la fluidez de los polímeros también depende de la facilidad con la que puedan moverse las cadenas poliméricas y del enmarañamiento entre ellas. Por ello, un aumento de la temperatura permite a las moléculas de cadenas largas desenredarse y fundirse más fácilmente y consecuentemente fluir con más facilidad (reducción de la viscosidad).

En cuanto a la velocidad de alimentación (caudal), la extrusión a un caudal más bajo $(5 \mathrm{~kg} / \mathrm{h})$ mostró los valores más bajos de $[\mu]$. Esto indica que, a un caudal bajo, el tiempo de permanencia del VPET en la extrusora es más largo y, en consecuencia, sufre una degradación térmica más prolongada en el tiempo. De este resultado se puede concluir que el caudal y el tiempo de residencia del VPET, tienen un efecto en la viscosidad similar a trabajar con temperaturas altas.

Estos resultados fueron de gran importancia, ya que a partir de ellos se seleccionaron las condiciones de procesado más adecuadas para ser utilizadas posteriormente en las restantes modificaciones estudiadas. En primer lugar, se seleccionó la velocidad de husillo de 100rpm debido a razones operativas del proceso ya que, a altas velocidades de husillo (300rpm), el hilo de polímero fundido era demasiado fino en la boquilla de la extrusora, lo que no permite un granceado posterior constante. Por otro lado, como se explicó anteriormente, la velocidad de alimentación más baja $(5 \mathrm{~kg} / \mathrm{h})$ permitió obtener valores bajos de viscosidad sin tener que someter al VPET a altas temperaturas. Por este motivo, se seleccionó una temperatura más eficiente $\left(265^{\circ} \mathrm{C}\right)$ junto a un caudal bajo $(5 \mathrm{~kg} / \mathrm{h})$, a partir del cual se pueden obtener materiales con valores bajos de viscosidad. 
Una vez fijadas las condiciones (100rpm, $265^{\circ} \mathrm{C}$ y $\left.\mathbf{5 k g} / \mathbf{h}\right)$, se llevó a cabo la extrusión de los materiales ( $V P E T$ y rPET) para establecer los bancos de experimentación, es decir, de que $[\mu]$ se parte solo por el hecho de extruir los materiales en estas condiciones. En la Figura 4.11 se muestra un diagrama para situar los valores de viscosidad y peso molecular de los polímeros obtenidos en la extrusión. En el centro de la imagen se muestran las aplicaciones principales del PET y qué rango de viscosidad intrínseca se requiere para el moldeo del PET en estas aplicaciones. A la izquierda de la imagen se muestra el rango de peso molecular ( $\mathrm{Mw}$ ) asociado y a la derecha de la imagen, se muestran los resultados de viscosidad intrínseca $[\mu]$ del PET virgen y del PET reciclado extruidos a las condiciones seleccionadas.

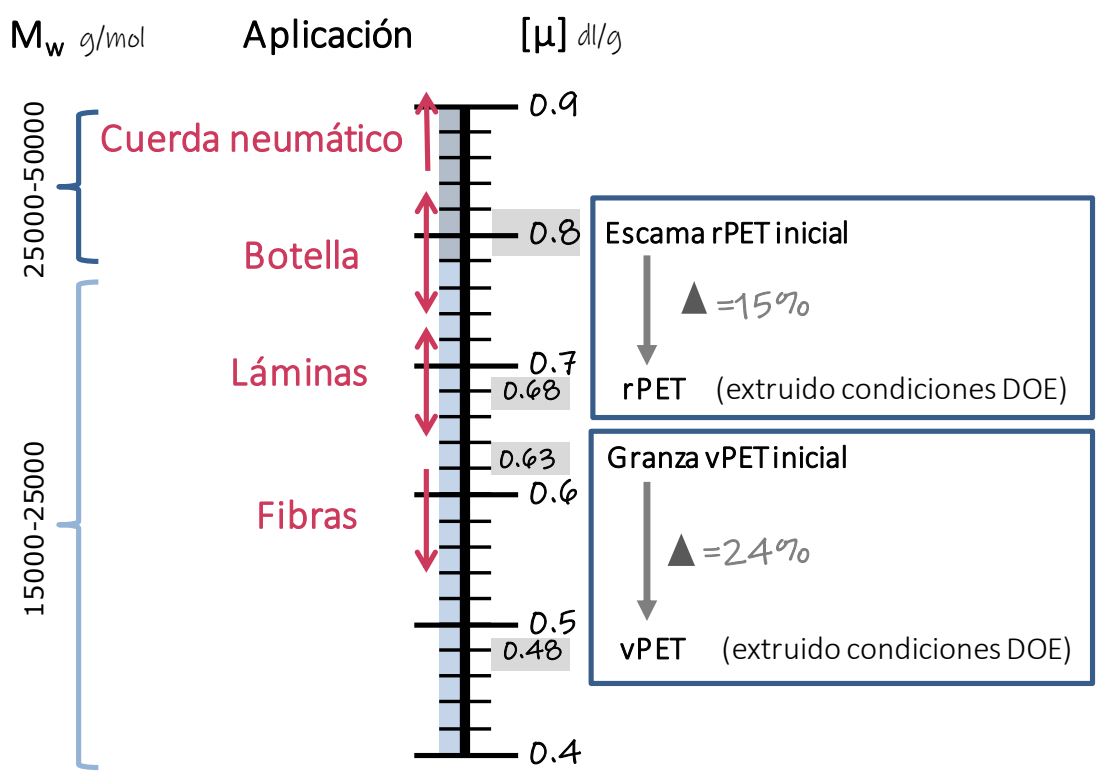

Figura 4.11 Diagrama de viscosidad y rangos de peso molecular de los materiales de partida y modificados

Como era esperado, el resultado de la extrusión produjo la disminución de los valores de $[\mu]$ en ambos materiales. El vPET disminuyó desde $0.63 \mathrm{dl} / \mathrm{g}$ hasta $0.48 \mathrm{dl} / \mathrm{g}(24 \%)$ y el $\mathrm{rPET}$ de $0.80 \mathrm{dl} / \mathrm{g}$ a $0.68 \mathrm{dl} / \mathrm{g}(15 \%)$. El vPET presentó mayor sensibilidad a la degradación termo-mecánica, esto puede deberse a que este material virgen presenta menor peso molecular que el rPET (Figura 4.6), consiguientemente, sus cadenas poliméricas más cortas son más susceptibles a esta degradación termo-mecánica. 


\section{Caracterización de muestras DOE}

Una vez establecidos estos blancos "óptimos", se presentan a continuación los resultados de la caracterización de cada una de las muestras obtenidas en la ejecución del DOE, con el objetivo de estudiar los cambios físicos que experimenta el VPET al ser sometido a la degradación termo-mecánica. Para ello se caracterizaron las muestras VPET 1-8, en términos de viscosidad compleja $\left(n^{*}\right)$, viscosidad intrínseca $[\mu]$, peso molecular viscosimétrico $(\mathrm{Mw})$, índice de fluidez (MFI), propiedades térmicas y mecánicas. Estos resultados se comparan con las propiedades del PET sin extruir (VPET).

\section{Análisis reológico}

En primer lugar, se analizó la influencia de la degradación termo-mecánica en la viscosidad en fundido mediante el ensayo de reometría dinámica de VPET (0$8)$. En la Figura 4.12 se muestran los valores de la viscosidad compleja ( $\left.\eta^{*}\right)$ en función de la temperatura y de la frecuencia $(\omega)$ de estas muestras.
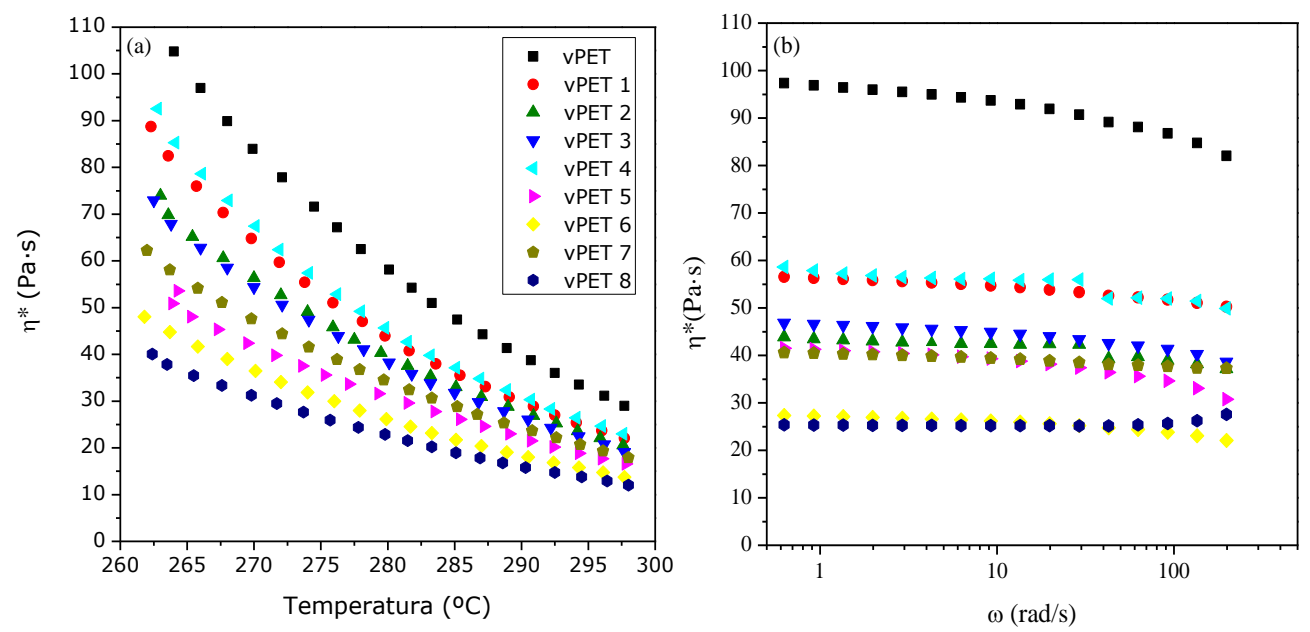

Figura 4.12 Barrido en temperatura (a) y en frecuencia (b) de las muestras VPET y VPET (1-8)

Todas las muestras presentaron una tendencia general donde la $\eta$ * disminuyó con el aumento de la temperatura debido a la degradación térmica (ver Figura 4.12 (a)). La disminución más significativa de $\eta^{*}$ se observó en las muestras de vPET $6(100 / 280 / 5)$ y vPET $8(300 / 280 / 5)$ las cuales corresponden a las referencias extruidas a mayor temperatura y menor caudal. Estos resultados nos sugieren que, a partir de estas condiciones de extrusión, se produce una mayor degradación termo-mecánica del PET, como ya fue discutido en la Figura 4.10. 
Por otro lado, se analizó el índice de fluidez (MFI), la viscosidad intrínseca $[\mu]$, y el peso molecular viscosimétrico (Mw) de las muestras vPET (1-8) (Tabla 4.6).

Tabla 4.6 Resultados de índice de fluidez, viscosidad intrínseca y peso molecular viscosimétrico de las muestras de VPET modificado termo-mecánicamente

\begin{tabular}{ccccc}
\hline $\begin{array}{c}\text { Condiciones } \\
\text { proceso }\end{array}$ & Muestra & MFI (g/10min) & $\begin{array}{c}{[\boldsymbol{\mu}]} \\
(\mathbf{d l} / \mathbf{g})\end{array}$ & Mw (g/mol) \\
\hline- & VPET & $44.6 \pm 1.5$ & 0.638 & 40706 \\
$100-265-5$ & VPET 1 & $53.4 \pm 2.0$ & 0.480 & 26787 \\
$100-265-9$ & VPET 2 & $46.8 \pm 6.4$ & 0.479 & 26705 \\
$300-265-5$ & VPET 3 & $51.8 \pm 1.7$ & 0.478 & 26623 \\
$300-265-9$ & VPET 4 & $55.3 \pm 0.7$ & 0.483 & 27034 \\
$100-280-9$ & VPET 5 & $58.3 \pm 2.3$ & 0.475 & 26378 \\
$100-280-5$ & VPET 6 & $74.7 \pm 4.5$ & 0.401 & 20562 \\
$300-280-9$ & VPET 7 & $60.7 \pm 3.2$ & 0.463 & 25404 \\
$300-280-5$ & VPET 8 & $95.2 \pm 4.5$ & 0.398 & 20337 \\
\hline
\end{tabular}

Al igual que se observó en la reometría dinámica, en la tabla se observa el aumento de fluidez de las muestras sometidas a la degradación termo-mecánica (VPET 1-8) respecto al PET sin extruir (VPET); especialmente en las muestras VPET 6 y VPET 8. A partir de los resultados de la viscosidad intrínseca se pudo determinar el peso molecular viscosimétrico. De esta manera, se pudo ver cómo al someter al VPET a la degradación termo-mecánica se produce la escisión de la cadena polimérica formando cadenas de menor peso molecular, especialmente, en los ciclos más severos de extrusión (VPET 6 y vPET 8).

Las propiedades reológicas de los polímeros dependen fuertemente de su estructura molecular, especialmente del peso molecular $(\mathrm{Mw})^{16}$ por lo que, cualquier cambio que se produzca en las condiciones de extrusión provoca la disminución de la viscosidad ([H] y MFI), y consecuentemente, los valores de $\mathrm{Mw}^{17,18}$. 


\section{Propiedades térmicas}

Para conocer el comportamiento térmico que tiene el VPET al ser sometido a la degradación termo-mecánica se analizaron por DSC las muestras VPET (1-8). En la Tabla 4.7 se muestran las propiedades térmicas y en la Figura 4.13 las endotermas y exotermas de dichas muestras.

Tabla 4.7 Propiedades térmicas de las muestras de vPET modificado termo-mecánicamente

\begin{tabular}{|c|c|c|c|c|c|}
\hline $\begin{array}{c}\text { Condiciones } \\
\text { proceso }\end{array}$ & Muestra & $\mathrm{T}_{\mathrm{g}} \pm 0.5\left({ }^{\circ} \mathrm{C}\right)$ & $T_{f} \pm 0.5\left({ }^{\circ} \mathrm{C}\right)$ & $T_{c} \pm 0.5\left({ }^{\circ} \mathrm{C}\right)$ & $X \pm 1(\%)$ \\
\hline- & VPET & 80.1 & 244.6 & 166.7 & 27 \\
\hline $100-265-5$ & VPET 1 & 78.6 & 231.1 & 196.3 & 29 \\
\hline $100-265-9$ & VPET 2 & 79.5 & 232.1 & 197.9 & 32 \\
\hline $300-265-5$ & VPET 3 & 79.6 & 232.3 & 198.5 & 28 \\
\hline $300-265-9$ & VPET 4 & 81.0 & 231.5 & 195.6 & 28 \\
\hline $100-280-9$ & VPET 5 & 78.5 & 232.4 & 197.5 & 30 \\
\hline $100-280-5$ & VPET 6 & 77.7 & 232.1 & 201.3 & 34 \\
\hline $300-280-9$ & VPET 7 & 80.1 & 232.3 & 200.4 & 30 \\
\hline $300-280-5$ & VPET 8 & 78.2 & 231.8 & 204.1 & 34 \\
\hline
\end{tabular}
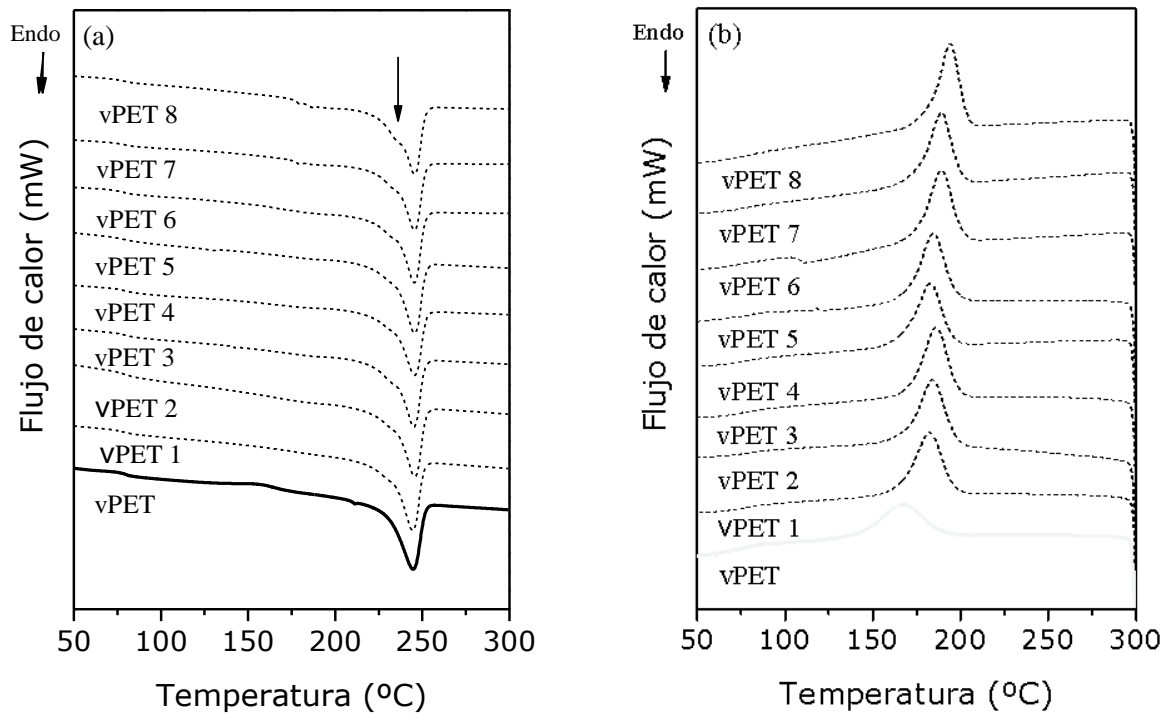

Figura 4.13 Curvas DSC de a) fusión y b) cristalización de las muestras de VPET modificado termomecánicamente 
Además del aumento de la fluidez por la disminución de peso molecular, estos resultados mostraron cómo la degradación termo-mecánica que sufre el vPET durante la extrusión, causa también cambios en la microestructura y en las propiedades térmicas, especialmente en la cristalinidad. Como se puede observar, las referencias que sufrieron mayor degradación térmica fueron, de nuevo, el vPET6 (100-280-5) y VPET8 (300-280-5) que correspondían con los ciclos de extrusión de mayor temperatura y menor caudal, presentando los valores de cristalinidad más altos (34\%). Este aumento de cristalinidad sugiere que la cadena polimérica del VPET tiene mayor capacidad de formar sistemas cristalinos con menos energía y de forma más ordenada, debido principalmente a que son cadenas de menor tamaño, como ya se demostró en los ensayos de Mw.

Si nos fijamos en la Figura 4.13 (b) se observa que la curva de enfriamiento del vPET muestra un pico de cristalización ancho, lo cual indica una velocidad de cristalización más lenta, característica de polímeros con mayor peso molecular. Al contrario, las muestras extruidas (VPET 1-8) tienen una exoterma bien definida, con un pico de cristalización agudo durante el enfriamiento. Este hecho se produce debido a la reducción de la longitud de la cadena del VPET con la extrusión, lo cual facilita el proceso de cristalización ${ }^{16}$. La rotura de las cadenas con la degradación termo-mecánica también afecta a la $T_{c}$, haciendo que las cadenas más cortas cristalicen antes (a una temperatura más alta).

El efecto de la degradación también puede verse en las curvas de fusión donde se puede observar cómo las muestras más degradadas, VPET 6 y vPET 8, presentan una endoterma de fusión más ancha, que incluso pueden diferenciar dos picos de fusión, característico de materiales de PET que han sufrido degradación y están formando nuevas poblaciones cristalinas de temperaturas de fusión más baja (Figura $4.13(\mathrm{a}))^{19}$.

\section{Propiedades mecánicas}

Se ha demostrado que estos tratamientos son capaces de disminuir la fluidez de las matrices estudiadas, a costa de perder peso molecular de una forma importante, influyendo a su vez en la población cristalina del polímero; por este motivo es de suma importancia, evaluar, también, la influencia de la degradación termo-mecánica en las propiedades mecánicas del VPET modificado. Las propiedades estudiadas fueron el Módulo de Young (Tabla 4.8) y el Impacto Charpy (Figura 4.14). 
Tabla 4.8 Módulo de Young de las muestras de VPET modificado termo-mecánicamente

\begin{tabular}{ccc}
\hline Muestra & $\begin{array}{c}\text { Condiciones } \\
\text { proceso }\end{array}$ & Módulo de Young \\
\hline VPET & - & $2396 \pm 36$ \\
VPET 1 & $100-265-5$ & $2375 \pm 15$ \\
VPET 2 & $100-265-9$ & $2336 \pm 35$ \\
VPET 3 & $300-265-5$ & $2403 \pm 47$ \\
VPET 4 & $300-265-9$ & $2412 \pm 25$ \\
VPET 5 & $100-280-9$ & $2398 \pm 31$ \\
VPET 6 & $100-280-5$ & $2380 \pm 26$ \\
VPET 7 & $300-280-9$ & $2421 \pm 50$ \\
VPET 8 & $300-280-5$ & $2422 \pm 85$ \\
\hline
\end{tabular}

Como se puede ver en la Tabla anterior, la degradación termo-mecánica, a pesar de ser influyente en la cristalinidad, no causó pérdidas significativas del valor del Módulo de Young en las muestras de VPET extruidas $( \pm 3,5 \%)$. Sin embargo, debido la escisión de las cadenas que experimenta el vPET con la degradación termo-mecánica era predecible la importante caída del valor de la tenacidad de las muestras extruidas VPET 1-8 (Figura 4.14).

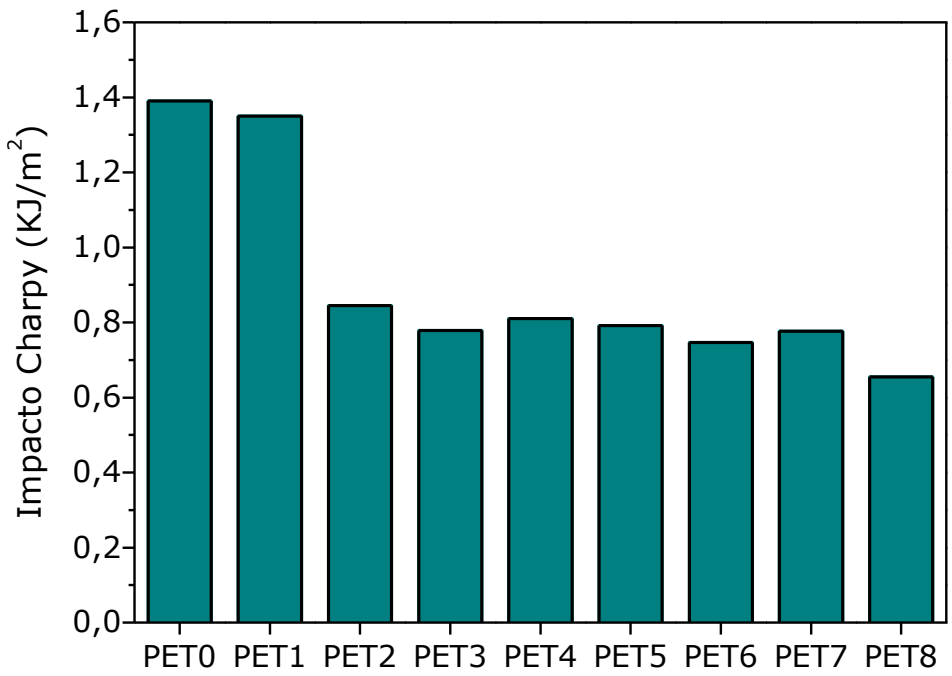

Figura 4.14 Impacto Charpy de las muestras de vPET modificado termo-mecánicamente

Las muestras sometidas a los ciclos de extrusión más severos (VPET 6 y vPET 8) presentaron una mayor pérdida de tenacidad (una caída aproximadamente del $50 \%$ ). Estas muestras, como se vio anteriormente, presentaron los valores más altos de cristalización, lo cual provoca la fragilización del VPET, sin convertirse en un importe efecto rigidizador que aumentará el Módulo. 
Así mismo, la muestra VPET 1, que corresponde con el PET extruido a las condiciones que se seleccionaron del DOE (100-265-5) presentó la menor pérdida de tenacidad y a la vez, importantes caídas de la viscosidad. Este resultado confirma la elección de estas condiciones de extrusión para la elaboración de las modificaciones física y química que se detallan más adelante.

\subsubsection{Degradación hidrolítica del PET virgen}

No solo el efecto de la cizalla mecánica y la temperatura son importantes en la degradación del PET, otro fenómeno importante que debe tenerse en cuenta durante el procesado es su carácter higroscópico. Con el fin de estudiar la degradación en presencia de humedad, se sometió al VPET a un proceso de extrusión, dosificando agua a través de una bomba peristáltica como se muestra en la Figura 4.15.

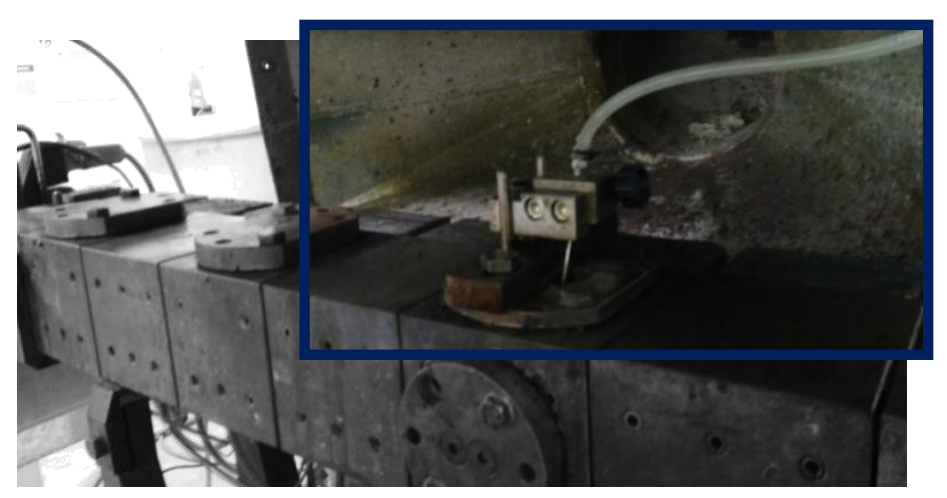

Figura 4.15 Extrusora con bomba peristáltica utilizada para la degradación hidrolítica

Las tasas de hidrólisis y la degradación térmica se estudiaron en función de la cantidad de agua (flujo volumétrico) y de la temperatura, respectivamente. El máximo flujo volumétrico que admitió la bomba peristáltica fue de un $22 \%$ de agua que corresponde a $18 \mathrm{ml} / \mathrm{min}$ y la mínima de $9 \%$ ( $9 \mathrm{ml} / \mathrm{min})$. La temperatura más baja se estableció a $265^{\circ} \mathrm{C}$ y la más alta $280^{\circ} \mathrm{C}$. Se fijaron los parámetros de extrusión seleccionados a partir de los resultados del DOE (sección 4.2.1) con variables fijas como velocidad de husillo $(100 \mathrm{rpm})$ y caudal $(5 \mathrm{~kg} / \mathrm{h})$.

En la Tabla 4.9 se pueden observar las muestras sometidas a la degradación hidrolítica y las condiciones de extrusión a las que fueron obtenidas. Las muestras son nominadas VPET (A-D) por el orden de: cantidad de agua/temperatura de procesado. 
Tabla 4.9 Composición y condiciones de procesado de degradación hidrolítica de VPET

\begin{tabular}{cccc}
\hline $\begin{array}{c}\text { Muestra: cantidad de agua/ } \\
\text { temperatura }\end{array}$ & $\mathbf{V}_{\mathbf{H 2 O}}(\mathbf{m l} / \mathbf{m i n})$ & \% Agua & T( $\left.{ }^{\circ} \mathrm{C}\right)$ \\
\hline PET A: $22 \% / 265^{\circ} \mathrm{C}$ & 18 & $22 \%$ & 265 \\
PET B: $22 \% / 280^{\circ} \mathrm{C}$ & 18 & $22 \%$ & 280 \\
PET C: $9 \% / 265^{\circ} \mathrm{C}$ & 9 & $9 \%$ & 265 \\
PET D: $9 \% / 280^{\circ} \mathrm{C}$ & 9 & $9 \%$ & 280 \\
\hline
\end{tabular}

\subsubsection{Resultados de la degradación hidrolítica del PET virgen}

En esta sección se muestra la caracterización que se llevó a cabo de las muestras sometidas a la degradación hidrolítica (vPET A-D).

\section{Análisis reológico}

En primer lugar, se evaluó el efecto de la degradación hidrolítica del VPET sobre la viscosidad mediante reometría dinámica. En la Figura 4.16 se muestra la viscosidad compleja $\left(n^{*}\right)$ frente al barrido en temperatura y en frecuencia del VPET y de las muestras sometidas a la degradación hidrolítica (VPET A-D). Tras la reometría dinámica se analizó el índice de fluidez (MFI), la viscosidad intrínseca $[\mu]$, y el peso molecular viscosimétrico ( $\mathrm{Mw}$ ) de estas muestras (Tabla 4.10).
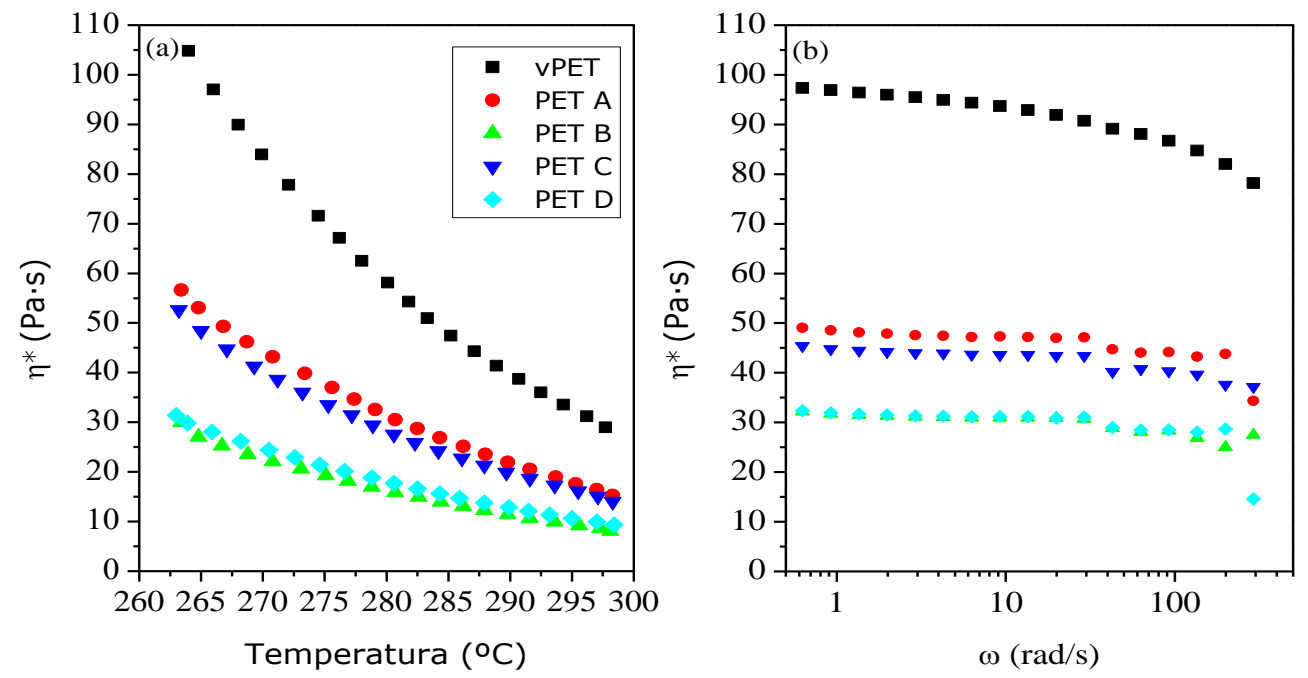

Figura 4.16 Viscosidad compleja frente a temperatura (a) y frecuencia (b) de las muestras del vPET modificadas hidrolíticamente 
Como era de esperar, todas las referencias sometidas a los ciclos de extrusión hidrolíticos ( $v P E T$ A-D) presentaron valores de $\eta^{*}$ más bajos que el VPET sin extruir. En la Figura 4.16 se puede observar cómo existen dos poblaciones de muestras con valores de $\eta^{*}$ diferenciados. Las muestras PET A $\left(22 \% / 265^{\circ} \mathrm{C}\right)$ y PET C $\left(9 \% / 265^{\circ} \mathrm{C}\right)$, extruidas a menor temperatura $\left(265^{\circ} \mathrm{C}\right)$, presentaron los valores de viscosidad más altos. En cambio, PET B $\left(22 \% / 280^{\circ} \mathrm{C}\right)$ y PET D $\left(9 \% / 280^{\circ} \mathrm{C}\right)$, extruidas a mayor temperatura $\left(280^{\circ} \mathrm{C}\right)$, tienen rangos de viscosidad menores. De estos resultados se pudo determinar que el factor temperatura (degradación térmica) influye más que la cantidad de agua adicionada (degradación hidrolítica) en la disminución de $\eta^{*}$ de las muestras estudiadas.

Esto también se puede ver en la Tabla 4.10, donde se muestran los valores de índice de fluidez (MFI), viscosidad intrínseca $[\mu]$, así como el peso molecular viscosimétrico $(\mathrm{Mw})$. Los valores de MFI de PET A y PET C presentaron valores similares al VPET 1 (53 g/10min) que fue extruido a las mismas condiciones, pero sin contenido de agua. La disminución de los pesos moleculares en las muestras PET B y PET C $\left(280^{\circ} \mathrm{C}\right)$ respecto a las muestras PET A y PET C $\left(265^{\circ} \mathrm{C}\right)$ confirmaron la escisión de las cadenas poliméricas por la degradación térmica.

Tabla 4.10 Viscosidad intrínseca, índice de fluidez y peso molecular de las muestras de vPET modificado hidrolíticamente

\begin{tabular}{ccccccc}
\hline Propiedad & vPET & vPET1 & $\begin{array}{c}\text { PET A } \\
22 \% / 265^{\circ} \mathrm{C}\end{array}$ & $\begin{array}{c}\text { PET B } \\
22 \% / 280^{\circ} \mathrm{C}\end{array}$ & $\begin{array}{c}\text { PET C } \\
9 \% / 265^{\circ} \mathrm{C}\end{array}$ & $\begin{array}{c}\text { PET D } \\
9 \% / 280^{\circ} \mathrm{C}\end{array}$ \\
\hline MFI & 44.6 & 53.4 & 49.9 & 77.5 & 57.7 & 73.2 \\
$(\mathbf{g} / \mathbf{1 0 m i n})$ & \pm 1.5 & \pm 2.0 & \pm 1.2 & \pm 1.2 & \pm 1.9 & \pm 1.7 \\
[ $\mathbf{\mu}]$ & 0.638 & 0.480 & 0.475 & 0.459 & 0.471 & 0.464 \\
$(\mathbf{d L} / \mathbf{g})$ & \pm 0.03 & \pm 0.03 & \pm 0.03 & \pm 0.02 & \pm 0.04 & \pm 0.02 \\
$\mathbf{M w}$ & & & & & 26052 & 25484 \\
$(\mathbf{g} / \mathbf{m o l})$ & 40706 & 26787 & 26378 & 25082 & 26052 \\
\hline
\end{tabular}

\section{Propiedades térmicas}

A continuación, para poder evaluar los cambios térmicos del VPET, al ser sometido a la degradación hidrolítica, se caracterizaron térmicamente por DSC las muestras de VPET (A-D). En la Tabla 4.11 se muestran los valores de las propiedades térmicas del vPET sin extruir, el VPET 1 extruido a las condiciones seleccionadas a partir del DOE (100-265-5) y las muestras sometidas a la degradación hidrolítica vPET (A-D). 
Tabla 4.11 Propiedades térmicas de las muestras de VPET modificado hidrolíticamente

\begin{tabular}{ccccc}
\hline Muestra & $\mathbf{T}_{\mathbf{g}} \mathbf{\pm 0 . 5}\left({ }^{\circ} \mathbf{C}\right)$ & $\mathbf{T}_{\mathbf{f}} \mathbf{0 . 5}\left({ }^{\circ} \mathbf{C}\right)$ & $\mathbf{T}_{\mathbf{c}} \mathbf{\pm 0 . 5}\left({ }^{\circ} \mathbf{C}\right)$ & $\mathbf{X \pm \mathbf { 1 } ( \% )}$ \\
\hline VPET & 80.1 & 244.6 & 166.7 & 27 \\
VPET 1 & 78.6 & 231.1 & 196.3 & 29 \\
PET A: $22 \% / 265^{\circ} \mathrm{C}$ & 79.0 & 231.9 & 196.4 & 29 \\
PET B: $22 \% / 280^{\circ} \mathrm{C}$ & 78.5 & 232.5 & 195.4 & 30 \\
PET C: $9 \% / 265^{\circ} \mathrm{C}$ & 80.8 & 232.0 & 194.9 & 31 \\
PET D: $9 \% / 280^{\circ} \mathrm{C}$ & 85.8 & 232.2 & 201.1 & 33 \\
\hline
\end{tabular}

Como se vio anteriormente, el efecto de la temperatura predominó sobre la cantidad de agua en la disminución de la viscosidad. Por lo que, al igual que ocurrió en la degradación termo-mecánica, los valores de la Tf deberán disminuir respecto a vPET en las muestras extruidas.

Las diferencias más significativas en las propiedades térmicas surgen en la $T_{c}$. En la curva de cristalización (Figura 4.17) se puede observar cómo las muestras de VPET (A-D) presentan un pico de cristalización más estrecho y a una temperatura de cristalización mayor que el VPET. Estas variaciones térmicas observadas podrían ser atribuidas a la disminución del peso molecular (ver Tabla 4.10) y al posible cambio en su distribución ya que, una consecuencia directa de la reducción del Mw en polímeros es el aumento de la velocidad de cristalización que corresponden con temperaturas más altas de cristalización ${ }^{20}$.
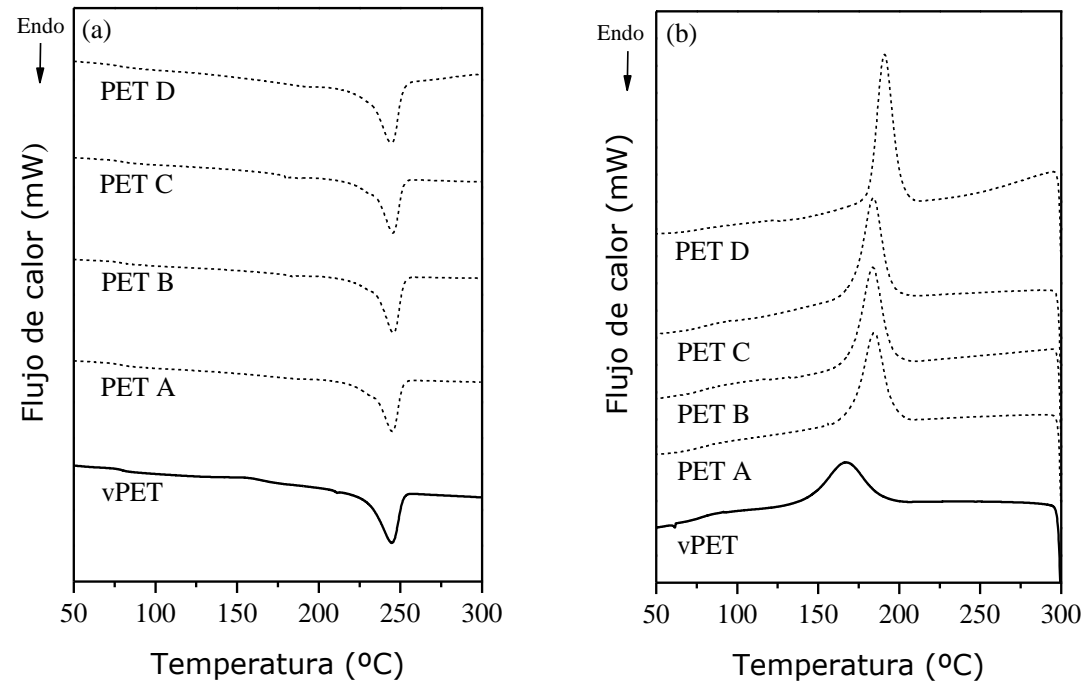

Figura 4.17 Curvas DSC de a) fusión y b) cristalización de las muestras de VPET modificado hidrolíticamente 


\section{Propiedades mecánicas}

Finalmente, se evaluaron el Módulo de Young y el Impacto Charpy de las muestras modificadas hidrolíticamente (PET A-D), del vPET sin extruir y del vPET 1 (extruido a las mismas condiciones sin agua).

Tabla 4.12 Valores de Módulo de Young de las muestras de VPET modificado hidrolíticamente

\begin{tabular}{ccc}
\hline Muestra & Condiciones proceso & Módulo de Young \\
\hline VPET & - & $2396 \pm 36$ \\
VPET 1 & $0 \% / 265^{\circ} \mathrm{C}$ & $2375 \pm 15$ \\
PET A & $22 \% / 265^{\circ} \mathrm{C}$ & $2342 \pm 17$ \\
PET B & $22 \% / 280^{\circ} \mathrm{C}$ & $2368 \pm 11$ \\
PET C & $9 \% / 265^{\circ} \mathrm{C}$ & $2420 \pm 57$ \\
PET D & $9 \% / 280^{\circ} \mathrm{C}$ & $2448 \pm 103$ \\
\hline
\end{tabular}

Como se puede ver en la Tabla anterior, los resultados mostraron que los ciclos hidrolíticos sobre el VPET no provocaron cambios significativos en el Módulo de Young. Sin embargo, del mismo modo que ocurrió en la degradación termomecánica, el Impacto Charpy se ve afectado negativamente y en cuantía importante.

En la Figura 4.18 se muestran los resultados de tenacidad, donde se observa la disminución de su valor en las muestras sometidas a la degradación hidrolítica. Esto es debido al acortamiento de las cadenas que sufre el PET con la degradación hidrolítica haciendo que los materiales sean más frágiles.

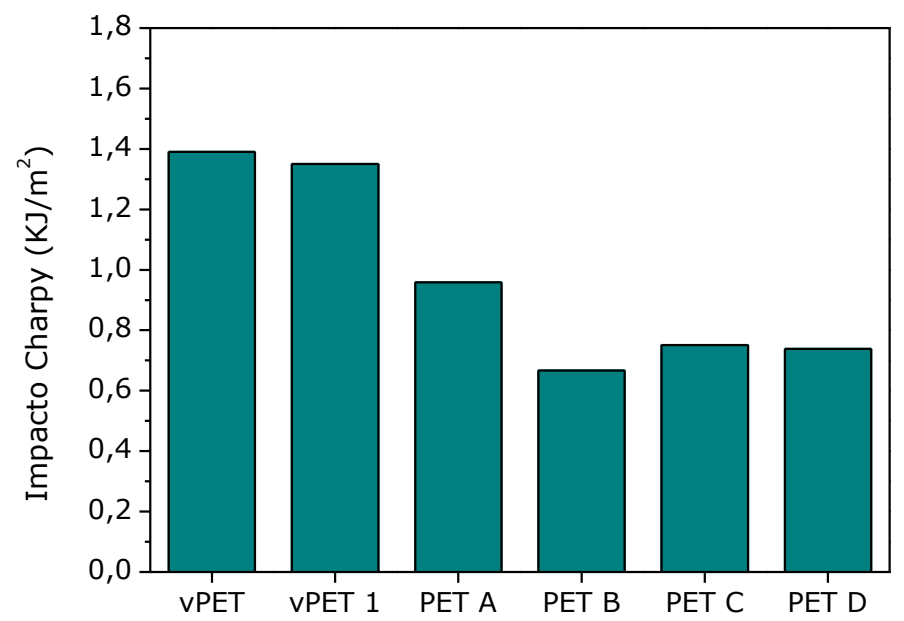

Figura 4.18 Impacto Charpy de las muestras de VPET modificado hidrolíticamente 
Finalmente, la degradación hidrolítica parece tener un efecto menos importante en la microestructura del PET, durante su procesado, que la temperatura; siendo esta última un factor catalizador para que la hidrólisis ocurra. $\mathrm{Ni}$ en las condiciones más extremas de hidrólisis estudiadas (altos porcentajes de agua y altas temperatura, muestra B) se alcanza la pérdida tan importante de $\mathrm{Mw}$ y tenacidad que se consiguen con el efecto de condiciones extremas de termo-cizalla.

\subsubsection{Modificación química del PET virgen}

El método de modificación química se llevó a cabo mediante el proceso de extrusión reactiva. La extrusión reactiva tiene lugar dentro de la línea de extrusión en la que se da la reacción química de los materiales, durante el proceso de extrusión en continuo del polímero. La extrusión reactiva se ha utilizado como un método eficiente para la polimerización de monómeros así como para la modificación química de los mismos ${ }^{21}$.

La metodología de modificación química, propuesta en esta investigación, utiliza la extrusión reactiva y la funcionalización, a partir de la introducción de grupos funcionales dentro de la cadena principal, en los grupos finales o cadenas laterales, o a partir de la modificación de los grupos funcionales existentes. En este sentido, a partir de la utilización de aditivos como el ácido 5 amino isoftálico capaces de actuar como núcleos ramificados, se pueden producir grandes variaciones en la viscosidad de los materiales ${ }^{21}$, porque se cambia la configuración espacial y las ramificaciones del mismo.

La mayor importancia científica y tecnológica de este estudio radica en el análisis de los cambios en la estructura molecular que experimenta el VPET con la modificación química, los cuales tienen una gran influencia en las propiedades reológicas.

Para entender el efecto que tiene el aditivo en la estructura y en las propiedades del VPET se estudiaron diferentes concentraciones, variando la cantidad de aditivo desde $0.25 \%$ hasta un $1 \%$ en peso (Tabla 4.13). La temperatura de extrusión fue de $265^{\circ} \mathrm{C}$, la velocidad de husillo de $100 \mathrm{rpm}$ y el caudal de $5 \mathrm{Kg} / \mathrm{h}$ (condiciones seleccionadas a partir de los resultados del DOE llevado a cabo en la sección 4.2.1). 
Tabla 4.13 Composición de las muestras de vPET modificadas químicamente

\begin{tabular}{cc}
\hline Muestra & Aditivo (wt \%) \\
\hline vPET & - \\
vPET 0.25\% & $0.25 \%$ \\
vPET 0.5\% & $0.5 \%$ \\
vPET 1\% & $1 \%$ \\
\hline
\end{tabular}

\subsubsection{Resultados de la modificación química del PET virgen}

Una vez se llevó a cabo la modificación química del vPET mediante la extrusión reactiva, se caracterizaron las muestras obtenidas en términos reológicos, térmicos, mecánicos y morfológicos.

\section{Análisis reológico}

La caracterización reológica de vPET modificado químicamente se llevó a cabo por reometría dinámica. En la Figura 4.19 se muestra el efecto de la variación de la concentración del aditivo sobre la viscosidad compleja ( $\left.\eta^{*}\right)$ en función de la temperatura. En la gráfica se representan dos flechas discontinuas; una de las cuales (horizontal) señala el límite de los valores de $\eta^{*}$ que son requeridos para poder utilizar estos materiales en el procesado de composites por pultrusión. Un límite importante que deben cumplir los materiales es que no tengan una viscosidad menor de 25Pa.s, debido a que el procesado de materiales con esta viscosidad, hace imposible su manejabilidad y la producción de composites por pultrusión. La otra flecha (vertical), señala la temperatura que se va a utilizar en la fabricación de los composites por pultrusión, para de esta manera, conocer el punto exacto de la curva de viscosidad en las condiciones de proceso buscadas. Hasta ahora, todos los materiales modificados en la sección 4.2.1 y 4.2.2, vía termo-mecánica e hidrolíticamente, tienen viscosidades muy por encima de $25 \mathrm{~Pa} \cdot \mathrm{s}$ (ver Figuras 4.12 y 4.16, respectivamente). Esto demuestra la gran influencia del aditivo en la viscosidad, por encima de cualquier parámetro experimental hasta ahora estudiado.

También es importante mencionar que las muestras de VPET modificadas químicamente se comparan con el vPET1 (extruido de la experimentación 4.2.1) para poder conocer con exactitud el efecto del aditivo en las propiedades del PET, sin tener en cuenta el efecto de la extrusión que se analizó en las secciones anteriores. 


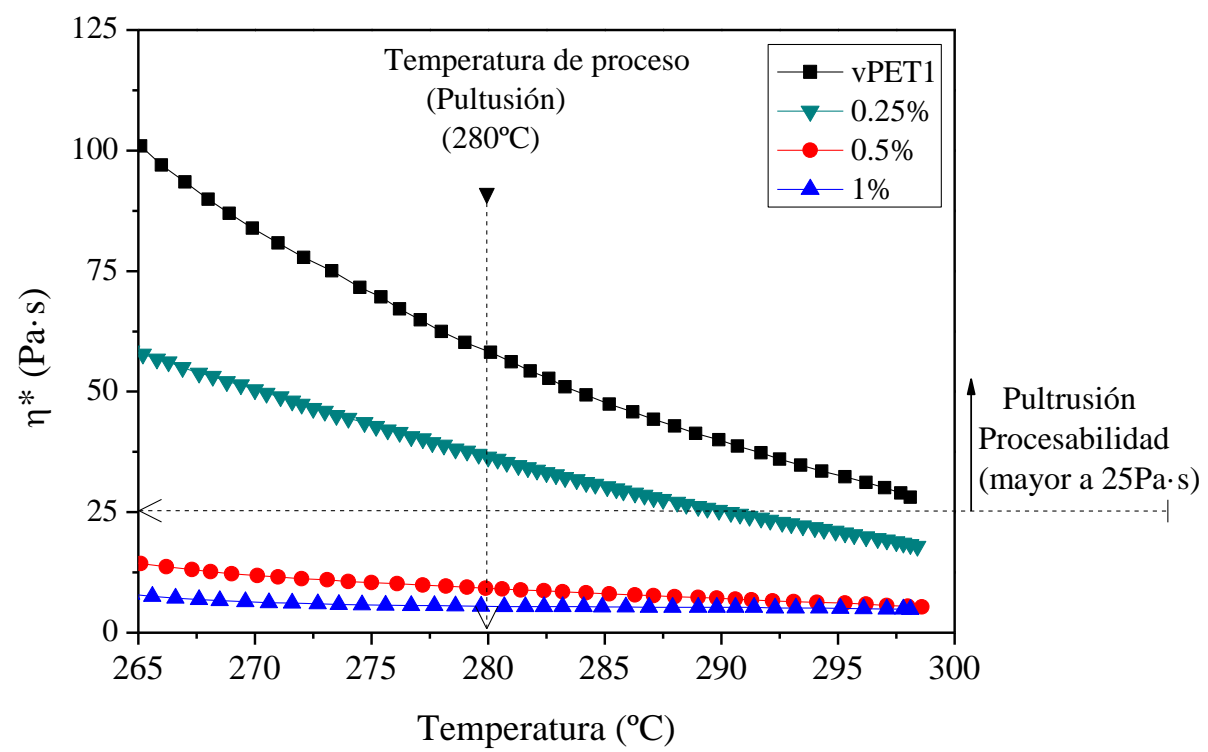

Figura 4.19 Viscosidad compleja frente a temperatura de VPET con diferentes concentraciones de aditivo

En el gráfico se puede apreciar cómo el aditivo produce una reducción de los valores de la viscosidad $\left(n^{*}\right)$, siendo más pronunciada a medida que se aumenta la concentración del aditivo. Estos resultados confirmaron la utilización del método químico como una técnica efectiva para la disminución de la viscosidad del PET.

A partir de estos resultados, se comprobó que concentraciones de aditivo superiores a $0.25 \%$ provocan una disminución de la viscosidad por debajo del límite establecido. Con lo cual, la concentración de $0.25 \%$ de aditivo fue seleccionada para la modificación química que se llevó a cabo posteriormente en las mezclas, ya que se encuentra dentro de los límites marcados para el procesado de los composites por pultrusión.

Posteriormente, estas muestras fueron analizadas por GPC. En la Figura 4.20 se muestran los valores de los pesos moleculares ( $\mathrm{Mw}$ ) obtenidos, a partir de los cuales se pudo observar que la disminución de la $n^{*}$ al incorporar el aditivo está asociada a una disminución del peso molecular (Mw) del sistema polimérico. Una vez que el VPET sin procesar es extruido (VPET $\rightarrow$ VPET1), se produce una disminución del peso molecular por la degradación termo-mecánica anteriormente estudiada. Del mismo modo, incorporando el aditivo con las mismas condiciones de extrusión, el peso molecular disminuye a medida que aumenta la concentración. De estos resultados se puede extraer que la pérdida de $\mathrm{Mw}$ es aditiva tanto por el procesado como por el efecto del injerto químico. 


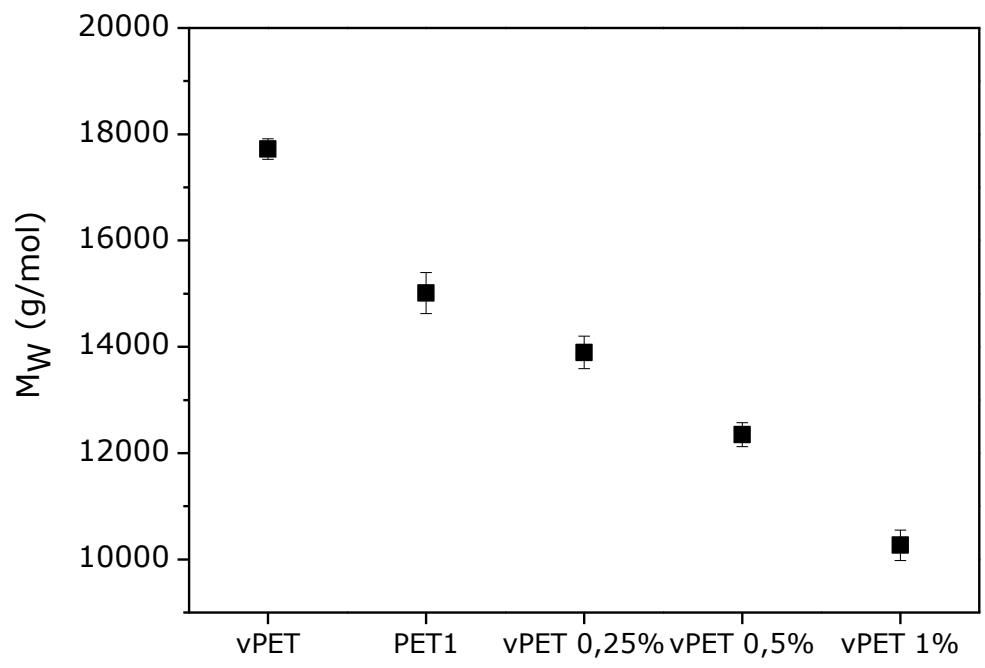

Figura 4.20 Peso molecular de VPET con diferentes concentraciones de aditivo calculado por GPC

\section{Propiedades térmicas}

Posteriormente, se realizó el análisis térmico de la muestra de VPET modificada químicamente. En la Figura 4.21, se muestran las curvas de fusión y cristalización del vPET modificado químicamente con las distintas concentraciones de aditivo. A continuación, en la Tabla 4.14, se muestran los valores cuantitativos de las propiedades térmicas obtenidas de las curvas DSC.
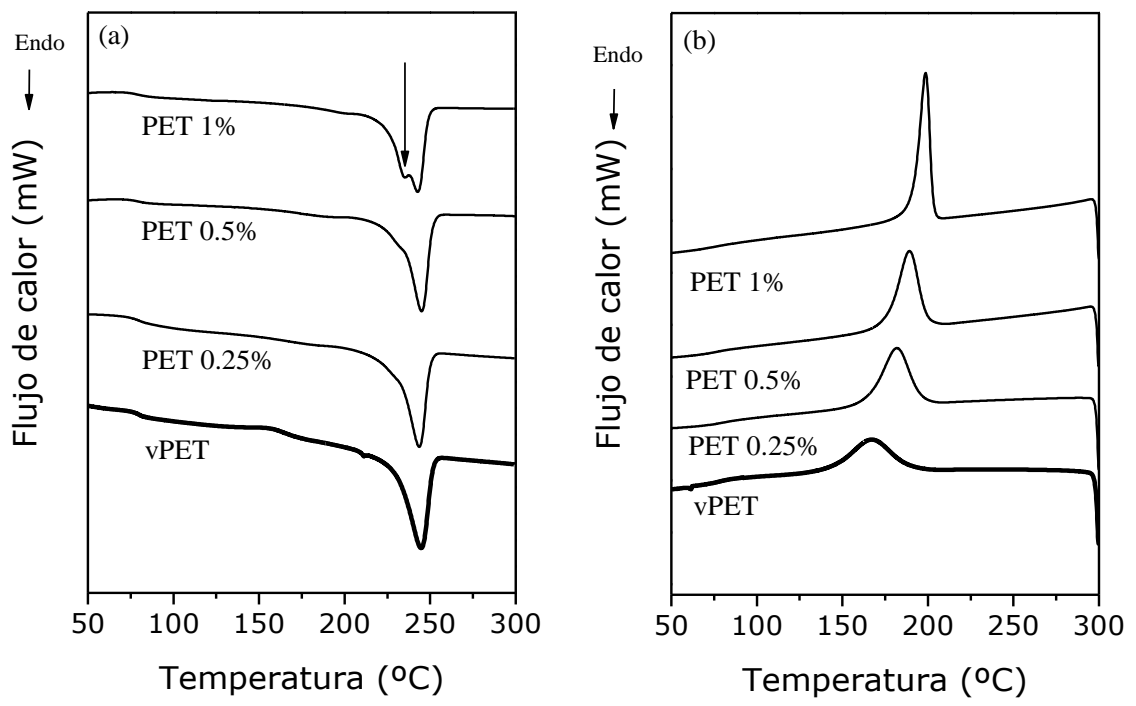

Figura 4.21 Curvas DSC a) Fusión b) Cristalización de vPET modificado químicamente 
En las curvas de fusión (Figura 4.21 (a)) se observa cómo a medida que aumenta la cantidad de aditivo aparece un segundo pico de fusión a una temperatura más baja. Este pico es más pronunciado cuanto mayor es la cantidad de aditivo (PET 1\%), lo cual indica que el aditivo promueve las escisiones de las cadenas poliméricas formando dos secciones cristalinas diferenciadas en el mismo pico endotérmico.

Estos picos de fusión múltiple, observados durante el escaneo de calentamiento DSC, son atribuibles a la distribución de cristales con diferente espesor laminar y a la fusión de diferentes estructuras cristalinas ${ }^{7}$. El hecho de que el pico de fusión aparezca a más bajas temperaturas, sugiere la formación de una estructura cristalina de menor tamaño, que aparece debido a cadenas más cortas con mayor capacidad de empaquetarse. En cambio, el segundo pico endotérmico que se observa en la curva de fusión, no muestra cambios significativos cuando la cantidad de aditivo aumenta (variación de $\pm 2{ }^{\circ} \mathrm{C}$ ), lo cual nos indica que la estructura cristalina mayoritaria prácticamente se mantiene.

Tabla 4.14 Composición y propiedades térmicas de vPET con distinta concentración de aditivo

\begin{tabular}{|c|c|c|c|c|}
\hline Muestra & $\mathrm{T}_{\mathrm{g}} \pm 0.5^{\circ} \mathrm{C}$ & $T_{f} \pm 0.5^{\circ} \mathrm{C}$ & $T_{c} \pm 0.5^{\circ} \mathrm{C}$ & $x \pm 1 \%$ \\
\hline VPET & 80.1 & 244.6 & 166.7 & 27 \\
\hline vPET 1 & 78.6 & 231.1 & 196.3 & 29 \\
\hline vPET $0.25 \%$ & 79.6 & 243.6 & 181.6 & 27 \\
\hline VPET $0.5 \%$ & 79.6 & 245.0 & 189.1 & 28 \\
\hline VPET $1 \%$ & 79.5 & 242.7 & 198.7 & 29 \\
\hline
\end{tabular}

En las curvas de cristalización (Figura 4.21 (b)) se puede observar que a medida que se aumenta la cantidad de aditivo, los picos de cristalización son más estrechos y la cristalización comienza a una $T_{c}$ más alta. El cambio a $T_{c}$ más altas y picos más estrechos nos indica que el proceso de cristalización de las muestras modificadas químicamente se produce antes que el de VPET puro, es decir, presentan mayor capacidad de cristalización, al igual que todas las muestras modificadas hasta ahora ${ }^{14}$. El porcentaje de cristalinidad no presentó cambios significativos, lo cual indica que se tiene la misma cantidad de cristales pero de diferentes tamaños.

\section{Propiedades mecánicas}

Por último, se analizaron los módulos elásticos y la tenacidad de las muestras de VPET sometidas a la modificación química. En la Figura 4.22 se muestran los valores de Módulo de Young del VPET y las muestras del VPET modificadas químicamente con diferentes concentraciones de aditivo. 


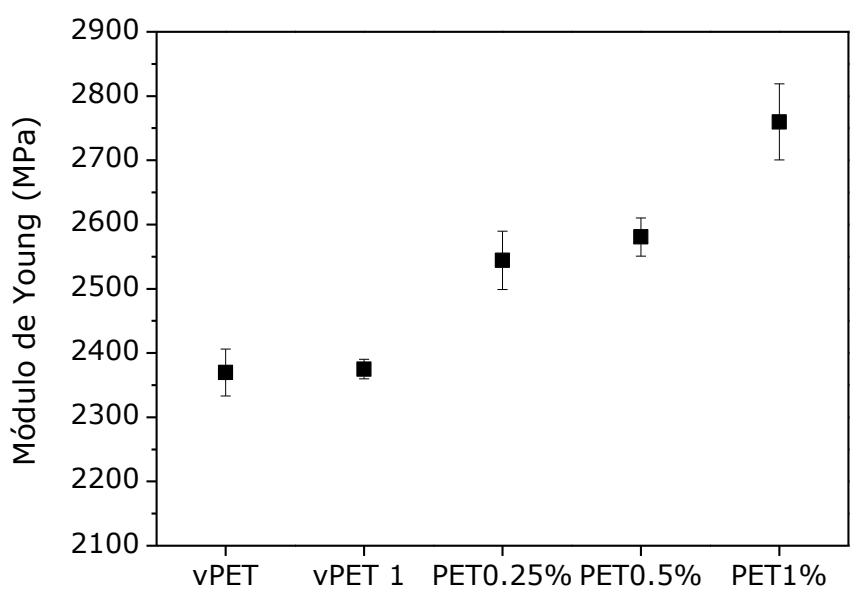

Figura 4.22 Módulo de Young de VPET modificado químicamente con diferentes concentraciones de aditivo

Como se puede observar, la incorporación del aditivo en la matriz de VPET provoca un aumento significativo del Módulo de Young. Esto puede ser atribuido a que, como se explicó anteriormente en la Figura 4.9 (reacciones teóricas del aditivo), el aditivo se inserta en la cadena polimérica introduciendo grupos aromáticos que proporcionan más rigidez al material y promueven, con ello, el incremento en los valores de Módulo de Young. Por otra parte, esta importante rigidización, unida a la pérdida de $\mathrm{Mw}$, trae como consecuencia una significativa caída de tenacidad, que se observa en la Figura 4.23 y que debe ser solventada, para considerar validos estos materiales modificados.

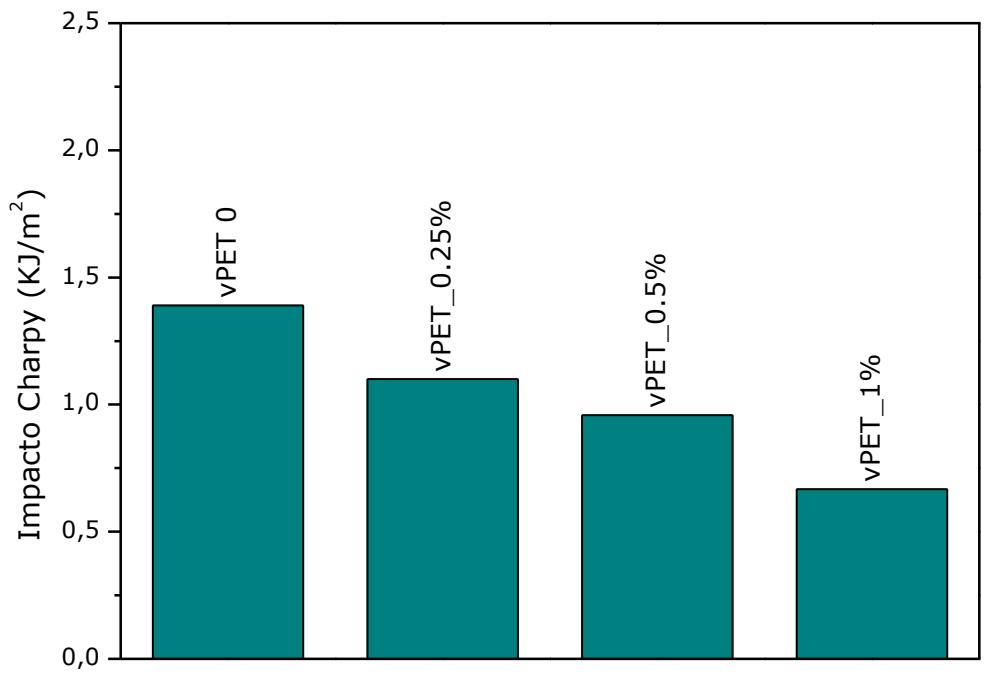

Figura 4.23 Impacto Charpy de vPET modificado químicamente con diferentes concentraciones de aditivo 


\section{Análisis espectroscópico}

Con el fin de estudiar el efecto que tiene el aditivo en la estructura molecular del VPET, se llevó a cabo un análisis de los espectros IR del PET virgen sin procesar (VPET) y el PET modificado con una cantidad muy elevada de aditivo (VPET 20\%). Esta cantidad no interesa para la modificación, por el espectacular efecto sobre la reducción de la viscosidad, pero es importante para detectar cambios en los espectros IR.

Normalmente, para estudiar la degradación del PET con los ciclos de procesado se toma la señal de $1400 \mathrm{~cm}^{-1}$ como banda de normalización ${ }^{22}$, que corresponde con los modos de vibración del anillo bencénico. En este caso, no podemos utilizar esta señal como banda de normalización, ya que tanto el vPET como el aditivo cuentan con modos de vibración similares, debido a los grupos funcionales que tienen en común: anillos bencénicos y grupos carboxílicos, por lo que la intensidad de esta banda no se mantendría constante. Por este motivo, la elección de la banda de normalización para este trabajo se basó en el estudio de los grupos funcionales no comunes en ambos compuestos y los menos susceptibles a cambios de estructura por la presencia del aditivo. Como resultado, se eligió como banda de normalización la correspondiente a la vibración del $\mathrm{CH}_{2}$ a $1340 \mathrm{~cm}^{-1}$. En la Figura 4.24 se muestra el espectro IR del vPET y del VPET $20 \%$ $\left(600 \mathrm{~cm}^{-1}-1800 \mathrm{~cm}^{-1}\right)$.

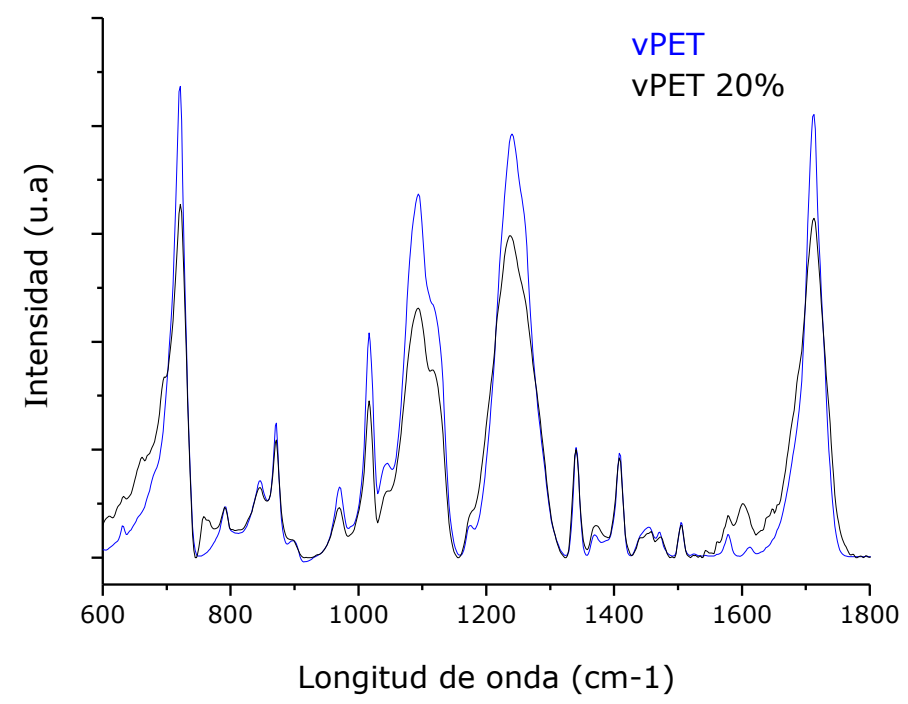

Figura 4.24 Espectros FTIR de vPET, vPET $20 \%$ y el aditivo aislado $\left(600-1800 \mathrm{~cm}^{-1}\right)$ 
De acuerdo con el esquema de las reacciones de degradación que sufre el PET (explicado anteriormente en la Figura 4.9), los enlaces susceptibles a la rotura son enlaces $\mathrm{O}-\mathrm{CH}_{2}$, que generan grupos terminales - $\mathrm{OH}$ con su ruptura. Por ello, el aumento de estos grupos hidroxilo - $\mathrm{OH}$ es una evidencia de la disminución de viscosidad de estos materiales ${ }^{23}$.

Por este motivo, si se observa la Figura 4.24, se puede ver la evidencia de la rotura de este enlace en la disminución de la intensidad de la banda de vibración correspondiente $\mathrm{O}-\mathrm{CH}_{2}$ a $1116 \mathrm{~cm}^{-1}$. Como se ha explicado anteriormente, la rotura de estos enlaces genera grupos hidroxilo $-\mathrm{OH}$, por lo que, otra confirmación de la escisión de la cadena la podemos ver en la Figura 4.25, donde los grupos -OH libres se evidencian por la aparición de picos entre 3500 y 3700 $\mathrm{cm}^{-1}$.

Cabe recordar, que otro de los grupos que se genera con la degradación térmica son grupos vinil éster. Estos grupos se pueden ver en los espectros a través de los enlaces alquenos que presentan sus bandas características $\mathrm{C}=\mathrm{C}$ en $1600 \mathrm{~cm}^{-1}$. Sin embargo, estos grupos generados de éster de vinilo no son responsables de la disminución del viscosidad del material, sino del cambio de color (amarillento) que se pudo ver en las muestras modificadas químicamente ${ }^{23}$.

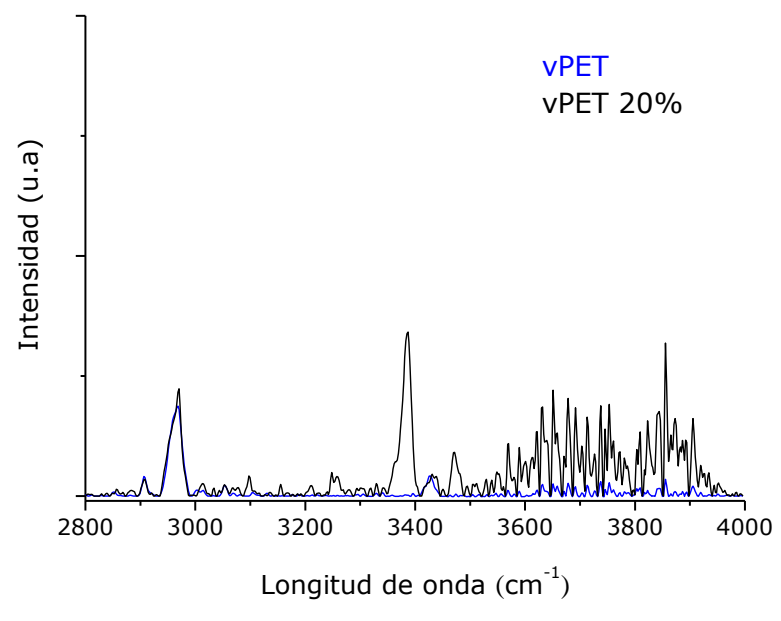

Figura 4.25 Espectro de vPET y vPET $20 \%\left(2800-4000 \mathrm{~cm}^{-1}\right)$

Por otra parte, una manera de confirmar la presencia del aditivo consistió en observar señales características del mismo que no están presentes en el VPET, como es el grupo amino que es evidenciado por las bandas de $3388 \mathrm{~cm}^{-1}$ y 750 $\mathrm{cm}^{-1}$. 
De las posibles reacciones del aditivo con el PET, también se planteó la formación de grupos anhídridos formados por la reacción entre los grupos carboxílicos del aditivo y los grupos carboxílicos terminales (que se generan por la degradación térmica e hidrolítica). Sin embargo, las bandas del enlace $\mathrm{C}=\mathrm{O}$ del grupo anhídrido pueden verse enmascaradas, debido a la baja cantidad de aditivo que se incorpora, en comparación con la mayor cantidad de enlaces $\mathrm{C}=\mathrm{O}$ que están presentes en la cadena polimérica del PET $^{9}$.

Finalmente, la modificación química vía injerto del aditivo, por extrusión reactiva, resulta una forma efectiva de disminuir la viscosidad del VPET, usando cantidades muy pequeñas del aditivo. Las caídas de Mw pueden llegar a ser tan drásticas que pueden estar muy por debajo de la viscosidad que necesitan los procesos de obtención de composites. Además del efecto de la adición del aditivo, se suma el efecto propio de la termo-cizalla del procesado; haciendo más crítica aún la pérdida de viscosidad. Por este motivo, las siguientes modificaciones propuestas se hacen sobre mezclas poliméricas con rPET (mayor viscosidad de partida) para llegar a propiedades de compromiso.

\subsubsection{Modificación física y modificación química de las mezclas}

En esta sección se expone el procedimiento experimental que se llevó a cabo para la realización de la modificación reológica (disminución de la viscosidad) del rPET por el método físico y químico.

En primer lugar se realizó la modificación por el método físico mediante las mezclas de rPET blue con el PET virgen de alta fluidez (denominadas rPET:VPET). La temperatura de extrusión fue de $265^{\circ} \mathrm{C}$, la velocidad de husillo de $100 \mathrm{rpm}$ y el caudal de $5 \mathrm{~kg} / \mathrm{h}$ (condiciones seleccionadas a partir del DOE en la sección 4.2.1).

Posteriormente, con las mismas condiciones de extrusión, se llevó a cabo la modificación por el método químico de los polímeros puros (rPET y vPET) y de sus mezclas. Este proceso de modificación evalúa el efecto del aditivo y de las mezclas conjuntamente. Los polímeros se adicionaron junto al aditivo por la cámara más lejana a la boca o salida de la extrusora para permitir el mayor tiempo de reacción.

En la Tabla 4.15 se muestran las composiciones de las mezclas rPET:vPET (método de modificación físico) y las composiciones y cantidad de aditivo que se utilizaron en la modificación química. 
Tabla 4.15 Muestras modificadas física y químicamente

\begin{tabular}{|c|c|c|c|}
\hline Método & Muestra & $\begin{array}{l}\text { rPET:vPET } \\
\text { (wt:wt\%) }\end{array}$ & $\begin{array}{l}\text { Aditivo } \\
\text { (wt \%) }\end{array}$ \\
\hline \multirow{5}{*}{ 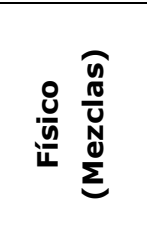 } & rPET & $100: 0$ & - \\
\hline & $75: 25$ & $75: 25$ & - \\
\hline & $50: 50$ & $50: 50$ & - \\
\hline & $25: 75$ & $25: 75$ & - \\
\hline & VPET & $0: 100$ & - \\
\hline \multirow{5}{*}{ 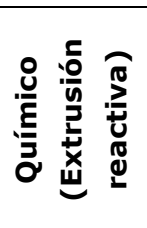 } & rPET_0.25\% & $100: 0$ & 0.25 \\
\hline & $75: 25 \_025 \%$ & $75: 25$ & 0.25 \\
\hline & 50:50_0.25\% & $50: 50$ & 0.25 \\
\hline & $25: 75 \_0.25 \%$ & $25: 75$ & 0.25 \\
\hline & VPET_0.25\% & $0: 100$ & 0.25 \\
\hline
\end{tabular}

Las muestras de las granzas obtenidas a partir de estas metodologías de modificación se muestran en la Figura 4.26. A continuación, se describen los resultados de los distintos ensayos de caracterización realizados. Como se explicó anteriormente, el color amarillo que se observa en la granza del método químico se atribuye a presencia de grupos alquenos formados por la degradación.

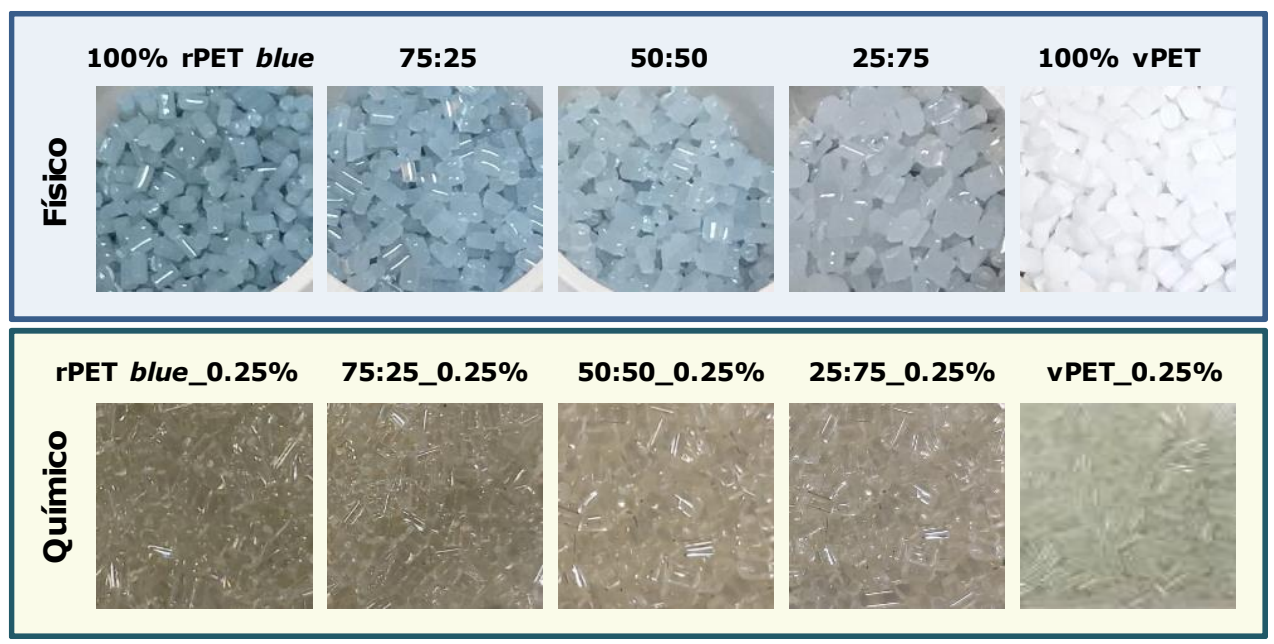

Figura 4.26 Granzas obtenidas en los distintos tratamientos de las mezclas 


\subsubsection{Resultados de la modificación física y modificación química de las mezclas}

\section{Análisis reológico}

En primer lugar, se estudió cómo afectaban estas modificaciones sobre la viscosidad en fundido del rPET y sus mezclas mediante reometría dinámica. En la Figura 4.27 se muestran los valores de la viscosidad compleja $\left(n^{*}\right)$ en función la temperatura. Las curvas fueron obtenidas para las mezclas modificadas por el método físico (a) y químico (b). En estas curvas se establecieron los límites de viscosidad que son válidos para los procesos de obtención de los composites finales (entre $25 \mathrm{~Pa} \cdot \mathrm{s}$ y $100 \mathrm{~Pa} \cdot \mathrm{s})^{24}$. La flecha discontinua vertical, indica la temperatura a la que se van a fabricar los perfiles por pultrusión $\left(280^{\circ} \mathrm{C}\right)$.
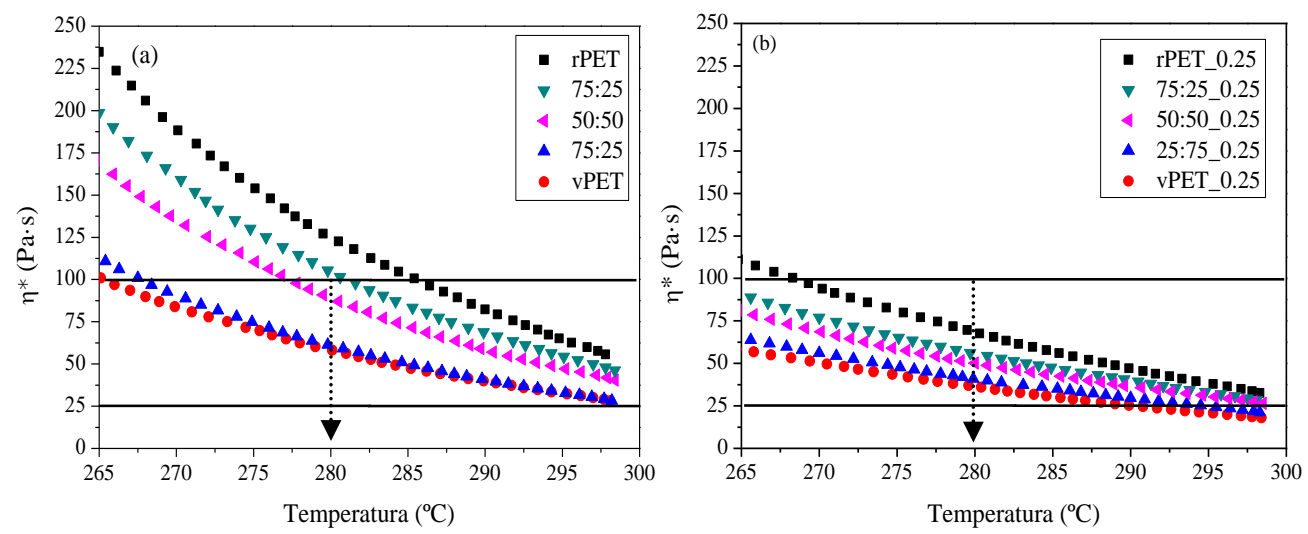

Figura 4.27 Viscosidad compleja de las mezclas de la modificación física (a) y modificación química (b)

En la Figura 4.27 se puede comprobar que al incorporar el VPET la viscosidad disminuye, teniendo más efecto cuanto mayor es la cantidad de VPET en la mezcla. Por otro lado, si se observan los límites de viscosidad recomendados para poder introducir estos materiales en el proceso de impregnación de fibras, se puede ver como estos materiales solamente podrían ser utilizados a altas temperaturas, incluso muy cerca de su degradación.

En la Figura 4.27 (b) se puede ver cómo las mezclas y los polímeros puros modificados químicamente muestran valores de viscosidad más bajos en comparación con las muestras modificadas físicamente (Figura 4.27 (a)). Además, todos los materiales se encuentran dentro del rango de viscosidad delimitado para el proceso de impregnación por lo que, es importante destacar que, este método permitiría utilizar un mayor contenido de material reciclado 
(incluso el $100 \%$ de rPET) para la fabricación de composites termoplásticos por pultrusión.

Como se explicó anteriormente, la disminución en la viscosidad con la incorporación del aditivo se debe a la escisión de las cadenas macromoleculares. Esto se puede comprobar con los valores obtenidos del peso molecular de las muestras modificadas física y químicamente determinados por GPC, que se muestran en la Figura 4.28. A partir de estos resultados, se puede confirmar que el aditivo tiene el mismo efecto que en el VPET puro, es decir es capaz de provocar escisiones de cadenas de forma importante, más aún en mezclas ricas en rPET, posiblemente por la heterogeneidad de estos materiales reciclados.

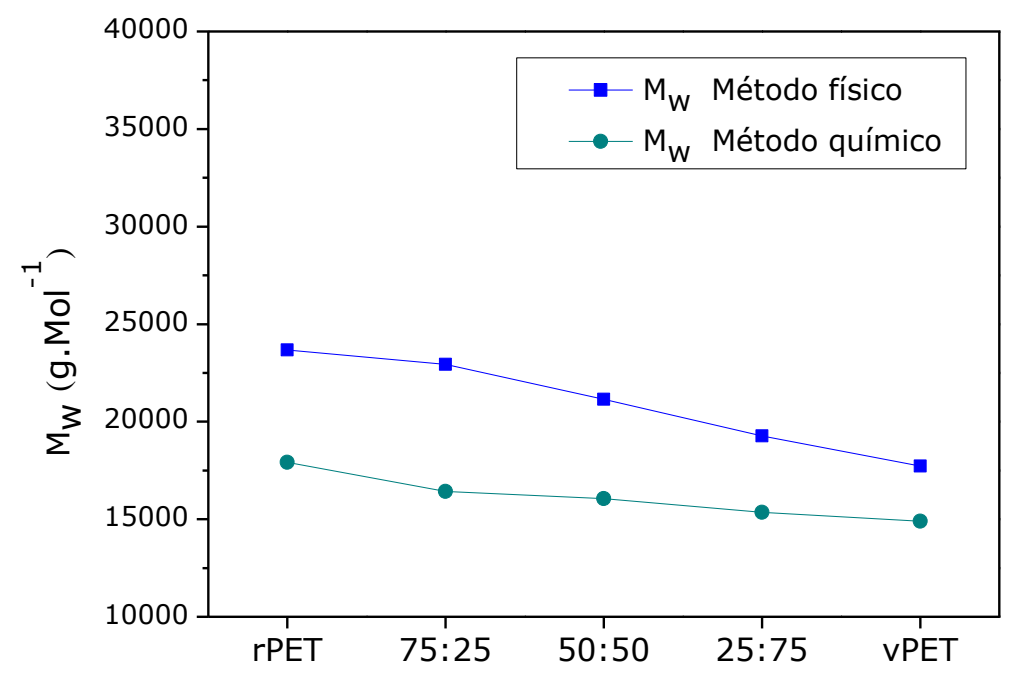

Figura 4.28 Peso molecular de las muestras modificadas física y químicamente 


\section{Propiedades térmicas}

Posteriormente, se realizó el análisis térmico de las muestras obtenidas por el método físico y el químico. En la Figura 4.29 se muestran las curvas de fusión (a) y cristalización (b) del método físico (1) y el método químico (2).

Curvas fusión
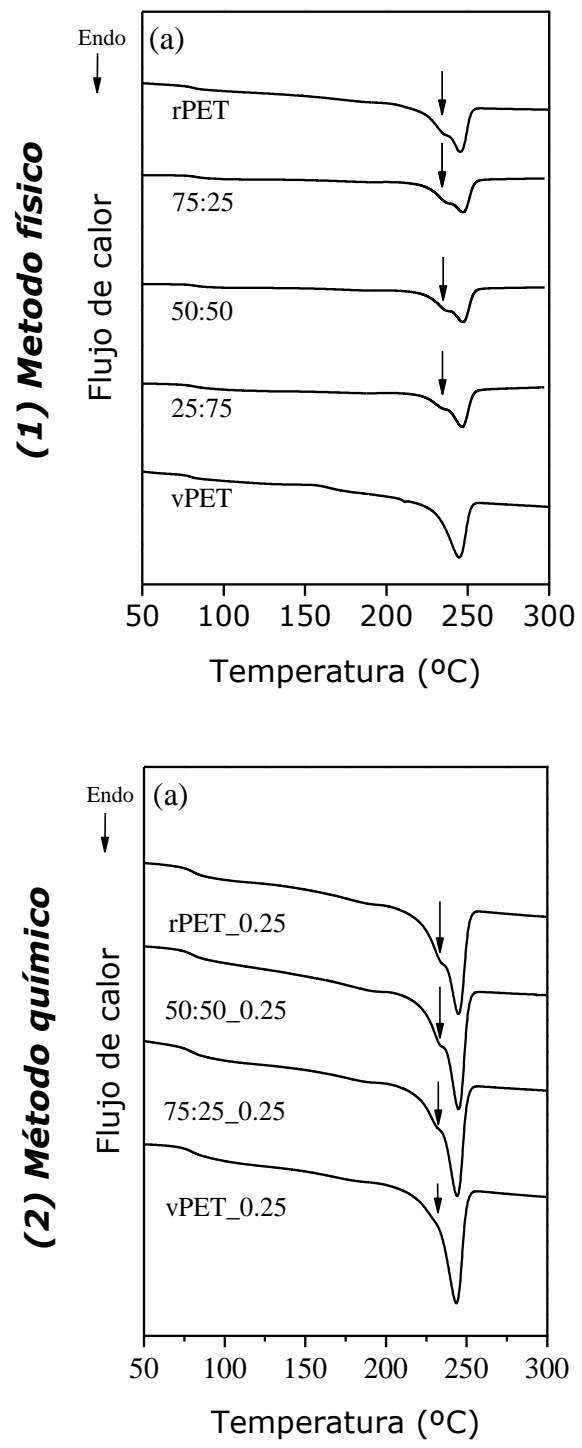

\section{Curvas cristalización}
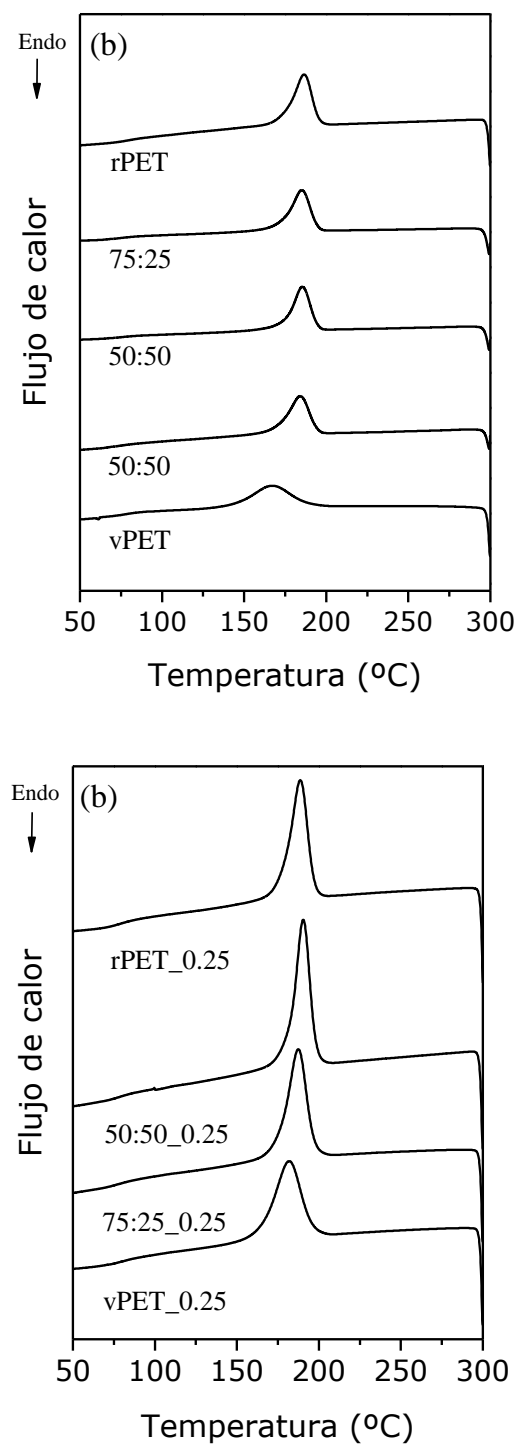

Figura 4.29 Curvas DSC de los polímeros puros y sus mezclas modificados por el método físico (1) y químico (2) 
En la curva de fusión del método físico (Figura 4.29 (1a)), se puede ver que el pico de fusión presenta un hombro a menor temperatura en las mezclas que contienen mayor cantidad de rPET. Estos picos son típicos en los materiales de PET reciclados, debido a la degradación termo-mecánica que han sufrido al ser reprocesados y que, además, han formado distintas secciones cristalinas (de distintos espesores) $)^{19,25}$.

En las curvas de fusión del método químico (Figura 4.29 (2a)) se puede observar, al igual que en el método físico, un segundo pico en la fusión endotérmica; en este caso, se debe a la suma del efecto del aditivo y del contenido de material reciclado. Este pico aparece a menor temperatura que el pico principal de fusión, lo cual indica que al añadir el aditivo se forman cadenas poliméricas más cortas que pueden fundir a menor temperatura. Como se puede ver en ambos métodos, el pico principal de fusión $\left(T_{f}\right)$ se mantiene aproximadamente a la misma temperatura en todas las muestras.

A continuación, en la Tabla 4.16 se presentan los valores de las propiedades térmicas obtenidos de las curvas DSC.

Tabla 4.16 Composición y propiedades térmicas de las muestras modificadas física y químicamente

\begin{tabular}{|c|c|c|c|c|c|}
\hline Muestra & RPET: vPET & $T_{g} \pm 0.5^{\circ} \mathrm{C}$ & $T_{f} \pm 0.5^{\circ} \mathrm{C}$ & $T_{c} \pm 0.5^{\circ} \mathrm{C}$ & $X \pm 1 \%$ \\
\hline \multirow{5}{*}{$\begin{array}{l}\text { Modificación física } \\
\text { (Mezclas) }\end{array}$} & $100: 0$ & 81.1 & 245.3 & 186.5 & 27 \\
\hline & $75: 25$ & 82.0 & 247.5 & 184.8 & 22 \\
\hline & $50: 50$ & 82.8 & 247.4 & 185.1 & 23 \\
\hline & $25: 75$ & 81.7 & 246.8 & 183.7 & 24 \\
\hline & $0: 100$ & 80.1 & 244.6 & 166.7 & 27 \\
\hline \multirow{5}{*}{$\begin{array}{l}\text { Modificación química } \\
\text { (extrusión reactiva de } \\
\text { las mezclas) }\end{array}$} & rPET_0.25 & 80.3 & 244.9 & 188.2 & 26 \\
\hline & 75:25_0.25 & 79.9 & 244.9 & 190.5 & 27 \\
\hline & 50:50_0.25 & 79.8 & 244.9 & 190.4 & 28 \\
\hline & 25:75_0.25 & 80.7 & 244.1 & 187.4 & 28 \\
\hline & VPET_0.25 & 79.9 & 243.6 & 181.6 & 27 \\
\hline
\end{tabular}

Estos datos se han representado en la Figura 4.30, donde se puede ver que las mezclas modificadas por la vía química mostraron valores de temperatura de fusión más bajos que las mezclas modificadas físicamente. Este hecho corroboró que estructuras cristalinas más pequeñas, necesitan menos energía para fundir; con lo cual se vuelve a demostrar el potencial que tiene el método químico para la reducción de $\mathrm{Mw}$. 


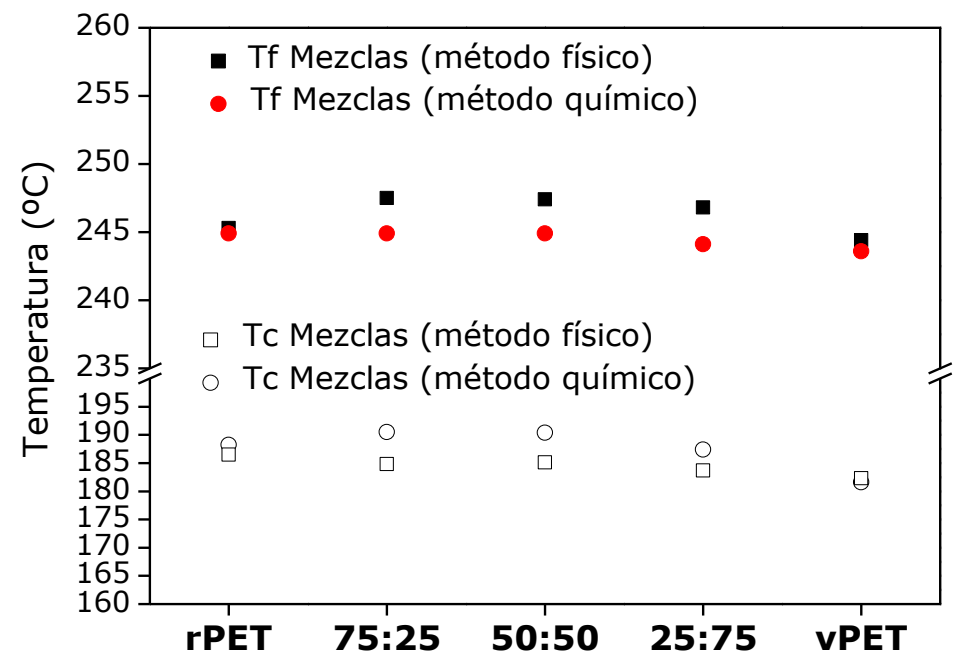

Figura 4.30 Valores de $T_{f}$ y $T_{c}$ de la modificación física y química

En cuanto a la cristalización, es de esperar que, como se demostró en el estudio de las propiedades térmicas de las otras modificaciones propuestas, las cadenas más cortas con temperaturas de fusión más pequeñas, tengan mayor facilidad para cristalizar y sean quienes alcancen la máxima cristalización (altos porcentajes) a temperaturas más altas (Figura 4.31); por este motivo, las muestras sometidas a modificación química presentan este comportamiento.

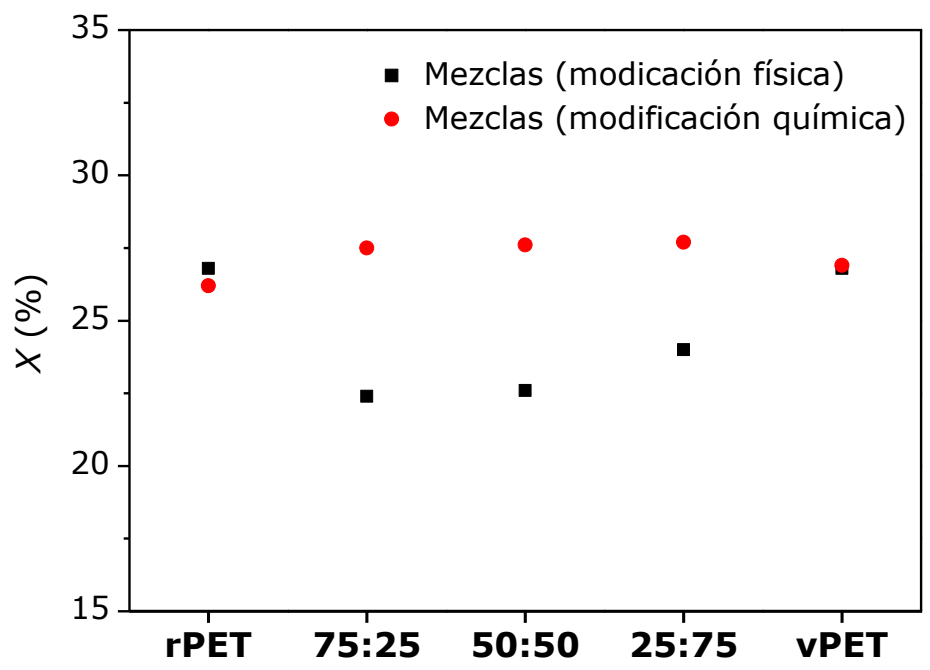

Figura 4.31 Valores de cristalinidad de la modificación física y química 


\section{Propiedades mecánicas}

Por último, se evaluaron las propiedades mecánicas de las muestras modificadas física y químicamente. Los valores de Módulo de Young de las muestras obtenidas por ambos métodos se presentan en la Figura 4.32. Estos resultados son de suma importancia ya que son los que demostrarán sí las pérdidas de $\mathrm{Mw}$ traen consigo pérdidas importantes de propiedades mecánicas o sí por el contrario la definición de mezclas, entre matrices de distinta viscosidad y la incorporación del aditivo, logra mantener las propiedades mecánicas.

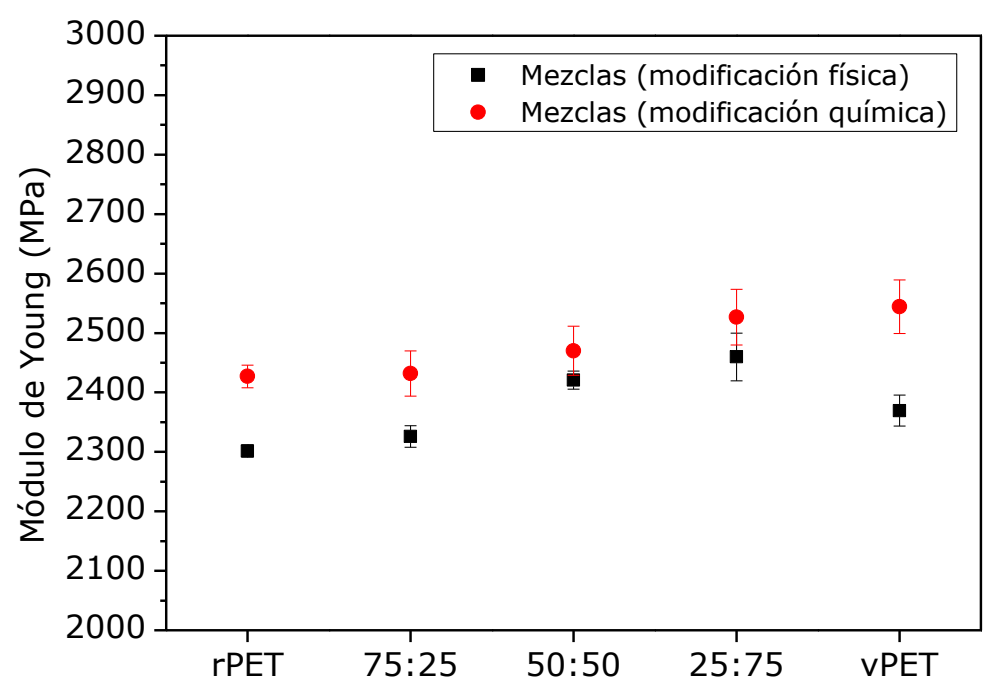

Figura 4.32 Módulo de Young de mezclas modificadas física (ם) y químicamente (•)

En la Figura 4.32 se puede ver que la adición de vPET en mezclas (a través del método físico) produce un ligero aumento en el Módulo de Young respecto la muestra de rPET. Por lo tanto, se puede decir que, a la vez que se consigue disminuir la viscosidad al incorporar el VPET, el método físico también proporciona una manera de mejorar el módulo elástico del material reciclado. En la Figura también se puede ver que con la incorporación del aditivo en las matrices puras y en las mezclas a través del método químico se consiguen valores más altos de Módulo de Young que el método físico. Este hecho puede deberse a la inserción de los grupos aromáticos que constituyen la estructura molecular del aditivo, que actúan como rigidizadores de la estructura polimérica, a los efectos de tener mayor cantidad de cristales de menor tamaño, por las escisiones de cadena que se han producido y por los efectos de la co-cristalización que, posiblemente tenga una fase de PET sobre la otra; por ej. las fracciones de rPET, de alta temperatura de fusión, pueden nuclear las secciones de bajo peso molecular del vPET. 
Finalmente, aun cuando se ha logrado mantener la rigidez, es muy importante evaluar la tenacidad. En este sentido, la representación de los valores de impacto que se presenta en la Figura 4.33, muestra que las mezclas sin tratamiento químico mantienen e incluso mejoran esta propiedad con la adición del VPET. Por otra parte, como era esperado, las escisiones provocadas por el injerto químico pueden llegar a disminuir la energía de absorción durante el impacto, sin embargo, no hay pérdidas tan importantes (por debajo de los $1 \mathrm{KJ} / \mathrm{m}^{2}$ ), como se manifestaba en las modificaciones hechas sólo con el aditivo o las inducidas por los parámetros propios del procesado: termo-cizalla o el efecto de la hidrólisis. Esto sin duda, demuestra que las mezclas representan una herramienta útil para modelar las propiedades reológicas, sin excesivas pérdidas en las propiedades mecánicas.

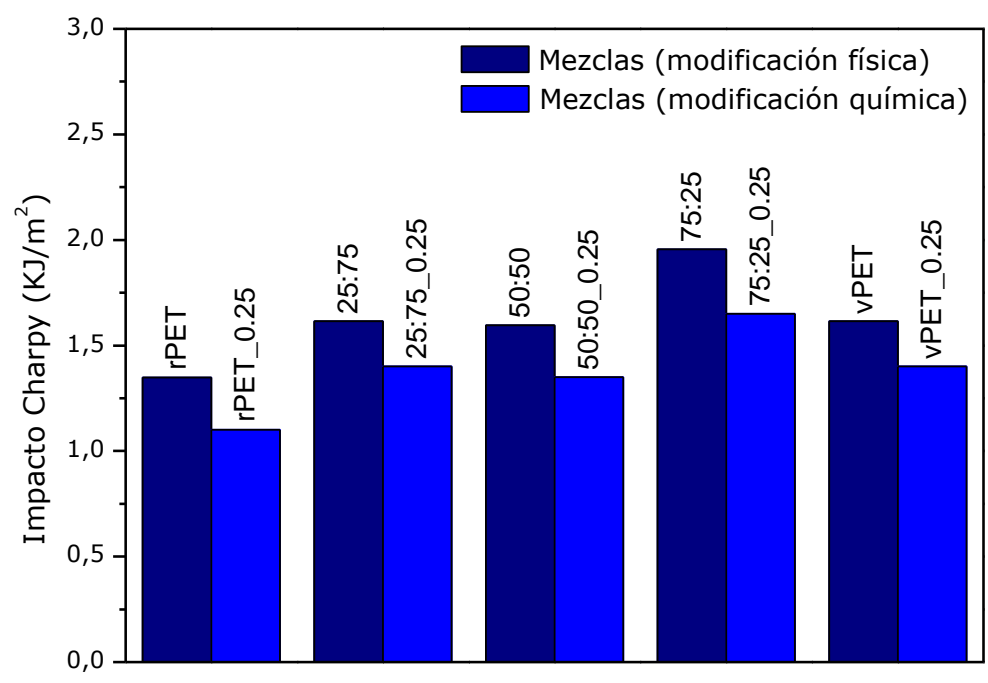

Figura 4.33 Impacto Charpy de mezclas modificadas física y químicamente

En la Figura 4.34 se representan los valores de resistencia a tracción de las muestras modificadas física y químicamente. Como se puede apreciar, la incorporación del aditivo también provocó cambios en la resistencia a tracción de los materiales. Las muestras modificadas mediante el método químico mostraron valores más bajos de resistencia a la tracción que las muestras modificadas físicamente. Este resultado está relacionado con los obtenidos anteriormente de las propiedades térmicas, donde los cristales formados a través del método físico son más grandes, los cuales soportan más carga que los cristales formados por el método químico (cristales más pequeños). 


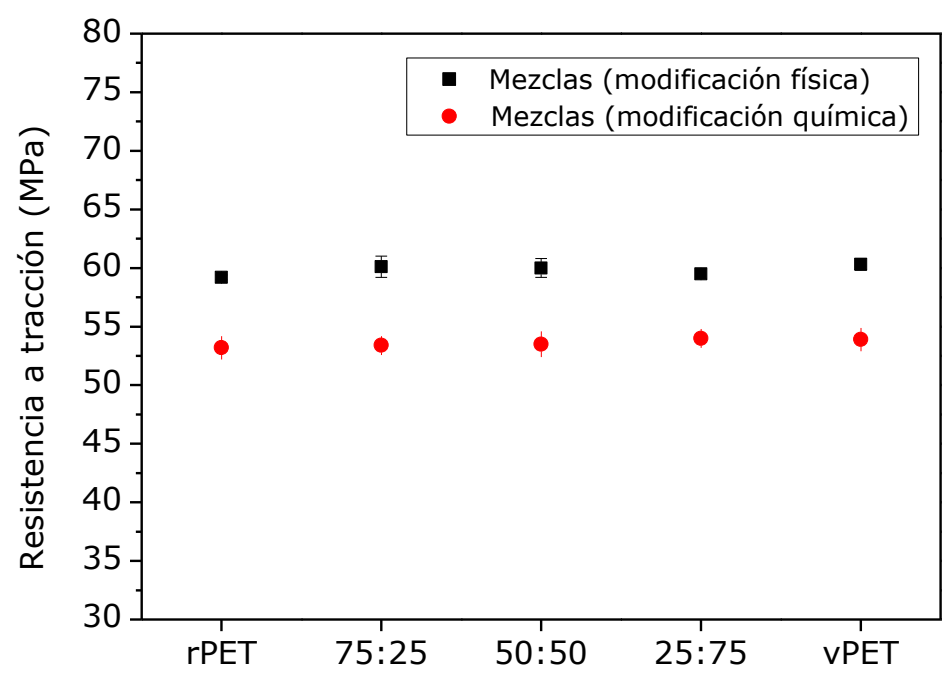

Figura 4.34 Resistencia a la tracción de mezclas modificadas física ( $\square$ ) y químicamente $(\bullet)$

\subsection{CONCLUSIONES}

En este capítulo se ha estudiado, en primer lugar, la degradación térmica, hidrolítica y química sobre el VPET. De esta parte de la investigación se seleccionaron y estudiaron las condiciones de extrusión, el efecto del agua y la cantidad de aditivo que se utilizarían en la segunda parte de la investigación.

Posteriormente, se evaluaron dos métodos (físico y químico) para disminuir la viscosidad del rPET y al mismo tiempo mantener las propiedades mecánicas. De este trabajo, se han extraído las siguientes conclusiones:

- La modificación reológica mediante el método físico a través de las mezclas (rPET/vPET) ha permitido disminuir los valores de viscosidad del rPET con la incorporación del VPET. Además, esta incorporación ha dado lugar a un aumento en el Módulo de Young.

- La modificación reológica mediante la vía química ha permitido obtener valores de viscosidad inferiores a los del método físico. El injerto químico del ácido 5 amino isoftálico en las matrices de PET (virgen y reciclado) y sus mezclas, ha tenido un importante efecto en la estructura de los poliésteres. Se han creado mayor porcentaje de poblaciones cristalinas, pero de menor tamaño (caída de $\mathrm{Mw}$ ); por este motivo ha existido una relación de compromiso entre las propiedades mecánicas y la viscosidad. La reacción es extremadamente sensible a la cantidad del modificante y a las condiciones de procesado. 
Por lo tanto, ambos métodos permitirían procesar rPET con baja viscosidad, permitiendo el uso de presiones y/o temperaturas más bajas para su aplicación en la fabricación de composites por pultrusión. El método químico permitiría utilizar el $100 \%$ de rPET y el método físico un máximo del $50 \%$ de rPET como matriz para la fabricación de composites por pultrusión.

A partir de estas conclusiones se seleccionaron los materiales más válidos, en función de su viscosidad y morfología, para la obtención de los perfiles mediante los procesos de pultrusión (Capítulo 5).

\subsection{REFERENCIAS BIBLIOGRÁFICAS}

1. Sammon C, Yarwood J, Everall N. FT-IR study of the effect of hydrolytic degradation on the structure of thin PET films. Polym Degrad Stab. 2000;67(1):149-158. doi:10.1016/S0141-3910(99)00104-4

2. Masmoudi F, Fenouillot F, Mehri A, Jaziri M, Ammar E. Characterization and quality assessment of recycled post-consumption poly(ethylene terephthalate) (PET). 2018;(June). doi:10.1007/s11356-018-2390-7

3. Tuffi R, D'Abramo S, Cafiero LM, Trinca E, Vecchio Ciprioti S. Thermal behavior and pyrolytic degradation kinetics of polymeric mixtures from waste packaging plastics. Express Polym Lett. 2018;12(1):82-99. doi:10.3144/expresspolymlett.2018.7

4. Oromiehie A, Mamizadeh A. Recycling PET beverage bottles and improving properties. Polym Int. 2004;53(6):728-732. doi:10.1002/pi.1389

5. Assadi $R$, Colin $X$, Verdu J. Irreversible structural changes during PET recycling by extrusion. Polymer (Guildf). 2004;45(13):4403-4412. doi:10.1016/j.polymer.2004.04.029

6. Nait-Ali LK, Colin X, Bergeret A. Kinetic analysis and modelling of PET macromolecular changes during its mechanical recycling by extrusion. Polym Degrad Stab. 2011;96(2):236-246. doi:10.1016/j.polymdegradstab.2010.11.004

7. Silva Spinacé MA, De Paoli MA. Characterization of poly(ethylene terephthalate) after multiple processing cycles. J Appl Polym Sci. 2001;80(1):20-25. doi:10.1002/1097-4628(20010404)80:1<20::AID-APP1069>3.0.CO;2-S

8. Awaja F, Pavel D. Recycling of PET. Eur Polym J. 2005;41(7):1453-1477. doi:10.1016/j.eurpolymj.2005.02.005

9. Samperi F, Puglisi C, Alicata R, Montaudo G. Thermal degradation of poly(ethylene terephthalate) at the processing temperature. Polym Degrad Stab. 2004;83(1):310. doi:10.1016/S0141-3910(03)00166-6

10. Bertolottp B, Rospigliosp C, Nakamatsu J. Poliésteres y Reciclaje Químico del Poli ( tereftalato de etileno ). 2005;(7):13-20.

11. Borrás APR. ESTUDIO DE LAS CONDICIONES ÓPTIMAS DE TRANSFORMACIÓN PARA EL RECICLADO MECÁNICO DEL POLIETILENTEREFTALATO (PET). 2006;18:314.

12. Park R, Sridhar V, Park H. Taguchi method for optimization of reaction conditions in microwave glycolysis of waste PET. J Mater Cycles Waste Manag. 2019;(0123456789). doi:10.1007/s10163-019-00958-7

13. Sharma GVSS, Rao RU, Rao PS. A Taguchi approach on optimal process control parameters for HDPE pipe extrusion process. J Ind Eng Int. 2017;13(2):215-228. doi:10.1007/s40092-016-0179-1

14. Del Mar Castro López M, Ares Pernas AI, Abad López MJ, Latorre AL, López Vilariño JM, González Rodríguez MV. Assessing changes on poly(ethylene terephthalate) properties after recycling: Mechanical recycling in laboratory versus postconsumer recycled material. Mater Chem Phys. 2014;147(3):884-894. doi:10.1016/j.matchemphys.2014.06.034

15. Härth M, Kaschta J, Schubert DW. Rheological study of the reaction kinetics in a 
poly(ethylene terephthalate) melt. Polym Degrad Stab. 2015;120:70-75. doi:10.1016/j.polymdegradstab.2015.06.001

16. Frounchi M. Studies on degradation of PET in mechanical recycling. Macromol Symp. 1999;144:465-469. doi:10.1002/masy.19991440142

17. Sanches NB, Dias ML, Pacheco EBAV. Comparative techniques for molecular weight evaluation of poly (ethylene terephthalate) (PET). Polym Test. 2005;24(6):688-693. doi:10.1016/j.polymertesting.2005.05.006

18. Farah S, Kunduru KR, Basu A, Domb AJ. Molecular Weight Determination of Polyethylene Terephthalate. Elsevier Inc.; 2015. doi:10.1016/B978-0-323-313063.00008-7

19. Badía JD, Vilaplana F, Karlsson S, Ribes-Greus A. Thermal analysis as a quality tool for assessing the influence of thermo-mechanical degradation on recycled poly(ethylene terephthalate). Polym Test. 2009;28(2):169-175. doi:10.1016/j.polymertesting.2008.11.010

20. Van Kets K, Delva L, Ragaert K. Structural stabilizing effect of SEBSgMAH on a PPPET blend for multiple mechanical recycling. Polym Degrad Stab. 2019;166:60-72. doi:10.1016/j.polymdegradstab.2019.05.012

21. Xanthos M. Reactive Extrusion: Principles and Practice. Munich; 1992.

22. Ramírez-Hernández A, Valera-Zaragoza M, Aparicio-Saguilán A, Conde-Acevedo JC. Thermal behavior of banana starch films with degraded polyethylene terephthalate. Rev Mex Ing Quim. 2015;14(2):513-521.

23. Besnoin JM, Choi KY. Identification and Characterization of Reaction Byproducts in the Polymerization of Polyethylene Terephthalate. J Macromol Sci Part C. 1989;29(1):55-81. doi:10.1080/07366578908055164

24. Kazemi Najafi S. Use of recycled plastics in wood plastic composites - A review. Waste Manag. 2013;33(9):1898-1905. doi:10.1016/j.wasman.2013.05.017

25. Awaja F, Pavel D. Recycling of PET. Eur Polym J. 2005;41(7):1453-1477. doi:10.1016/j.eurpolymj.2005.02.005 


\section{Ca pítu I o 5}

En este capítulo se muestra la segunda fase de este trabajo de investigación, centrada en la introducción de PET reciclado como matriz polimérica en los procesos de pultrusión. En primer lugar, se llevó a cabo el estudio de la impregnación de las matrices obtenidas en el capítulo anterior por el método físico y químico en la cámara diseñada para este fin. A continuación, se muestra la obtención de perfiles a partir de las dos tecnologías de pultrusión (en fundido y en polvo), así como la caracterización completa de los perfiles obtenidos. Finalmente, se comparan con las propiedades de los perfiles obtenidos con las de perfiles termoestables tradicionalmente usados.

\section{Obtención y caracterización de perfiles}

\subsection{PRUEBAS DE IMPREGNACIÓN}

\subsubsection{Materiales}

El grado de impregnación de las matrices desarrolladas en el capítulo anterior por el método físico y químico (Figura 5.1) fue evaluado en la cámara de impregnación diseñada a partir de la obtención de pre-impregnados (tapes).

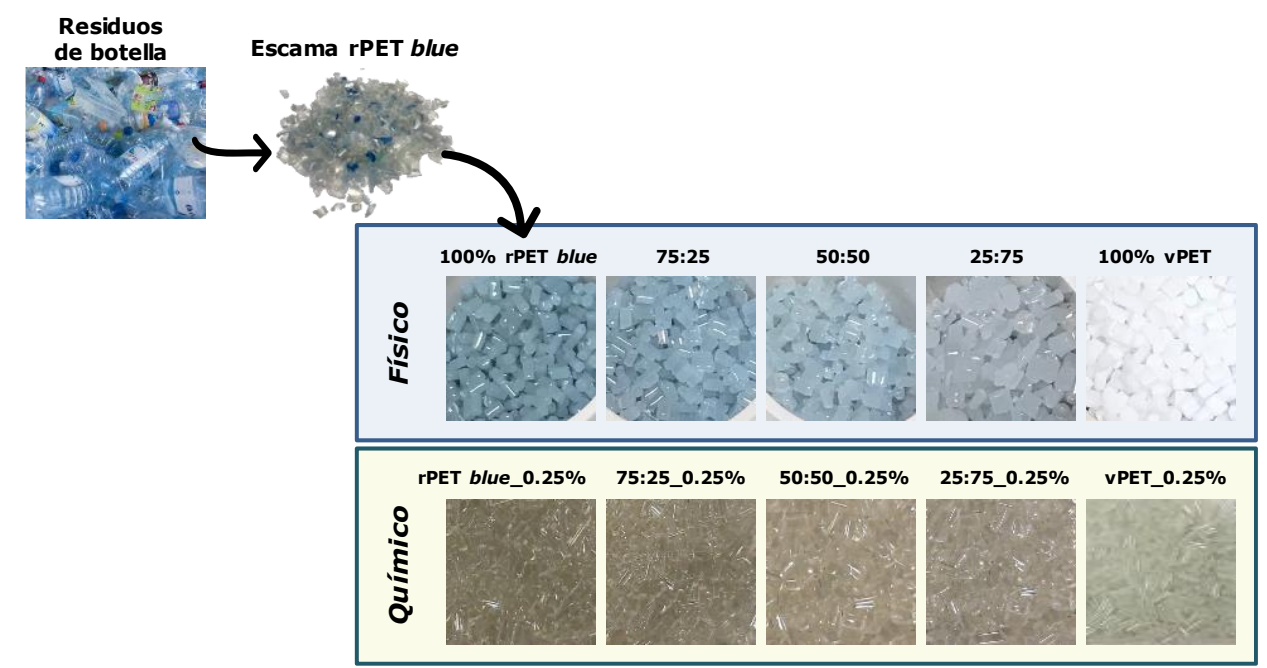

Figura 5.1 Matrices modificadas reológicamente por el método físico y químico

La fibra de vidrio que se utilizó para la obtención de los tapes fue de tipo 305ETYPE 30 suministrada por Owens Corning, cuyas propiedades técnicas se muestran en la Tabla 5.1. 
Esta fibra fue utilizada tanto para el estudio de la impregnación de las matrices, como para la obtención de los perfiles por los dos métodos de pultrusión (en granza y en polvo).

Tabla 5.1 Propiedades de la fibra de vidrio

\begin{tabular}{ccc}
\hline & Propiedad & Fibra de vidrio \\
\cline { 2 - 3 } & Densidad lineal $(\mathrm{Tex})$ & 2400 \\
Densidad $\left(\mathrm{kg} / \mathrm{m}^{3}\right)$ & 2650 \\
& Resistencia a la tracción $(\mathrm{MPa})$ & 3500 \\
& Módulo de Young $(\mathrm{GPa})$ & 17 \\
& Diámetro de fibra $(\mu \mathrm{m})$ & 17 \\
\hline
\end{tabular}

\subsubsection{Obtención de tapes (pre-impregnado)}

Las pruebas de impregnación se llevaron a cabo en la cámara construida para este estudio (descrita en el Capítulo 3). En la Figura 5.2 se muestra la imagen de la cámara acoplada a la línea de extrusión. Para la elaboración de los tapes, se introduce la matriz en forma de granza en la extrusora a una temperatura de $265^{\circ} \mathrm{C}$ y una velocidad de husillo de 100rpm. Una vez que la matriz fundida recorre todo el barril de la extrusora, se encuentra con la fibra de vidrio en la cámara diseñada donde se produce la impregnación. La temperatura de la cámara de impregnación fue fijada a $285^{\circ} \mathrm{C}$.

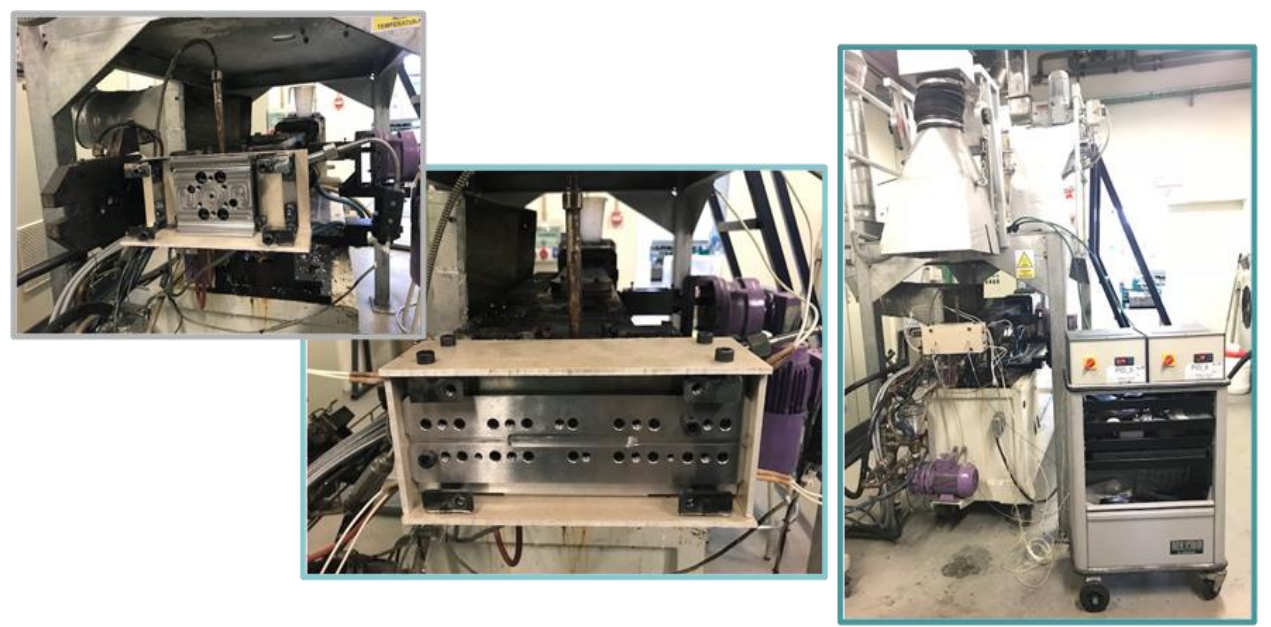

Figura 5.2 Cámara de impregnación acoplada a la línea de extrusión 
Como se explicó anteriormente, uno de los factores que afectan a la impregnación es la viscosidad de las matrices. Los polímeros termoplásticos disponibles en el mercado tienen una alta viscosidad en estado fundido, típicamente superior a 200Pa.s (uno o dos órdenes superior a los termoestables) ${ }^{1}$, lo que hace que la impregnación de la fibra sea difícil, sobre todo, cuando la proporción del contenido de fibra es alta, en particular cuando es mayor del $50 \%$ en volumen.

La utilización de este tipo de polímeros de alta viscosidad requiere tiempos de impregnación prolongados, es decir, una velocidad de tiro (velocidad con la que se tracciona de la fibra) muy lenta ${ }^{2}$. En la mayoría de los casos, los perfiles obtenidos de estas matrices pueden tener micro cavidades y zonas mal impregnadas, perjudiciales para sus propiedades mecánicas ${ }^{3}$. Este fenómeno de pérdida de propiedades se ve más perjudicado cuando aumenta la velocidad de tiro.

En este trabajo, se estudió la influencia de la velocidad de tiro en la impregnación, durante la obtención de los tapes a dos velocidades: $0.2 \mathrm{~m} / \mathrm{min}$ y a una velocidad más alta $0.5 \mathrm{~m} / \mathrm{min}$. Estas velocidades fueron ajustadas durante el proceso de obtención de los tapes, como la mínima y máxima posible. En la Figura 5.3 se muestra una imagen de los tapes obtenidos.

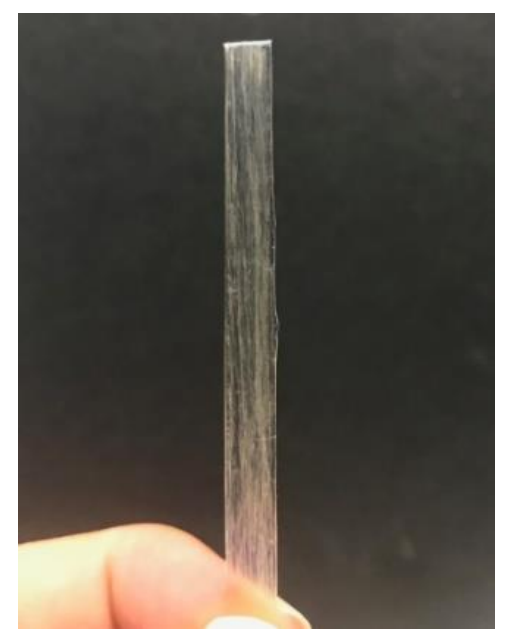

Figura 5.3 Imagen de tape obtenido en la cámara de impregnación 


\subsubsection{Caracterización de los tapes}

La caracterización de los tapes se llevó a cabo por microscopía electrónica de barrido (SEM), a partir de la cual se pudo evaluar la impregnación y adhesión de cada matriz con la fibra. El grado de impregnación también se valora a partir de la cantidad de matriz que contienen los tapes obtenido por calcinación.

En primer lugar, se muestran los resultados de impregnación de los polímeros puros (VPET y rPET) y éstos modificados químicamente (vPET_0.25\% y rPET_0.25\%). A continuación, se muestra la impregnación de las mezclas (rPET:VPET) obtenidas a partir del método físico y modificadas químicamente.

En la Figura 5.4 se muestran las micrografías de los tapes de PET virgen (VPET) y el PET virgen modificado químicamente (VPET_0.25\%) obtenidos a diferentes velocidades de tiro. Todos los tapes fueron analizados en su sección transversal como se muestra en la imagen.
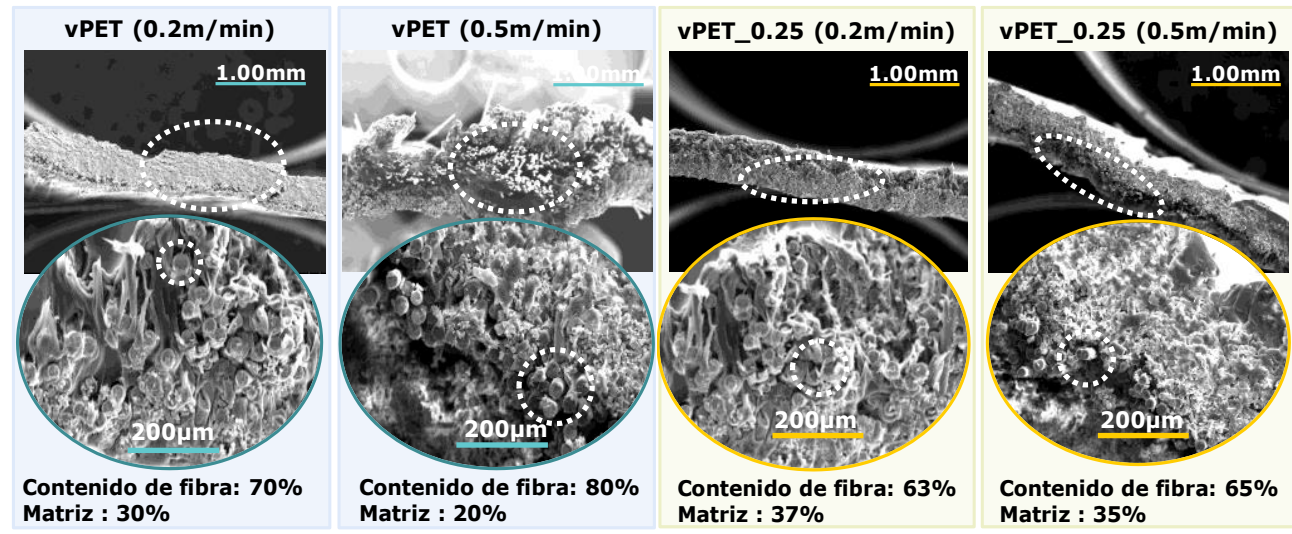

Figura 5.4 Análisis SEM de los tapes de VPET y VPET_0.25\% obtenidos en la cámara de impregnación

Como se puede apreciar, cuando comparamos los tapes obtenidos a una velocidad de tiro más baja $(0.2 \mathrm{~m} / \mathrm{min})$ estos presentan un grado mayor de impregnación, mostrando matriz en toda la sección y la fibra completamente recubierta por la matriz. Cuando se eleva la velocidad de tiro $(0.5 \mathrm{~m} / \mathrm{min})$, la fibra y la matriz tienen menos tiempo de permanencia en la cámara, con lo cual el grado de impregnación es menor y aparecen algunos vacíos y menor cantidad de matriz alrededor de las fibras.

Si se evalúa la micrografía de vPET_0.25\% se puede apreciar cómo, a partir de esta matriz modificada reológicamente, se consigue una excelente impregnación, incluso a altas velocidades de tiro (alto contenido de matriz en el tape (35\%)). Como vimos en los resultados del capítulo anterior el vPET_0.25\% presentó valores 
más bajos de viscosidad que el VPET, por lo que, a partir de estos resultados, se puede determinar que la disminución de la viscosidad a través del método químico mejora la impregnación de los tapes.

Este hecho indica que la velocidad de tiro es un parámetro de procesado importante de controlar que influye en la calidad y cantidad de la impregnación. En este caso, el uso de estas matrices de alta fluidez ha permitido obtener tapes preimpregnados con un contenido de matriz adecuado incluso a la velocidad más alta fijada.

Posteriormente, se analizó la impregnación de la matriz de PET reciclado ( $\mathrm{FPET}$ ). En la Figura 5.5 se muestran las micrografías SEM de los tapes con rPET sin modificar (rPET) y el rPET modificado químicamente (rPET_0.25\%) obtenidos a diferentes velocidades de tiro.
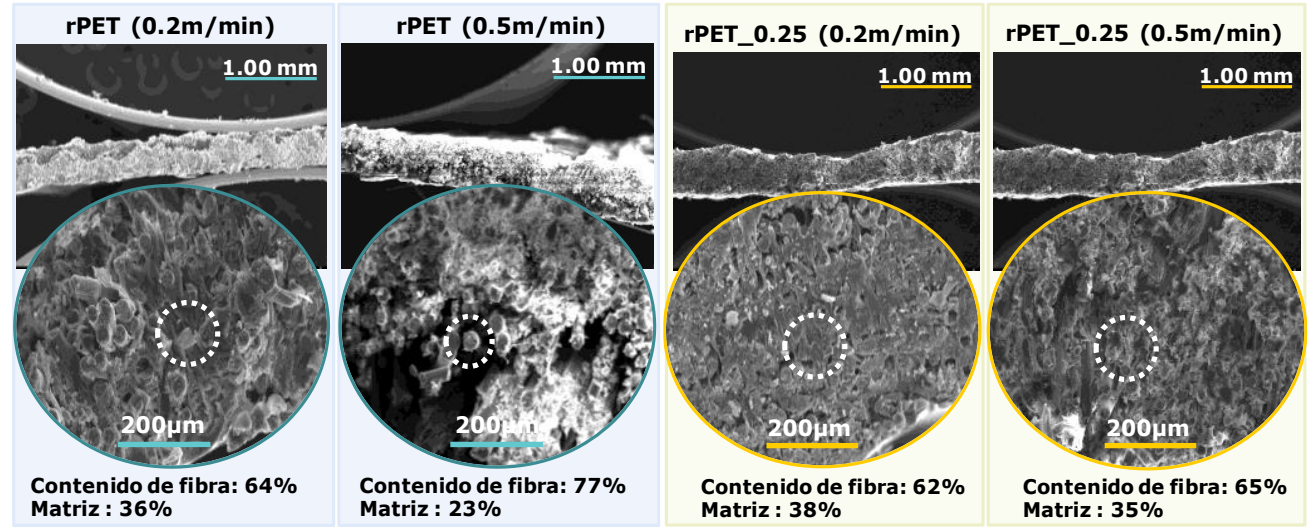

Figura 5.5 Análisis SEM de los tapes de rPET y rPET_0.25\% obtenidos en la cámara de impregnación

Al igual que ocurre en los tapes obtenidos con vPET, en la Figura se puede observar como el tape de rPET que se obtuvo a una menor velocidad de tiro $(0.2 \mathrm{~m} / \mathrm{min})$ presentó mayor grado de impregnación $(36 \%)$, donde se puede apreciar las fibras totalmente recubiertas por la matriz, sin presencia de vacíos entre la fibra y la matriz. Cuando se eleva la velocidad de tiro $(0.5 \mathrm{~m} / \mathrm{min})$ se observa que el tape presenta algunos vacíos (señalados con círculos discontinuos), que como se explicó anteriormente, pueden repercutir en las propiedades mecánicas finales del perfil. Sin embargo, cuando el rPET tiene menos viscosidad (rPET_0.25\%) se puede ver cómo se mejora la impregnación de la matriz con la fibra (aumenta la cantidad de matriz en el tape), obteniendo buenos resultados incluso a velocidad de tiro más alta (35\%). 
Estos resultados permiten concluir que estas matrices de alta fluidez permiten alcanzar un grado de impregnación de las fibras adecuado, incluso a una velocidad alta. A continuación, en la Figura 5.6 se muestran las micrografías de los tapes obtenidos a partir de las mezclas de polímeros (rPET:vPET) física y químicamente modificadas.
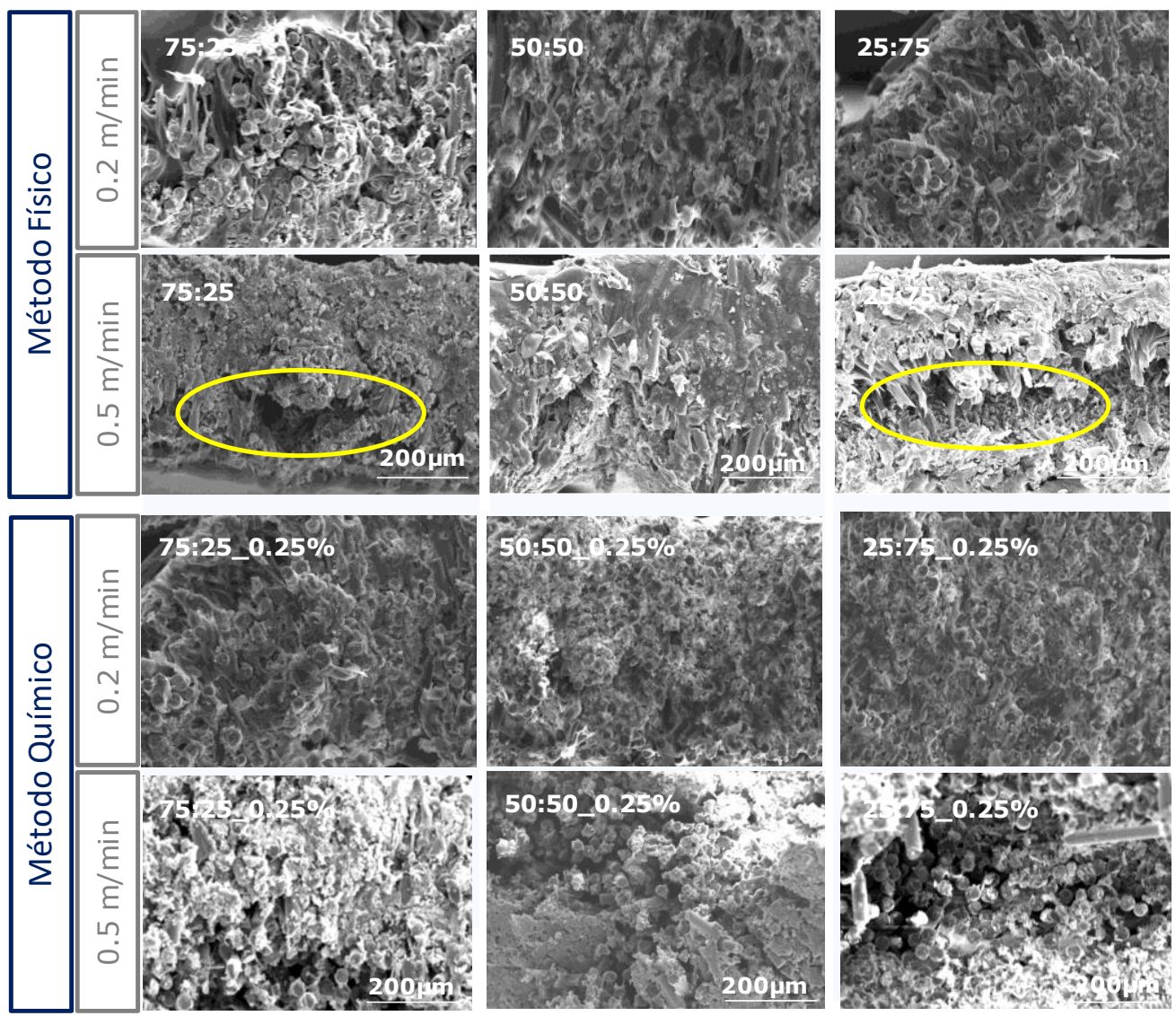

Figura 5.6 Análisis SEM de los tapes obtenidos con matrices de la modificación física y química

Como ya se ha observado anteriormente en los polímeros puros, cuando se aumenta la velocidad de tiro se producen tapes con menor grado de impregnación. En la Figura 5.6 se puede ver como a velocidad de tiro más alta aparecen vacíos que forman grietas en el pre-impregnado (círculos); con la diferencia que, a partir del método químico se observaron menos cantidad de vacíos o partes de fibras sin impregnar por la matriz, esto se corrobora con los datos de calcinación recogidos en la Figura 5.7. 
En la Figura 5.7 se muestra el volumen de matriz de los tapes obtenidos a partir de las muestras modificadas física y químicamente. Como se puede apreciar, los tapes que contienen mayor volumen de matriz son lo que se produjeron con los materiales modificados químicamente, lo cual nos indica que el método de modificación química mejora la adhesión de la matriz a las fibras.

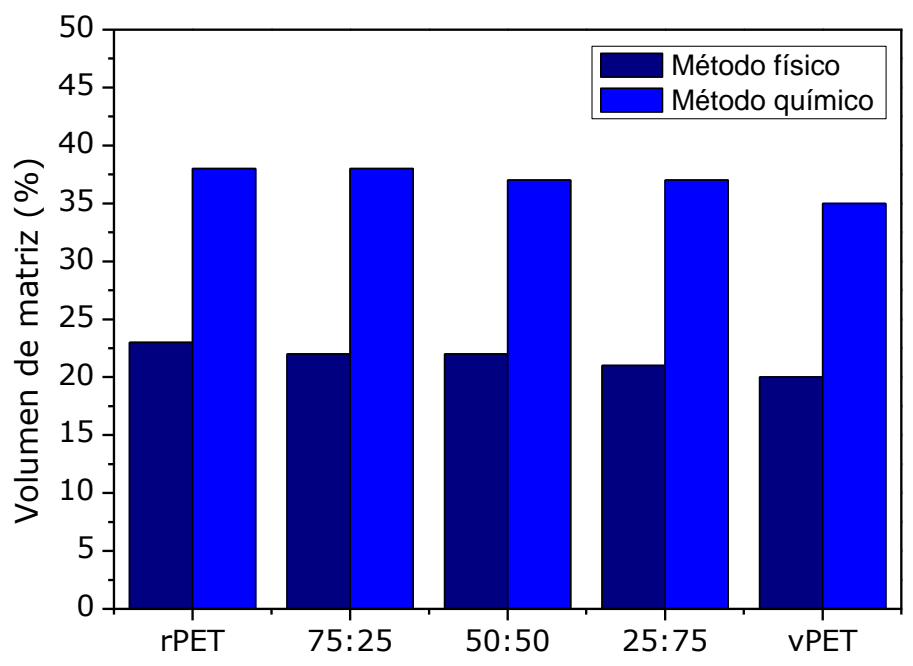

Figura 5.7 Volumen de matriz de los tapes obtenidos con las matrices física y químicamente modificadas

Una vez se comprobó la impregnación de las matrices, se seleccionó el material más conveniente para la obtención de un producto final, es decir, los perfiles a partir de los métodos de pultrusión explicados (en fundido y en polvo).

Debido a que a partir del rPET se obtuvo una buena impregnación, incluso a una velocidad de tiro más alta, se decidió trabajar con esta matriz en los procesos de pultrusión, atendiendo también a que al ser una matriz residual aporta beneficios de sostenibilidad al perfil fabricado.

Además del rPET, se seleccionó su análogo modificado químicamente, rPET_0.25\%, ya que, este tratamiento, fue quién mostró mayor volumen de matriz (mayor grado de impregnación) en los tapes de rPET, independientemente de la composición de la mezcla. De esta manera se puede estudiar la influencia de la viscosidad en las propiedades del perfil o la pieza final. Estos dos materiales fueron utilizados como matrices en ambos procesos de pultrusión, en fundido y en polvo. 


\subsection{PULTRUSIÓN EN FUNDIDO}

\subsubsection{Obtención de perfiles}

Como se explicó anteriormente, en esta tecnología de pultrusión la matriz polimérica es utilizada en forma de granza directamente, tras haber sido extruida. Por ello, en primer lugar, se preparó por extrusión un material con un contenido del $100 \%$ de material reciclado (rPET blue), y el mismo modificado por extrusión reactiva (rPET_0.25\%). Las condiciones de extrusión utilizadas fueron $265^{\circ} \mathrm{C}$ como temperatura de proceso, 100rpm de velocidad de husillo y $5 \mathrm{Kg} / \mathrm{h}$ de caudal (seleccionadas a partir del DOE del Capítulo anterior). Para la producción de perfiles fue necesario preparar $50 \mathrm{~kg}$ de cada material por extrusión.

Posteriormente, los materiales en forma de granza preparados por extrusión, fueron enviados a la empresa CirComp donde se llevó a cabo la obtención de los perfiles en un proceso industrializado de pultrusión en fundido. La fibra de vidrio que se utilizó se detalla en la Tabla 5.1 en la sección de materiales. El rPET_0.25\% fue descartado para la consolidación por razones de procesabilidad, a pesar de estar en los límites de viscosidad requeridos por el proceso; reprocesar el rPET_0.25\% provoca una caída aún mayor de la viscosidad, produciéndose serias dificultades en la manejabilidad del proceso de consolidación de los perfiles. Al fundir los tapes, éstos goteaban dando una distribución heterogénea de las fibras.

Por el contrario, a partir de la matriz de rPET blue hubo suficiente estabilidad para consolidar más de 50 metros de perfil compuesto (Figura 5.8). El perfil se obtuvo en forma de pletina con las dimensiones de $15 \times 2 \mathrm{~mm}^{2}$. Una vez obtenidos los perfiles, se procedió a estudiar sus propiedades finales.

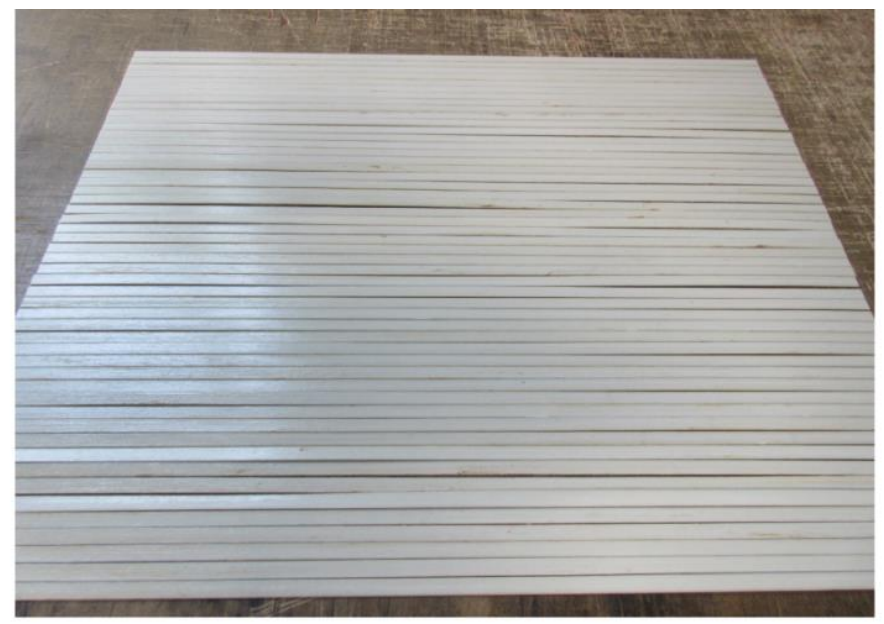

Figura 5.8 Imagen de los perfiles obtenidos por pultrusión en fundido (50 metros) 


\subsubsection{Caracterización de perfiles}

\section{Propiedades mecánicas}

\section{Flexión}

En primer lugar, se realizó el ensayo de flexión de los perfiles obtenidos con rPET blue como indica la Figura 5.9. Los perfiles elaborados por esta tecnología de pultrusión no llegaron a romper cuando fueron ensayados a flexión, sino que, llegan a recuperar parcialmente la posición inicial cuando se dejaba de ejercer la fuerza de deformación. Cuando el perfil se dobla, las fibras forman arcos circulares concéntricos, de modo que las fibras superiores (dentro de la curvatura) se comprimen y las inferiores (fuera de la curvatura) se estiran, rompiendo algunas de ellas, sin llegar a producirse la rotura total del perfil. Este comportamiento puede ser debido a que la impregnación de las fibras no haya sido totalmente efectiva, permitiendo que las fibras tengan un movimiento más libre.
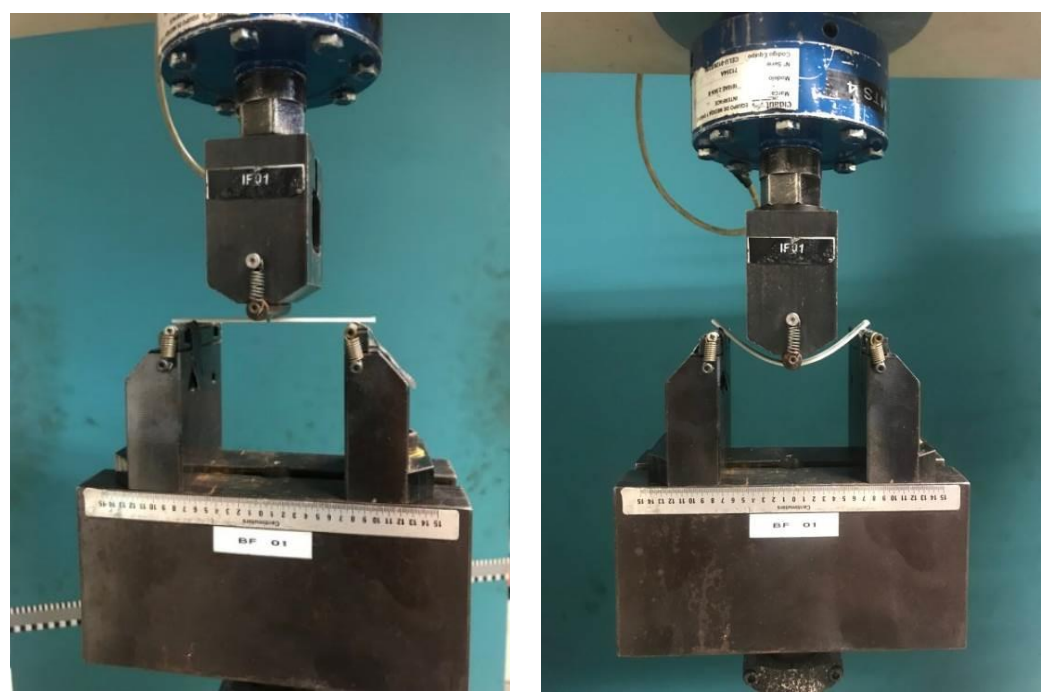

Imagen 5.9 Ensayo de flexión de perfil obtenido por pultrusión en fundido

A continuación, en la Tabla 5.2 se muestran las propiedades de flexión, donde se incluyen los resultados de 5 referencias que corresponden con distintas zonas del perfil de 50 metros. Los valores específicos son los cocientes de los valores experimentales y la fracción de volumen de fibra del perfil obtenida por calcinación $(67 \%)$. 
Tabla 5.2 Resultados de ensayo de flexión del perfil obtenido por pultrusión en fundido

\begin{tabular}{ccc}
\hline $\begin{array}{c}\text { No } \\
\text { Probeta }\end{array}$ & $\begin{array}{c}\text { Módulo de flexión } \\
\text { (GPa) }\end{array}$ & $\begin{array}{c}\text { Resistencia a la flexión } \\
\text { (MPa) }\end{array}$ \\
\hline $\mathbf{1}$ & 22.5 & 453 \\
$\mathbf{2}$ & 23.3 & 433 \\
$\mathbf{3}$ & 22.5 & 452 \\
$\mathbf{4}$ & 28.2 & 526 \\
$\mathbf{5}$ & 22.3 & 438 \\
\hline Media & $23.8 \pm 2.5$ & $461 \pm 37$ \\
\hline Específico & $35.5 \pm 3.7$ & $688 \pm 55$ \\
\hline
\end{tabular}

Los resultados mostraron que el uso de rPET blue como matriz para la producción de perfiles a partir de esta tecnología de pultrusión resultó con excelentes propiedades mecánicas de flexión.

\section{Tracción}

El siguiente ensayo mecánico que se realizó de los perfiles fue el de tracción que se muestra en la Figura 5.10. Es importante resaltar que la resistencia a la tracción de los perfiles compuestos (matriz polimérica y fibra) es más difícil de determinar que las de los componentes por sí solos. Esto se debe a que, al aplicar un esfuerzo de tracción al perfil, éste se distribuye en todas las fibras, aunque no de forma uniforme $y$, cuando el valor del esfuerzo de tracción se aproxima a la máxima resistencia de rotura, algunas fibras fallan y otras permanecen unidas sin llegar a la rotura.

En la Tabla 5.3 se muestran los resultados del ensayo de tracción, realizado también en 5 referencias de distintas zonas de los perfiles.

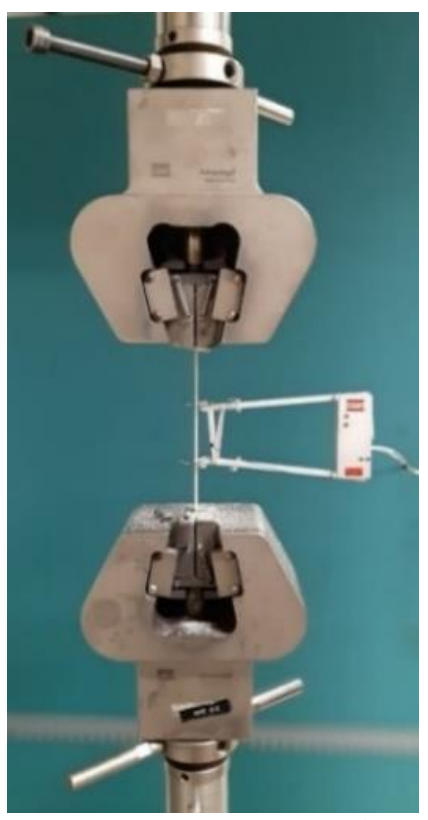

Figura 5.10 Ensayo de tracción de perfil (pultrusión en fundido) 
Tabla 5.3 Resultados del ensayo de tracción del perfil (pultrusión en fundido)

\begin{tabular}{ccc}
$\begin{array}{c}\text { No } \\
\text { Probeta }\end{array}$ & $\begin{array}{c}\text { Módulo de elasticidad } \\
\text { (GPa) }\end{array}$ & $\begin{array}{c}\text { Resistencia a tracción } \\
\text { (MPa) }\end{array}$ \\
\hline $\mathbf{1}$ & 37.9 & 519 \\
$\mathbf{2}$ & 37.7 & 465 \\
$\mathbf{3}$ & 39.9 & 432 \\
$\mathbf{4}$ & 37.4 & 422 \\
$\mathbf{5}$ & 37.8 & 560 \\
\hline Media & $38.1 \pm 0.9$ & $479 \pm 67$ \\
\hline Específico & $56.8 \pm 1.3$ & $714 \pm 100$ \\
\hline
\end{tabular}

Tanto en el ensayo de tracción como de flexión se obtuvieron resultados adecuados validando así el uso de PET $100 \%$ reciclado como matriz para la fabricación de composites termoplásticos bajo esta tecnología de pultrusión en fundido.

\section{Microscopía electrónica de barrido (SEM)}

En la Figura 5.11 se muestran las micrografías del perfil pultruido. Al observar la sección transversal mediante SEM, se puede ver como las fibras se encuentran compactadas con una buena impregnación de la matriz entre ellas. Sin embargo, aunque el polímero fundido penetró entre las fibras, se aprecian espacios vacíos a lo largo del espesor del perfil. Esto puede deberse a que el material no tuviese una viscosidad adecuada, sin embargo, como se vio anteriormente, esta tecnología no admitió trabajar con matrices más fluidas como aquellas obtenidas con los tratamientos químicos.

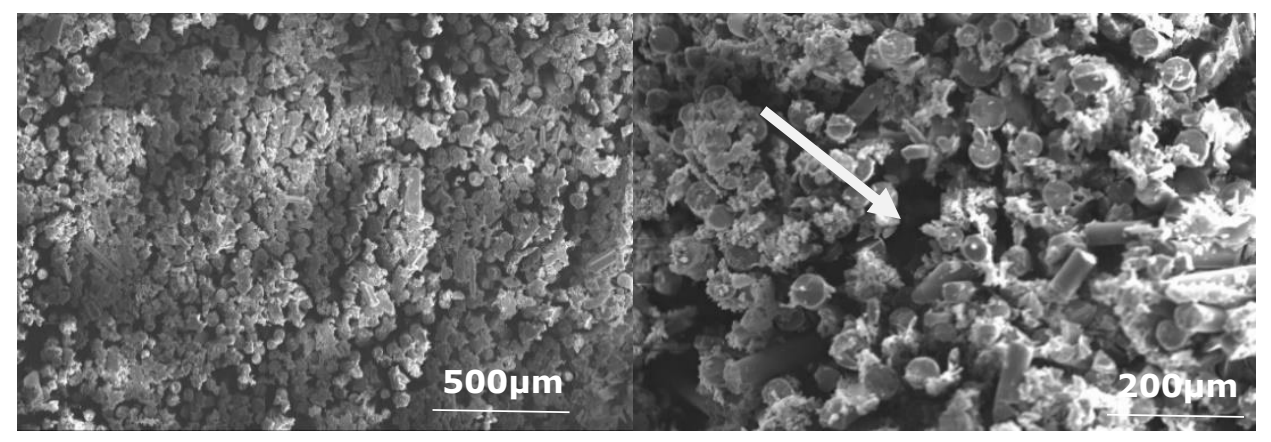




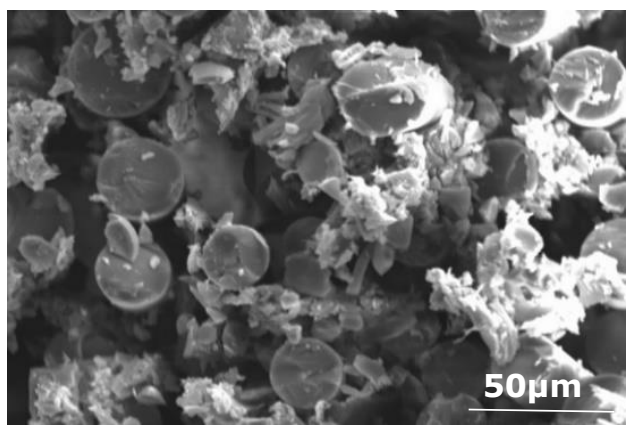

Figura 5.11 Micrografías del perfil (pultrusión en fundido)

\subsection{PULTRUSIÓN EN POLVO}

\subsubsection{Materiales}

En esta tecnología de pultrusión se utilizaron tres matrices de PET reciclado que se diferencian en el origen del residuo y en su viscosidad. En la Tabla 5.4 se muestran las matrices utilizadas para la fabricación de los perfiles por pultrusión en polvo y las propiedades más importantes de éstas que se han de tener en cuenta para el análisis de los resultados.

La matriz denominada PET 1 está formada por la corriente rPET blue y el PET 2 corresponde al rPET blue sometido a la modificación reológica mediante el método químico (rPET_0.25\%). Además de estos, en este proceso se introdujo el PET 3, que proviene de las corrientes residuales más contaminadas con presencia de materiales multicapa y coloreados (rPET color). Como se explicó en el Capítulo anterior, la introducción de estos residuos de PET actualmente no revalorizados (como el rPET color) en procesos de alta demanda como la pultrusión, es otro de los grandes retos de este trabajo.

Tabla 5.4 Propiedades de matrices de PET utilizadas en la pultrusión en polvo

\begin{tabular}{cccc}
\hline Propiedad & $\begin{array}{c}\text { rPET } \\
(\text { PET 1) }\end{array}$ & $\begin{array}{c}\text { rPET_0.25\% } \\
\text { (PET 2) }\end{array}$ & $\begin{array}{c}\text { rPET color } \\
\text { (PET 3) }\end{array}$ \\
\hline Corriente de PET & rPET blue & rPET blue & rPET color \\
\hline Densidad $\left(\mathrm{g} / \mathrm{cm}^{3}\right)$ & $1.326 \pm 0.007$ & $1.326 \pm 0.007$ & $1.324 \pm 0.004$ \\
Módulo de Young $(\mathrm{MPa})$ & $2369 \pm 36$ & $2427 \pm 19$ & $2579 \pm 84$ \\
& & & $26712 \pm 453$ \\
\hline
\end{tabular}


Como se ha explicado anteriormente, la viscosidad de las matrices poliméricas está relacionada con el grado de impregnación de la fibra y la matriz ${ }^{5,6}$. Por lo que para el proceso de pultrusión, es importante estudiar la viscosidad del PET reciclado durante el procesamiento, para comprender mejor su comportamiento. En general, la fabricación de materiales compuestos mejora con una menor viscosidad de las matrices en el estado fundido con la que se obtiene una mejor impregnación. Por lo que, a continuación, en la Figura 5.12, se representan los valores de viscosidad en fundido frente a la temperatura.

En la Figura se señala con una flecha vertical la temperatura a la que se obtuvieron los perfiles por pultrusión $\left(280^{\circ} \mathrm{C}\right)$. Las flechas horizontales señalan a esa temperatura, la viscosidad que van a presentar los materiales. La fibra de vidrio que se utilizó se detalla en la Tabla 5.1 en la sección de materiales.

Es importante señalar que los materiales de rPET utilizados para la pultrusión mostraron diferentes propiedades reológicas. EI PET 1 (que proviene del rPET blue) presenta una mayor viscosidad compleja que el PET 3 (rPET color). Como puede observarse, el PET 2 (material modificado químicamente) alcanzó valores de baja viscosidad similares a los del PET 3. En el comportamiento reológico de este material modificado se puede resaltar la baja viscosidad que presenta a bajas temperaturas $\left(265^{\circ} \mathrm{C}\right)$, lo que permitiría emplear a éste en procesos a más baja temperatura.

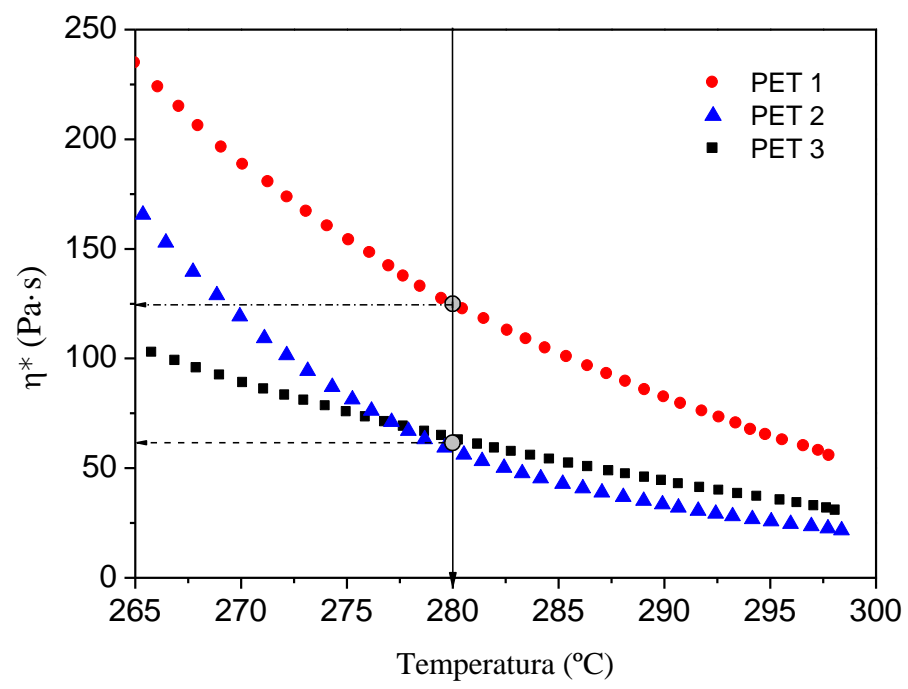

Figura 5.12 Viscosidad compleja de las matrices de rPET utilizadas en la pultrusión en polvo 


\subsubsection{Obtención de towpreg (pre-impregnado)}

\section{Reducción del tamaño de partícula}

Como se explicó anteriormente, esta metodología de pultrusión se diferencia de la anterior (en fundido) en la manera de fabricación del pre-impregnado; en especial, por el empleo de la matriz en forma de polvo. Con la reducción del tamaño de partícula se consigue una impregnación con la fibra homogénea y eficiente, por tener mayor área superficial en contacto; sin embargo, requiere de un paso previo para la obtención del tamaño de partícula adecuado.

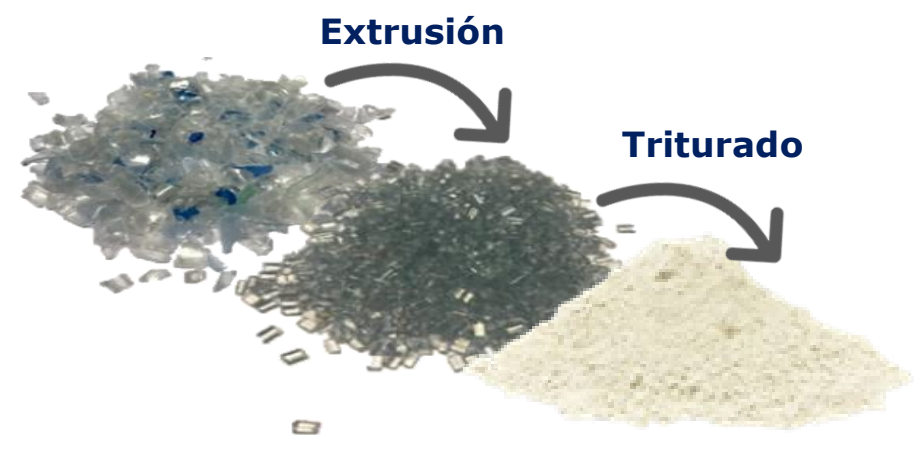

Figura 5.13 Imagen de la reducción del tamaño de partícula

Las matrices obtenidas de la tabla anterior en forma de granza, fueron

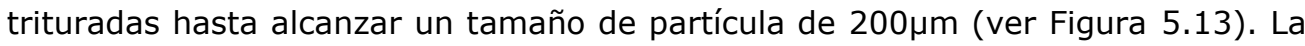
reducción del tamaño de partícula fue llevada a cabo por la empresa Powderplastic en líneas de triturado, a partir de molinos criogénicos. Para obtener una homogeneidad en el tamaño de partícula el material fue tamizado con un filtro de $200 \mu \mathrm{m}$.

\section{Obtención del towpreg}

El primer paso del proceso de pultrusión en polvo es la elaboración del preimpregnado (towpreg). El towpreg se produjo en la máquina de recubrimiento de polvo semi-industrial desarrollada e instalada en el Laboratorio de Materiales Compuestos en ISEP7,8 (detallada en el Capítulo 3). Este equipo permite la fabricación de towpreg, material pre-impregnado en el que el polímero en polvo tiene un contacto íntimo con las fibras. De forma más detalla, en la Figura 5.14 se muestra el principio de impregnación de este proceso, donde a la izquierda se ve cómo entra la fibra de vidrio caliente, sin impregnar, en el dispositivo que contiene el material en forma de polvo. A continuación, a la derecha se muestra cómo sale la fibra con el contenido de polvo, sobre ella. 

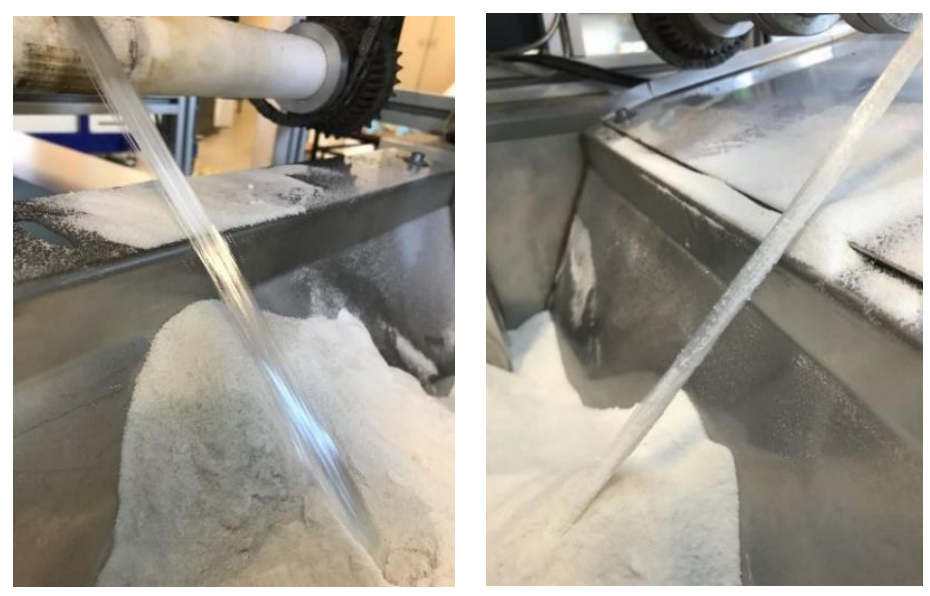

Figura 5.14 Imagen de impregnación de la fibra con el polvo

Después del estudio de los parámetros óptimos para los materiales estudiados, se encontró que la velocidad de fabricación del material pre-impregnado debía ser de seis metros por minuto, con el primer horno de convección configurado a $700^{\circ} \mathrm{C}$ y el segundo horno a $450^{\circ} \mathrm{C}$. Estas condiciones de fabricación fueron ajustadas hasta conseguir un towpreg con un contenido de matriz superior a un $45 \%{ }^{9}$.

Para la fabricación de los perfiles fue necesario producir mil metros de towpreg de cada matriz. El towpreg era enrollado en pequeñas bobinas de aproximadamente 30 metros y colocados en el dispositivo de barras para su posterior pultrusión. En la Figura 5.15 se muestra la forma física del towpreg obtenido, así como una visualización de SEM del estado de las fibras y la matriz.
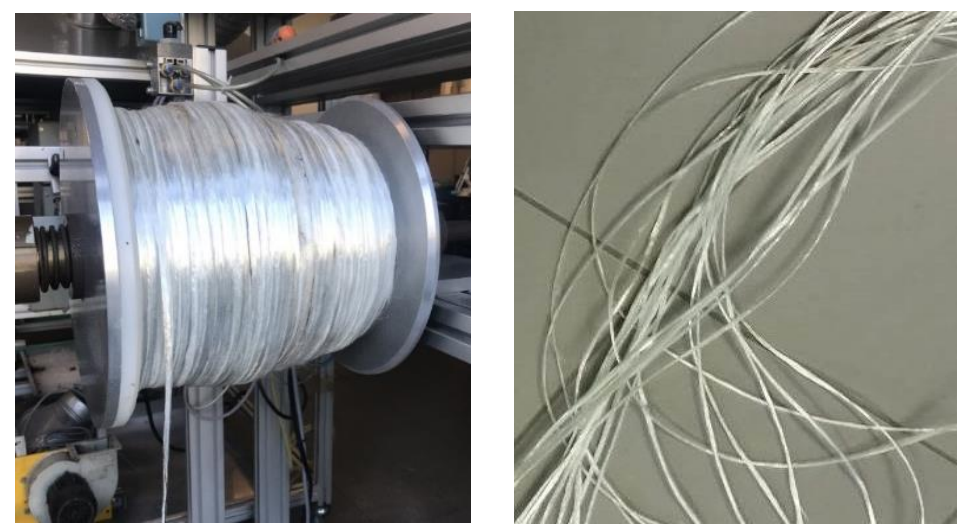


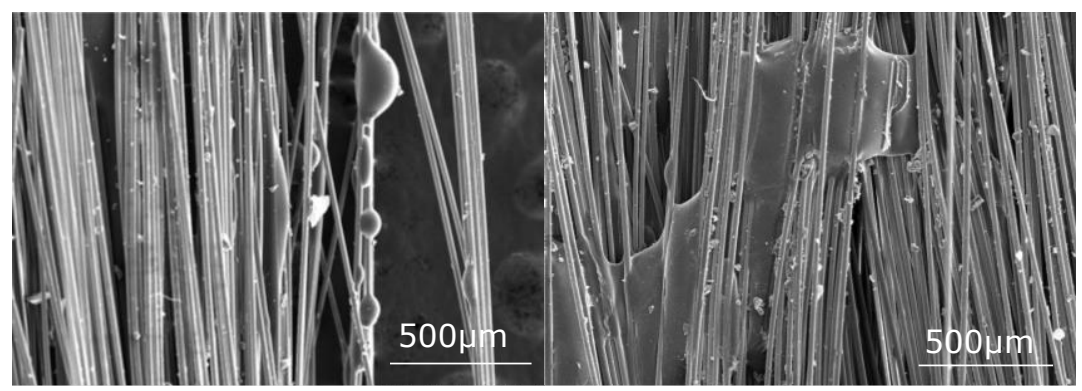

Figura 5.15 Imagen de towpreg (pre-impregnado) y de la inspección por SEM del towpreg

\subsubsection{Obtención de perfiles}

Una vez obtenidos los towpreg con las tres matrices de rPET, se llevó a cabo la pultrusión de éstos a una velocidad de 0.2 metros por minuto, introduciendo un número de 25 towpregs. El primer horno de precalentamiento se configuró a $180^{\circ} \mathrm{C}$. La matriz de presurización y consolidación se ajustó a $280^{\circ} \mathrm{C}$, y el molde de enfriamiento fue refrigerado a $25^{\circ} \mathrm{C}$. El molde de consolidación utilizado en este proceso permitió la fabricación de un perfil compuesto con forma de pletina de $20 \times 2$ $\mathrm{mm}^{2}$ (Figura 5.16).

El color amarillento que se observa en los perfiles se debe a la presencia de grupos éster de vinilo que se generan en las reacciones de degradación térmica que experimenta el PET en cualquiera de sus modificaciones durante el procesado (reacciones mostradas en el Capítulo 4) 10,11.
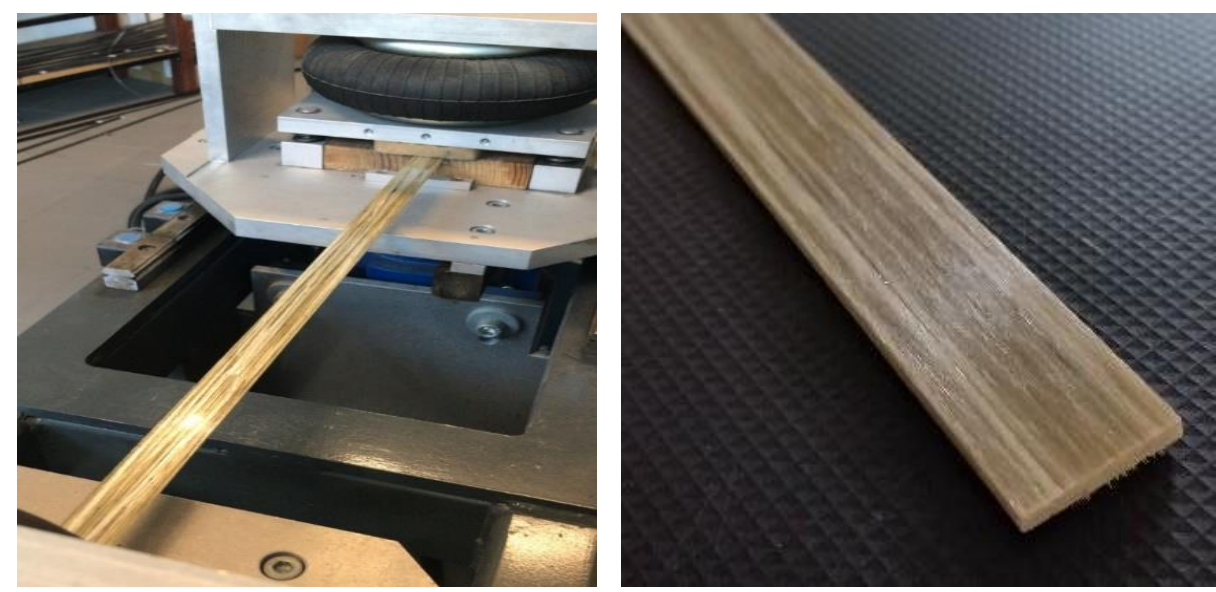

Figura 5.16 Perfiles obtenidos por pultrusión en polvo 


\subsubsection{Caracterización de los perfiles}

\section{Caracterización mecánica}

\section{Flexión}

Una vez obtenidos los perfiles por el proceso de pultrusión en polvo, se llevó a cabo la caracterización mecánica. La Tabla 5.5 muestra las propiedades de flexión de los perfiles desarrollados y los valores relativos de las propiedades específicas. Además, se muestra la fracción de volumen de fibra obtenida por calcinación.

Tabla 5.5 Propiedades de flexión de los perfiles (pultrusión en polvo)

\begin{tabular}{|c|c|c|c|}
\hline & \multirow{2}{*}{\multicolumn{3}{|c|}{ Pultrusión }} \\
\hline & & & \\
\hline Propiedad & $\begin{array}{l}\text { Towpreg } \\
\text { FV/PET } 1\end{array}$ & $\begin{array}{l}\text { Towpreg } \\
\text { FV/PET } 2\end{array}$ & $\begin{array}{l}\text { Towpreg } \\
\text { FV/PET } 3\end{array}$ \\
\hline Módulo de flexion (GPa) & $40.7 \pm 0.5$ & $45.1 \pm 1.0$ & $38.3 \pm 0.9$ \\
\hline Módulo de flexión específico (GPa) & $70.2 \pm 0.9$ & $70.5 \pm 1.6$ & $60.1 \pm 1.4$ \\
\hline Resistencia a flexión (MPa) & $773 \pm 48$ & $1067 \pm 21$ & $594 \pm 49$ \\
\hline Resistencia a flexión específica (MPa) & $1207 \pm 74$ & $1662 \pm 32$ & $932 \pm 76$ \\
\hline Fracción de volumen de fibra (\%) & 64.1 & 64.2 & 63.7 \\
\hline
\end{tabular}

Como se puede apreciar en la Tabla, el PET 1 y el PET 2 presentaron valores específicos de Módulo de flexión similares. Por lo que, en este caso, la diferencia en viscosidad que presentan no influye en las propiedades a flexión del perfil. En cambio, como se puede ver, PET 3 mostró valores más bajos de Módulo de flexión. Esta disminución puede deberse al hecho de que el PET 3 proviene de una corriente reciclada con algunas contaminaciones que pudiesen actuar como puntos de fallo en la impregnación ${ }^{12,13}$. Estos residuos de PET coloreados han sido estudiados por otros autores, donde observaron que los contaminantes que contiene el rPET color hacen disminuir las propiedades mecánicas, mostrando valores de Módulo de flexión más bajos que el PET que proviene de residuos de botellas azules ${ }^{14}$. 
En cuanto a la Resistencia a la flexión específica, el perfil obtenido con PET 2 (rPET blue modificado químicamente) mostró los valores más altos. Esto se debe a que la baja viscosidad que presenta el PET 2 (ver Figura 5.12) permite un mejor recubrimiento de las fibras $y$, por consiguiente, una mejor unión entre las fibras y la matriz ${ }^{15}$, una mejor consolidación de los perfiles finales y a la influencia de los grupos funcionales injertados, que no solamente han mejorado la mojabilidad sino la interacción química polímero-matriz.

Por otra parte, el perfil obtenido con PET 3 (rPET color) mostró el valor más bajo de Resistencia a flexión (a pesar de tener valores de viscosidad bajos). Esto se debe, como se ha explicado anteriormente, a que la presencia de contaminantes en este material dificulta la impregnación y consolidación, y consecuentemente, empeoran las propiedades mecánicas.

En la Figura 5.17 podemos ver la imagen del perfil tras haber sido ensayado a flexión. Se puede ver cómo las fibras de dentro de la curvatura han sido comprimidas, y las fibras de fuera han sido traccionadas hasta llegar a la rotura casi total del perfil. A diferencia de los perfiles obtenidos por pultrusión en fundido que recuperan parcialmente su estado inicial (deformación elástica), estos perfiles rompían sin recuperar su estado inicial. Este hecho corrobora que las fibras han sido impregnadas adecuadamente, la matriz está fuertemente adherida a la fibra lo que proporciona un comportamiento rígido al perfil impidiendo que pandee.

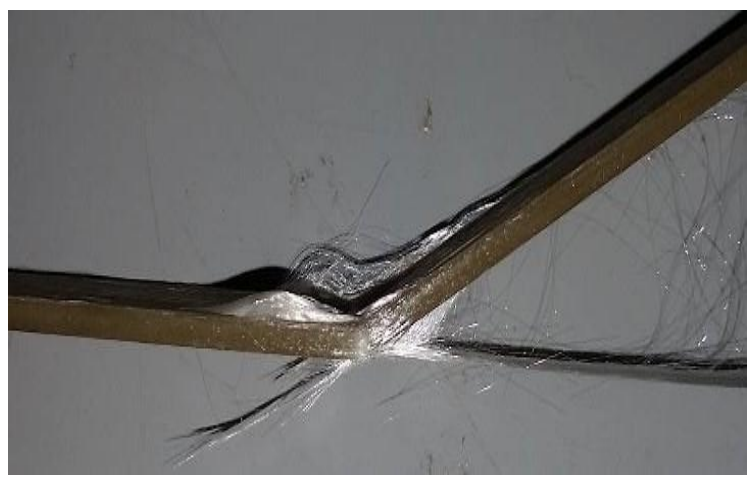

Figura 5.17 Rotura del perfil durante el ensayo de flexión 


\section{Tracción}

A continuación, se realizó el ensayo de tracción de los perfiles obtenidos con las matrices de rPET (Tabla 5.6). Los resultados mostrados en la Tabla son consecuentes con los ensayos de flexión: el perfil obtenido con la matriz de PET 2 (rPET_0.25\%) muestra mayor Módulo y Resistencia a la tracción y el perfil elaborado con la matriz de PET 3 presenta los valores más bajos de Módulo y Resistencia a la tracción. Aludiendo a las mismas razones anteriores: La modificación química provee el mejor mecanismo de regulación de la viscosidad, además de aumentar la compatibilidad química matriz-fibra. Por otra parte, las impurezas del reciclado (residuos coloreados y multicapa) pueden explicar las propiedades mecánicas más bajas.

Tabla 5.6 Propiedades de tracción de los perfiles (pultrusión en polvo)

\begin{tabular}{|c|c|c|c|}
\hline & \multirow{2}{*}{\multicolumn{3}{|c|}{ Pultrusión }} \\
\hline & & & \\
\hline Propiedad & $\begin{array}{l}\text { Towpreg } \\
\text { FV/PET } 1\end{array}$ & $\begin{array}{l}\text { Towpreg } \\
\text { FV/PET } 2\end{array}$ & $\begin{array}{l}\text { Towpreg } \\
\text { FV/PET } 3\end{array}$ \\
\hline Módulo de elasticidad (GPa) & $47.3 \pm 1.8$ & $50.0 \pm 0.9$ & $45.3 \pm 0.3$ \\
\hline Módulo específico (GPa) & $73.8 \pm 2.8$ & $77.8 \pm 1.4$ & $71.1 \pm 0.4$ \\
\hline Resistencia a la tracción (MPa) & $351 \pm 51$ & $441 \pm 23$ & $243 \pm 33$ \\
\hline Resistencia específica (MPa) & $548 \pm 80$ & $688 \pm 37$ & $382 \pm 53$ \\
\hline Fracción de volumen de fibra (\%) & 64.1 & 64.2 & 63.7 \\
\hline
\end{tabular}

\section{Microscopía electrónica de barrido (SEM)}

Para demostrar esta mejora en la impregnación, en la siguiente Figura 5.18 se muestran las micrografías obtenidas por SEM de los perfiles obtenidos mediante el proceso de pultrusión en polvo. Las micrografías se tomaron en la zona de la fractura de los perfiles ensayados a flexión (Figura 5.17). Como se comentó anteriormente, las fibras deben estar firmemente unidas a la matriz para que la carga pueda transferirse correctamente de la matriz a las fibras. Si la unión es pobre, las fibras pueden separarse de la matriz durante la carga o esfuerzo, reduciendo la resistencia a la fractura del perfil compuesto. A través de las micrografías podemos comprender algunas de las propiedades mecánicas que han presentado los perfiles. 

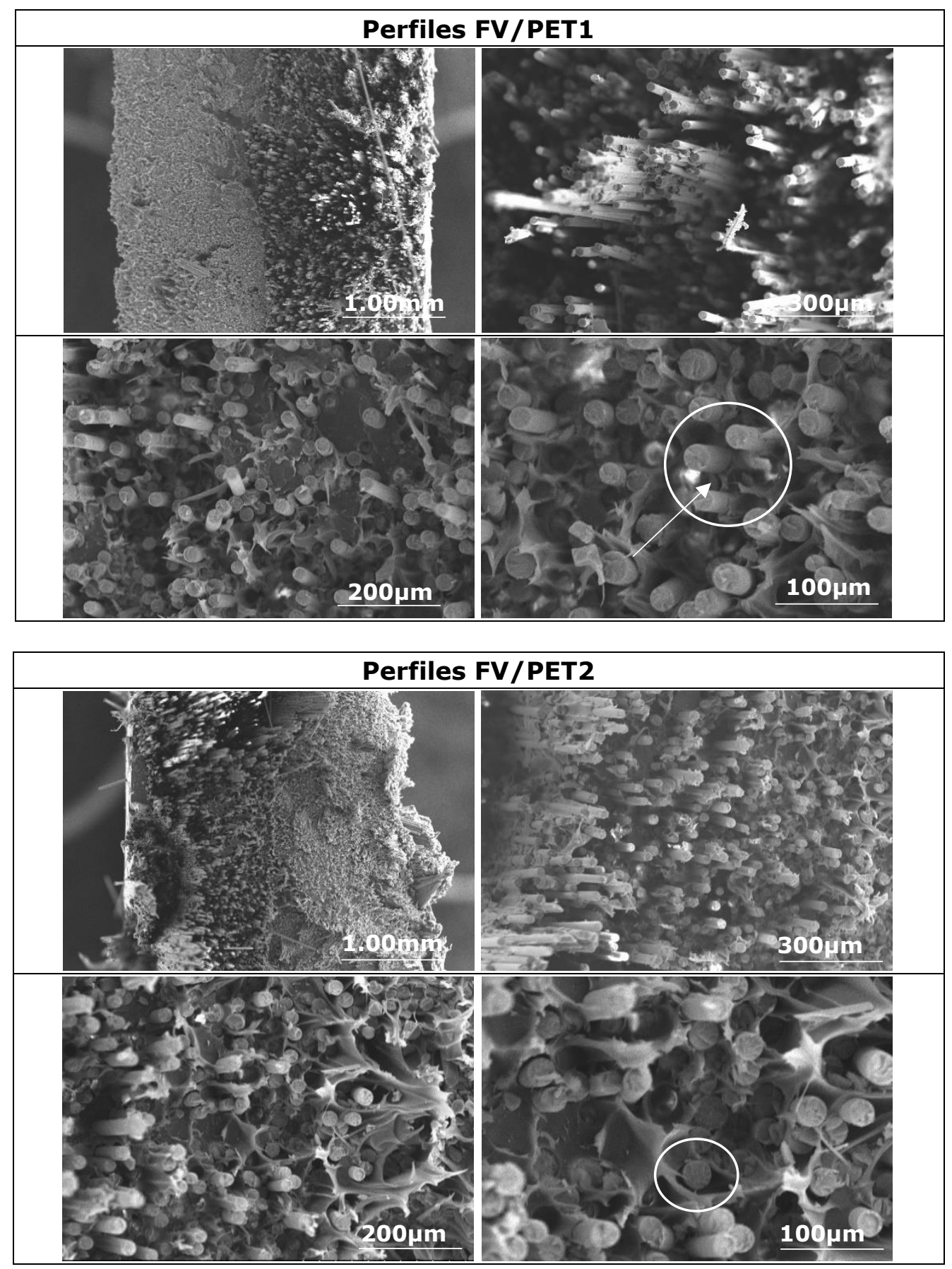


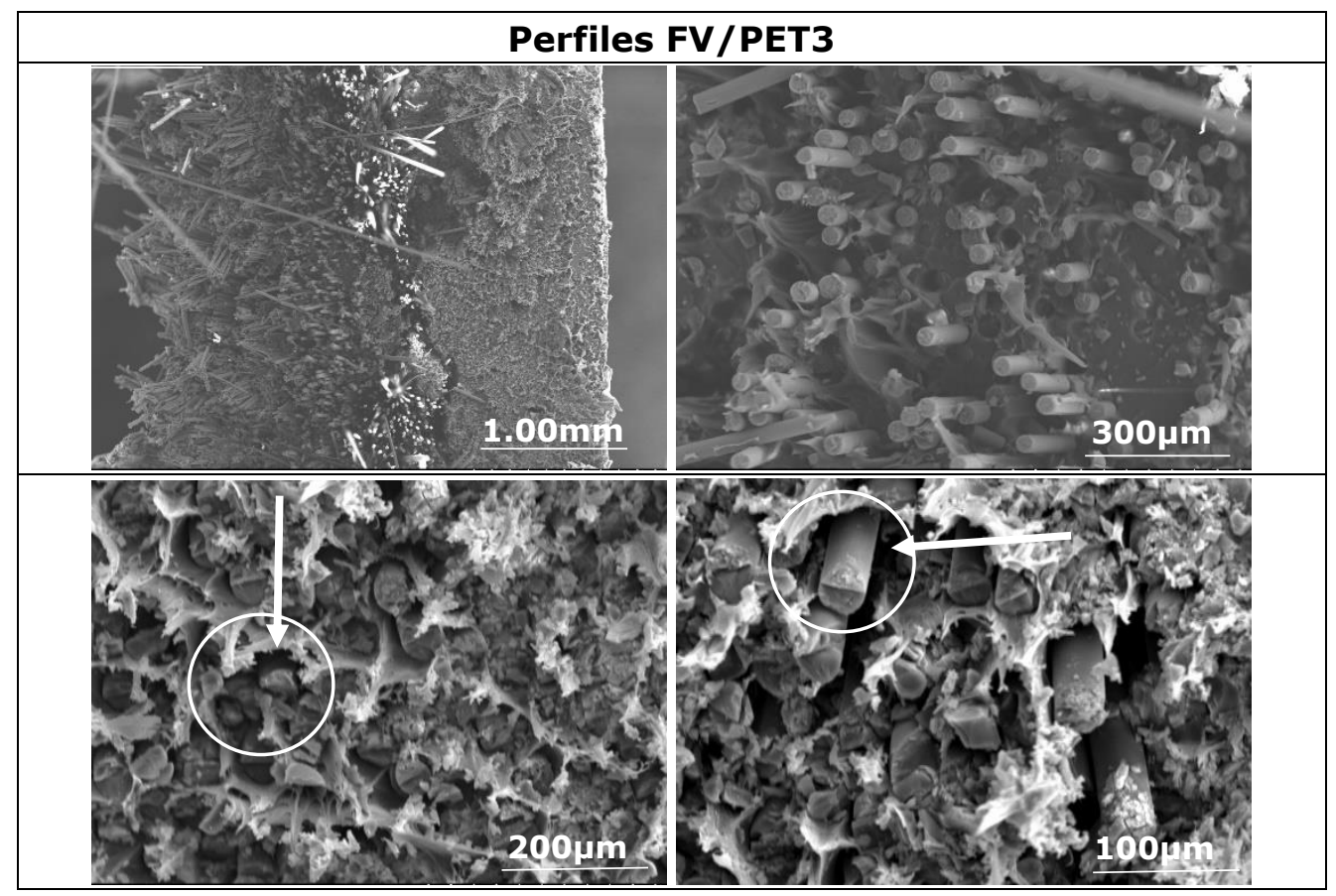

Figura 5.18 Imágenes de SEM de perfiles obtenidos (pultrusión en polvo)

Como se puede apreciar, a partir de la utilización de las tres matrices de PET reciclado se consiguen perfiles con una adecuada impregnación. Sin embargo, se observan diferencias importantes en la calidad de la impregnación entre las distintas matrices.

Los perfiles obtenidos con PET 1 y PET 2, que corresponden con la corriente rPET blue, mostraron una buena impregnación, presentándose las fibras completamente rodeadas por el polímero (ver círculos señalando las fibras embebidas en la matriz). Por el contrario, el PET 3 presentaba zonas con la matriz sin adherirse a la fibra y algunos vacíos alrededor de la fibra. Estos vacíos se pueden ver en la micrografía del PET 3 señalados con una flecha. Además, se pueden observar zonas sin fibras donde se ha dado una distribución menos homogénea de matriz-fibra. Los resultados de la microscopía concuerdan con la caracterización mecánica, donde el PET 3 mostró los valores más bajos en las propiedades mecánicas. 


\subsection{COMPARACIÓN CON MATRICES TERMOESTABLES CONVENCIONALES}

Como se explicó anteriormente, los procesos de pultrusión convencionales emplean matrices termoestables (pultrusión termoestable), como son, en orden a su volumen de utilización, las resinas de poliéster, viniléster, acrílicas, epóxicas y fenólicas. El $80 \%$ de los productos pultrusionados se forman actualmente a partir de resina de poliéster, PET virgen en su tipo isoftálico, ya que posee elevadas propiedades de resistencia química y mecánica que se requieren en los productos de uso más frecuente: suelos industriales, tanques y tuberías, construcción, electricidad, industria marítima y automotriz. Las otras variedades se utilizan según las características especiales que demanda la utilización del perfil a fabricar.

En las siguientes Figuras se comparan las propiedades mecánicas específicas de los perfiles termoplásticos obtenidos en esta investigación a partir de rPET con los valores específicos de las propiedades mecánicas de estos perfiles termoestables usualmente utilizados (poliéster en su tipo isoftálico) ${ }^{16}$.

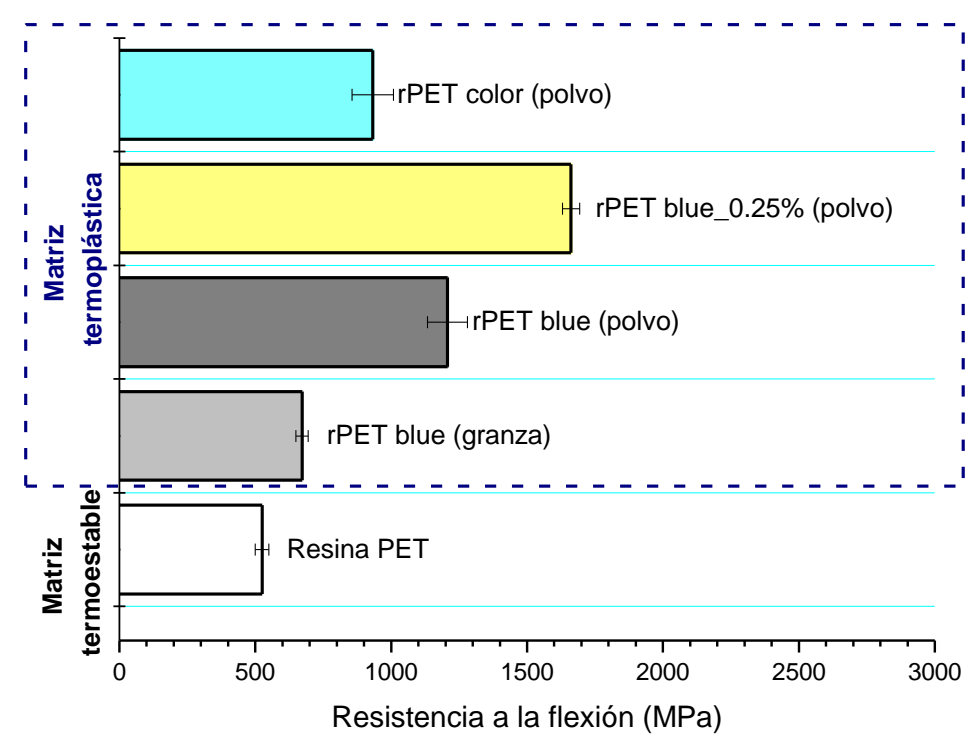

Figura 5.19 Comparación de Resistencia a la flexión de perfil termoestable convencional y perfiles termoplásticos obtenidos en la investigación con rPET 


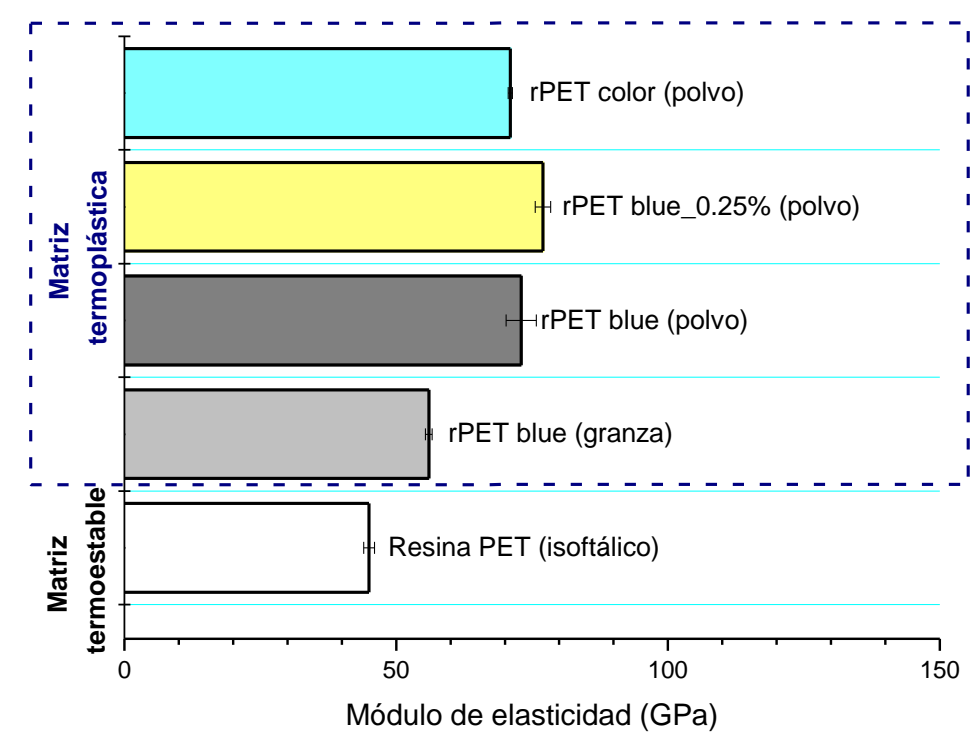

Figura 5.20 Comparación de Módulos elásticos de un perfil termoestable convencional y perfiles termoplásticos obtenidos en esta investigación con rPET

Como se puede ver en las Figuras 5.19 y 5.20, los perfiles elaborados en esta investigación con las matrices termoplásticas de rPET alcanzaron mayores valores específicos de Resistencia a flexión y Módulo de elasticidad que un perfil termoestable convencional. Lo que demuestra la validez de estos perfiles para su uso en aplicaciones similares a las que se emplean con resinas de PET virgen isoftálica.

Si comparamos los procesos de pultrusión empleados en esta investigación (granza y polvo), se puede observar que a partir de la misma matriz (rPET blue) se obtienen mejores propiedades de Resistencia a flexión y Módulo de elasticidad a partir de la pultrusión en polvo. Esto, posiblemente esté asociado a los efectos de la distribución de la matriz polimérica en los pre-impregnados, permitiendo a partir del towpreg alcanzar mayor adhesión de la matriz a la fibra. Como se pudo observar en el análisis microscópico, el perfil obtenido por pultrusión en fundido presentó algunos vacíos alrededor de la fibra. Sin embargo, como contraparte de este proceso, se requiere de un paso adicional (triturado de material granzaspolvo). 
A continuación, en la Figura 5.21 se representan los valores de Resistencia a tracción específicos donde se puede apreciar como todos los perfiles desarrollados en esta investigación, excepto el rPET color, superaron las propiedades a tracción del perfil termoestable convencional.

Además, se puede observar como en este caso, el perfil elaborado por pultrusión en fundido presenta mayor Resistencia a la tracción que el perfil obtenido por el proceso de pultrusión el polvo. Este hecho puede deberse a que en el ensayo de tracción las fibras ejercen el papel más importante, por lo que, por la tecnología de pultrusión en fundido se obtienen perfiles con las fibras más homogéneamente ordenadas ejerciendo mayores valores de Resistencia a la tracción.

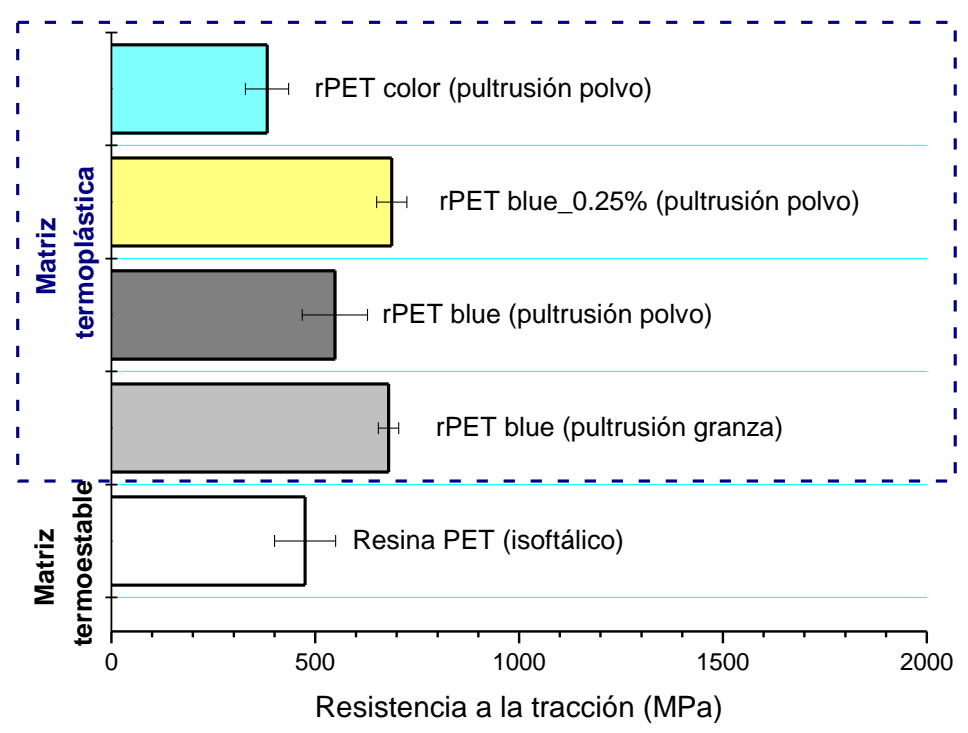

Figura 5.21 Comparación de Resistencia a la tracción de perfil termoestable convencional y perfiles termoplásticos obtenidos en la investigación con rPET

\subsection{CONCLUSIONES}

En primer lugar, cabe destacar que el diseño, construcción y puesta a punto de la cámara de impregnación nos permitió, de una manera rápida, valorar si las modificaciones realizadas sobre las matrices mejoraban el proceso de impregnación. Como resultado, se pudo observar que el método de modificación química resultó ser el más eficaz para este objetivo. 
Una de las conclusiones más importantes de este capítulo ha sido la posibilidad de obtener perfiles pultruidos que estén compuestos en su totalidad de material de PET reciclado, utilizando las dos tecnologías de pultrusión (en fundido y en polvo).

El proceso de pultrusión en fundido, permitió obtener perfiles con buenas propiedades mecánicas a partir de rPET blue en un proceso industrializado. En este proceso también se estudió la posibilidad de introducir un material reciclado de PET modificado químicamente (rPET_0.25\%), donde se detectó cómo la viscosidad baja de las matrices imposibilitaba la producción de perfiles por este proceso de pultrusión en fundido. En cambio, en el proceso de pultrusión en polvo (semiindustrial), se pudo ver que, cuanto más baja era la viscosidad mejores propiedades mecánicas presentaba el perfil.

El proceso de pultrusión en polvo permite concluir que es posible producir, en buenas condiciones, perfiles a partir de los tres materiales PET reciclados. Este estudio nos permitió conocer que las diferencias principales en las propiedades de los perfiles radican en el diferente origen del material reciclado y en la viscosidad de las matrices. Los materiales de la corriente de rPET blue (PET 1 y PET 2) utilizados para la fabricación de perfiles mostraron mayor facilidad de fabricación y mejores propiedades mecánicas. Además, de poder concluir que, a partir del material desarrollado con valores de viscosidad más bajos (PET 2) se consigue obtener una mejor impregnación $y$, en consecuencia, perfiles con propiedades mecánicas mejoradas.

Por otro lado, el proceso de pultrusión en polvo permitió utilizar el rPET color como materia prima obteniendo propiedades mecánicas como Módulo de elasticidad y resistencia a flexión superiores a un perfil termoestable. Una conclusión importante del uso de este material como matriz está asociada a sus propiedades, que debido a la contaminación que presentan como son los multicapa y materiales coloreados, provocan que las propiedades sean peores que un material rPET más limpio. En este caso, el logro de obtener un perfil compuesto a partir de este material es muy interesante, ya que actualmente no se recicla el material coloreado en ninguna aplicación de alto valor y larga vida útil.

Finalmente, la caracterización mecánica que se ha llevado a cabo de los perfiles (en fundido y en polvo) permite predecir el uso adecuado del PET reciclado en procesos de pultrusión, ya sea para su uso en aplicaciones generales o de ingeniería estructural. 


\subsection{REFERENCIAS BIBLIOGRÁFICAS}

1. Lapointe F, Lebel LL. Fiber Damage and Impregnation during Multi-Die Vacuum Assisted Pultrusion of Carbon / PEEK Hybrid Yarns. Polym Compos. 2019;40(S2):E1015-E1028. doi:10.1002/pc.24788

2. De Moura Giraldi ALF, De Jesus RC, Mei LHI. The influence of extrusion variables on the interfacial adhesion and mechanical properties of recycled PET composites. J Mater Process Technol. 2005;162-163(SPEC. ISS.):90-95. doi:10.1016/j.jmatprotec.2005.02.046

3. Mitschang P, Christmann M. Continuous Fiber Reinforced Profiles in Polymer Matrix Composites. Woodhead Publishing 2012. doi:10.1533/9780857096258.2.209

4. Novo PJ, Silva JF, Nunes JP, Van Hattum FWJ, Marques AT. Development of a new pultrusion equipment to manufacture thermoplastic matrix composite profiles. ECCM 2012 - Compos Venice, Proc 15th Eur Conf Compos Mater. 2012;(June):18-25.

5. Wilson ML, Buckley JD. The Potential for Low Cost Thermoplastic Pultrusion. J Reinf Plast Compos. 1994;13(10):927-941. doi:10.1177/073168449401301007

6. Hedayati H, Mohammad V. Composites based on HDPE, jute fiber, wood, and thermoplastic starch in tubular pultrusion die: The correlation between mechanical performance and microstructure. Adv Polym Technol. 2018;37(July):3483-3491. doi: 10.1002/adv.22132

7. Silva FJG, Amorim E, Baptista A, Pinto G, Campilho RDSG, Castro MRA. Producing hybrid pultruded structural products based on preforms. Compos Part B Eng. 2017;116:325-332. doi:10.1016/j.compositesb.2016.10.070

8. João Silva, João Nunes, C. A. Bernardo AM. Thermoplastic Matrix Composites from Towpregs.; 2013. doi:http://dx.doi.org/10.5772/46845

9. Novo PJ, Silva JF, Nunes JP, Marques AT. Pultrusion of fibre reinforced thermoplastic pre-impregnated materials. Compos Part B Eng. 2016;89:328-339. doi: 10.1016/j.compositesb.2015.12.026

10. Besnoin JM, Choi KY. Identification and Characterization of Reaction Byproducts in the Polymerization of Polyethylene Terephthalate. J Macromol Sci Part C. 1989;29(1):5581. doi: $10.1080 / 07366578908055164$

11. Masmoudi F, Fenouillot F, Mehri A, Jaziri M, Ammar E. Characterization and quality assessment of recycled post-consumption poly(ethylene terephthalate) (PET). 2018;(June). doi:10.1007/s11356-018-2390-7

12. Cruz SA, Scuracchio CH, Fitaroni LB, Oliveira C. The use of melt rheology and solution viscometry for degradation study of post-consumer poly(ethylene terephthalate): The effects of the contaminants, reprocessing and solid state polymerization. Polym Test. 2017;60:236-241. doi:10.1016/j.polymertesting.2017.03.026

13. Torres N, Robin J], Boutevin B. Study of thermal and mechanical properties of virgin and recycled poly(ethylene terephthalate) before and after injection molding. Eur Polym J. 2000;36(10):2075-2080. doi:10.1016/S0014-3057(99)00301-8

14. Elamri A, Lallam A, Harzallah O, Bencheikh L. Mechanical characterization of melt spun fibers from recycled and virgin PET blends. J Mater Sci. 2007;42(19):8271-8278. doi:10.1007/s10853-007-1590-1

15. Luisier A, Bourban PE, Månson JAE. Reaction injection pultrusion of PA12 composites: Process and modelling. Compos Part A Appl Sci Manuf. 2003;34(7):583-595. doi:10.1016/S1359-835X(03)00101-5

16. Manufacturas de plásticos reforzados S.L. http://www.mplr.net/. 


\section{Ca pítu I o 6}

Este último capítulo servirá para presentar las conclusiones más importantes que se derivan de esta investigación, así como el impacto y las perspectivas futuras que se plantean a partir de los resultados obtenidos.

\section{Conclusiones y perspectivas futuras}

\subsection{CONCLUSIONES}

\subsubsection{Conclusiones del trabajo de investigación}

Las conclusiones más relevantes obtenidas del presente trabajo sobre el efecto de la modificación reológica de los materiales de PET y la obtención de perfiles son las siguientes:

\section{Modificación reológica de los materiales}

- En todos los tratamientos aplicados para disminuir la viscosidad del PET (virgen o residual) y controlar su reología, se observó un fenómeno aditivo entre la termo-cizalla aplicada por el procesado (extrusión) y el tratamiento propuesto (hidrólisis, injerto químico y las mezclas).

- El estudio por separado del efecto de cada parámetro experimental del procesado por extrusión (temperatura, caudal y velocidad de husillo), demostró que estos son determinantes en el éxito de los tratamientos que se aplican en paralelo. Así mismo, la hidrólisis (escisión de cadenas en presencia de agua) no fue un tratamiento efectivo en la disminución de la viscosidad si no se aplicaba a altas temperaturas y cizallas, incluso en muchos casos, sin añadir el agua, estas condiciones severas de procesado conseguían un material con los pesos moleculares deseados.

- La modificación reológica mediante el método físico ha permitido disminuir los valores de viscosidad del rPET con la incorporación de un VPET de alta fluidez, sin repercutir en la perdida de propiedades mecánicas.

- La modificación reológica mediante la vía química ha permitido obtener valores de viscosidad inferiores a los del método físico. El aditivo 5 ácido amino isoftálico se inserta en la cadena polimérica de las matrices de PET (virgen y reciclado) y sus mezclas, introduciendo grupos aromáticos que proporcionan 
más rigidez al material y promueven, con ello, el incremento en mayor medida que el método físico de los valores del Módulo de Young.

- Estos métodos proporcionan al rPET una combinación mejorada de fluidez y Módulo elástico que permite el uso de presiones y temperaturas más bajas en la fabricación de composites por pultrusión. El método químico permite utilizar el $100 \%$ de rPET como matriz para la fabricación de composites por pultrusión mientras que el método físico permitiría el uso de un máximo del 50\%.

- El trabajo realizado en el diseño y puesta a punto de la cámara de impregnación permitió, de una manera rápida, valorar si las modificaciones realizadas sobre las matrices mejoraban el proceso de impregnación. Como resultado se pudo observar que el método de modificación química resultó ser el más eficaz para este objetivo.

\section{Obtención de perfiles}

- Una de las conclusiones más importantes de esta parte de la investigación ha sido la posibilidad de obtener perfiles pultruidos en los que su matriz esté compuesta en su totalidad por PET reciclado (rPETblue) mediante las dos tecnologías de pultrusión. Este hecho demuestra la posibilidad de reciclar el PET en productos de alto valor y larga vida útil como son los perfiles.

- El rPET blue ha sido introducido como materia prima en un proceso industrial de pultrusión en fundido, en el que se obtuvieron perfiles con buenas propiedades mecánicas. Este proceso presenta como ventaja el uso de forma directa de la matriz tras salir del proceso de extrusión (granza). La viscosidad baja de las matrices imposibilita la producción de perfiles por el proceso de pultrusión en fundido.

- El proceso semi-industrial de pultrusión en polvo, a partir del preimpregnado "towpreg", permite concluir que es posible producir en buenas condiciones perfiles a partir de los tres materiales de rPET seleccionados. Las diferencias principales en las propiedades de los perfiles radican en la viscosidad de las matrices y en el diferente origen del material reciclado:

- El uso del material desarrollado por el método químico con valores de viscosidad más bajos permite obtener una mejor impregnación y, en consecuencia, perfiles con propiedades mecánicas mejoradas. 
- Una conclusión importante del uso del rPET color como matriz polimérica está asociada a sus propiedades, que debido a la contaminación que presentan como los materiales multicapa y materiales coloreados, provocan que las propiedades sean peores que un material rPET más limpio. El logro de obtener un perfil compuesto a partir de este material es muy interesante ambientalmente, ya que actualmente no se recicla este tipo de materiales en ninguna aplicación de alto valor y larga vida útil.

- A partir de ambos procesos de producción, se obtuvieron perfiles de rPET (tanto el rPET blue como el color) con propiedades mecánicas de flexión superiores a las propiedades que se requieren en aplicaciones en las que actualmente se emplean matrices termoestables (resina de PET isoftálica). De esta manera, el reemplazo de estas resinas termoestables por matrices termoplásticas sería factible desde el punto de vista de las propiedades mecánicas, y con ello, no solo se introduciría un material reciclado (disminuyendo la utilización de recursos fósiles), sino que también se crearía un producto reciclable.

\subsubsection{Impacto}

El impacto esperado del presente trabajo de investigación constituye una aportación científica y técnica al conocimiento y aplicación de materiales termoplásticos en los procesos de pultrusión. La investigación fundamental científica y técnica surge de la necesidad de resolución de problemas planteados en la sociedad, enfocándose en la acción sobre el cambio climático y eficiencia en la utilización de recursos y materias primas. Además de desempeñar un papel importante en el movimiento hacia una economía circular.

Este trabajo de investigación tendrá un alto impacto científico puesto que se propone el desarrollo de una nueva generación de materiales termoplásticos con propiedades reológicas mejoradas para su implantación en la industria de la pultrusión. La aplicabilidad de los termoplásticos modificados en los diferentes sectores tecnológicos supondrá un gran impacto industrial ya que la mejora en las propiedades permitirá su uso en estructuras de altas prestaciones, tanto en el sector de la automoción como en otras áreas del transporte y de la construcción, mejorando la capacidad competitiva de las industrias que utilicen estos materiales.

La transición hacia los composites termoplásticos tendrá un gran impacto ambiental, gracias a sus beneficios sobre los termoestables. El control de la contaminación ambiental, el alto rendimiento y la oportunidad de reciclaje hacen que los compuestos termoplásticos sean muy atractivos, respondiendo a la demanda actual de materiales respetuosos con el medio ambiente. 


\subsection{PERSPECTIVAS FUTURAS}

- Desarrollo de materiales avanzados a partir de rPET color:

Los perfiles pultruidos a partir de rPET color, a pesar de presentar bajas viscosidades que favorecen la impregnación de las fibras, mostraron propiedades más bajas que los perfiles pultruidos de un rPET más viscoso (rPET blue). Una mejora de las propiedades de los perfiles de rPET color se conseguiría mediante la obtención de blends por compounding de rPET color y blue y así poder ampliar su rango de aplicación.

- Tratamientos de las fibras:

La naturaleza inorgánica de las fibras no es compatible con los grupos funcionales de los polímeros y por esta razón éstas son sometidas a tratamientos de compatibilización específicos (ensimaje) dependiendo de la matriz que se vaya a emplear. De hecho, las fibras que actualmente se comercializan han sido sometidas a tratamientos diseñados para compatibilizarlas con matrices termoestables. Un adecuado tratamiento de las fibras permitiría una mejor adhesión del PET, favoreciendo el proceso de impregnación y consecuentemente, de las propiedades finales del perfil.

- Termoformado de perfiles:

Los perfiles pultruidos termoplásticos, gracias a la capacidad de ser termoformados, permitirán la obtención de formas geométricas complejas no alcanzables por las matrices termoestables en los distintos procesos de producción. Además de poder ser termoformados, gracias a su capacidad de volver a fundir, estos materiales permiten la posibilidad de sobre-inyectar formas geométricas complicadas al mismo perfil con lo que se conseguiría anclar otras piezas funcionales.

- Reciclaje de composites:

El uso de matrices termoplásticas en la fabricación de materiales compuestos facilita el proceso de reciclaje de los mismos, evitando así que cuando finalicen su vida útil sean desechados en vertederos o incinerados. Es por este motivo que se plantea como un paso sucesivo, el estudio de viabilidad del reciclaje de estos perfiles de rPET mediante reciclado mecánico. Este proceso implicaría un primer paso de trituración o de molienda para reducir el tamaño del perfil y el posterior reprocesado mediante compresión, extrusión o inyección. 


\section{CONTRIBUCIONES EN CONGRESOS}

Polimerización de monómeros híbridos para la obtención de copolímeros de Bio-poliamidas de altas prestaciones. M. Asensio, M. Herrero, K. Núñez, J.C. Merino, J.M. Pastor. XIV Reunión de grupo especializado en polímeros GEP (RSEQ, RSEF). Facultad de Ciencias, Universidad de Burgos, (5-8 de septiembre de 2016).

Polimerización de monómeros híbridos para la obtención de copolímeros de Bio-poliamidas de altas prestaciones. M. Asensio, M. Herrero, K. Núñez, J.C. Merino, J.M. Pastor, III Jornada de Doctorandos del Programa de Doctorado en Ingeniería Industrial. Escuela de Ingenieros Industriales, Universidad de Valladolid (15 de septiembre de 2016).

Nanocomposites de Bio-poliamidas híbridas de altas prestaciones. $M$. Asensio, M. Herrero, K. Núñez, J.C. Merino, J.M. Pastor. IX Congreso de Jóvenes investigadores en polímeros (JIP). Universidad Rovira i Virgili, Tarragona, (5-8 de junio de 2017).

Polimerización In Situ de nanocompuestos de polipropileno isotáctico con catálisis metalocénica. M. Asensio, M. Herrero, K. Núñez, J.C. Merino, J.M. Pastor. XII Congreso Nacional de materiales compuestos (MATCOMP). Donostia/San Sebastian, (21-23 de junio de 2017).

Bio-nanocomposites de poliamida 4.10 y 6.10: Estudio del procesado y propiedades termo-mecánicas. M. Asensio, M. Herrero, K. Núñez, J.C. Merino, J.M. Pastor. IV Jornada de Doctorandos del Programa de Doctorado en Ingeniería Industrial. Escuela de Ingenieros Industriales, Universidad de Valladolid, (14 de septiembre de 2017).

Effects of fiber orientation on the thermo-mechanical properties of sepiolite reinforced bio-polyamide. M. Asensio, M. Herrero, L.E Alonso, K. Núñez, J.M. Pastor, XV Congreso Nacional de materiales (CNMAT). Salamanca (46 de Julio de 2018).

Rheological characterization of polyethylene(terephthalate) modified by reactive extrusion. M. Asensio, J. Guerrero, K. Núñez, M. Herrero, JM. Pastor, XV Reunión del grupo especializado de polímeros (GEP) de la RSEQ YRSEF. Huelva (2427 de septiembre de 2018). 
Rheological modification of PET waste. Thermoplastic pultrusion as recycling technology. M. Asensio, P. Esfandiari, K. Núñez, J. Guerrero, M. Herrero, J.C. Merino, J.M. Pastor, Congreso Nacional de materiales compuestos (MATCOMP), Vigo (3-5 de julio de 2019).

\section{PUBLICACIONES DE LA TESIS DOCTORAL}

Rheological modification of recycled poly(ethylene terephthalate): blending and reactive extrusion. M. Asensio, K. Núñez, J. Guerrero, M. Herrero, J.C. Merino, J.M. Pastor, Polymer Degradation and stability, Volume 179, 2020. doi:10.1016/j.polymdegradstab.2020.109258.

Processing of pre-impregnated thermoplastic towpreg reinforced by continuous glass fibre and recycled PET by pultrusion. $M$. Asensio, $P$. Esfandiari, K. Núñez, J. F. Silva, A. Marques, J.C Merino, J.M. Pastor, Comosites Part B: Engineering, JCOMB_2020_2535, under decision.

\section{PUBLICACIONES RELACIONADAS CON LA TESIS DOCTORAL}

In situ polymerization of isotactic polypropylene sepiolite nanocomposites and its copolymers by metallocene catalysis. M. Asensio. M. Herrero, K. Núñez, R. Gallego, J. C. Merino, J. M. Pastor, European Polymer Journal, Volume 100, Pages 278-289, 2018. doi:10.1016/j.eurpolymj.2018.01.034.

Morphological, Thermal, and Mechanical Behavior of Polyamide11/Sepiolite Bio-Nanocomposites Prepared by Melt Compounding and In Situ Polymerization. M. Herrero, M. Asensio, K. Núñez, J.C. Merino, J. M. Pastor, Polymer Composites, Volume 40, Issue S1, Pages E704E713, 2018. doi:10.1002/pc.24962.

The Influence of Sepiolite Orientation and Concentration, on the Morphological, Thermal and Mechanical Properties of Bio-Polyamide 4.10 Nanocomposites. M. Asensio, M. Herrero K. Núñez, J.C. Merino, J. M. Pastor, Polymer engineering and science, Volume 60, Issue 5, Pages 1035-1043, 2020. doi:10.1002/pen.25359.

Control of branches distribution in linear PE copolymers using fibrillar nanoclay as support of catalyst system. P. Tanasi, M. Asensio, M. Herrero, K. Núñez, E. Cañibano, J.C. Merino, Polymer, 2020, POLYMER-19-3006. 